\title{
Strategies toward the total synthesis of calyciphylline B-type alkaloids: A computational perspective aided by DFT analysis
}

Amit Kumar Chattopadhyay, ${ }^{\dagger}$ Gilles Berger, ${ }^{\ddagger}$ and Stephen Hanessian ${ }^{\dagger}{ }^{*}$

${ }^{\dagger}$ Department of Chemistry, Université de Montréal, Station Centre Ville, C.P. 6128, Montreal, Qc, H3C 3J7, Canada.

${ }^{\ddagger}$ Laboratoire de Chimie Pharmaceutique Organique, Campus Plaine CP205/5, Université Libre de Bruxelles, Bd du Triomphe, 1050 Brussels, Belgium.

Email: stephen.hanessian@umontreal.ca

Table of content
1. Copies of ${ }^{1} \mathrm{H}$ NMR and ${ }^{13} \mathrm{C}$ NMR spectra
S2-S61
2. Copies of 2D spectra
S62-S68
3. DFT data (computational details)
S69-S129
4. X-ray data
S130-S140
5. References
S141 


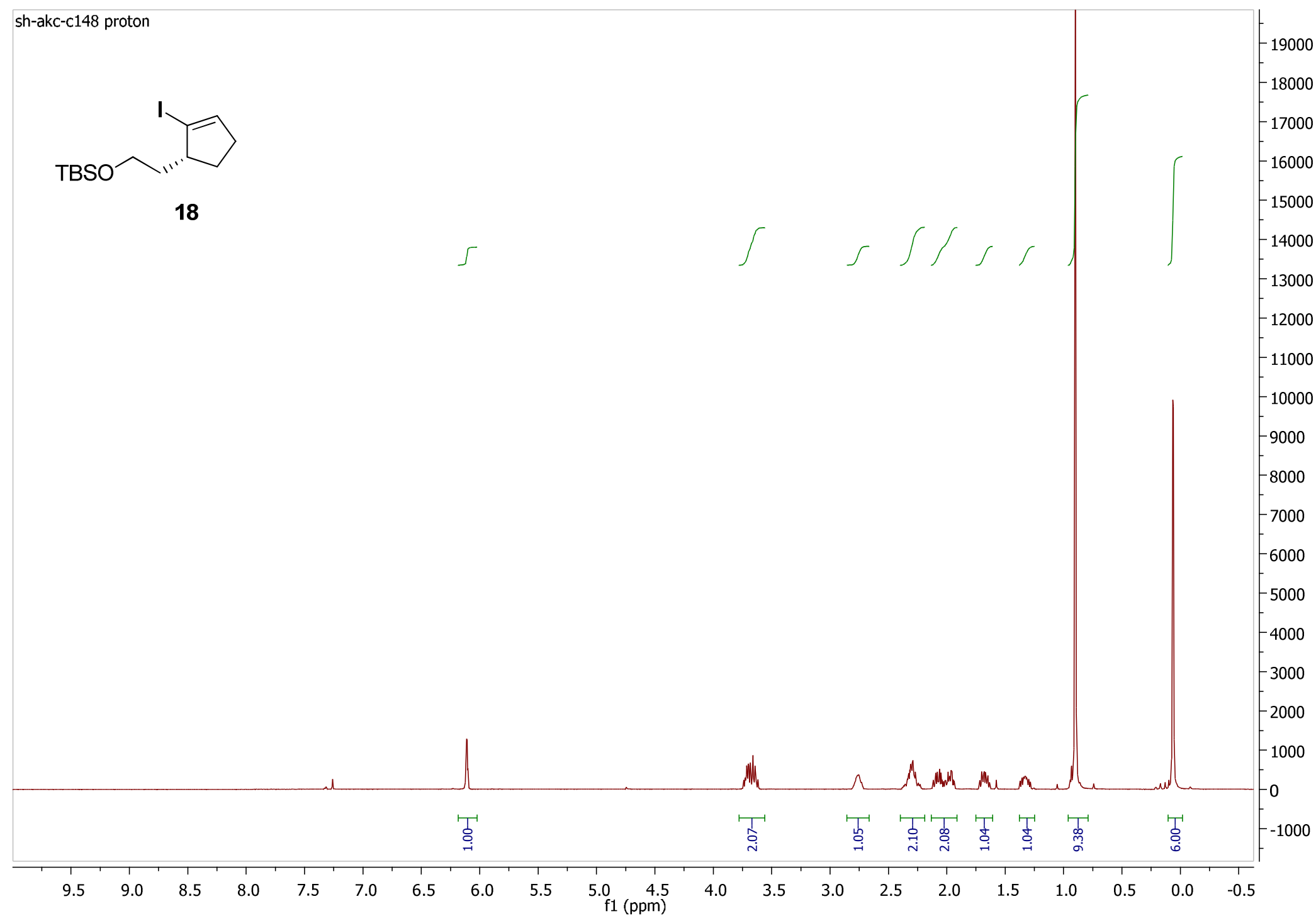

18, ( ${ }^{1} \mathrm{H}$ NMR, $\left.\mathrm{CDCl}_{3}, 400 \mathrm{MHz}\right)$ 


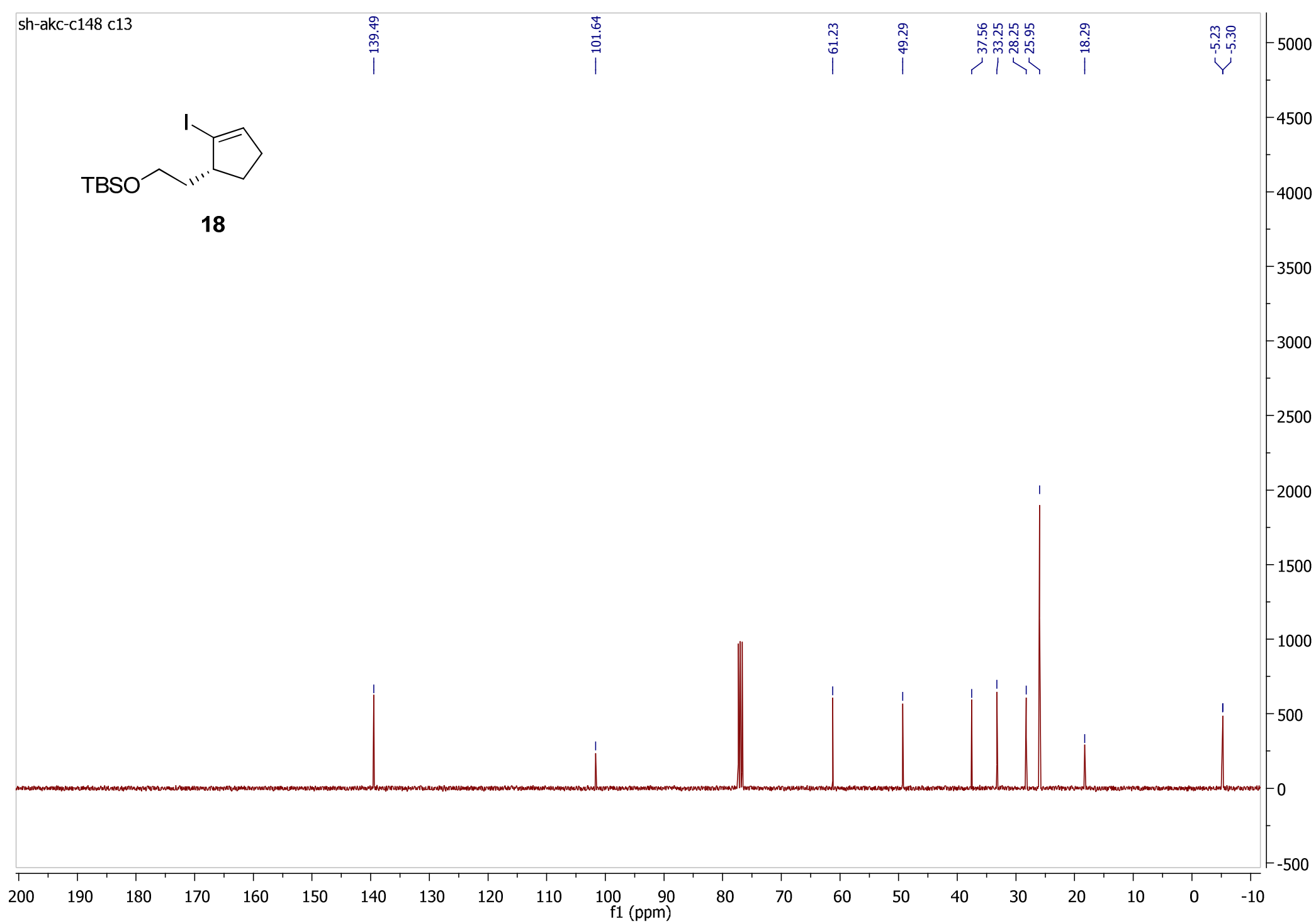

18, $\left({ }^{13} \mathrm{C} \mathrm{NMR}, \mathrm{CDCl}_{3}, 100 \mathrm{MHz}\right)$ 


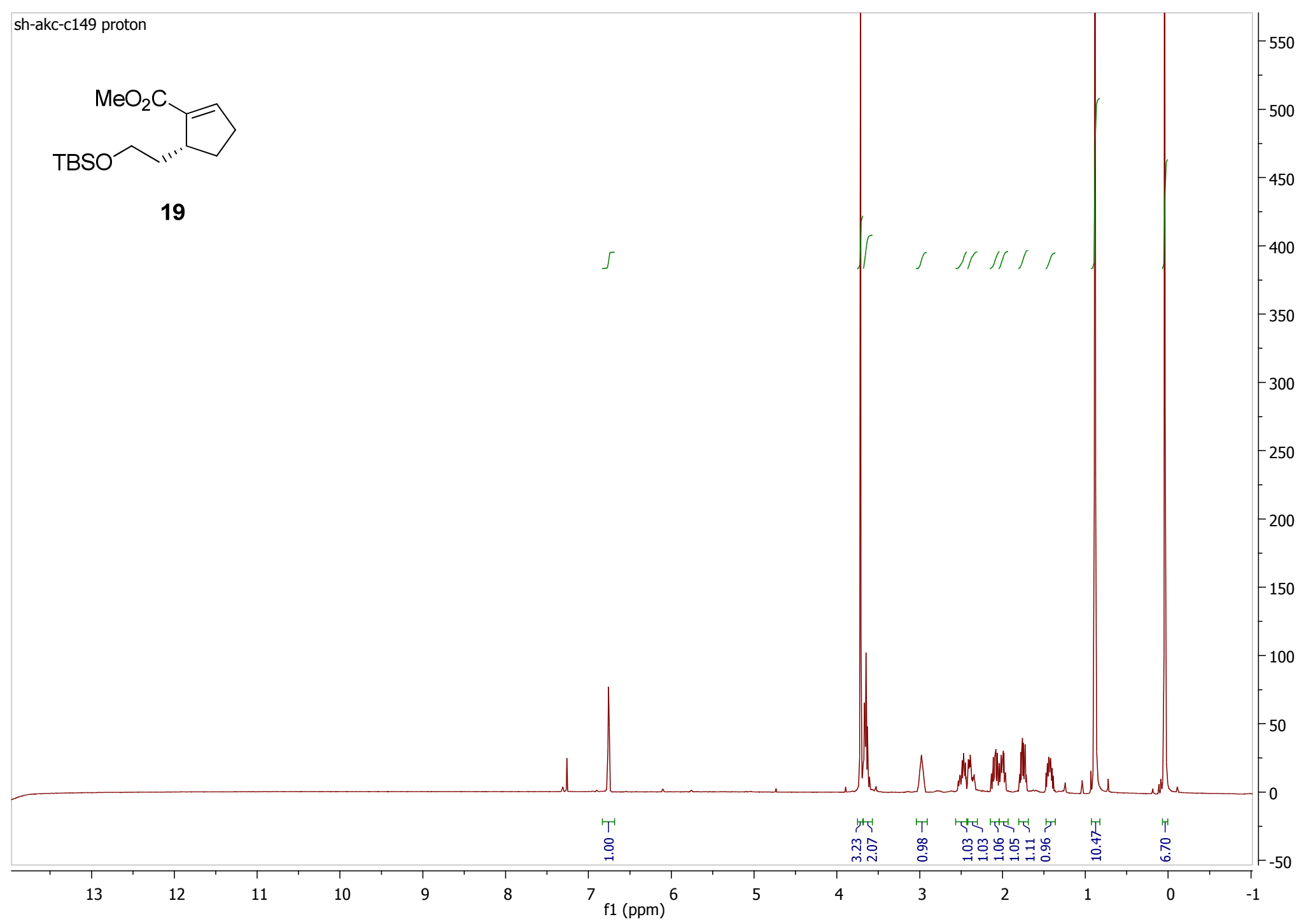

19, ( $\left.{ }^{1} \mathrm{H} \mathrm{NMR}, \mathrm{CDCl}_{3}, 400 \mathrm{MHz}\right)$ 


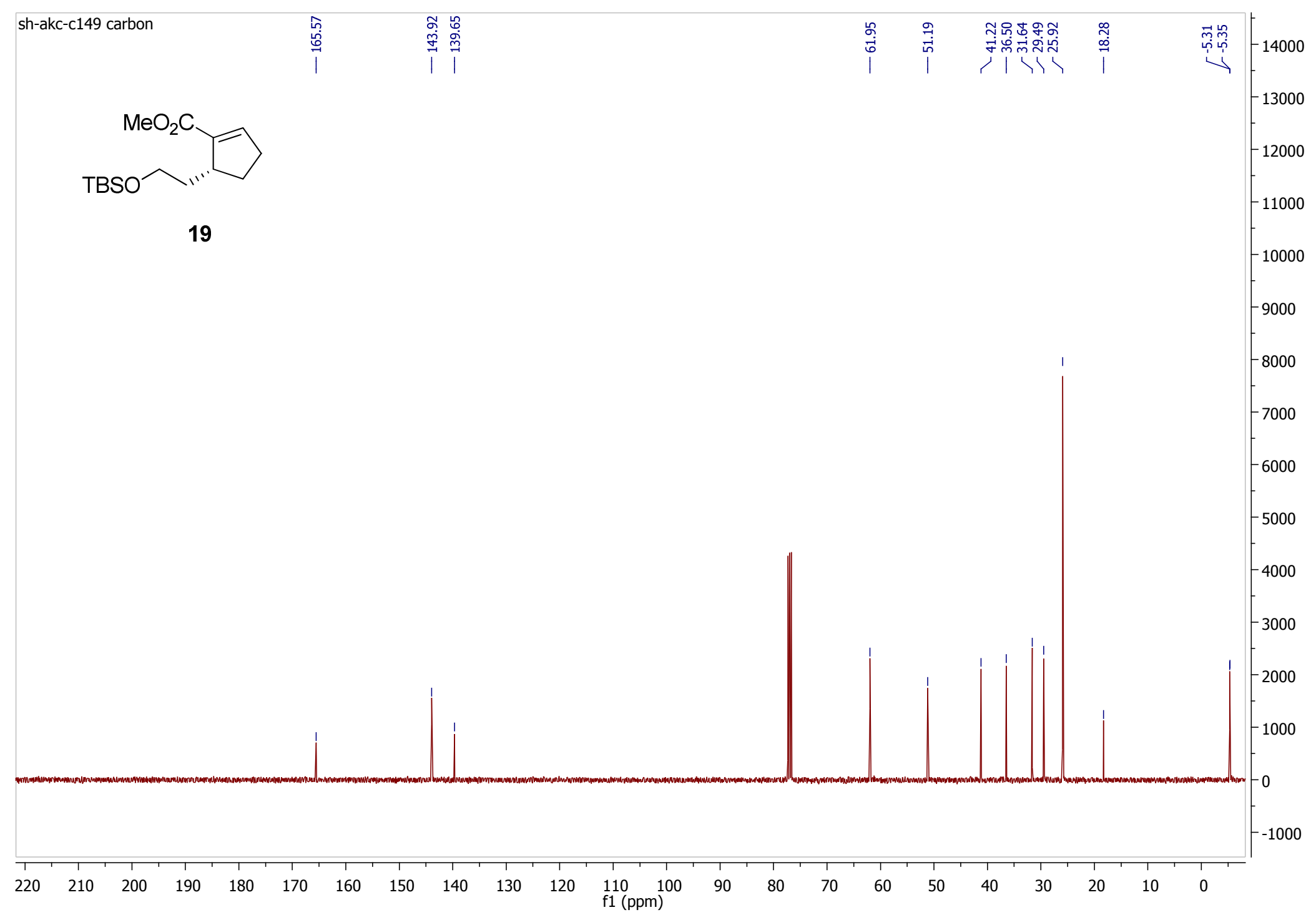

19, $\left({ }^{13} \mathrm{C} \mathrm{NMR}, \mathrm{CDCl}_{3}, 100 \mathrm{MHz}\right)$ 


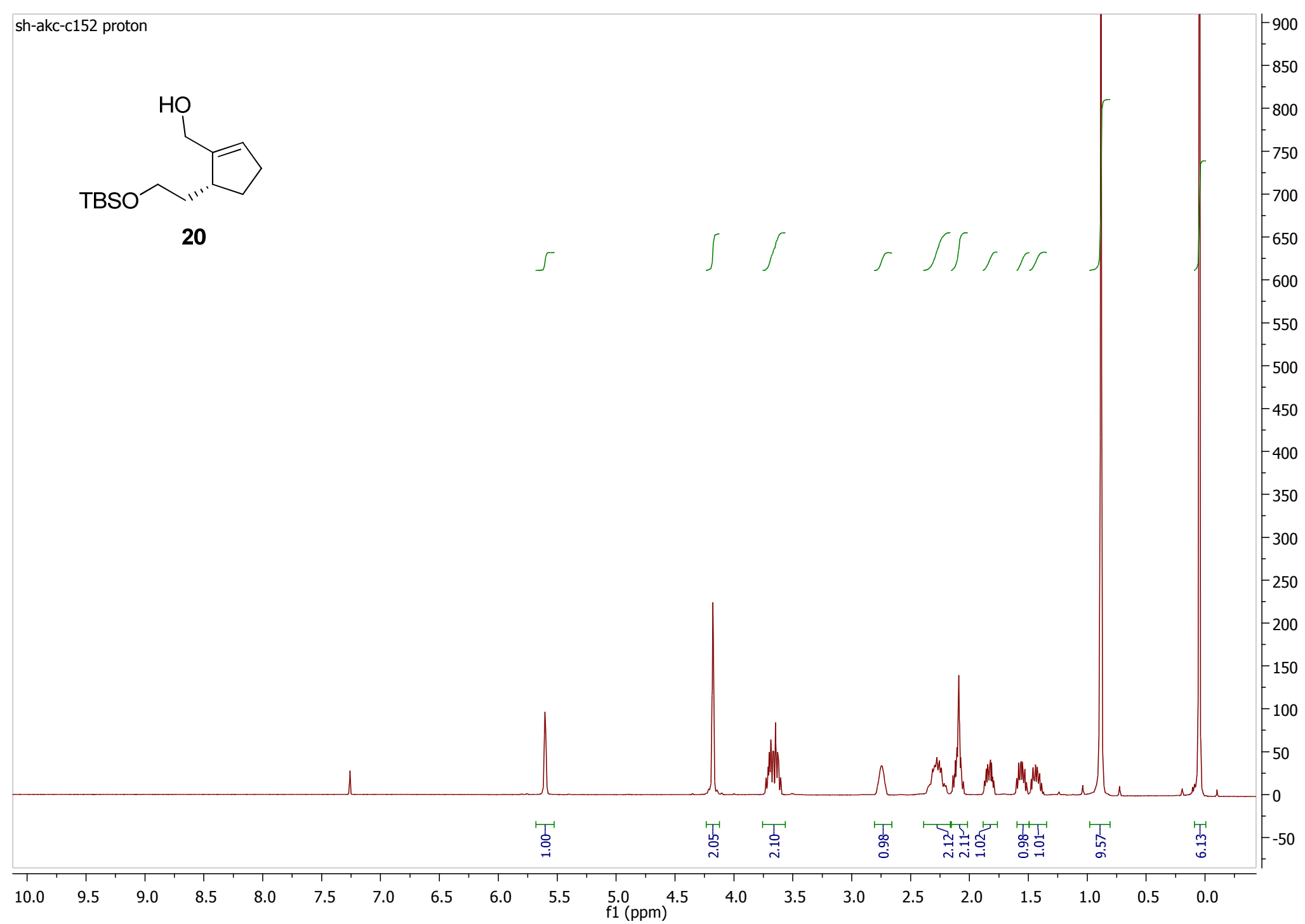

20, $\left({ }^{1} \mathrm{H} N M R, \mathrm{CDCl}_{3}, 400 \mathrm{MHz}\right)$ 


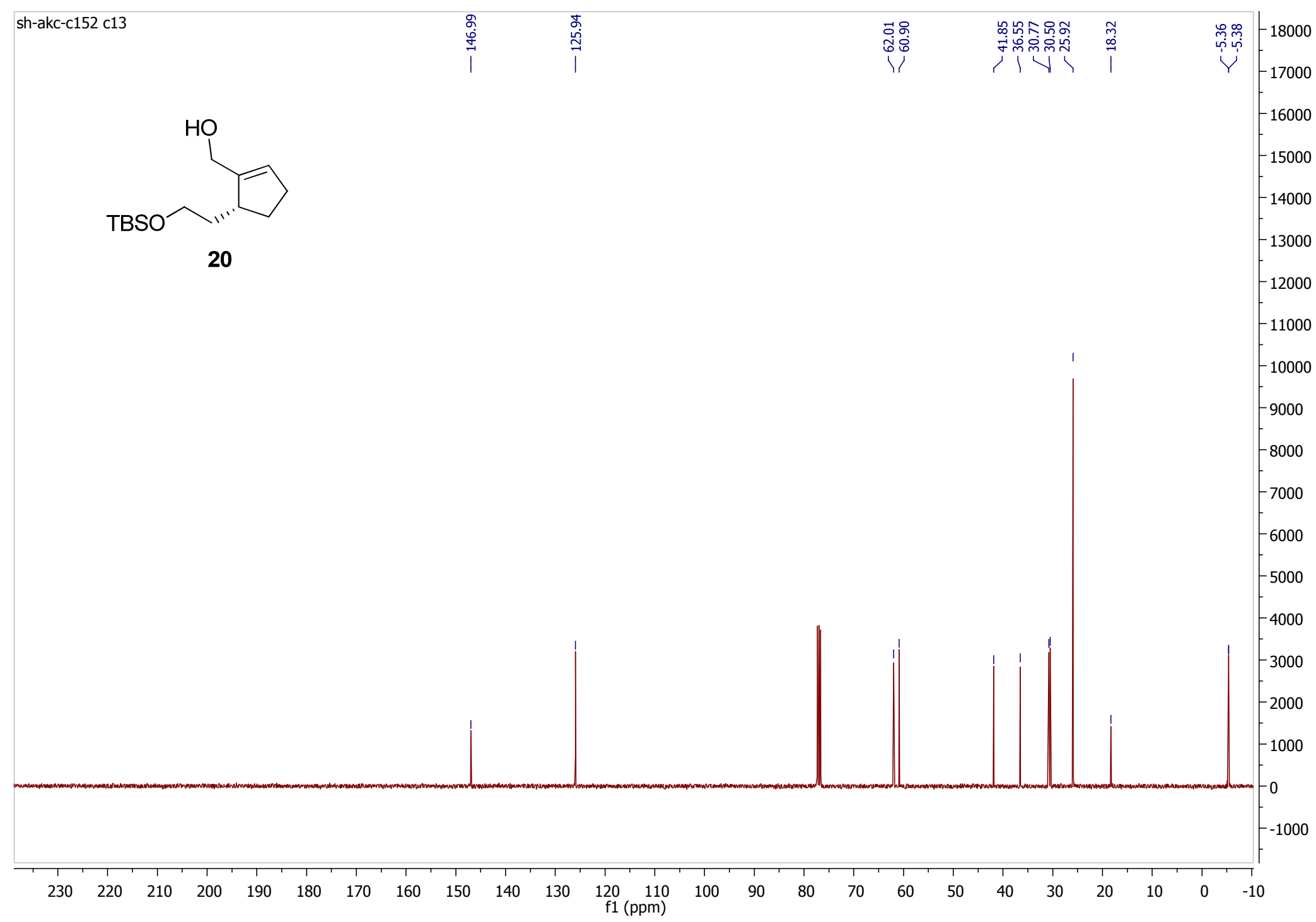

20, $\left({ }^{13} \mathrm{C} \mathrm{NMR}, \mathrm{CDCl}_{3}, 100 \mathrm{MHz}\right)$ 


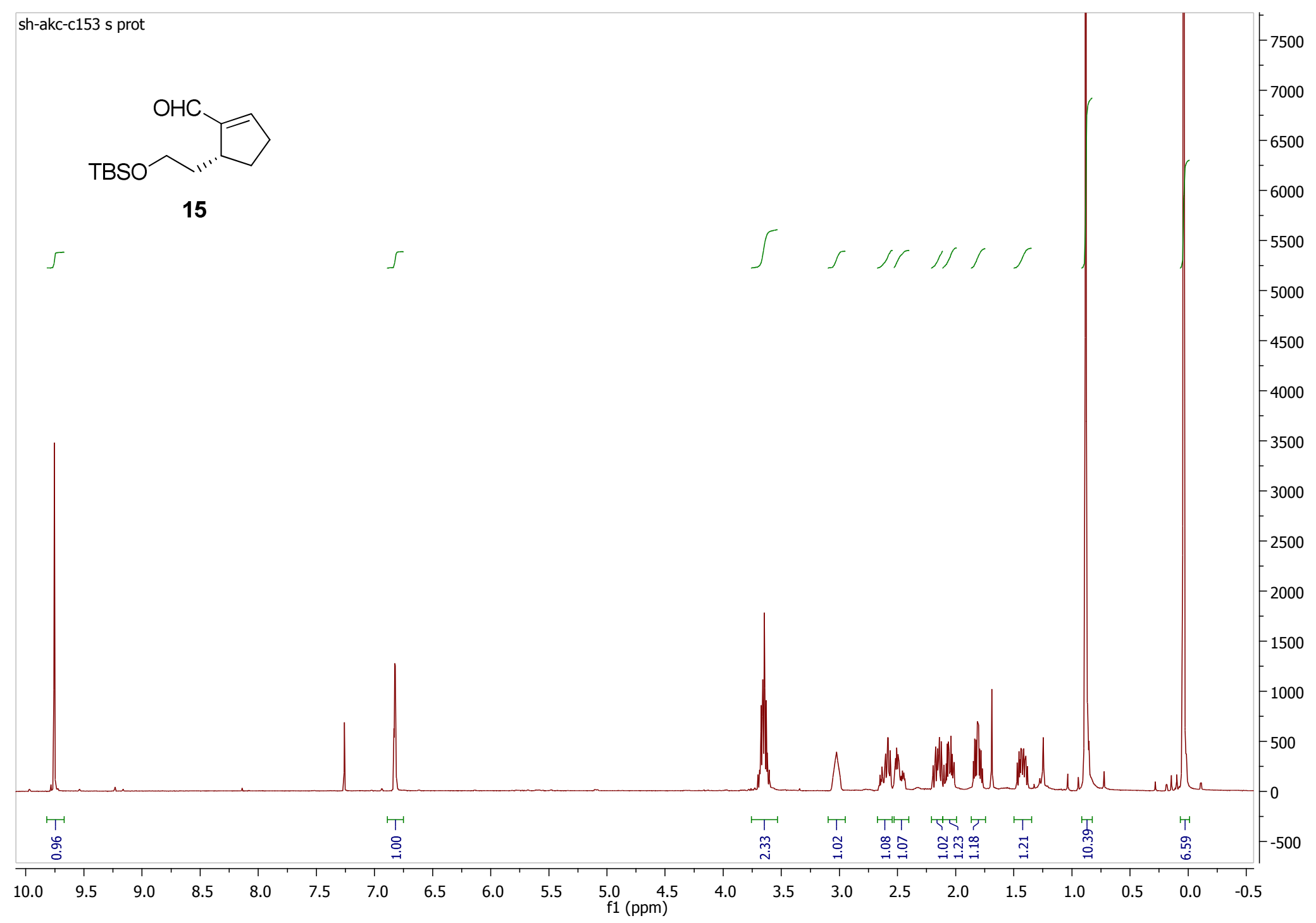

15, ( ${ }^{1} \mathrm{H}$ NMR, $\left.\mathrm{CDCl}_{3}, 400 \mathrm{MHz}\right)$ 


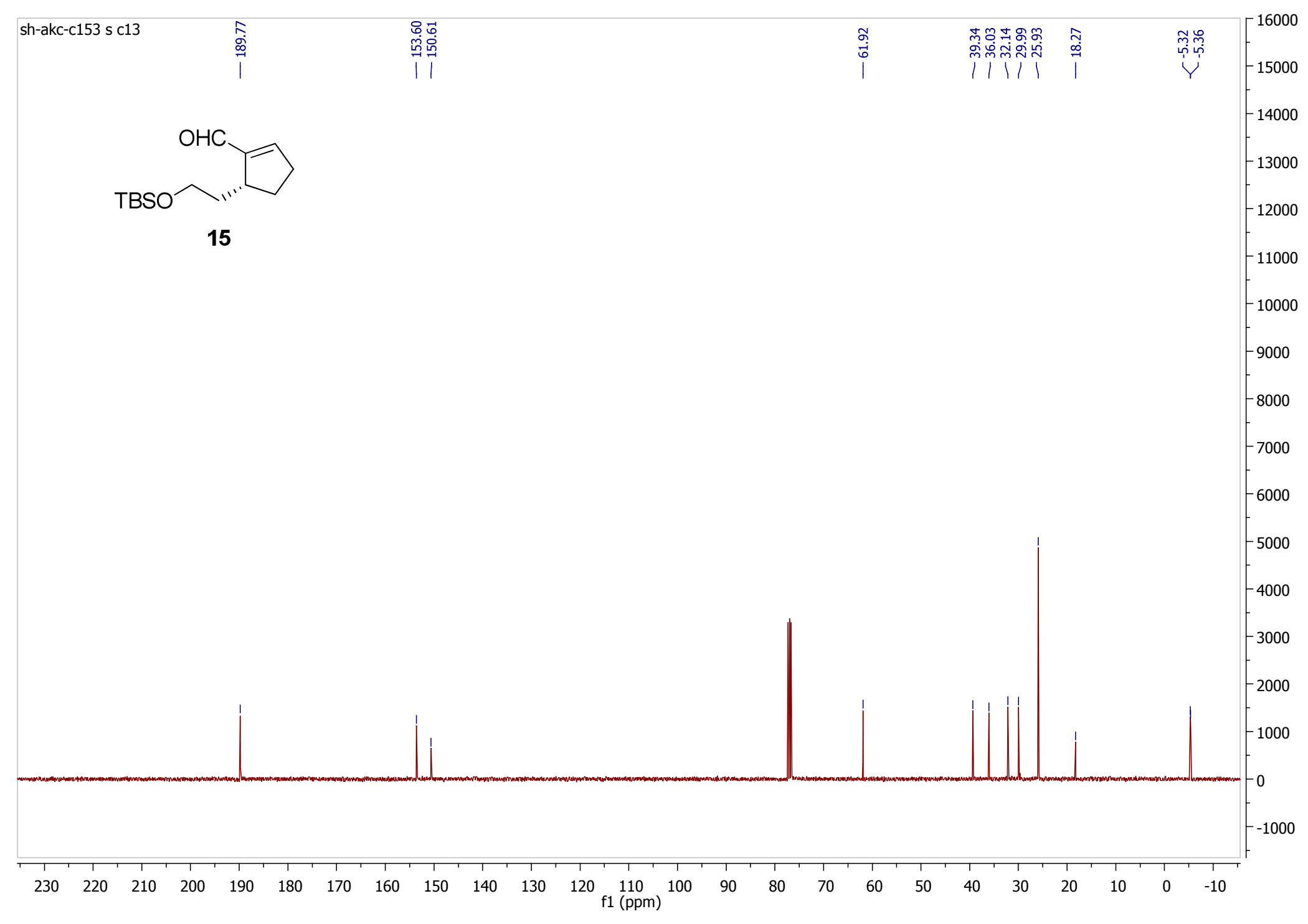

15, $\left({ }^{13} \mathrm{C} \mathrm{NMR}, \mathrm{CDCl}_{3}, 100 \mathrm{MHz}\right)$ 


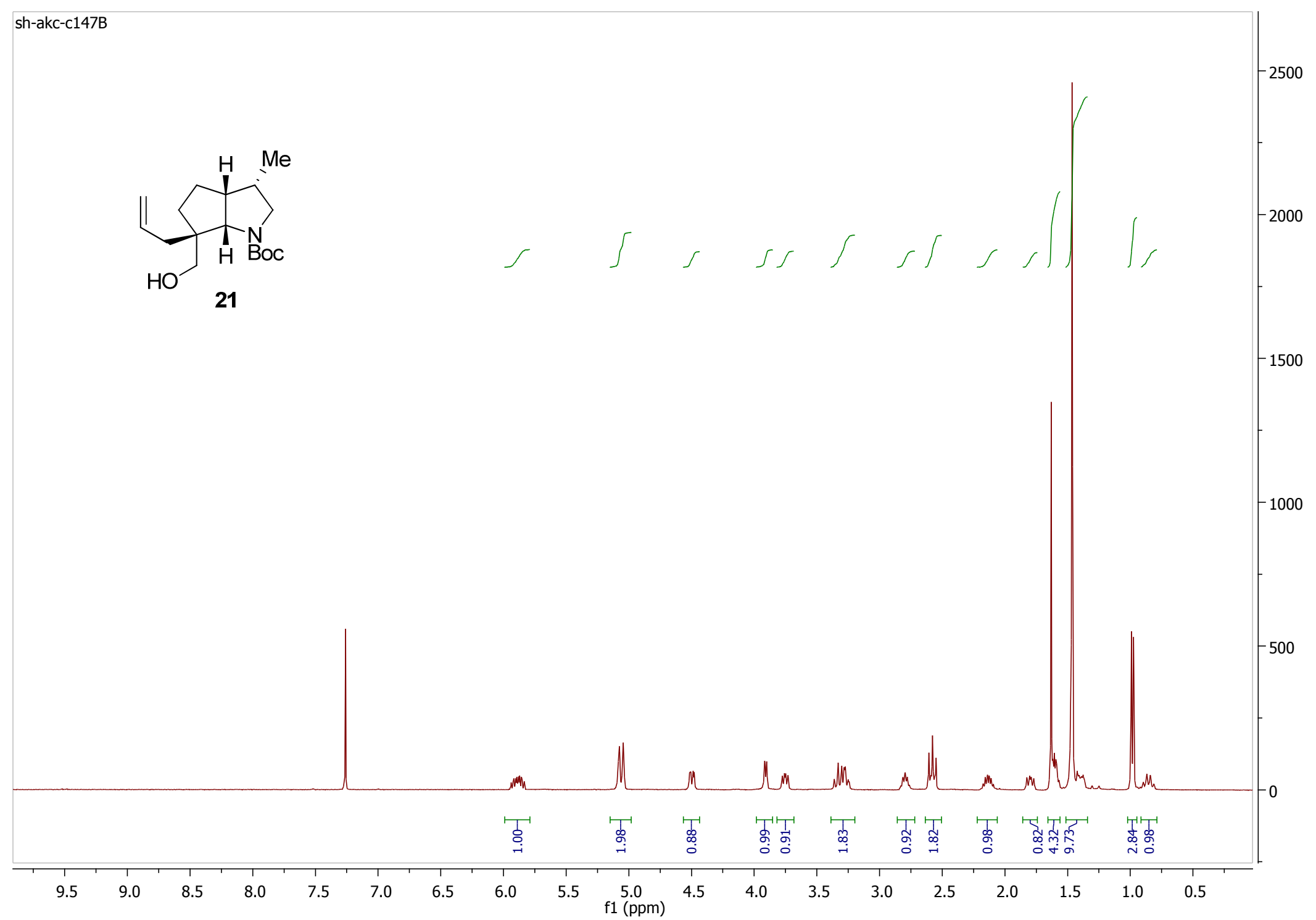

21, $\left({ }^{1} \mathrm{H} N M R, \mathrm{CDCl}_{3}, 400 \mathrm{MHz}\right)$ 


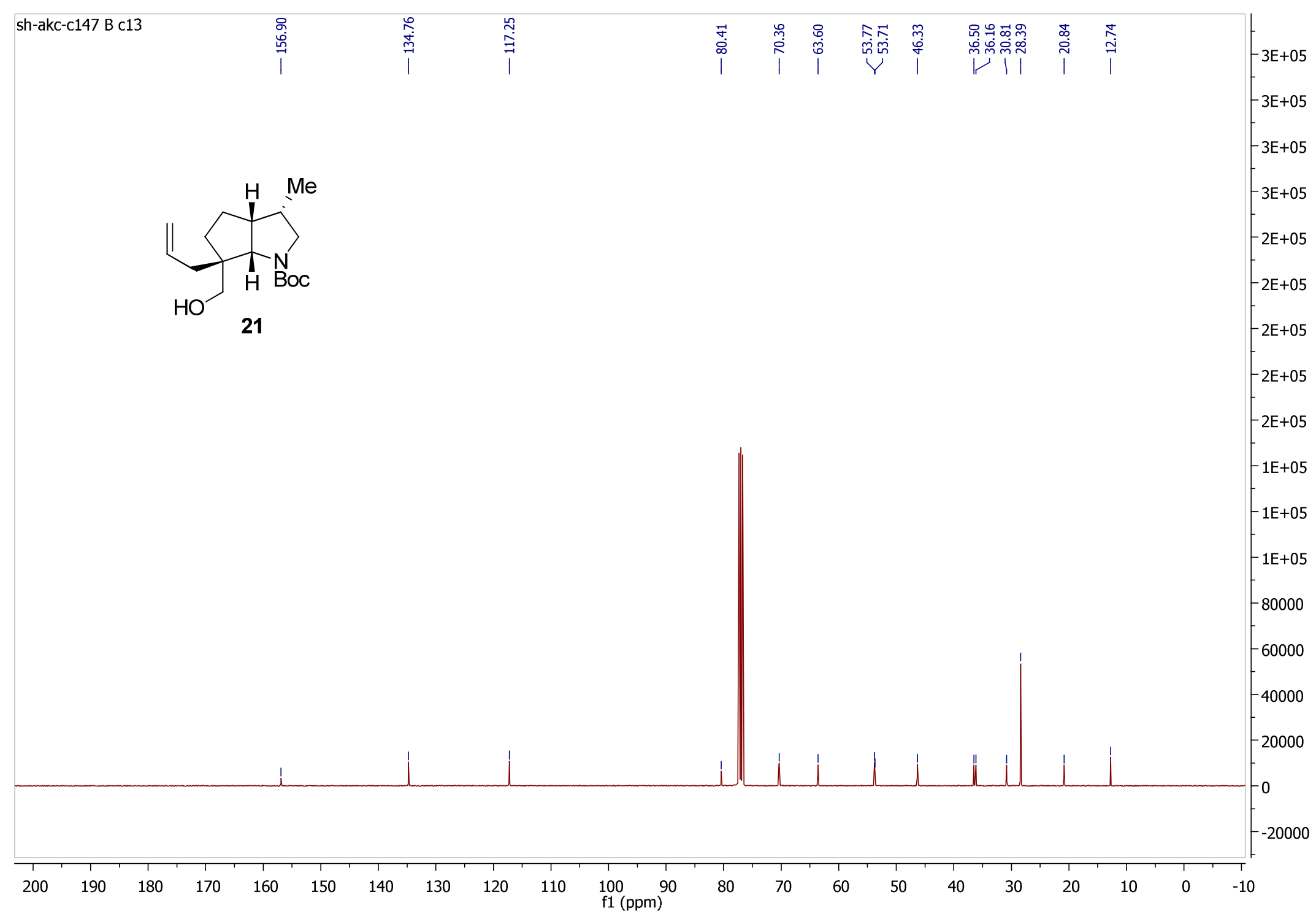

21, $\left({ }^{13} \mathrm{C} \mathrm{NMR}, \mathrm{CDCl}_{3}, 100 \mathrm{MHz}\right)$ 


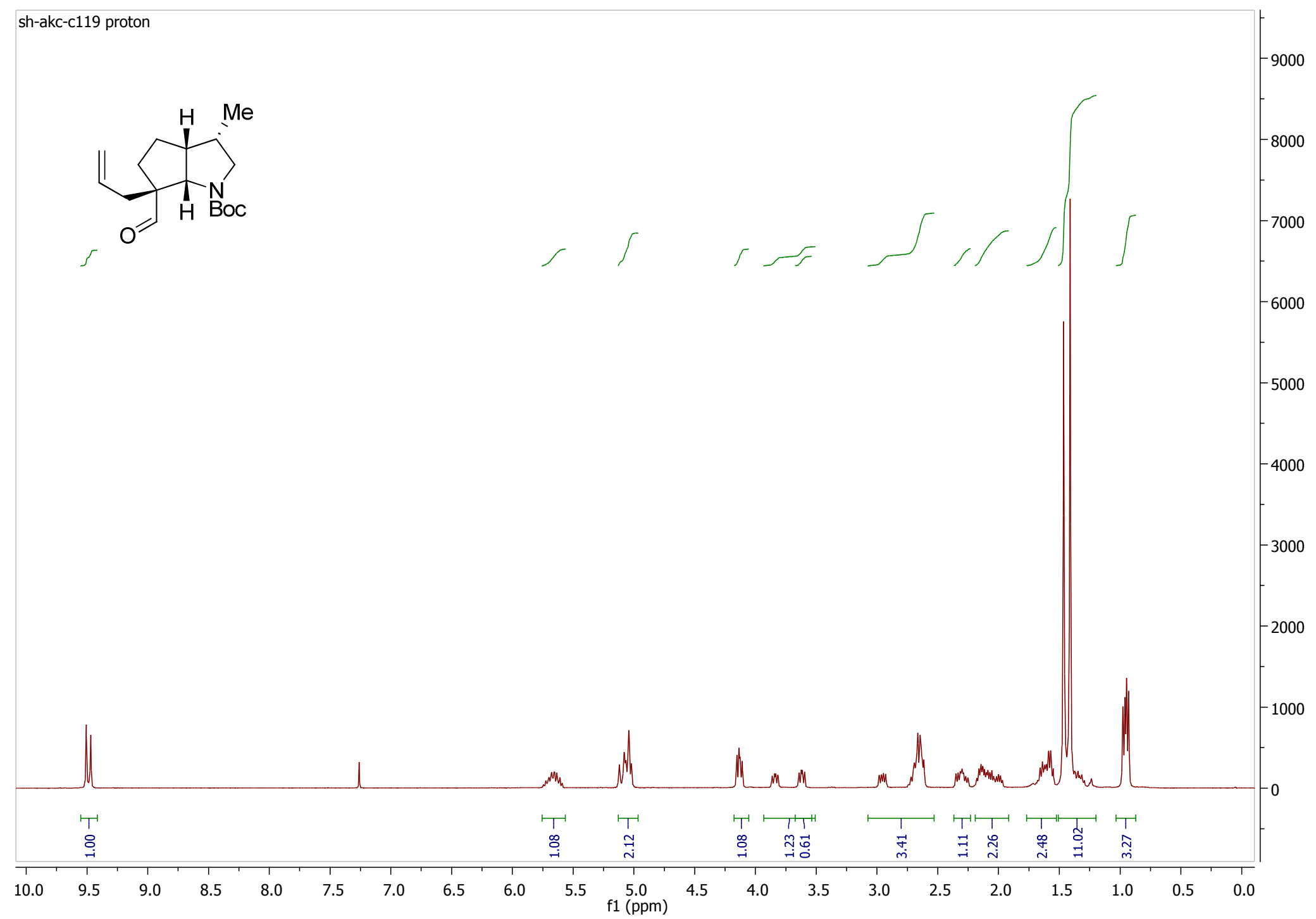

$\left({ }^{1} \mathrm{H} \mathrm{NMR}, \mathrm{CDCl}_{3}, 400 \mathrm{MHz}\right)$ 


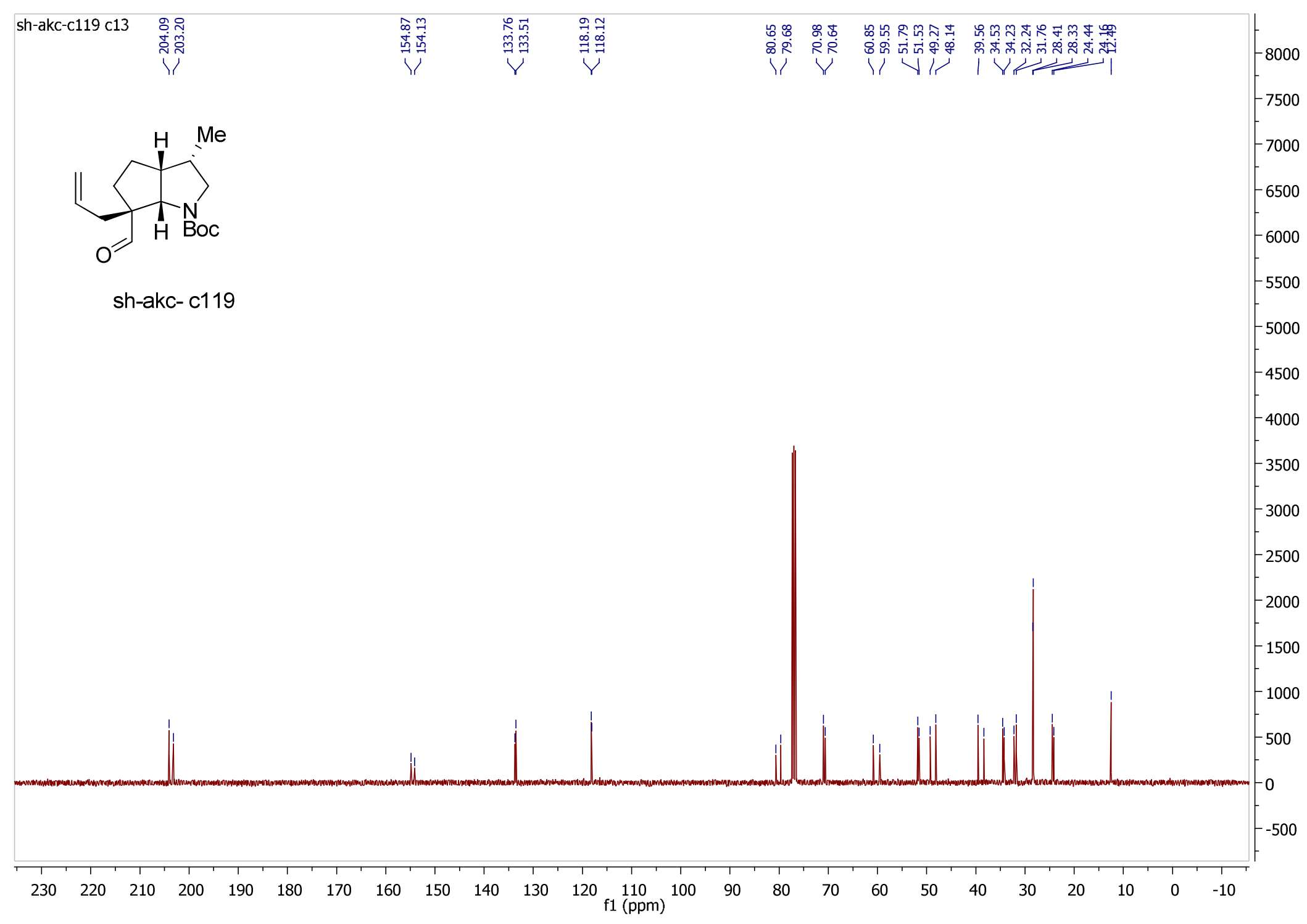

$\left({ }^{13} \mathrm{CNMR} \mathrm{CDCl}_{3}, 100 \mathrm{MHz}\right)$ 


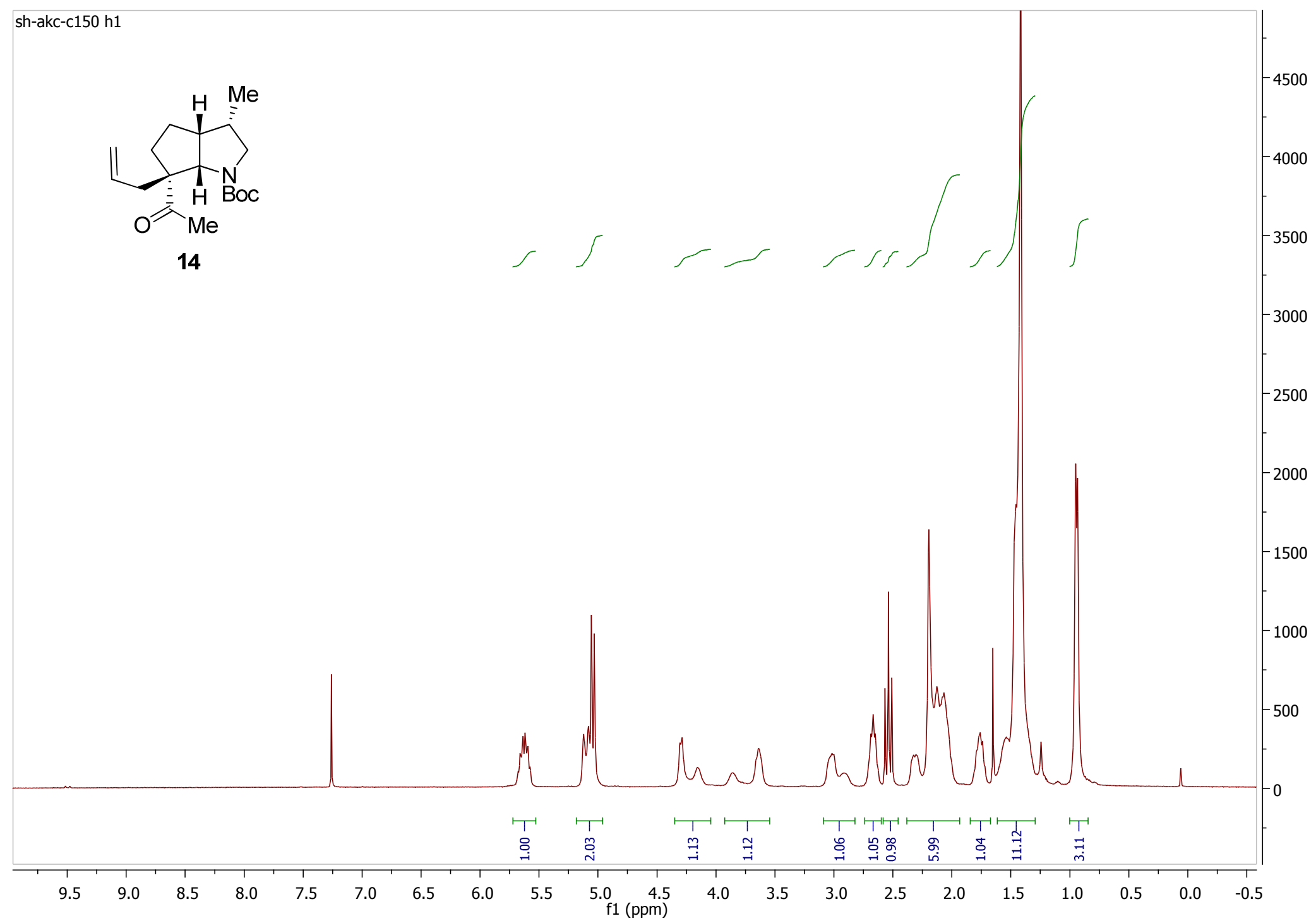

14, $\left({ }^{1} \mathrm{H} \mathrm{NMR}, \mathrm{CDCl}_{3}, 400 \mathrm{MHz}\right)$ 


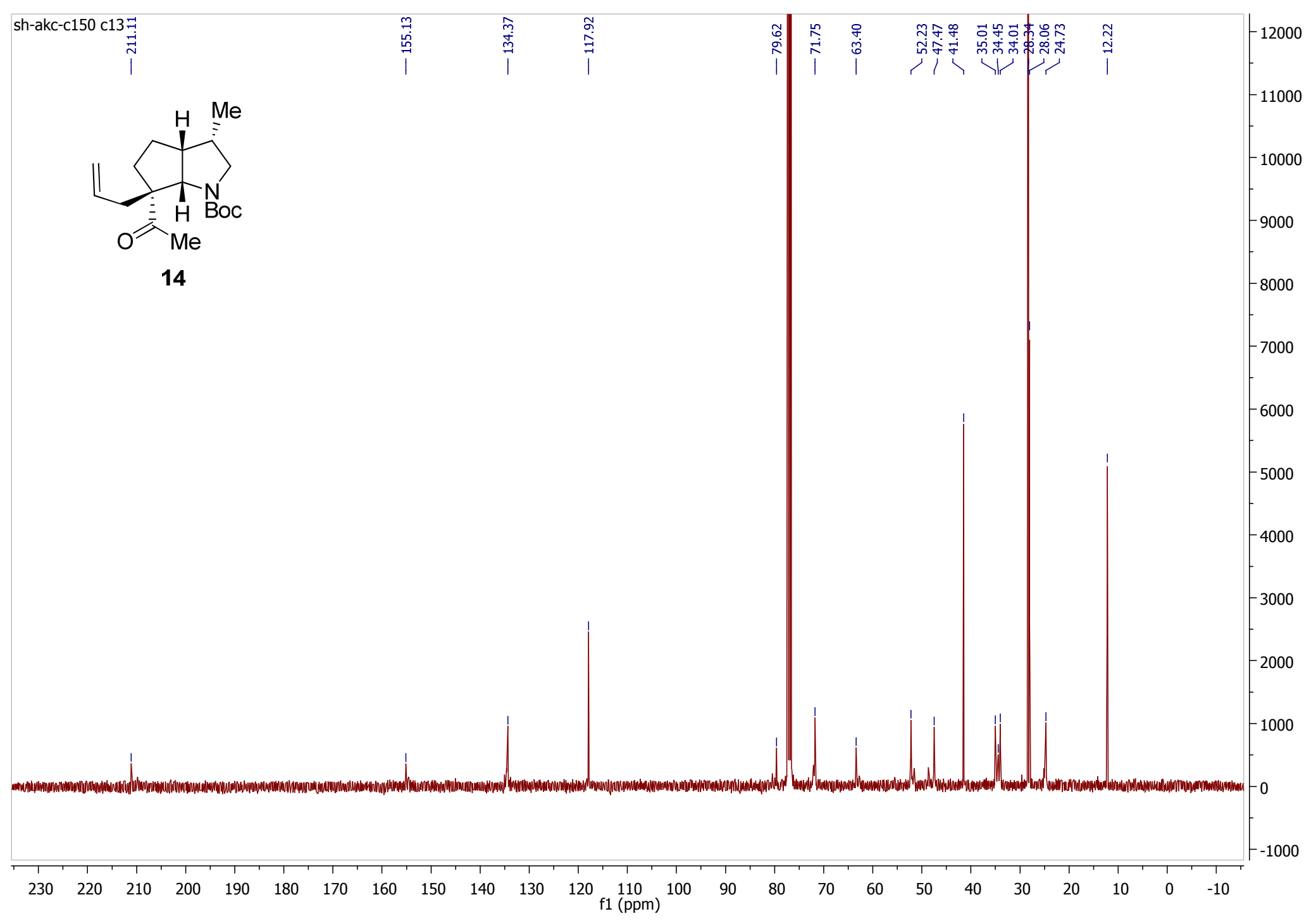

14, $\left({ }^{13} \mathrm{C} \mathrm{NMR}, \mathrm{CDCl}_{3}, 100 \mathrm{MHz}\right)$ 


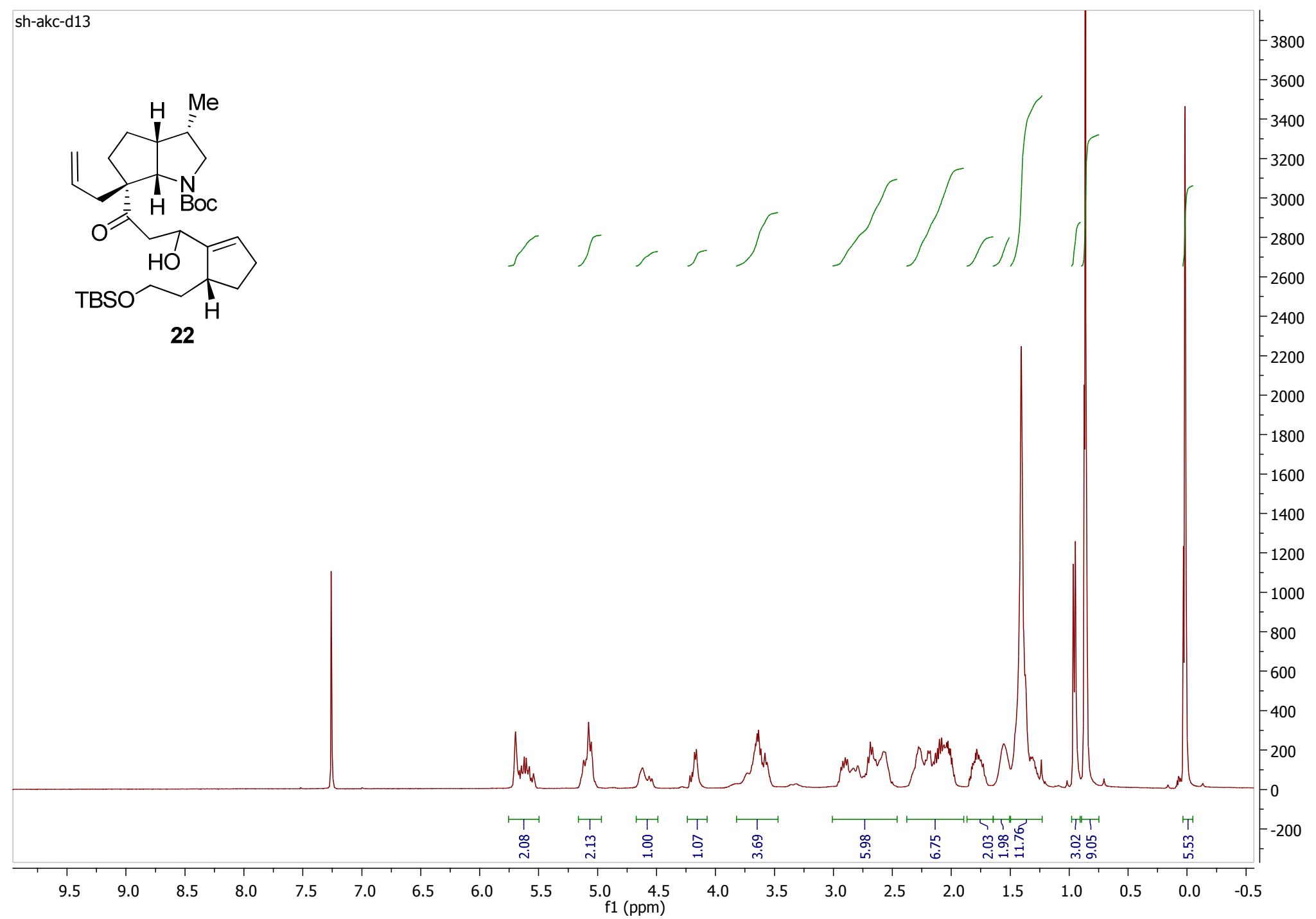

22, $\left({ }^{1} \mathrm{H} N M R, \mathrm{CDCl}_{3}, 400 \mathrm{MHz}\right)$ 


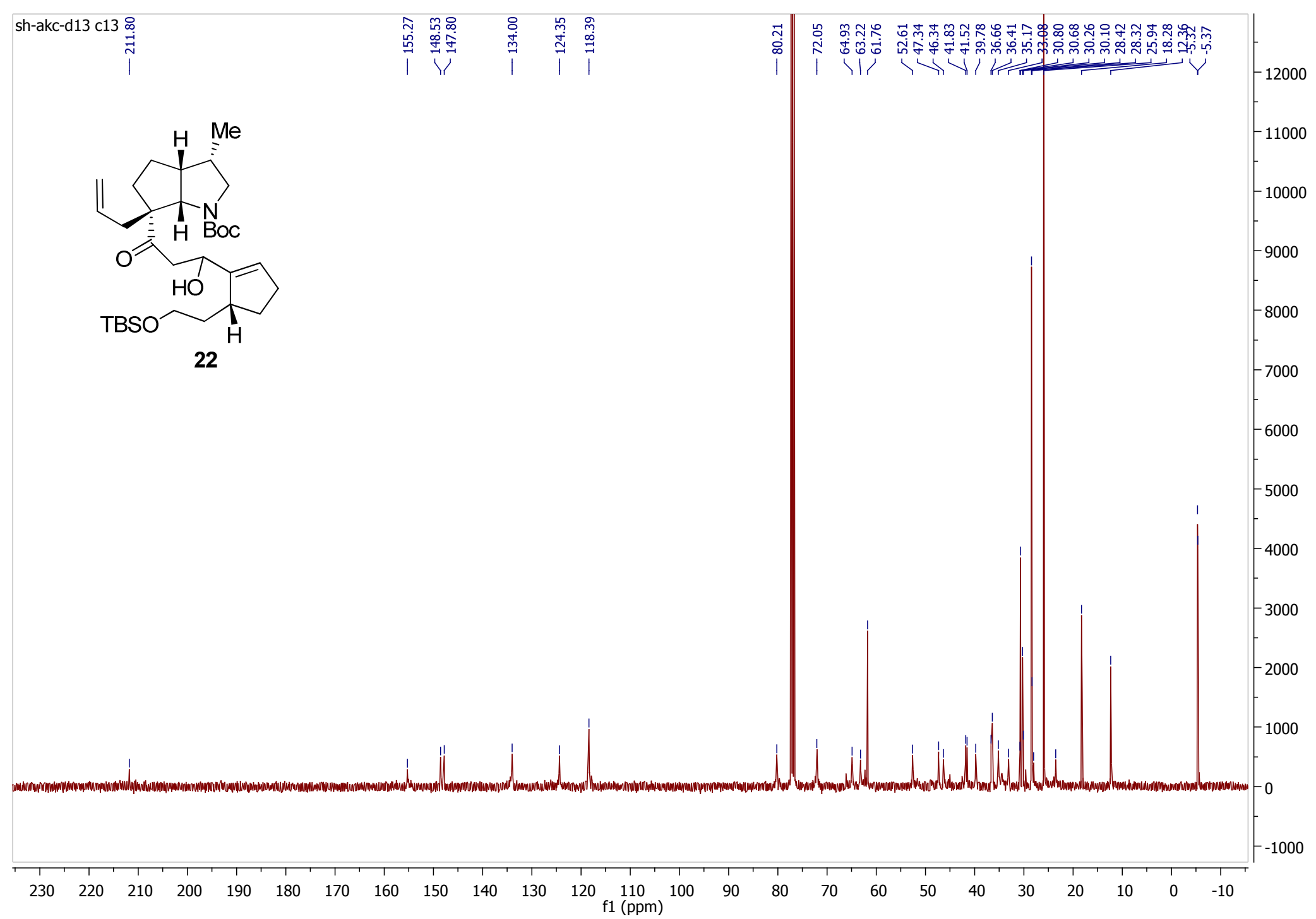

22, $\left({ }^{13} \mathrm{C} \mathrm{NMR}, \mathrm{CDCl}_{3}, 100 \mathrm{MHz}\right)$ 


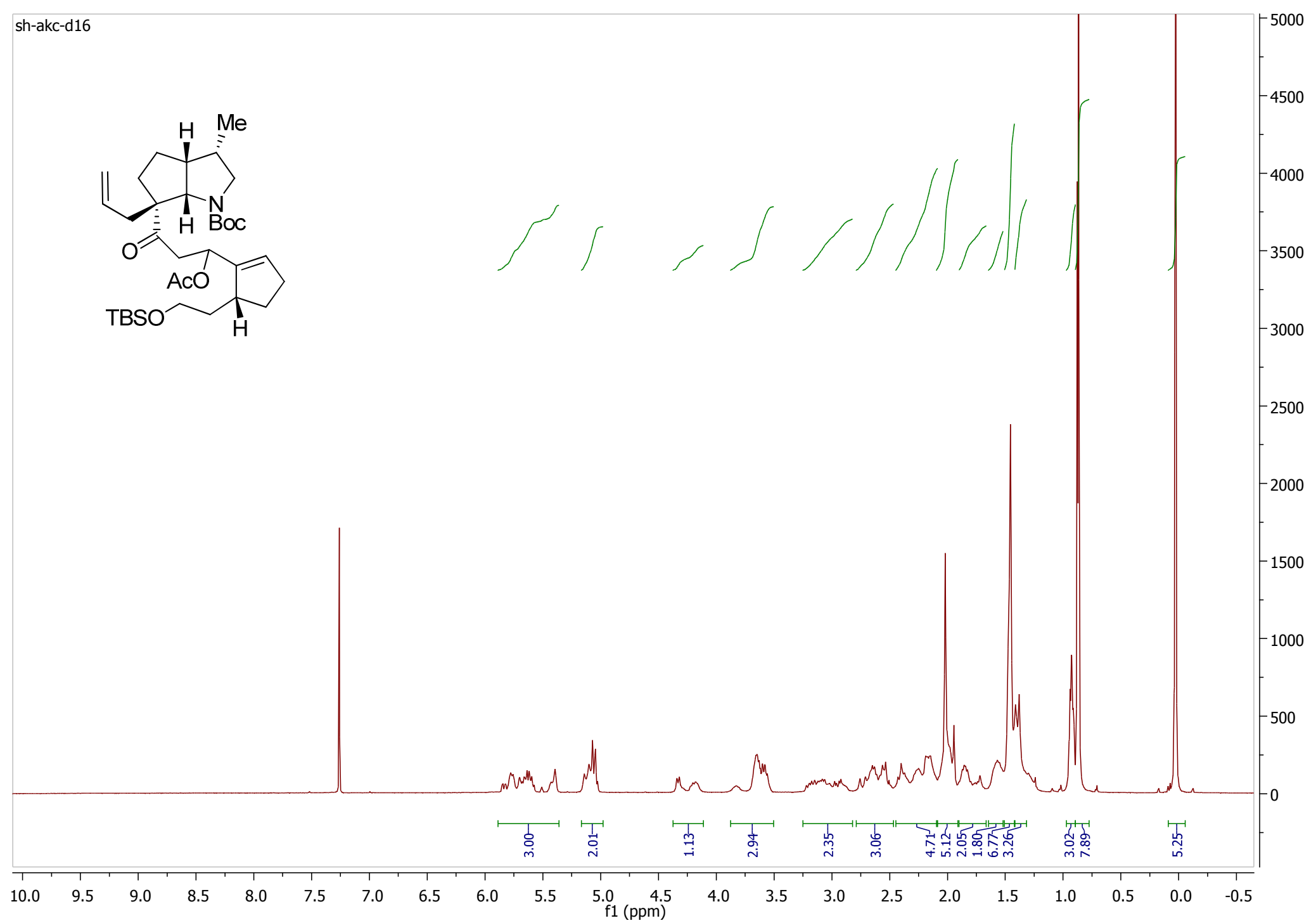

$\left({ }^{1} \mathrm{H} \mathrm{NMR}, \mathrm{CDCl}_{3}, 400 \mathrm{MHz}\right)$ 


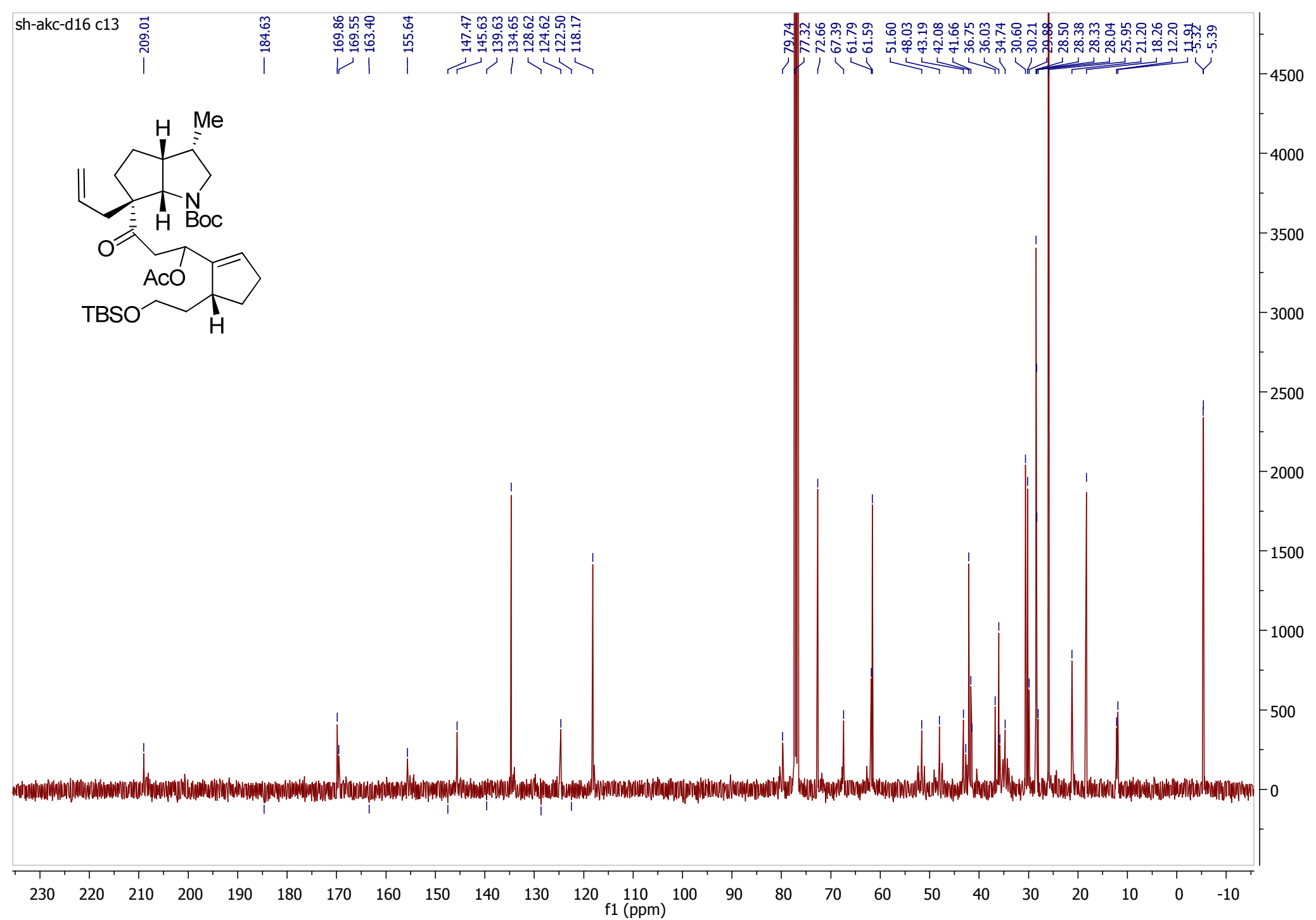

$\left({ }^{13} \mathrm{C} \mathrm{NMR}, \mathrm{CDCl}_{3}, 100 \mathrm{MHz}\right)$ 
sh-akc-d15
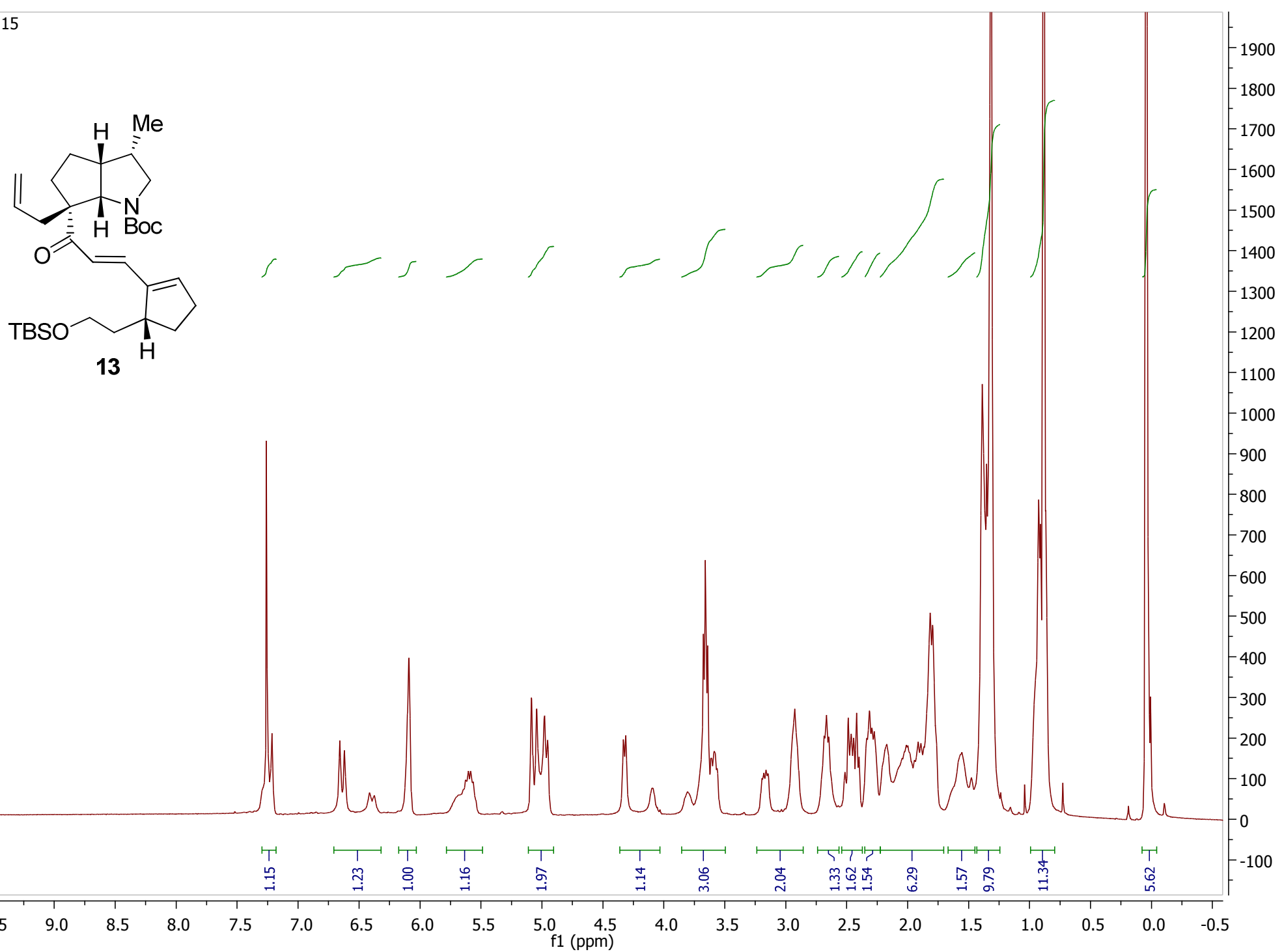

13, $\left({ }^{1} \mathrm{H} \mathrm{NMR}, \mathrm{CDCl}_{3}, 400 \mathrm{MHz}\right)$ 


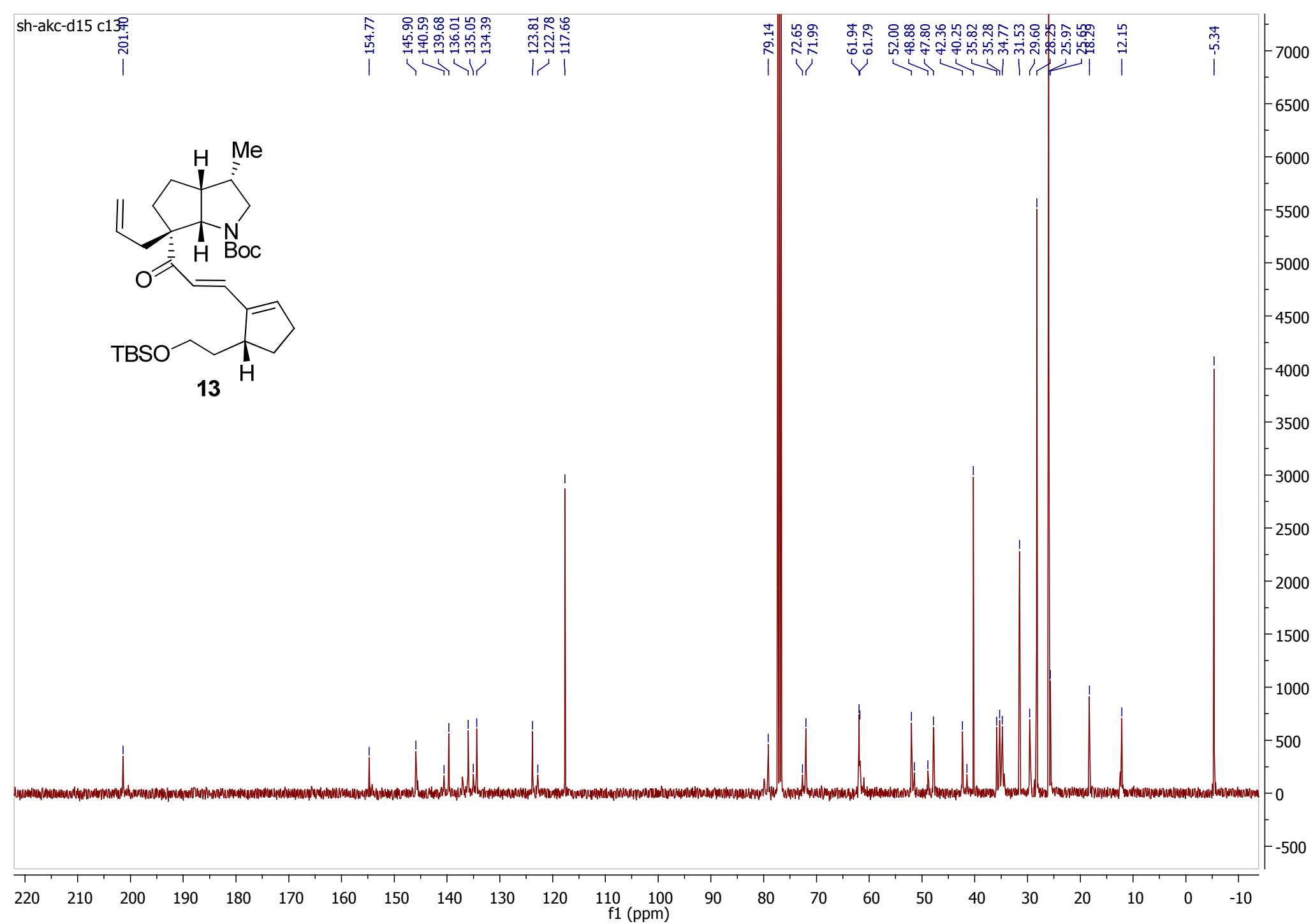

13, $\left({ }^{13} \mathrm{C} \mathrm{NMR}, \mathrm{CDCl}_{3}, 100 \mathrm{MHz}\right)$ 


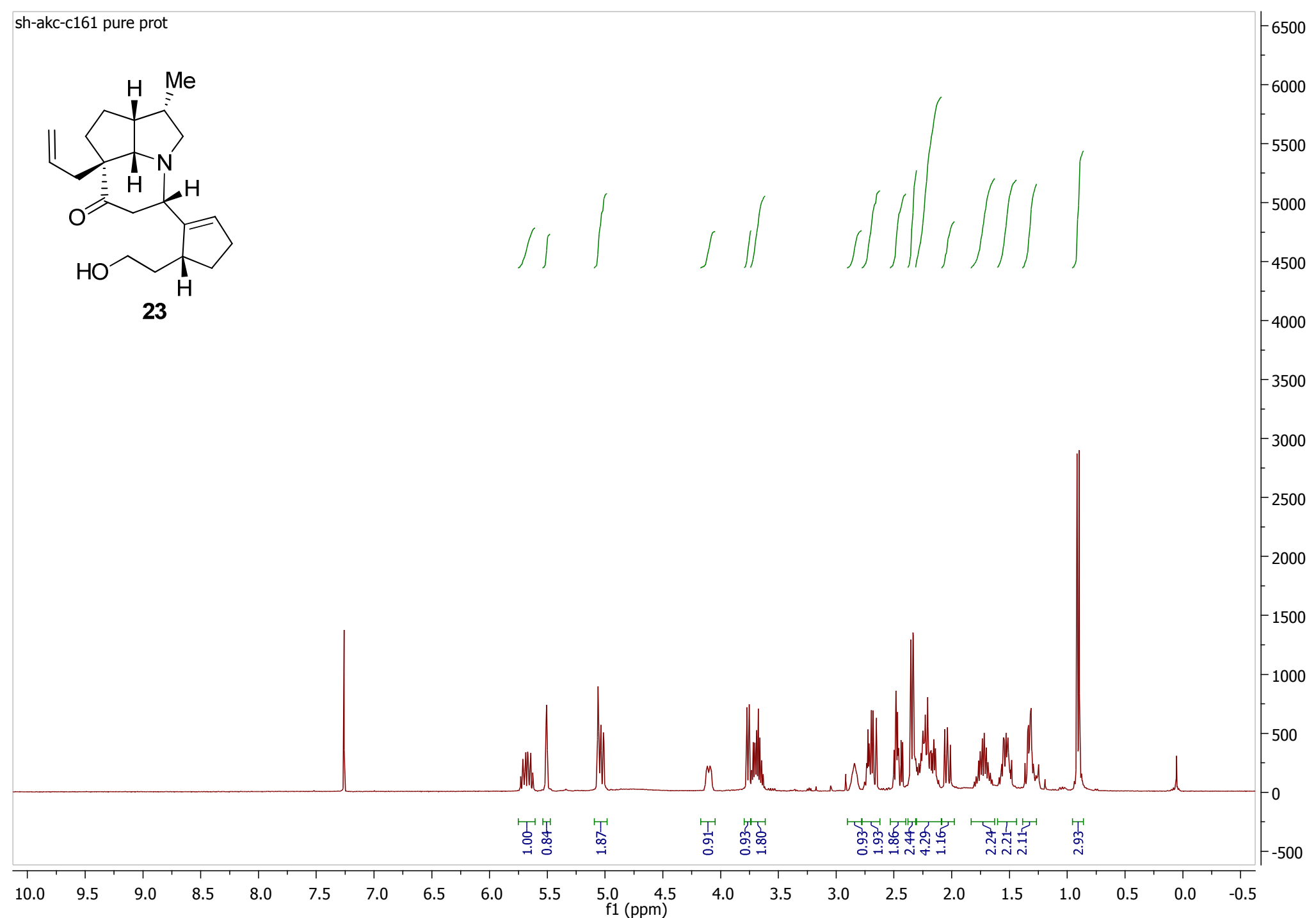

23, ( $\left({ }^{1} \mathrm{H} \mathrm{NMR}, \mathrm{CDCl}_{3}, 400 \mathrm{MHz}\right)$ 


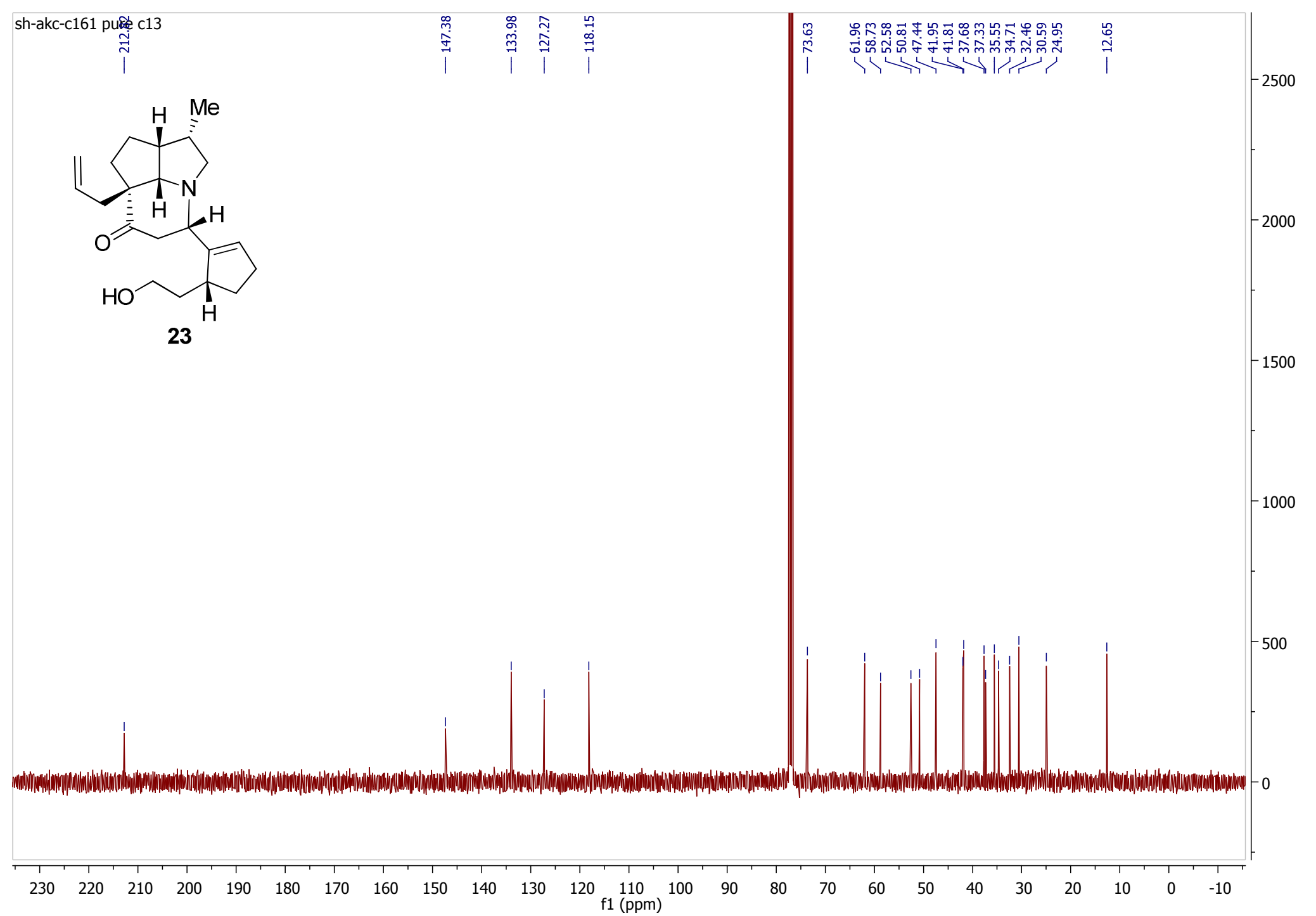

23, $\left({ }^{13} \mathrm{C} \mathrm{NMR}, \mathrm{CDCl}_{3}, 100 \mathrm{MHz}\right)$ 


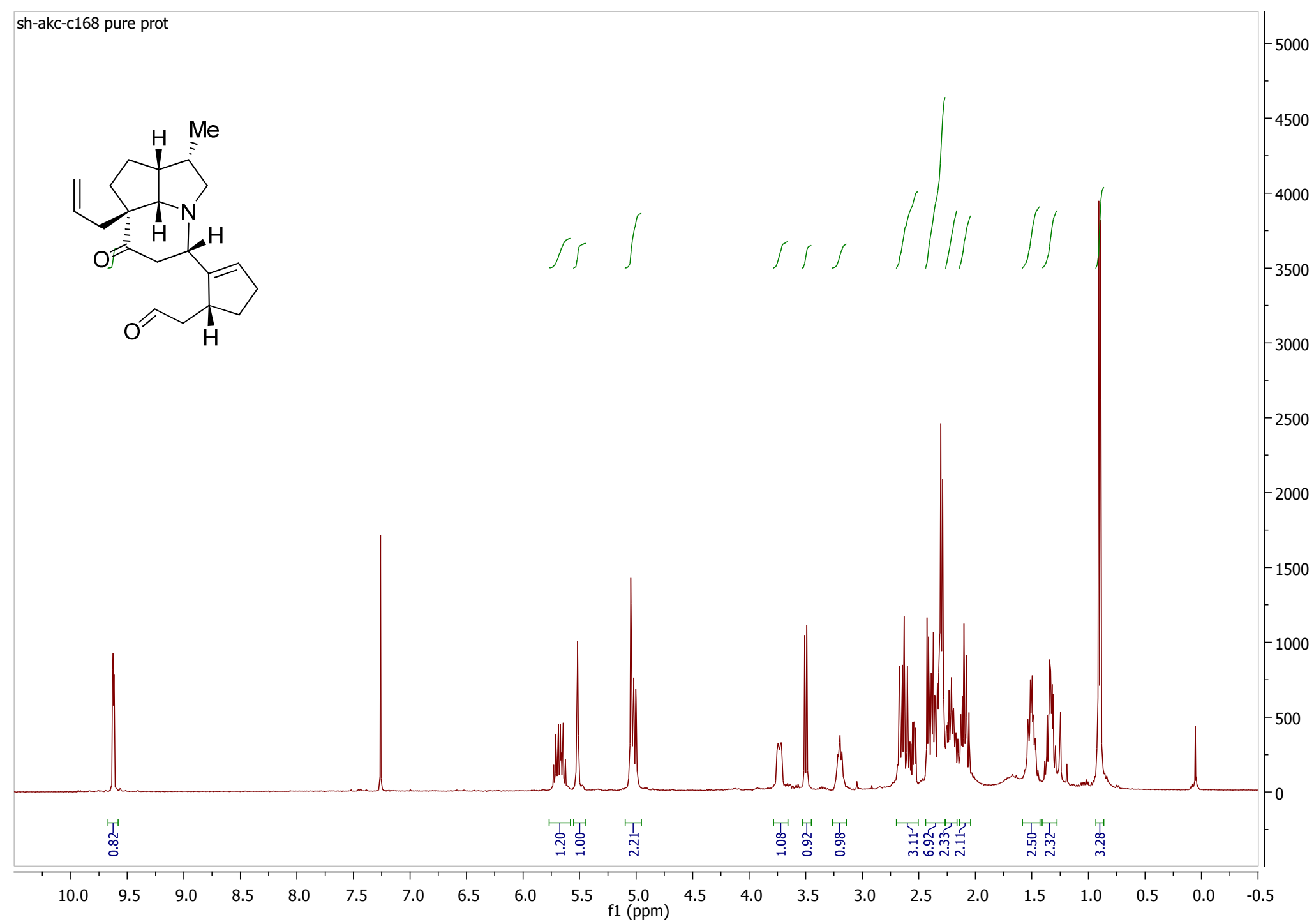

$\left({ }^{1} \mathrm{H} \mathrm{NMR}, \mathrm{CDCl}_{3}, 400 \mathrm{MHz}\right)$ 


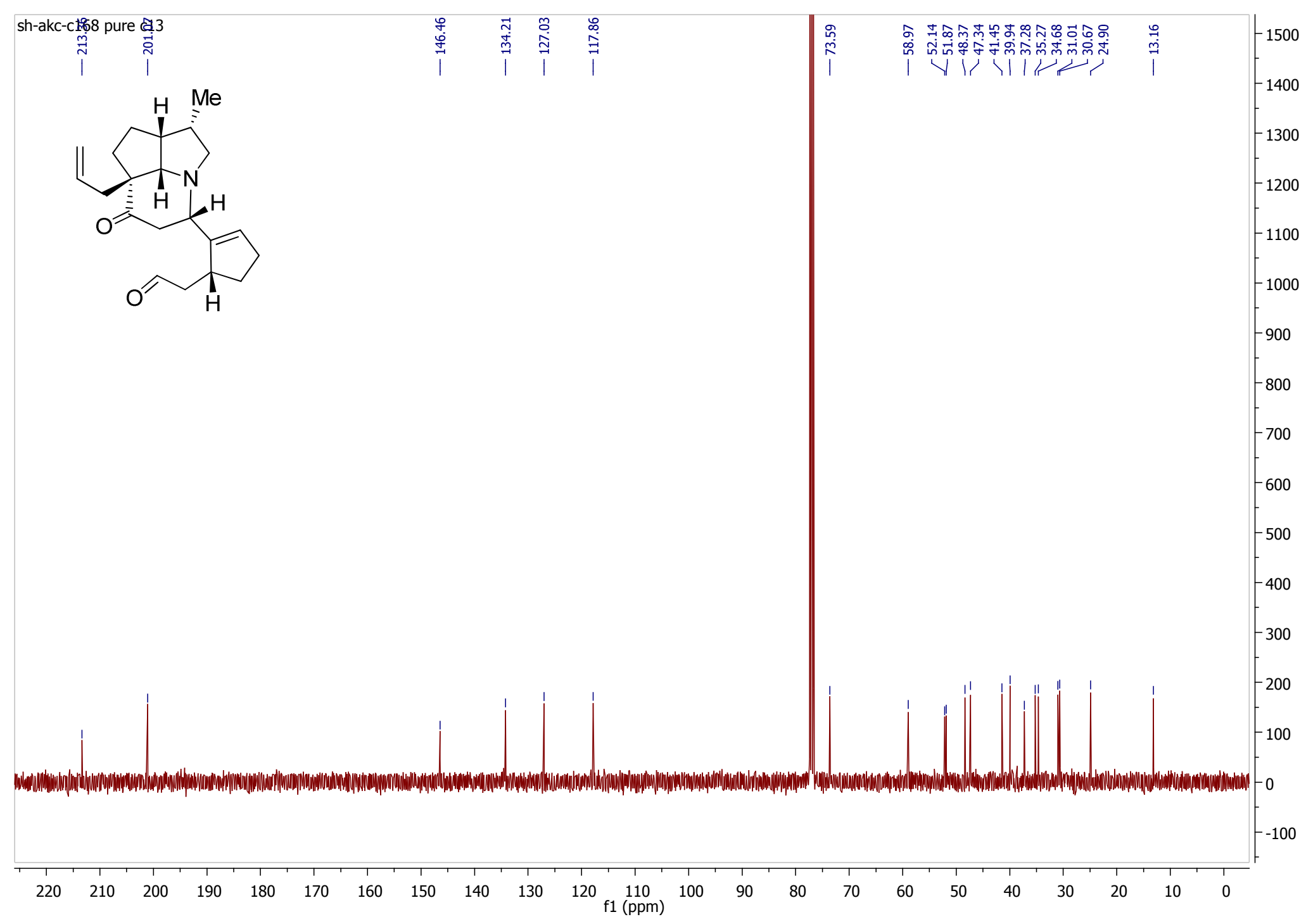

$\left({ }^{13} \mathrm{CNMR} \mathrm{CDCl}_{3}, 100 \mathrm{MHz}\right)$ 


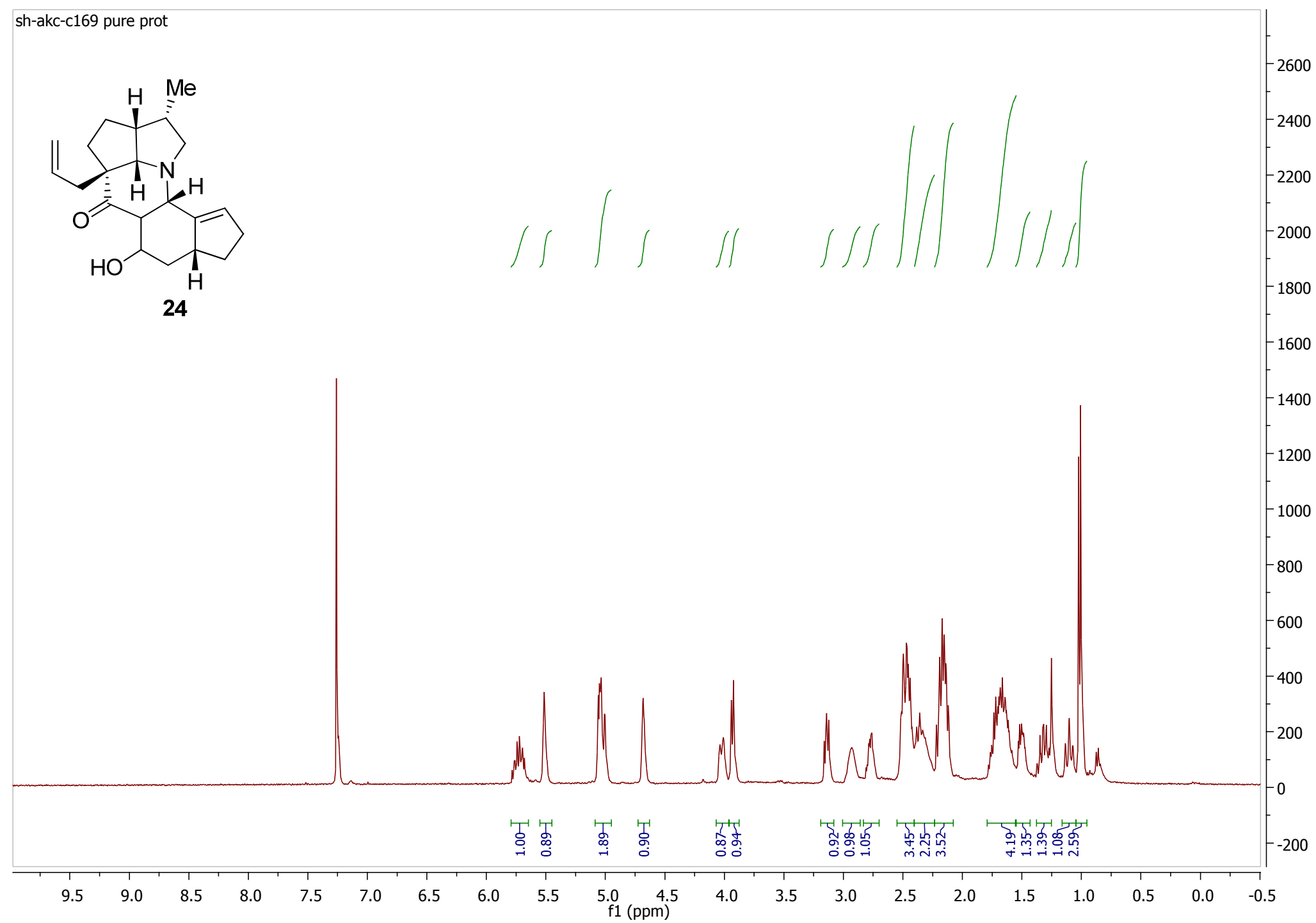

24, $\left({ }^{1} \mathrm{H} N M R, \mathrm{CDCl}_{3}, 400 \mathrm{MHz}\right)$ 


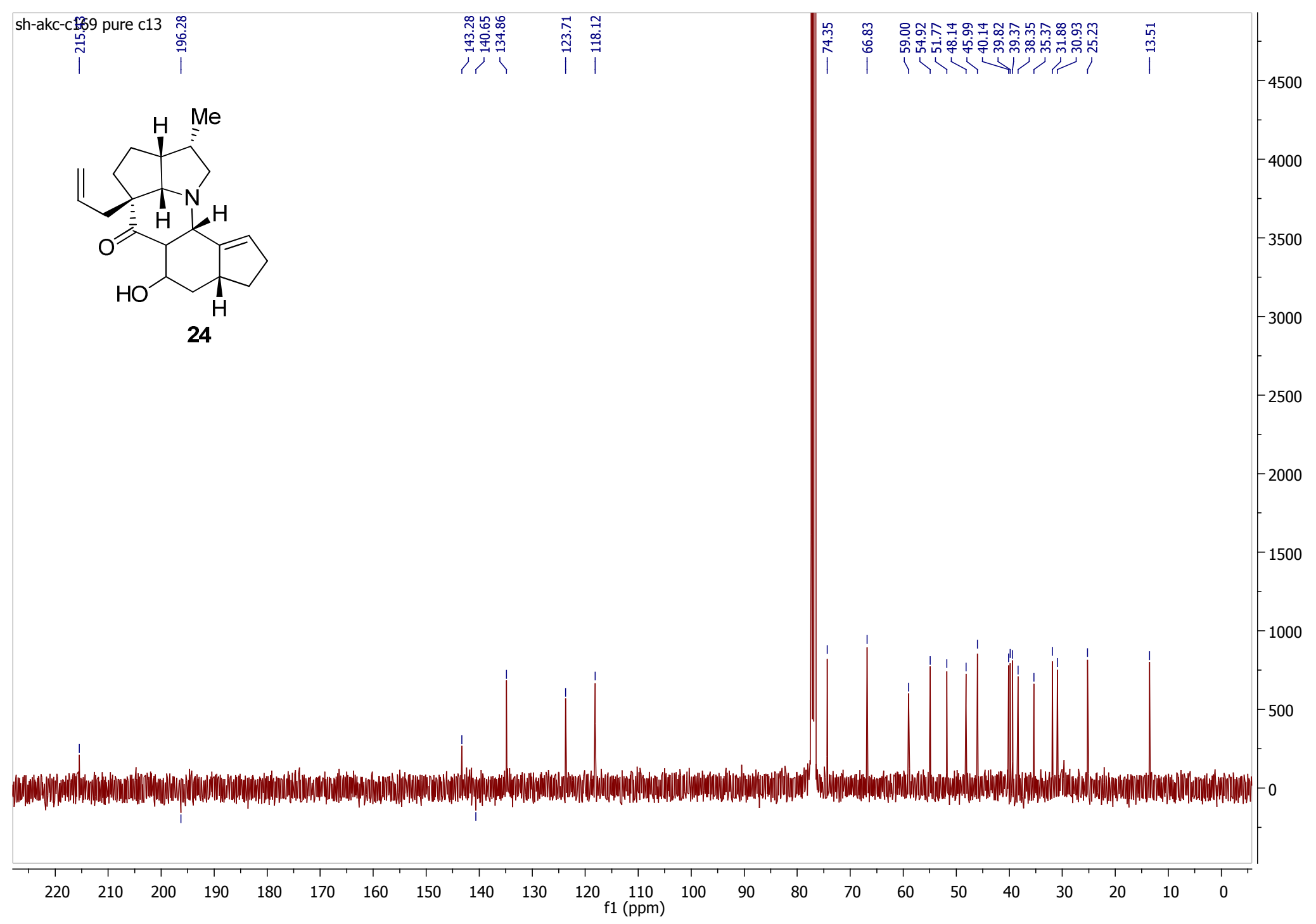

24, $\left({ }^{13} \mathrm{C} \mathrm{NMR}, \mathrm{CDCl}_{3}, 100 \mathrm{MHz}\right)$ 


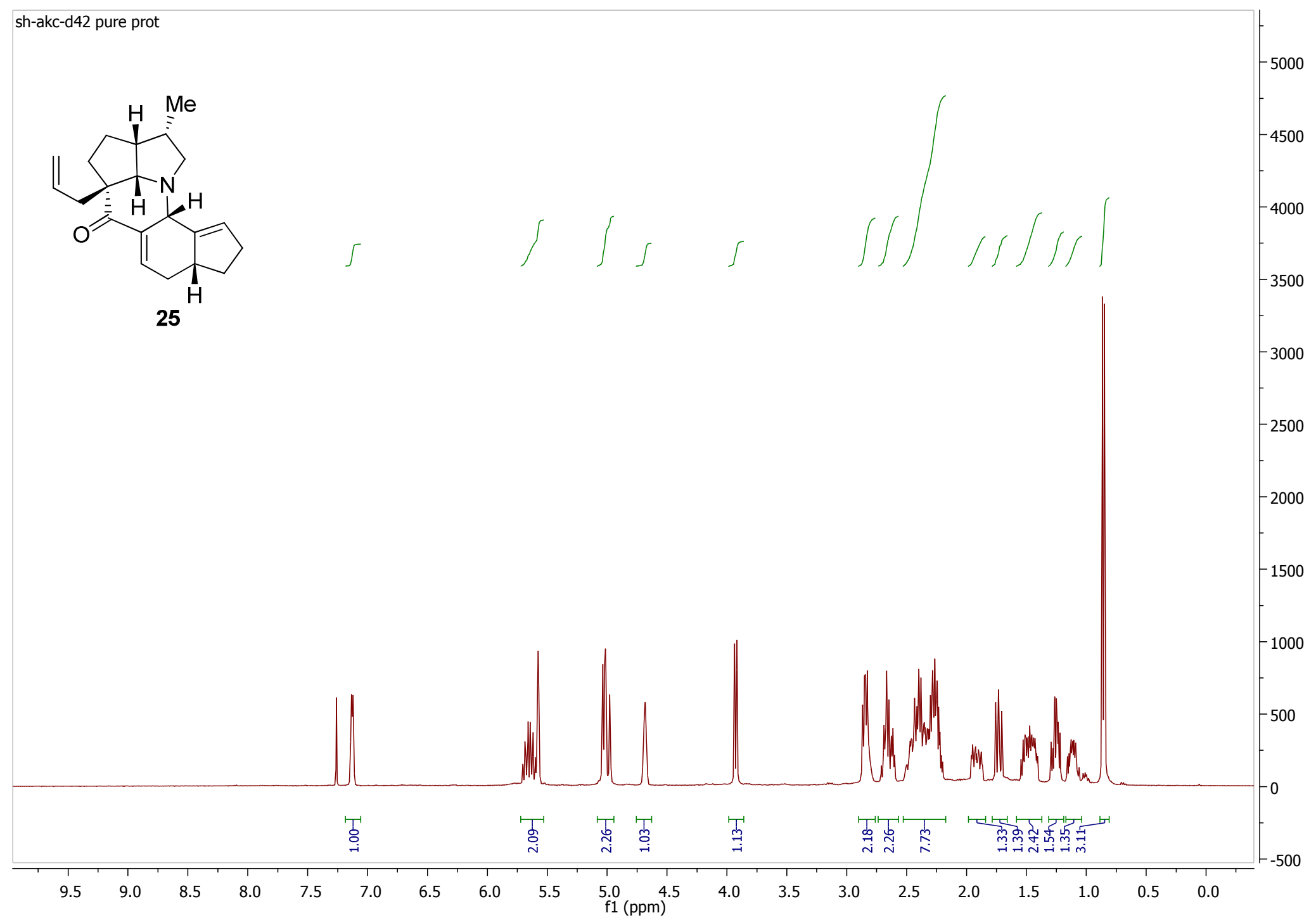

25, ( $\left.{ }^{1} \mathrm{H} N M R, \mathrm{CDCl}_{3}, 400 \mathrm{MHz}\right)$. For X-ray data, see S135-136. 


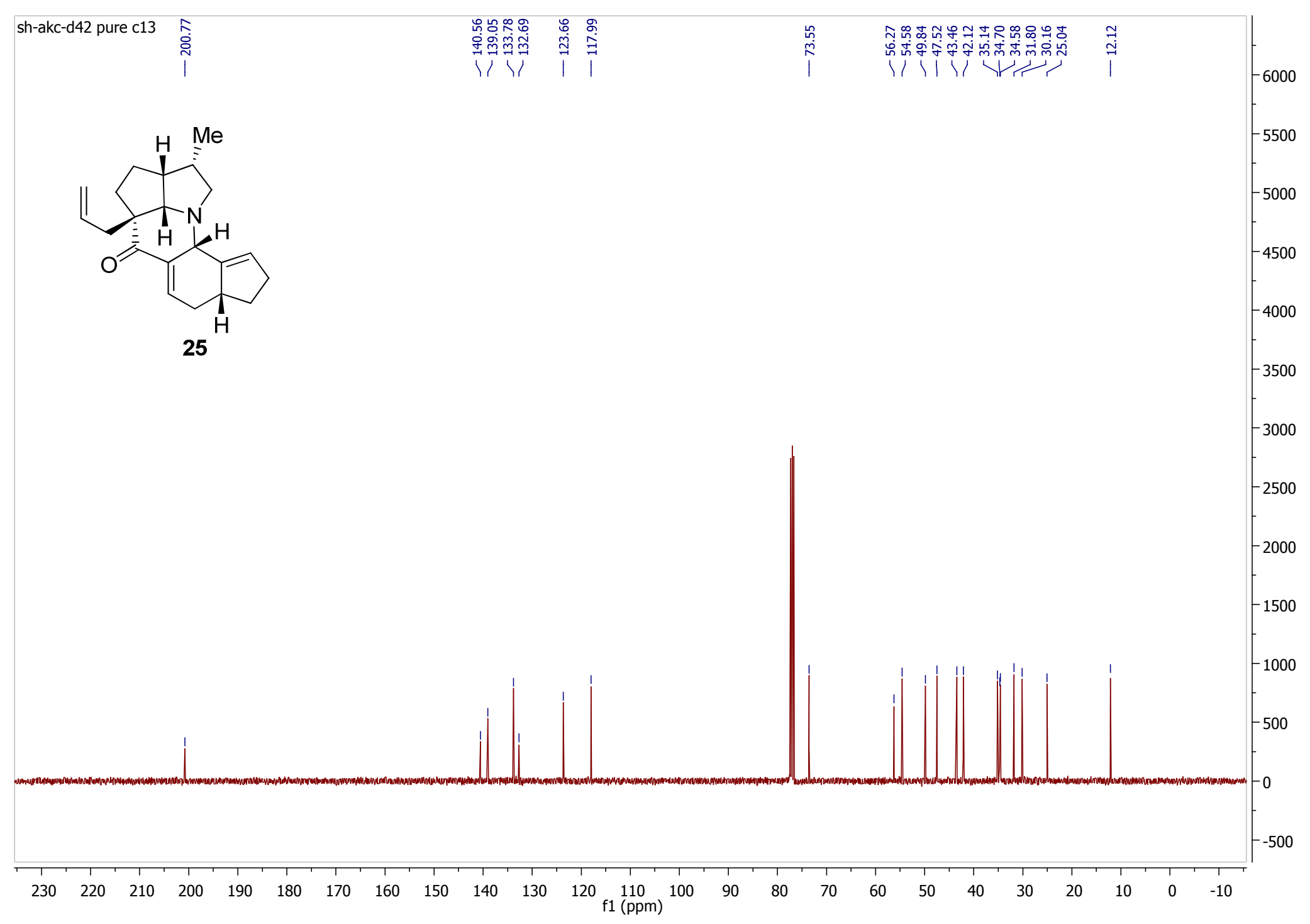

25, $\left({ }^{13} \mathrm{C} \mathrm{NMR}, \mathrm{CDCl}_{3}, 100 \mathrm{MHz}\right)$. For X-ray data, see S135-136. 


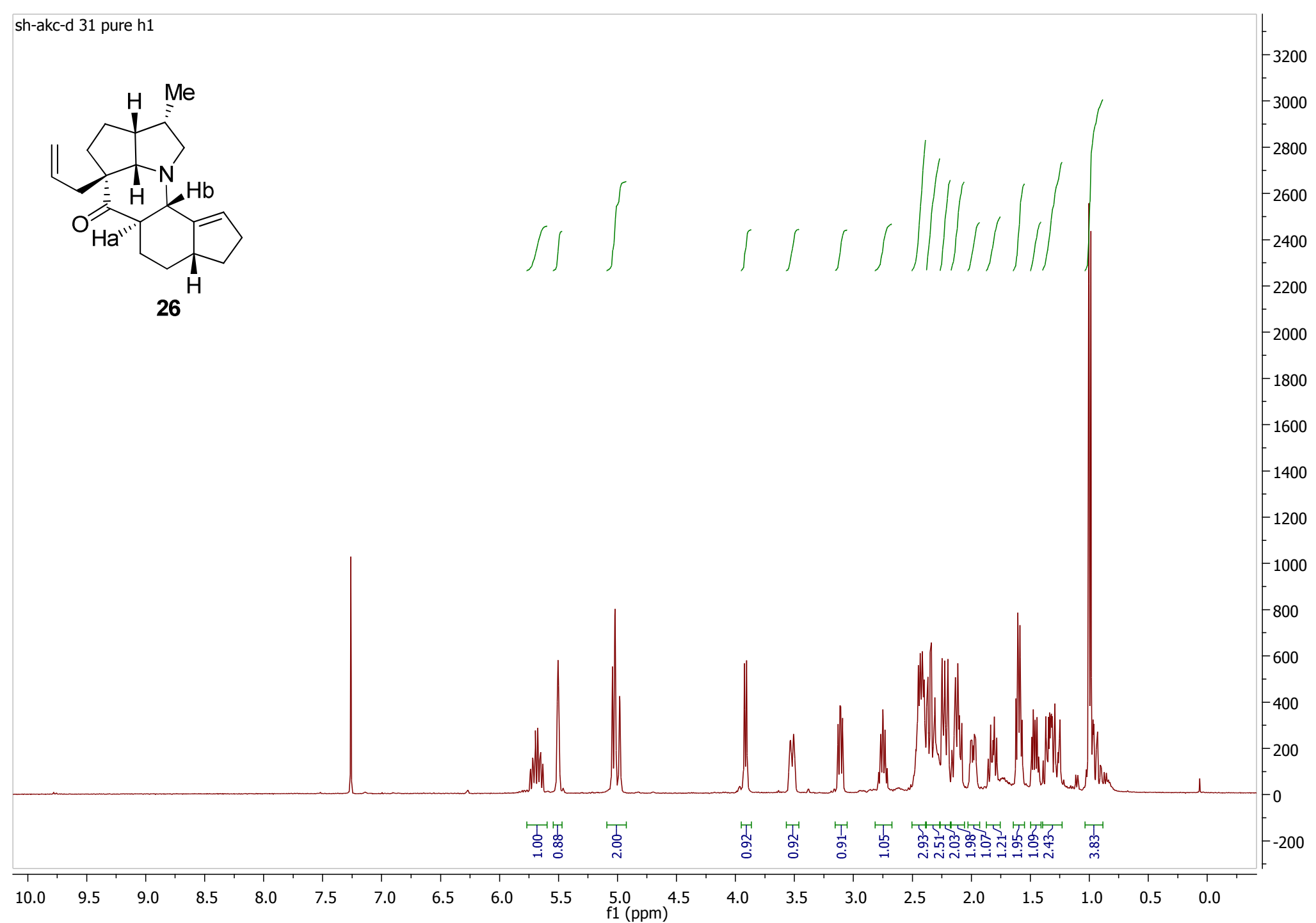

26, $\left({ }^{1} \mathrm{H} \mathrm{NMR}, \mathrm{CDCl}_{3}, 400 \mathrm{MHz}\right.$; Scheme 1, conversion of 25 to 26). For DFT data, see S100. 


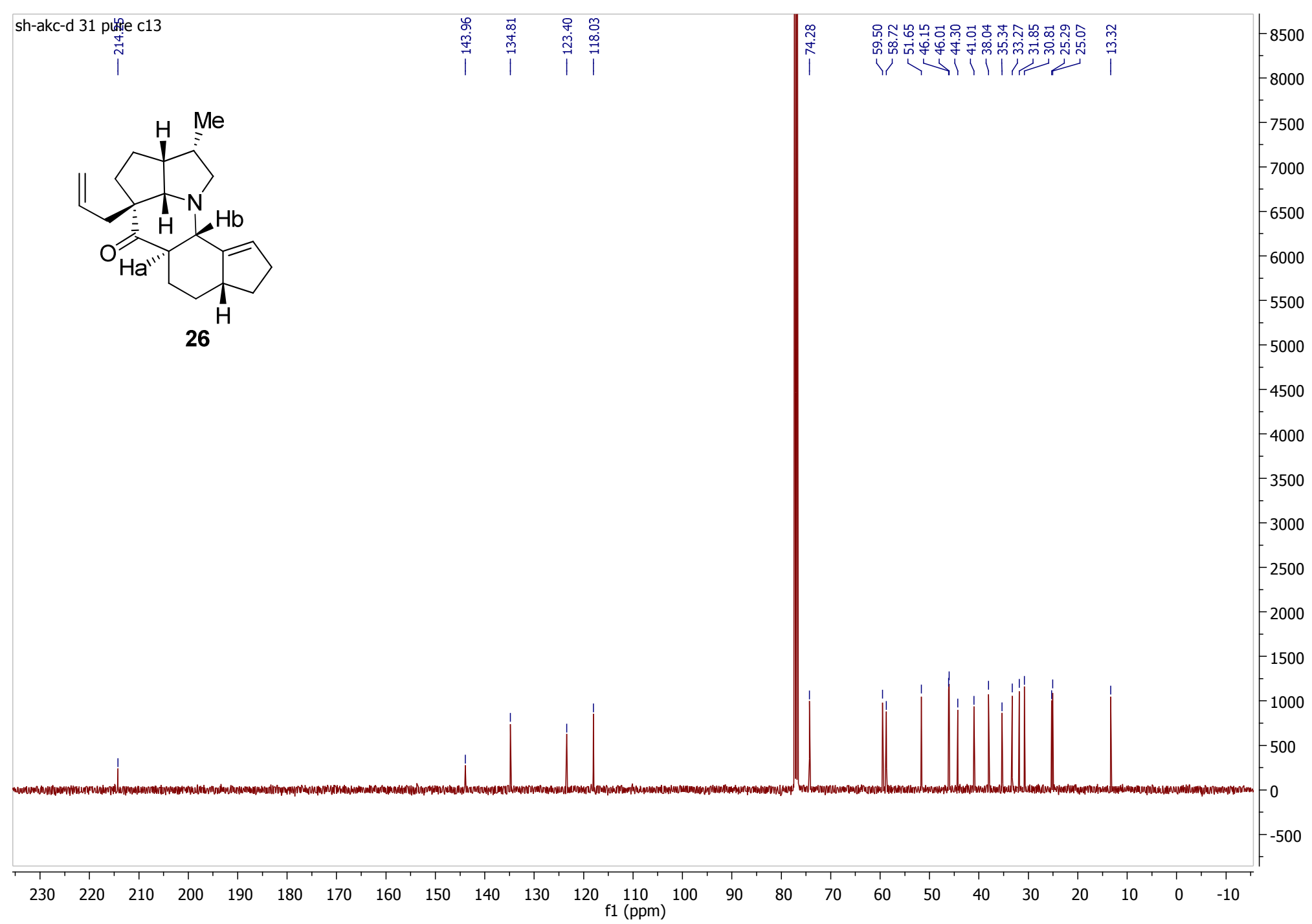

26, $\left({ }^{13} \mathrm{C} \mathrm{NMR}, \mathrm{CDCl}_{3}, 100 \mathrm{MHz}\right.$; Scheme 1, conversion of 25 to 26). For DFT data, see S100. 


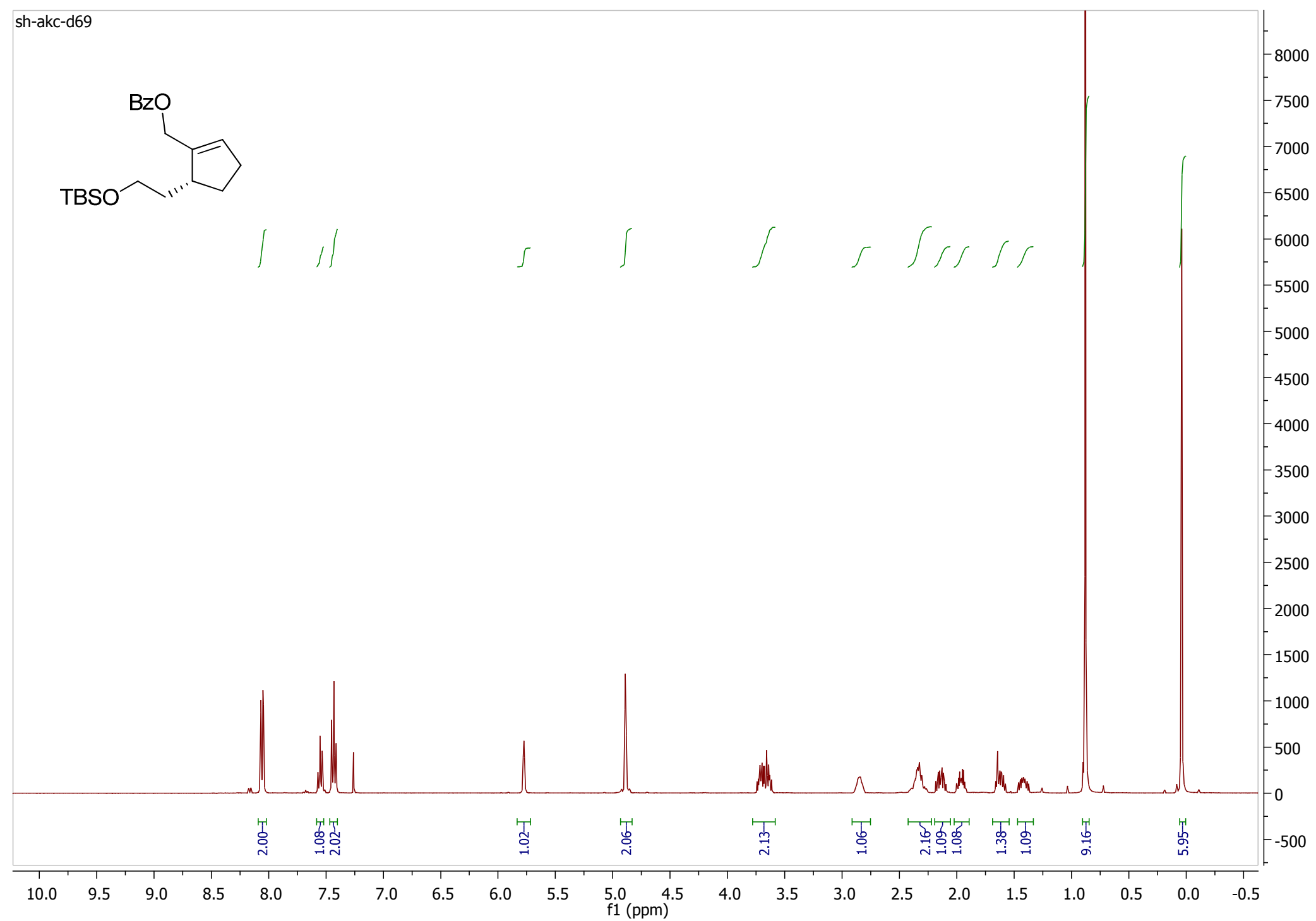

$\left({ }^{1} \mathrm{H} \mathrm{NMR}, \mathrm{CDCl}_{3}, 400 \mathrm{MHz}\right)$ 


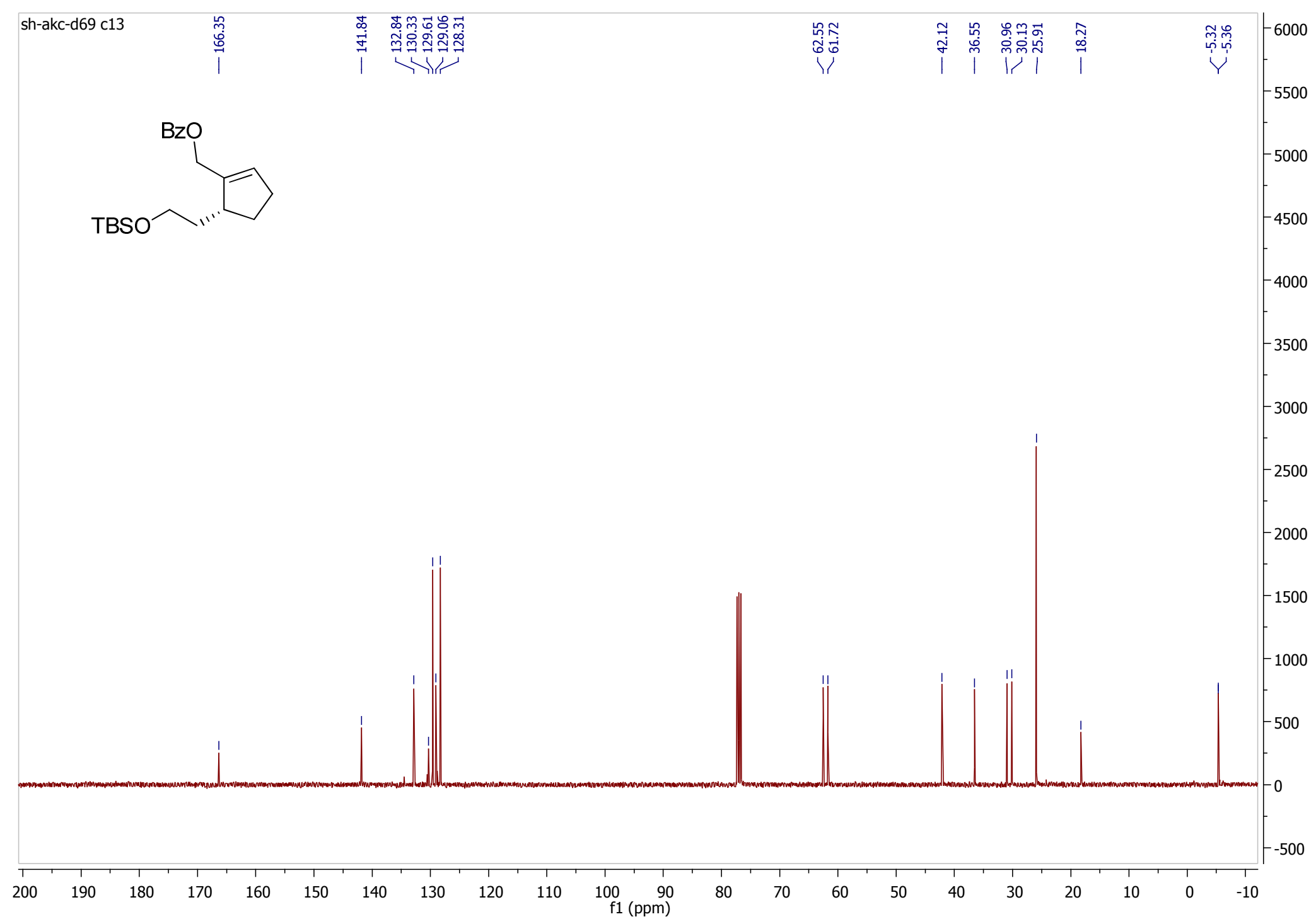

$\left({ }^{13} \mathrm{C} \mathrm{NMR}, \mathrm{CDCl}_{3}, 100 \mathrm{MHz}\right)$ 


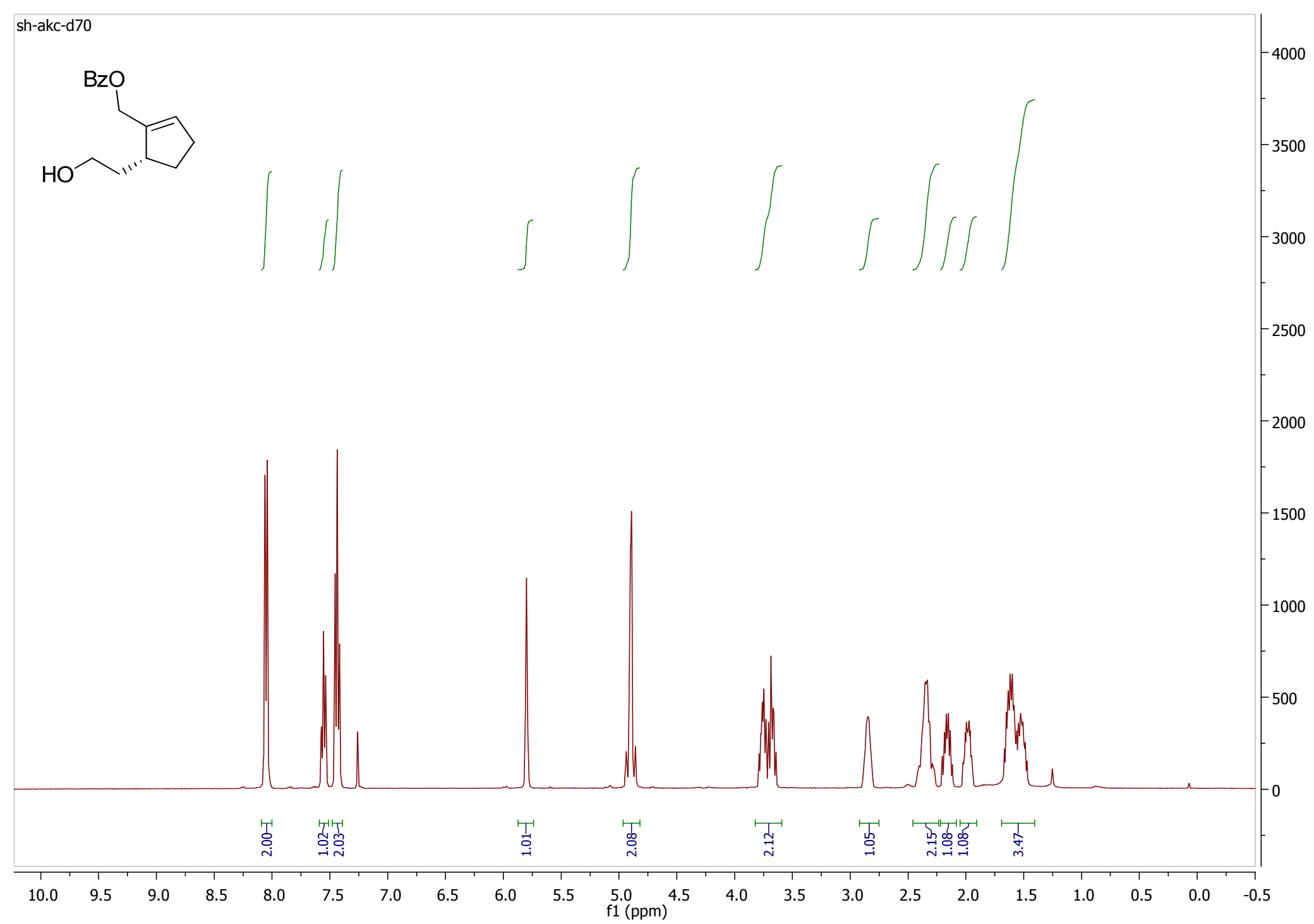

$\left({ }^{1} \mathrm{H} \mathrm{NMR}, \mathrm{CDCl}_{3}, 400 \mathrm{MHz}\right)$ 


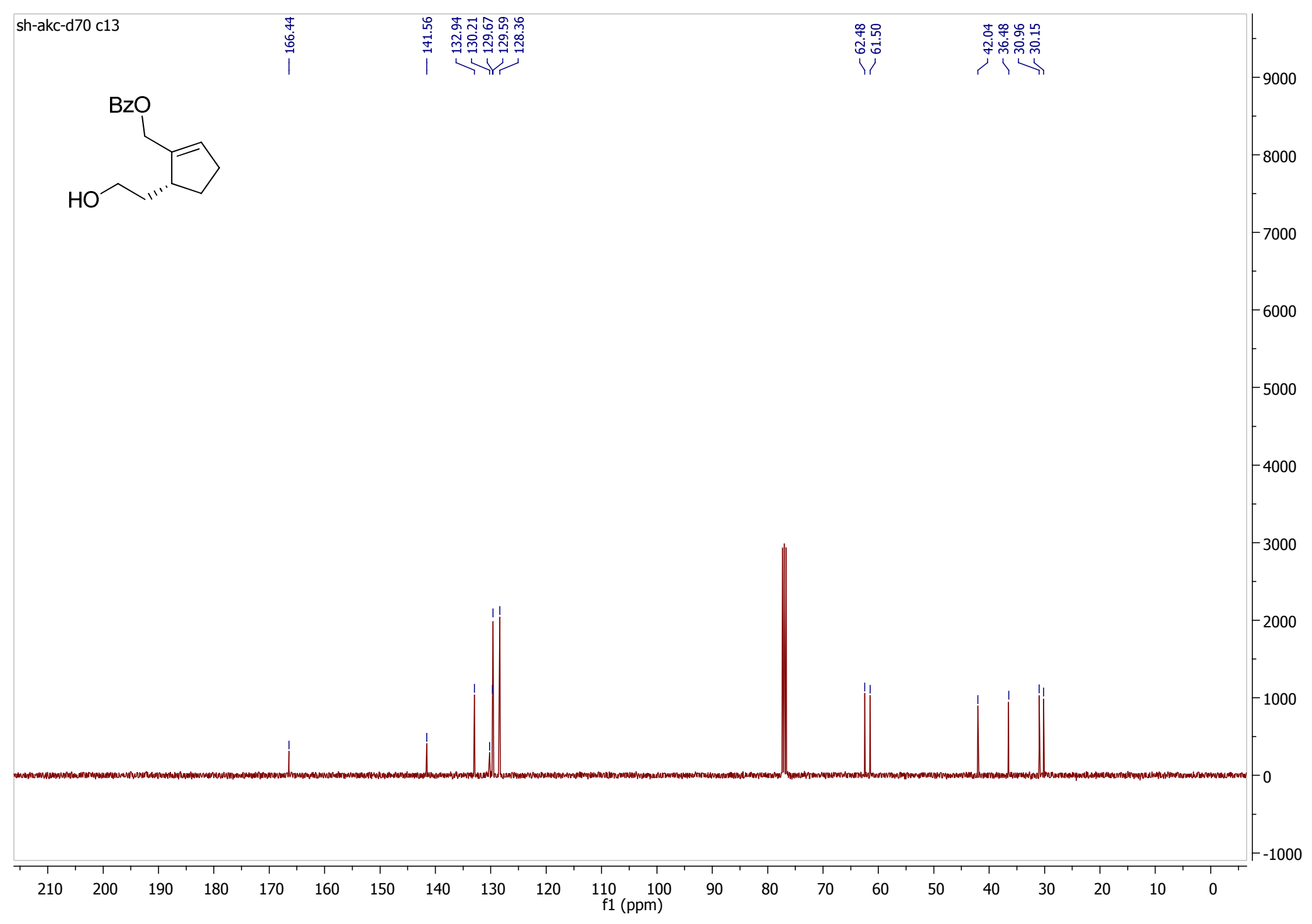

$\left({ }^{13} \mathrm{CNMR}, \mathrm{CDCl}_{3}, 100 \mathrm{MHz}\right)$ 


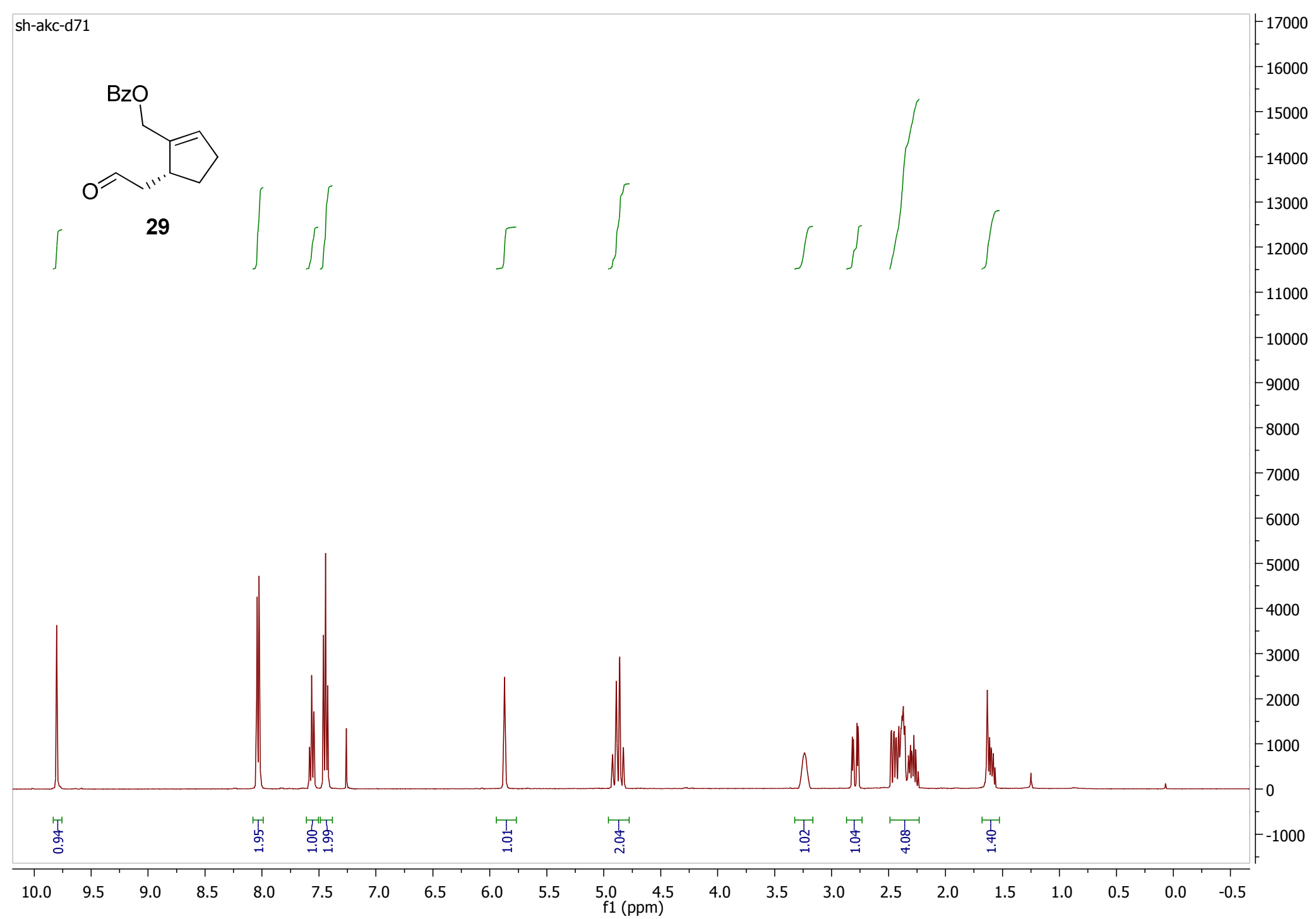

29, ( $\left.{ }^{1} \mathrm{H} \mathrm{NMR}, \mathrm{CDCl}_{3}, 400 \mathrm{MHz}\right)$ 


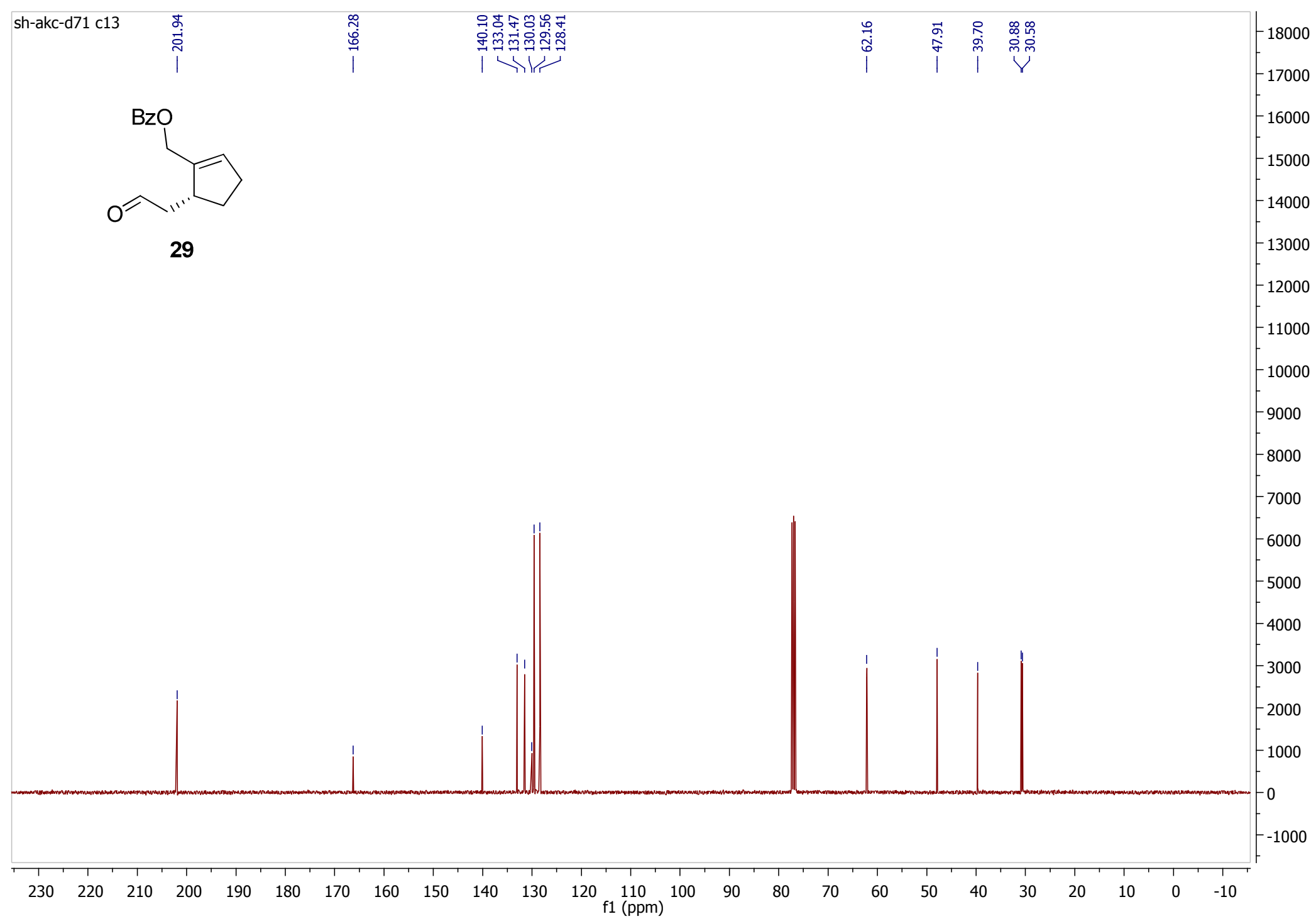

29, $\left({ }^{13} \mathrm{C} \mathrm{NMR}, \mathrm{CDCl}_{3}, 100 \mathrm{MHz}\right)$ 


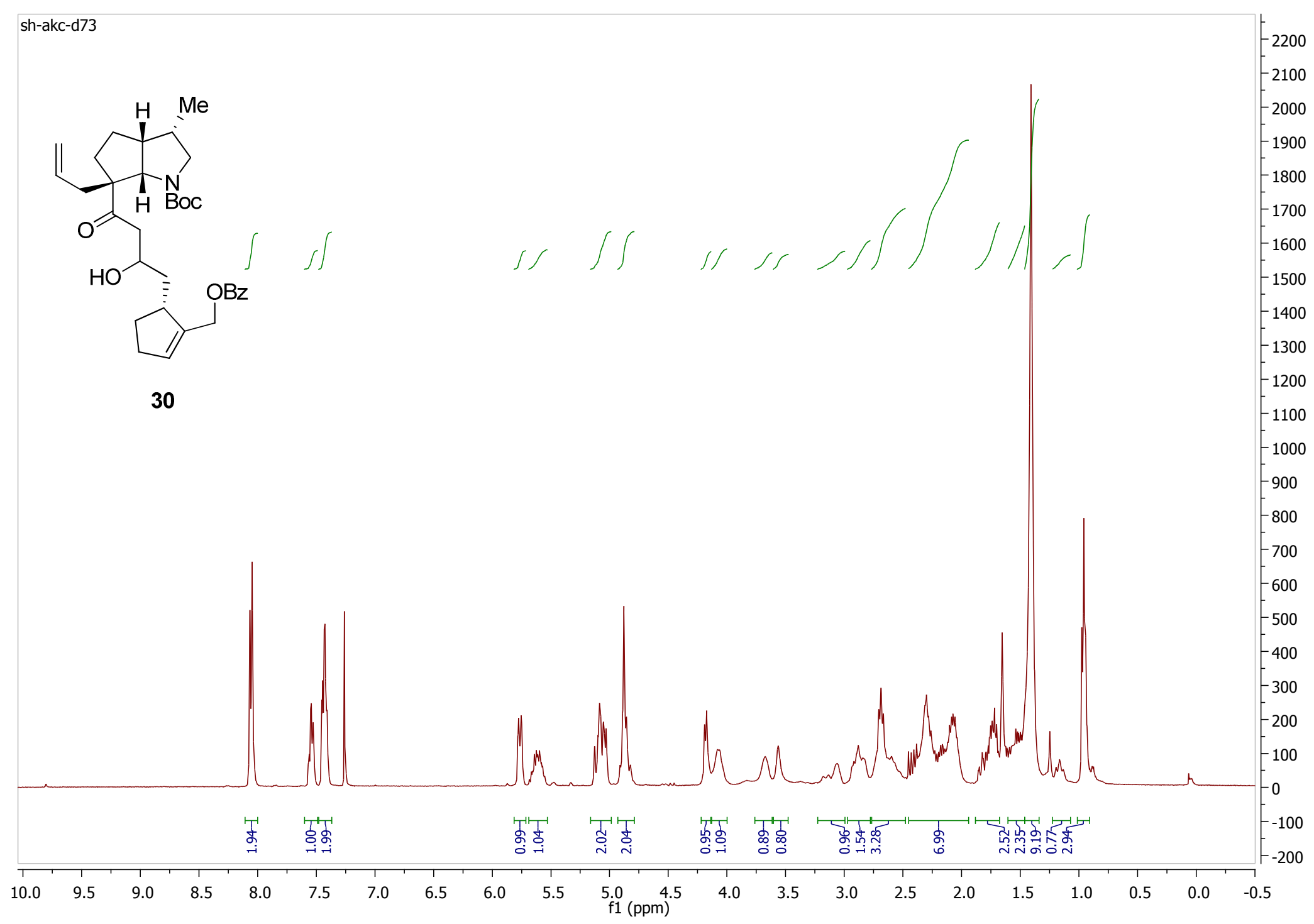

30, $\left({ }^{1} \mathrm{H} \mathrm{NMR}, \mathrm{CDCl}_{3}, 400 \mathrm{MHz}\right)$ 


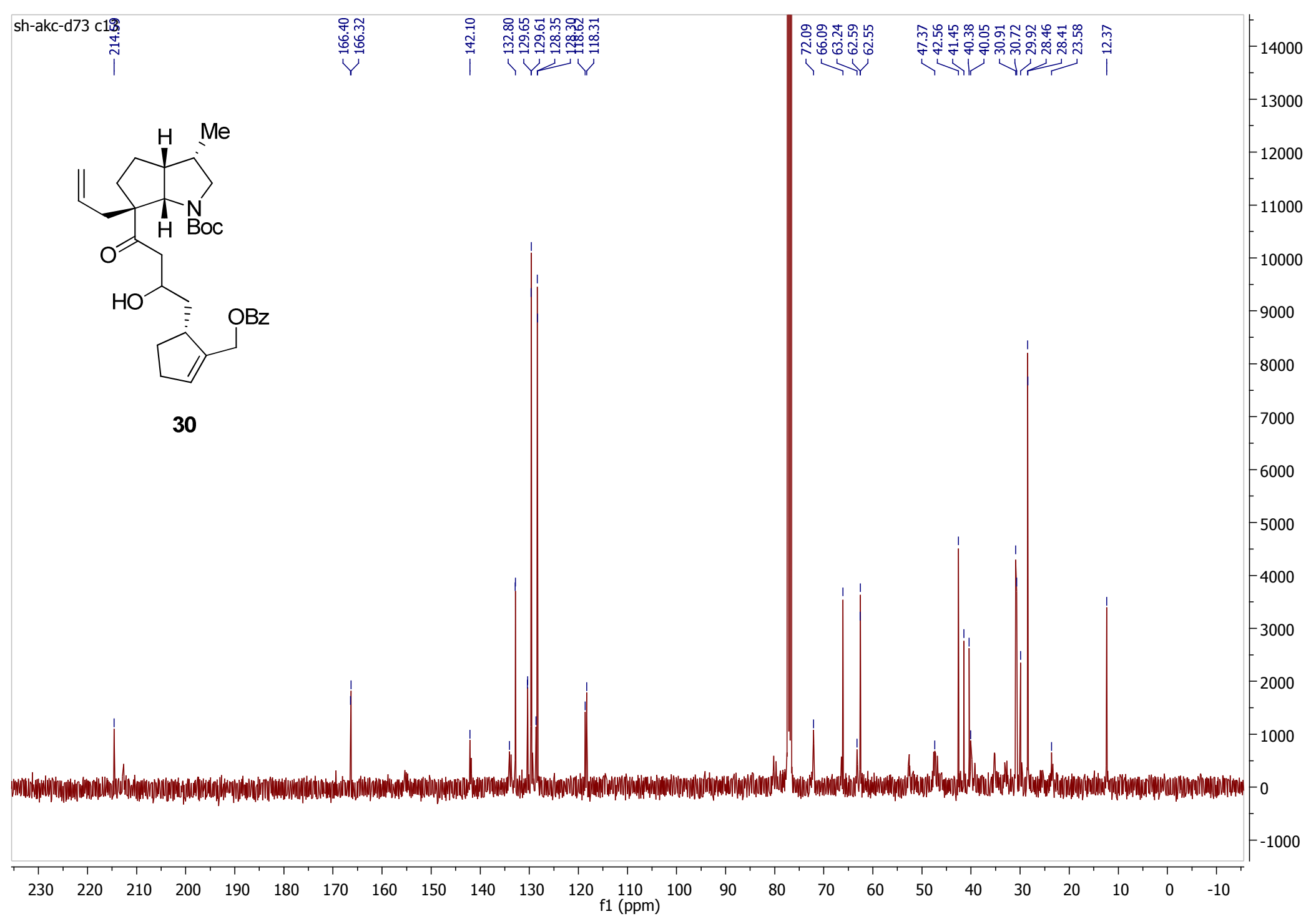

30, $\left({ }^{13} \mathrm{C} \mathrm{NMR}, \mathrm{CDCl}_{3}, 100 \mathrm{MHz}\right)$ 


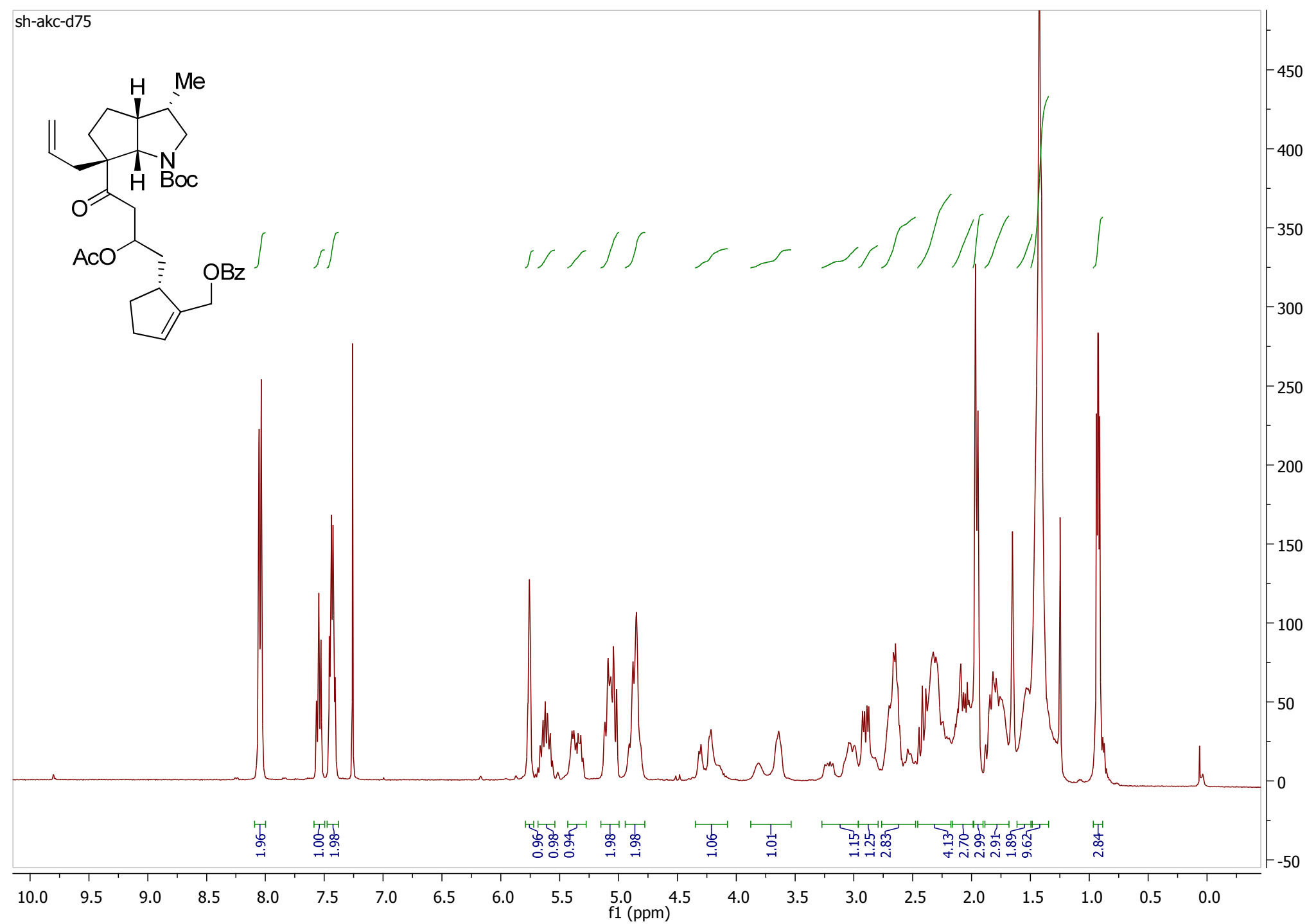

$\left({ }^{1} \mathrm{H} \mathrm{NMR}, \mathrm{CDCl}_{3}, 400 \mathrm{MHz}\right)$ 


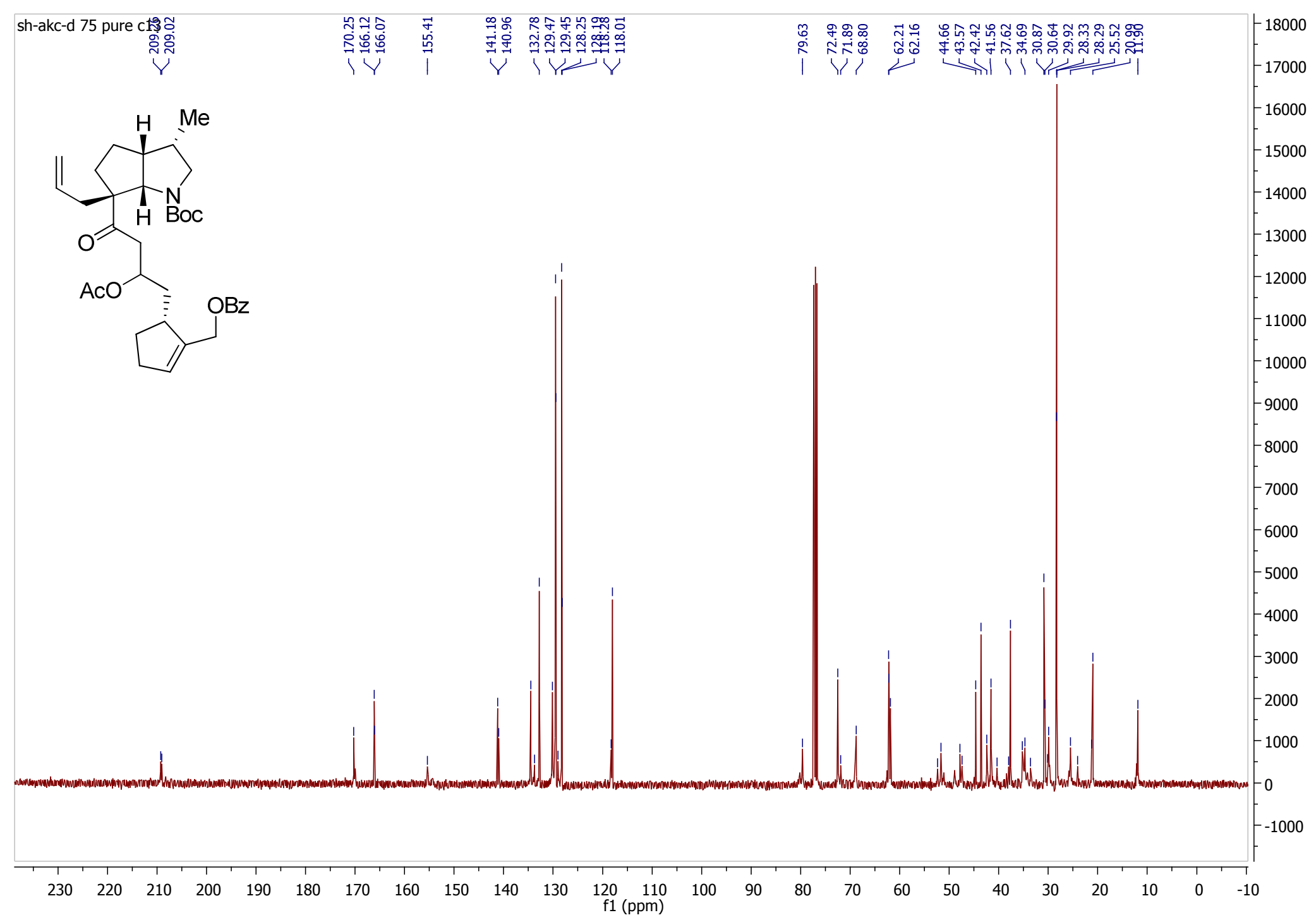

$\left({ }^{13} \mathrm{C} \mathrm{NMR}, \mathrm{CDCl}_{3}, 100 \mathrm{MHz}\right)$ 


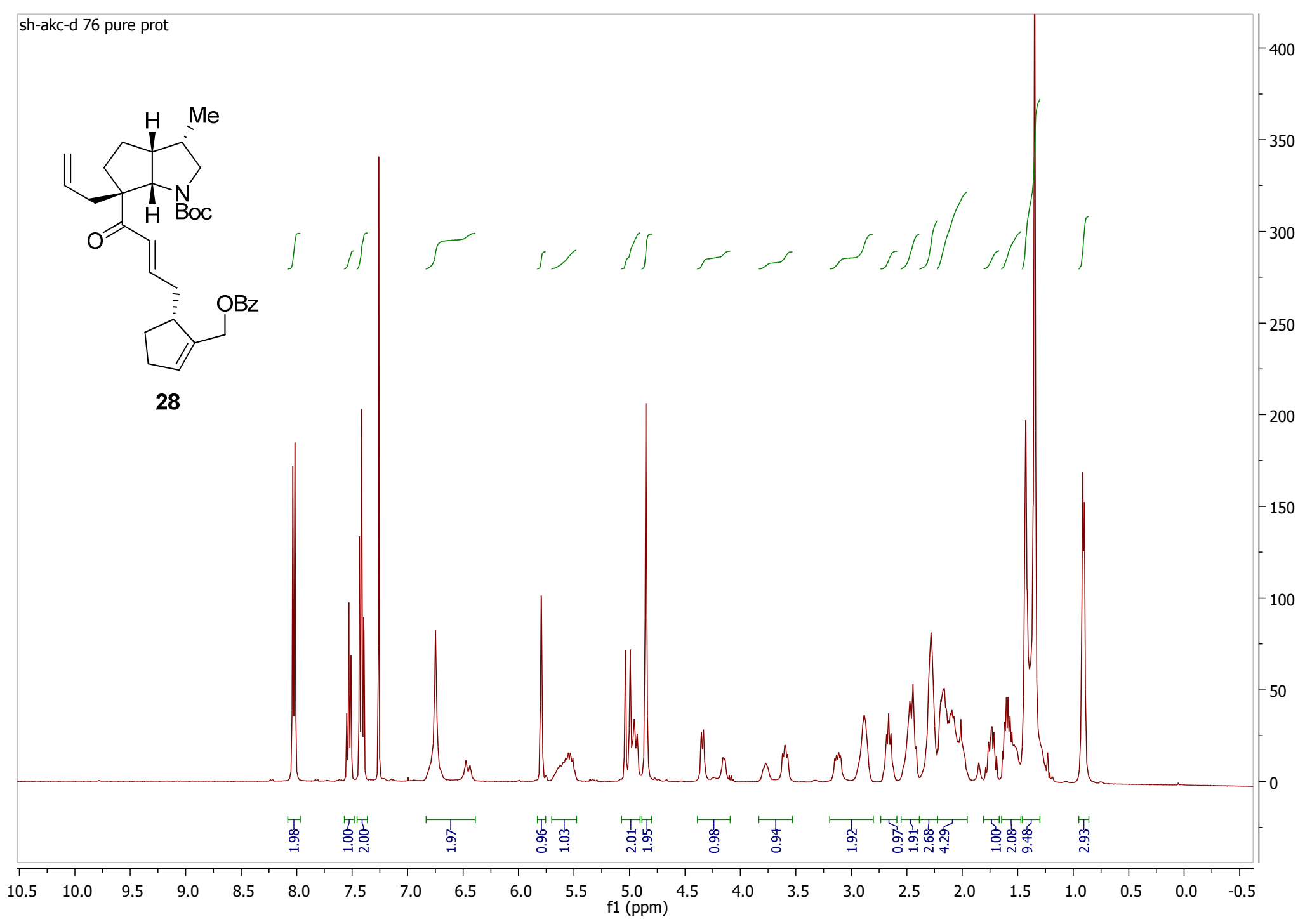

28, ( $\left.{ }^{1} \mathrm{H} N M R, \mathrm{CDCl}_{3}, 400 \mathrm{MHz}\right)$ 


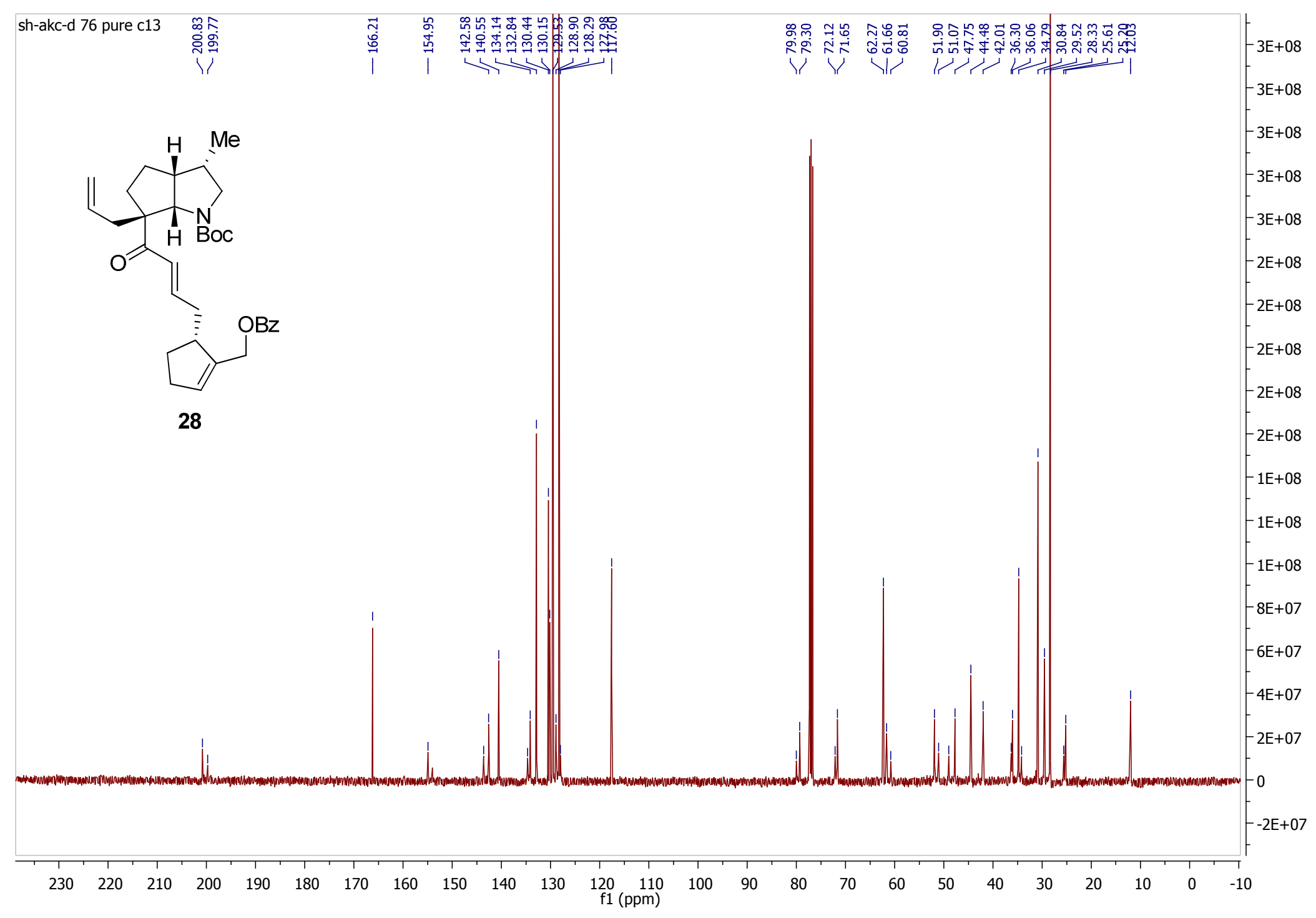

28, $\left({ }^{13} \mathrm{C} \mathrm{NMR}, \mathrm{CDCl}_{3}, 100 \mathrm{MHz}\right)$ 


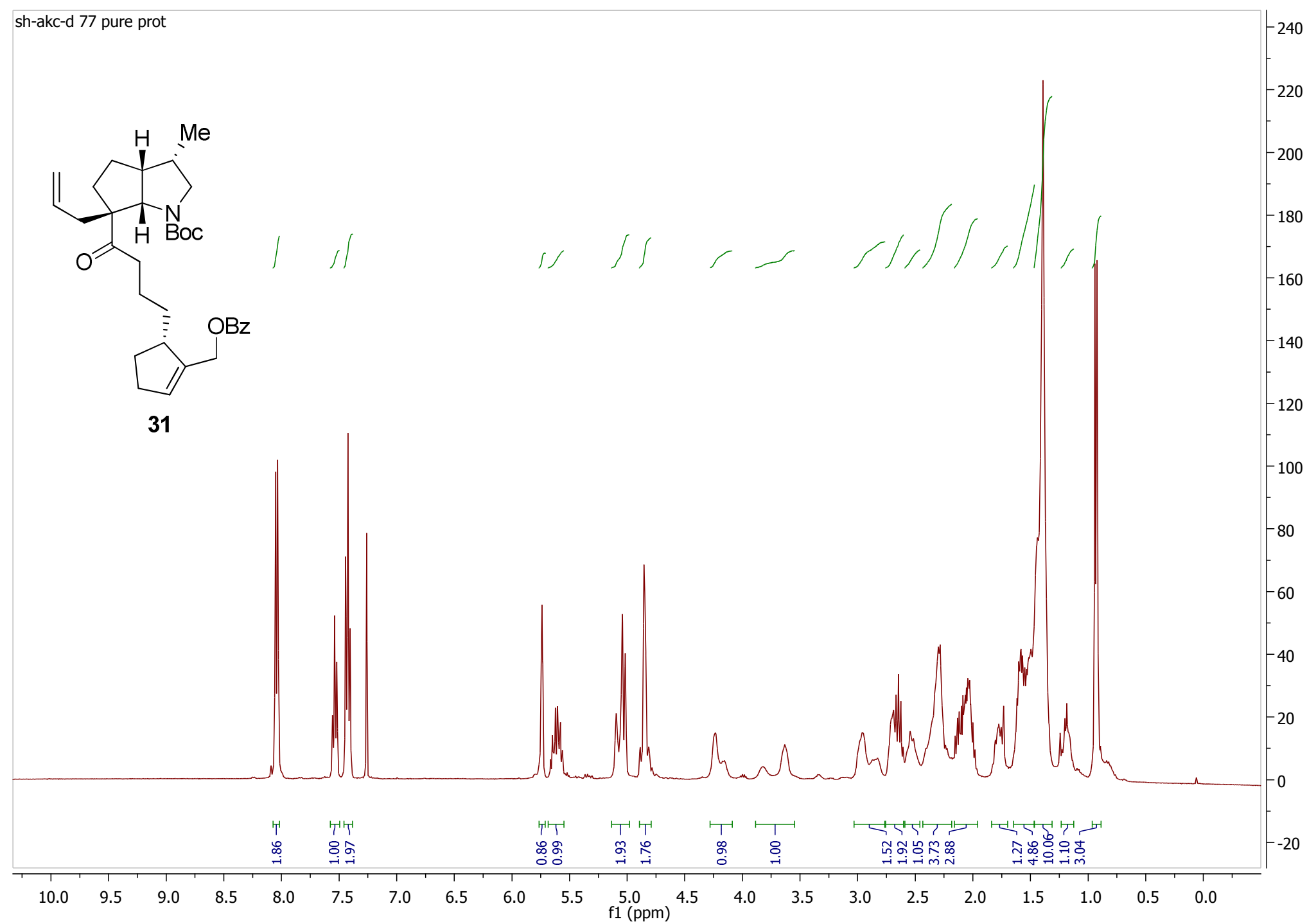

31, ( $\left.{ }^{1} \mathrm{H} N M R, \mathrm{CDCl}_{3}, 400 \mathrm{MHz}\right)$ 


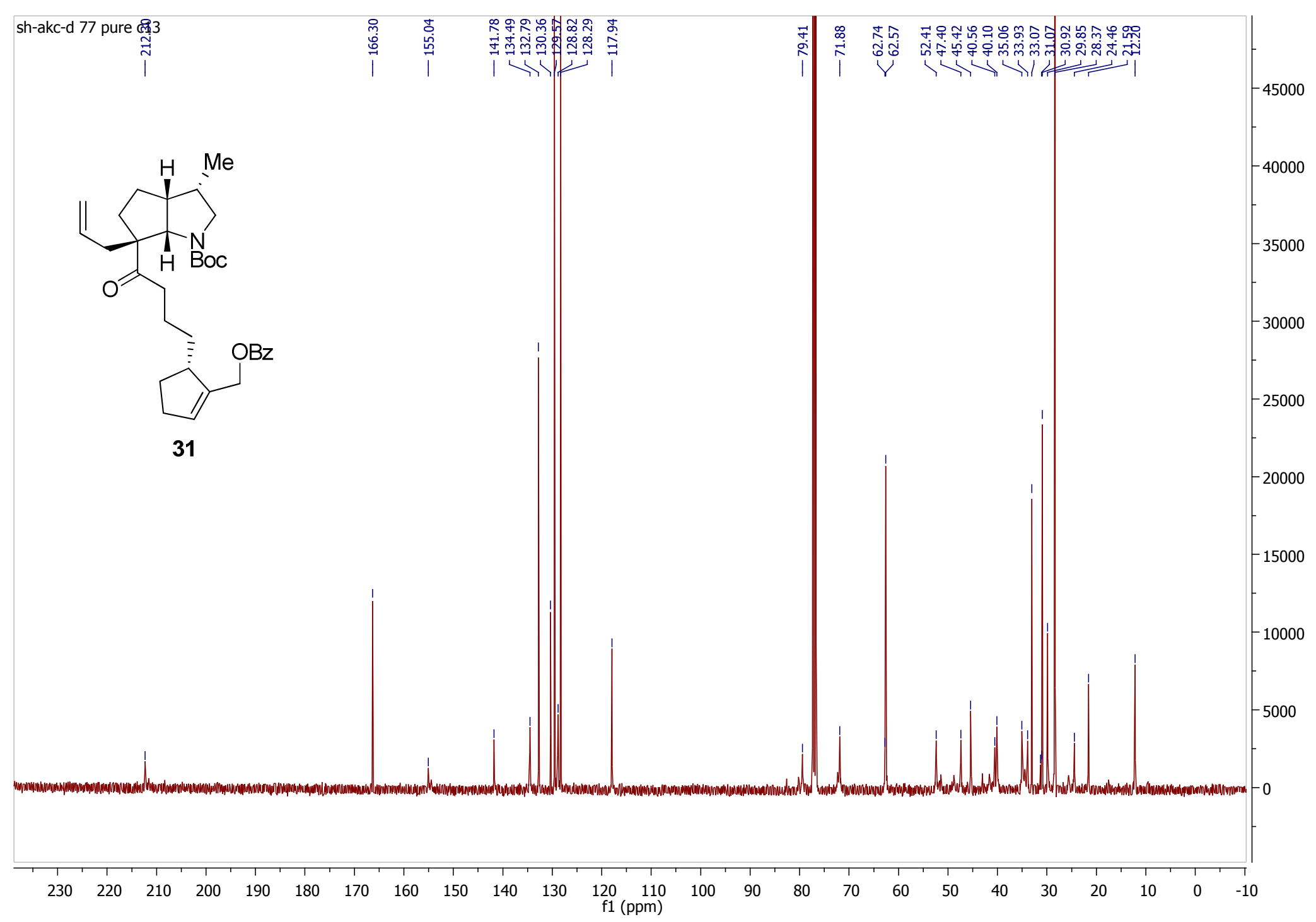

31, $\left({ }^{13} \mathrm{C} \mathrm{NMR}, \mathrm{CDCl}_{3}, 100 \mathrm{MHz}\right)$ 


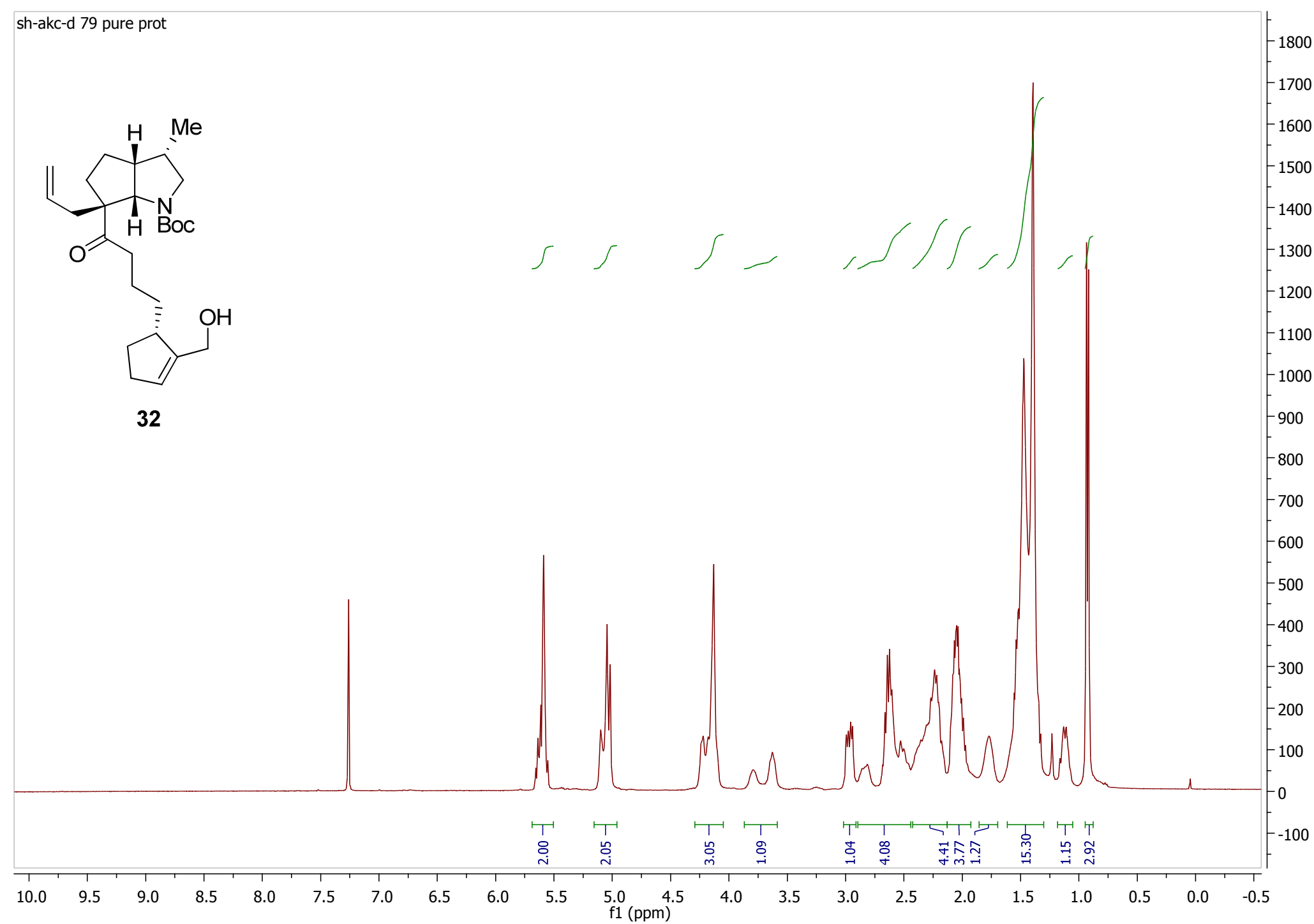

32, $\left({ }^{1} \mathrm{H} N M R, \mathrm{CDCl}_{3}, 400 \mathrm{MHz}\right)$ 


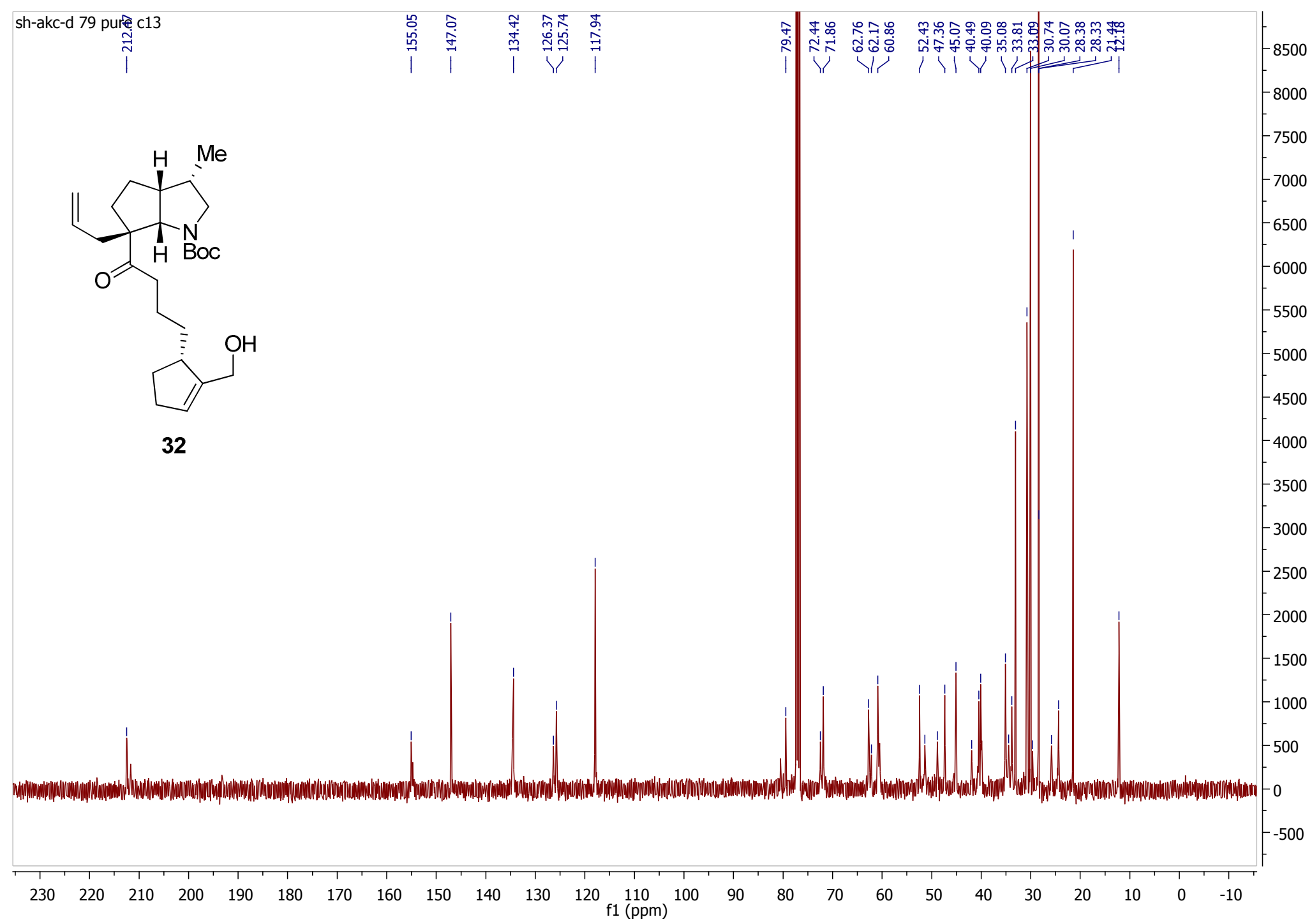

32, $\left({ }^{13} \mathrm{C} \mathrm{NMR}, \mathrm{CDCl}_{3}, 100 \mathrm{MHz}\right)$ 


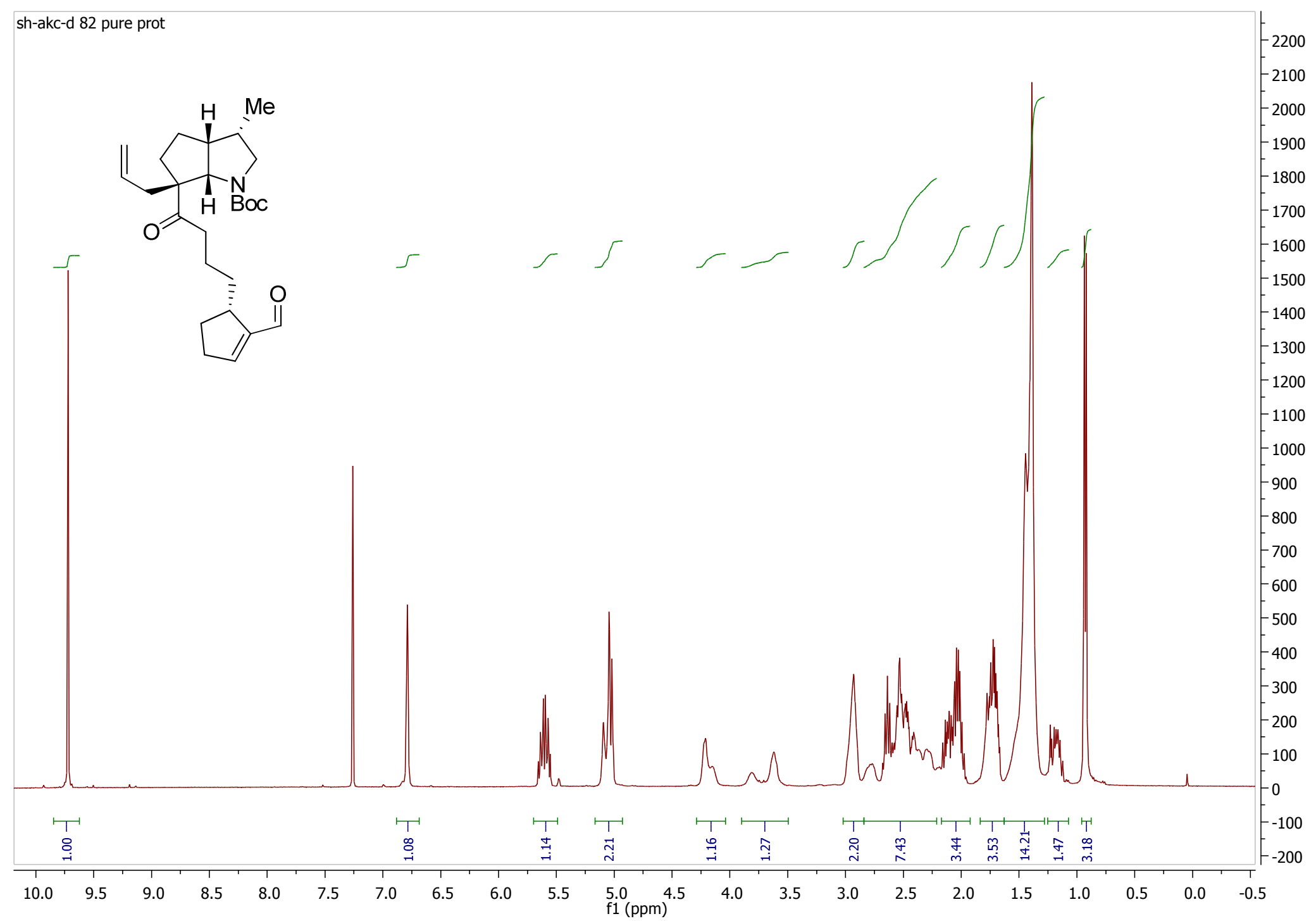

$\left({ }^{1} \mathrm{H} \mathrm{NMR}, \mathrm{CDCl}_{3}, 400 \mathrm{MHz}\right)$ 


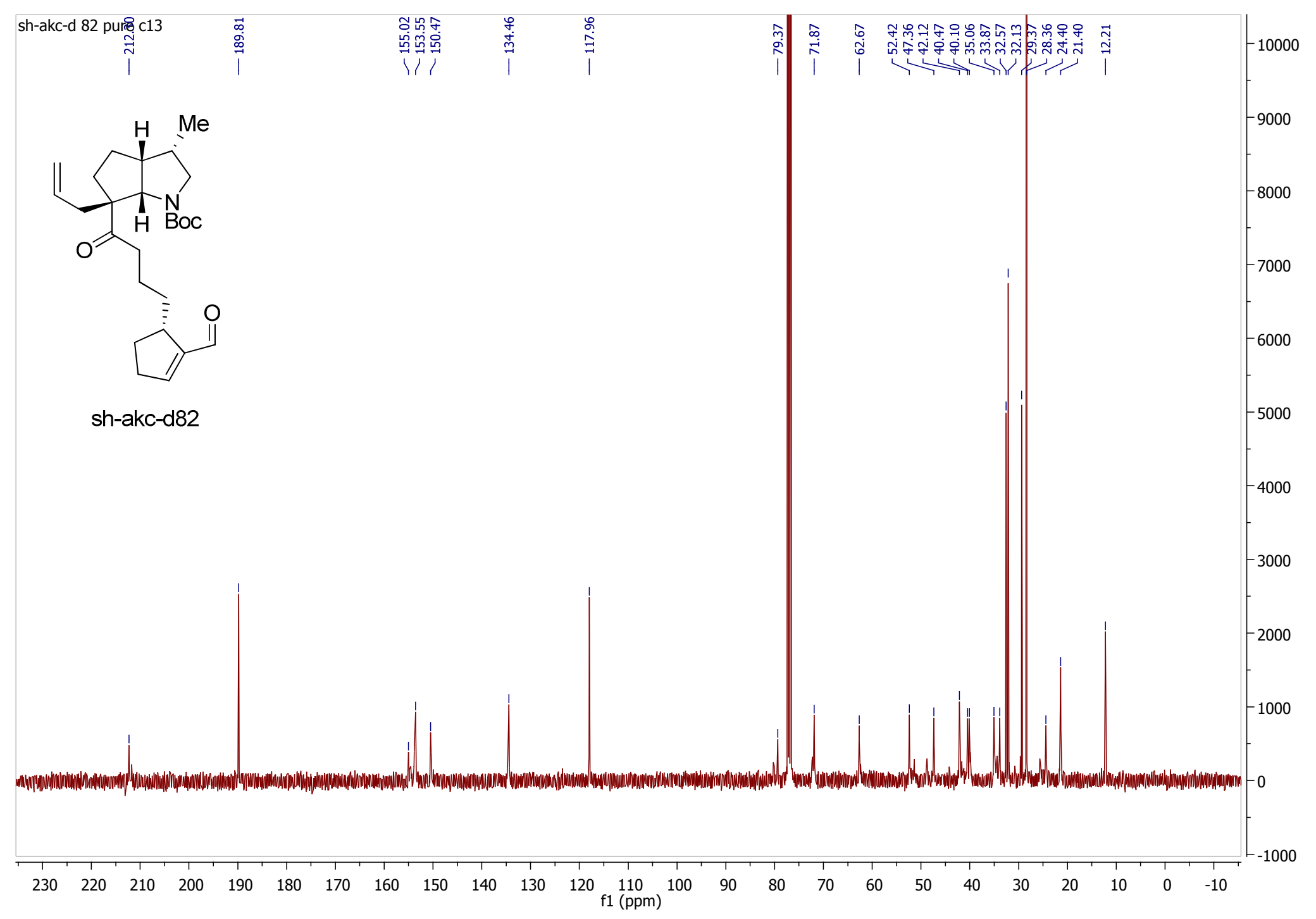

$\left({ }^{13} \mathrm{CNMR} \mathrm{CDCl}_{3}, 100 \mathrm{MHz}\right)$ 


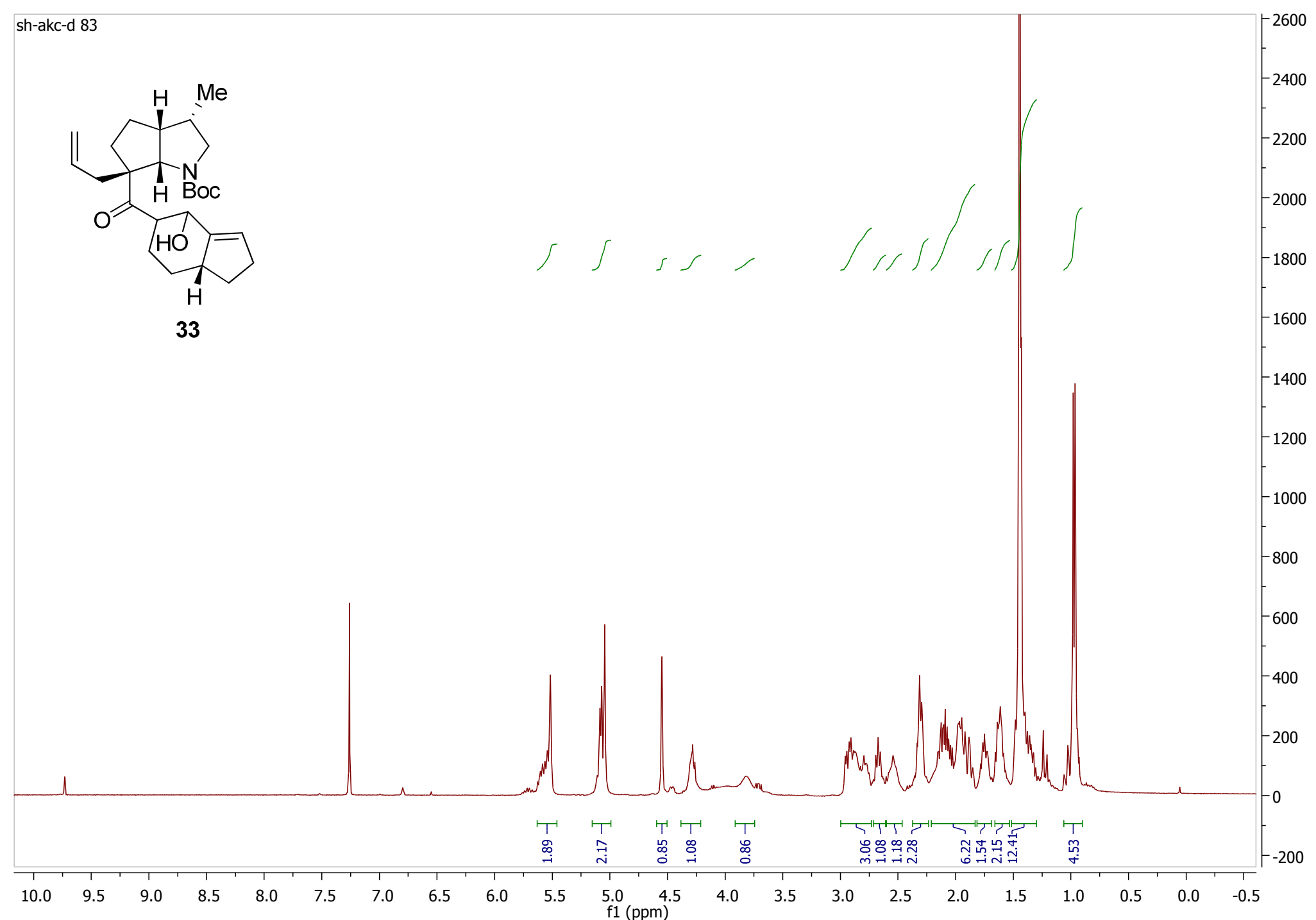

33, $\left({ }^{1} \mathrm{HNMR}, \mathrm{CDCl}_{3}, 400 \mathrm{MHz}\right)$ 


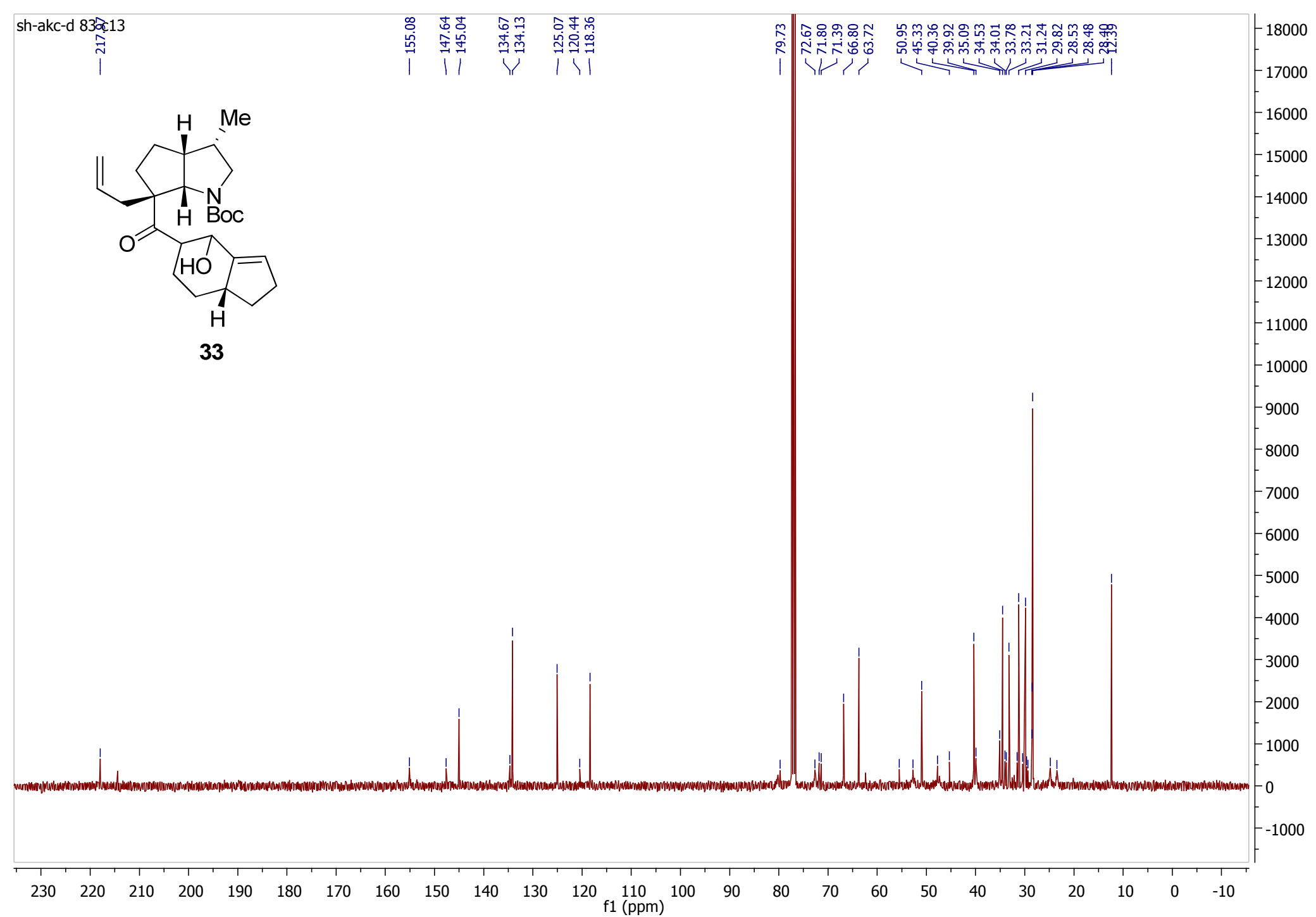

33, $\left({ }^{13} \mathrm{C} \mathrm{NMR}, \mathrm{CDCl}_{3}, 100 \mathrm{MHz}\right)$ 


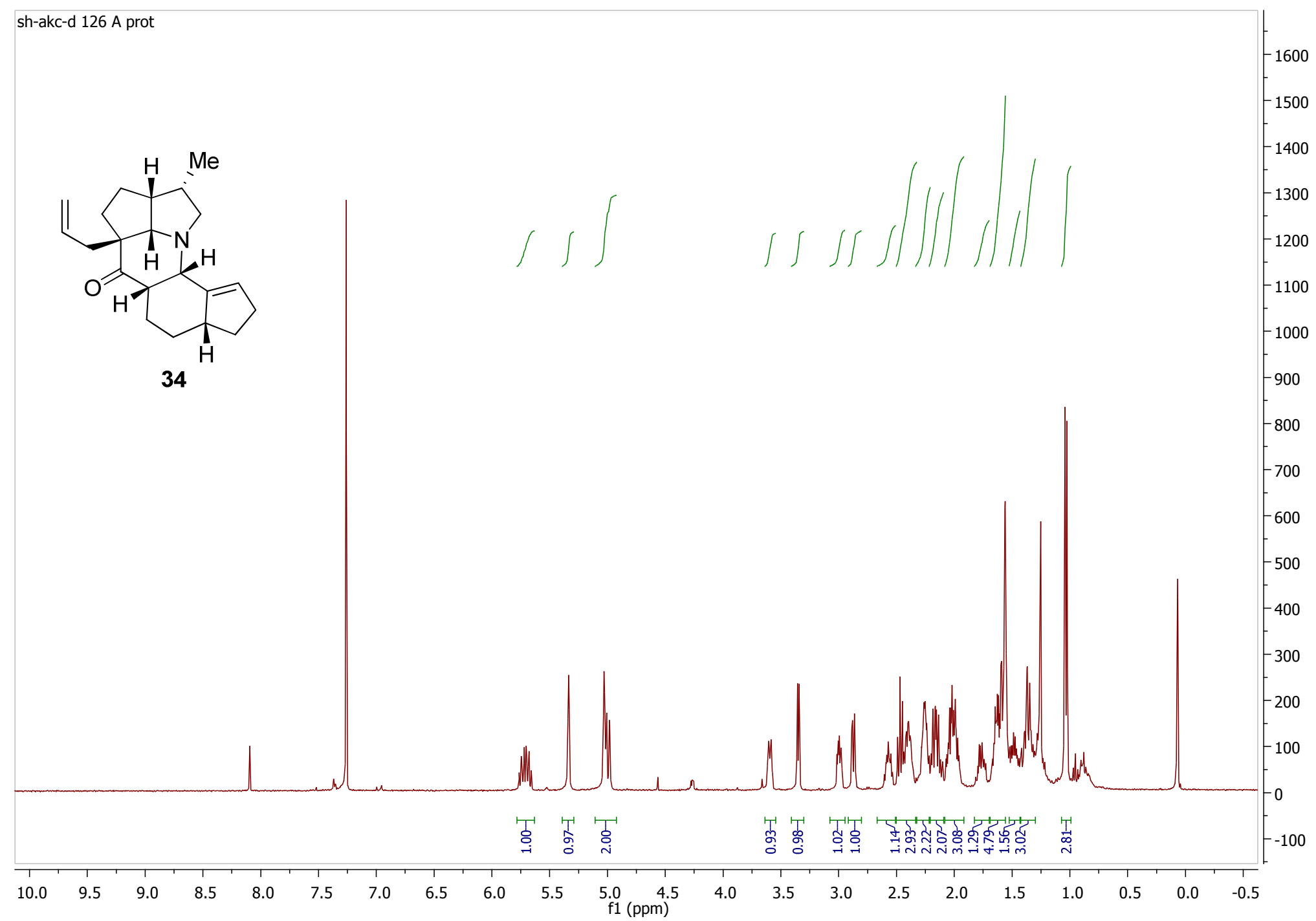

34, $\left({ }^{1} \mathrm{H} N M R, \mathrm{CDCl}_{3}, 400 \mathrm{MHz}\right)$. For DFT data, see S96. For X-ray data, see S137-138. 


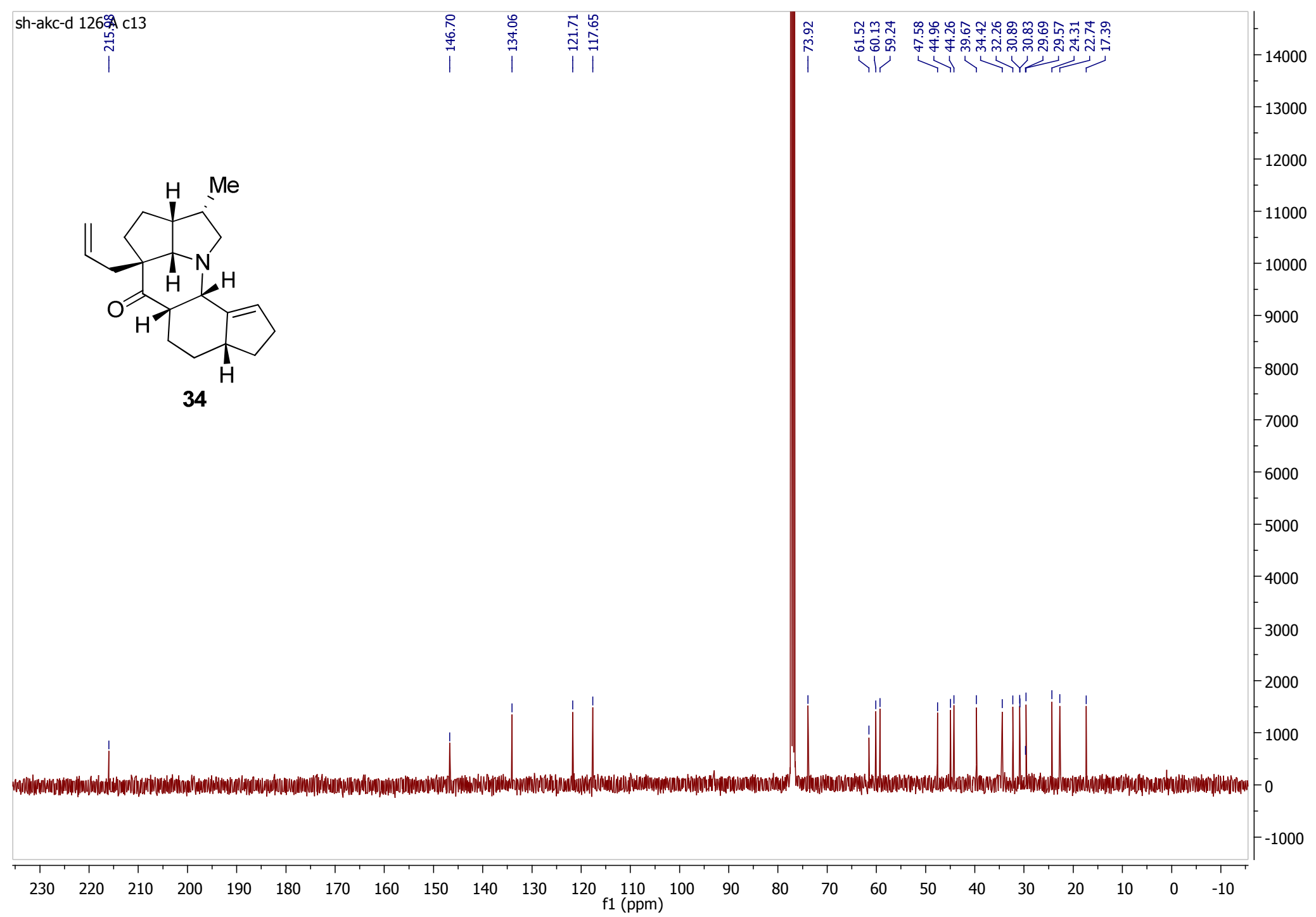

34, $\left({ }^{13} \mathrm{C} \mathrm{NMR}, \mathrm{CDCl}_{3}, 100 \mathrm{MHz}\right)$. For DFT data, see S96. For X-ray data, see S137-138. 


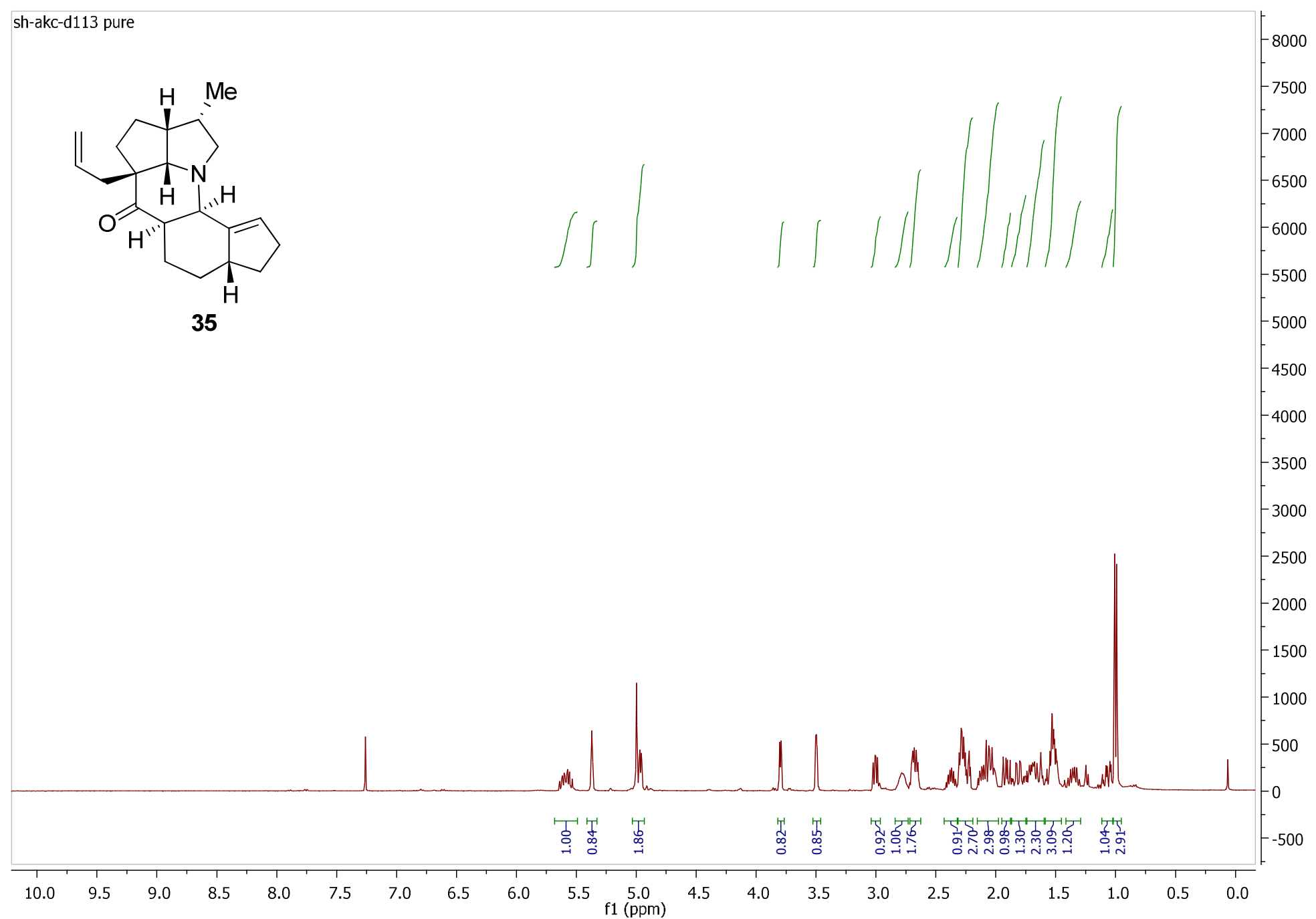

35, $\left({ }^{1} \mathrm{H} N M R, \mathrm{CDCl}_{3}, 400 \mathrm{MHz}\right)$. For DFT data, see S81 and S102. 


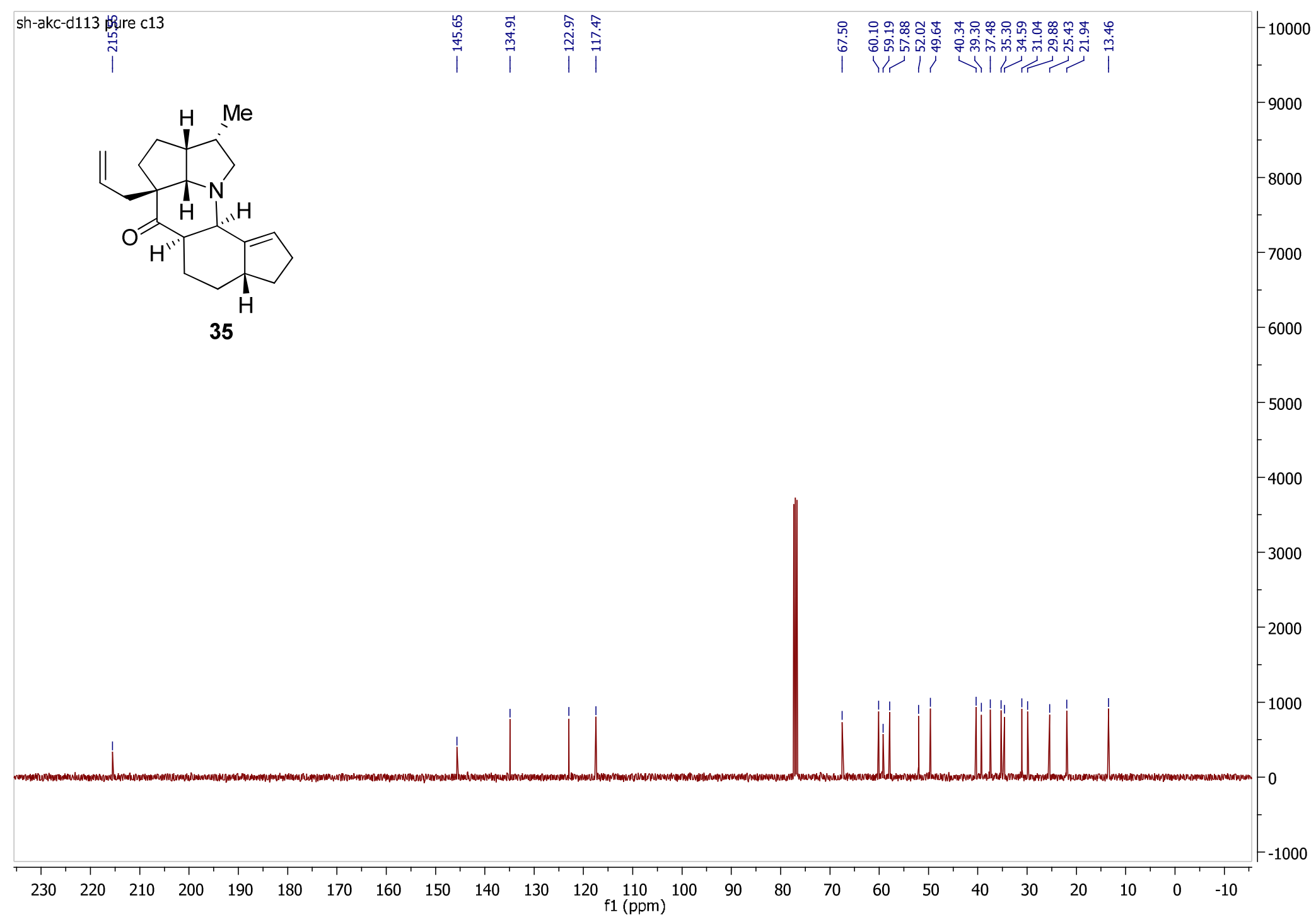

35, $\left({ }^{13} \mathrm{C} \mathrm{NMR}, \mathrm{CDCl}_{3}, 100 \mathrm{MHz}\right)$. For DFT data, see S81 and S102. 


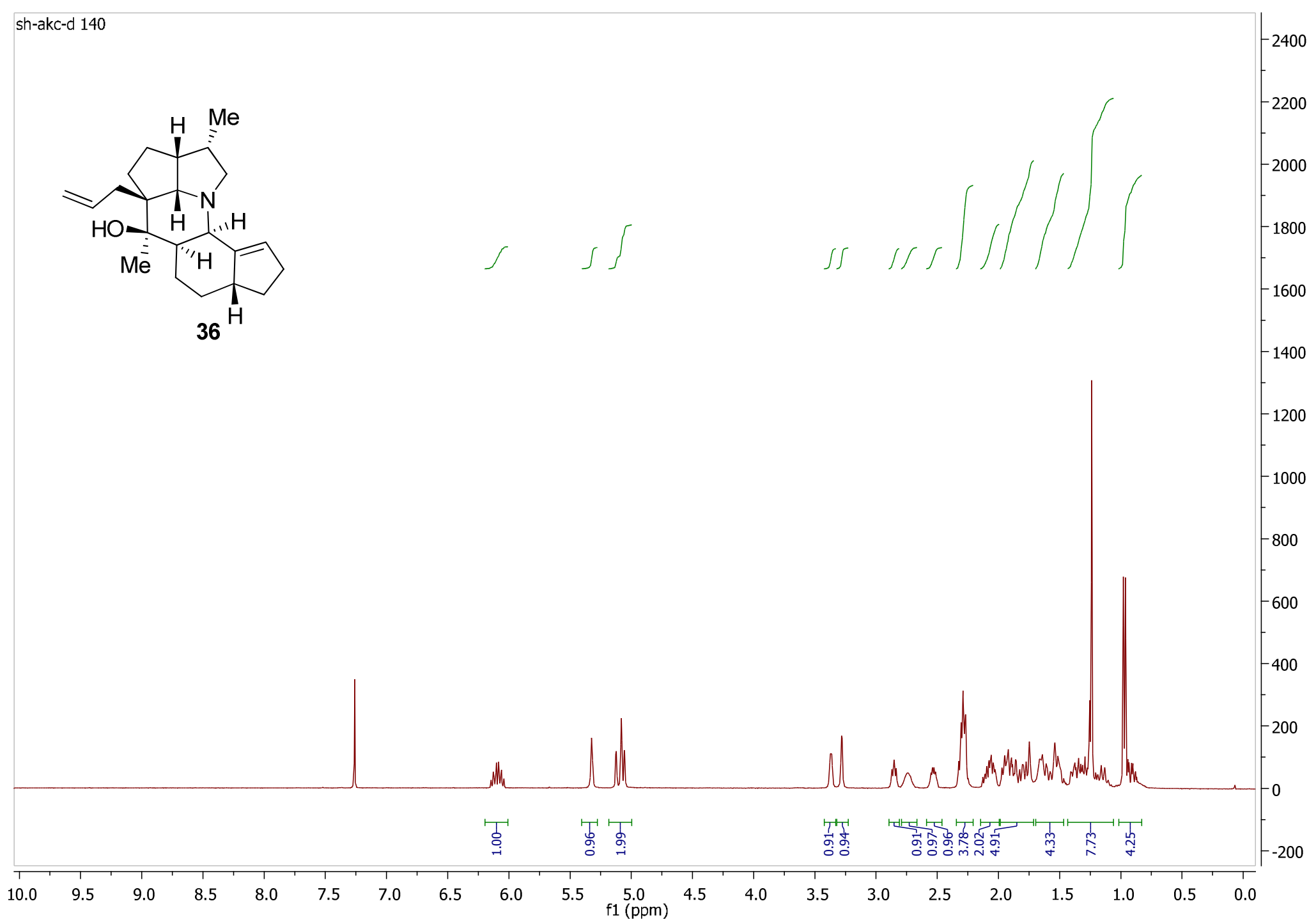

36, $\left({ }^{1} \mathrm{H} N M R, \mathrm{CDCl}_{3}, 400 \mathrm{MHz}\right)$ 


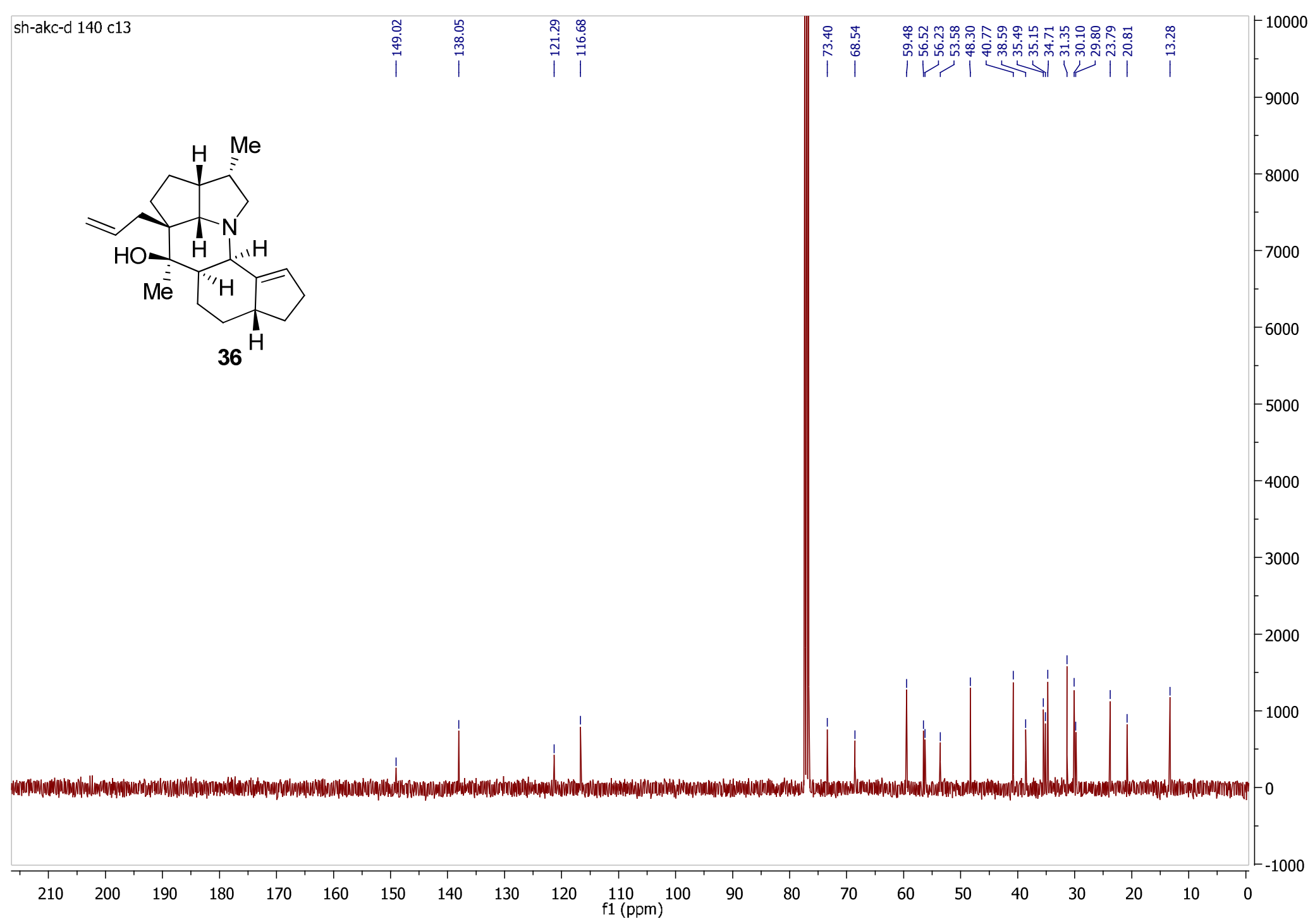

36, $\left({ }^{13} \mathrm{C} \mathrm{NMR}, \mathrm{CDCl}_{3}, 100 \mathrm{MHz}\right)$ 


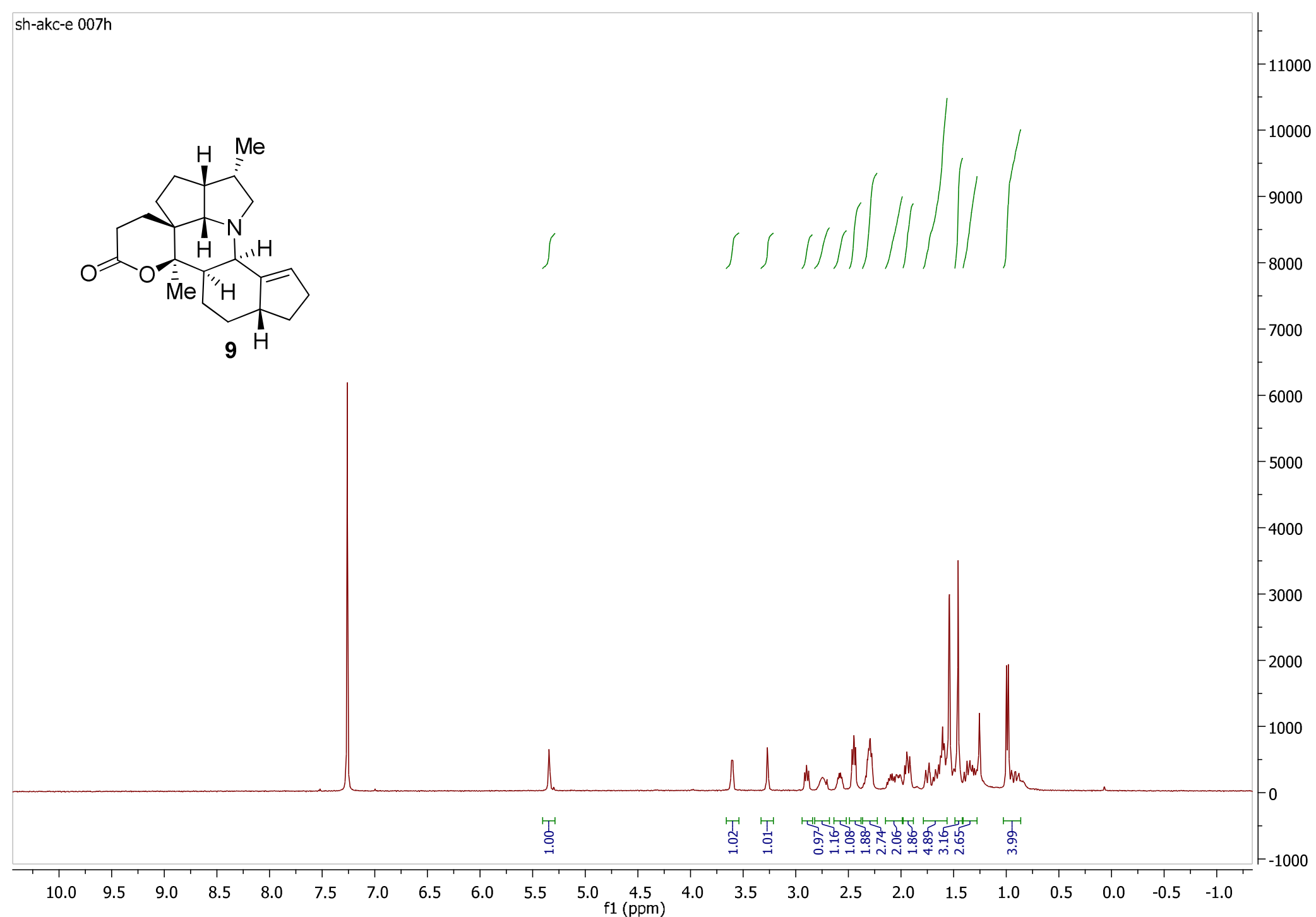

9, ( $\left.{ }^{1} \mathrm{H} N M R, \mathrm{CDCl}_{3}, 400 \mathrm{MHz}\right)$. For DFT data, see S122. For X-ray data, see S134. 


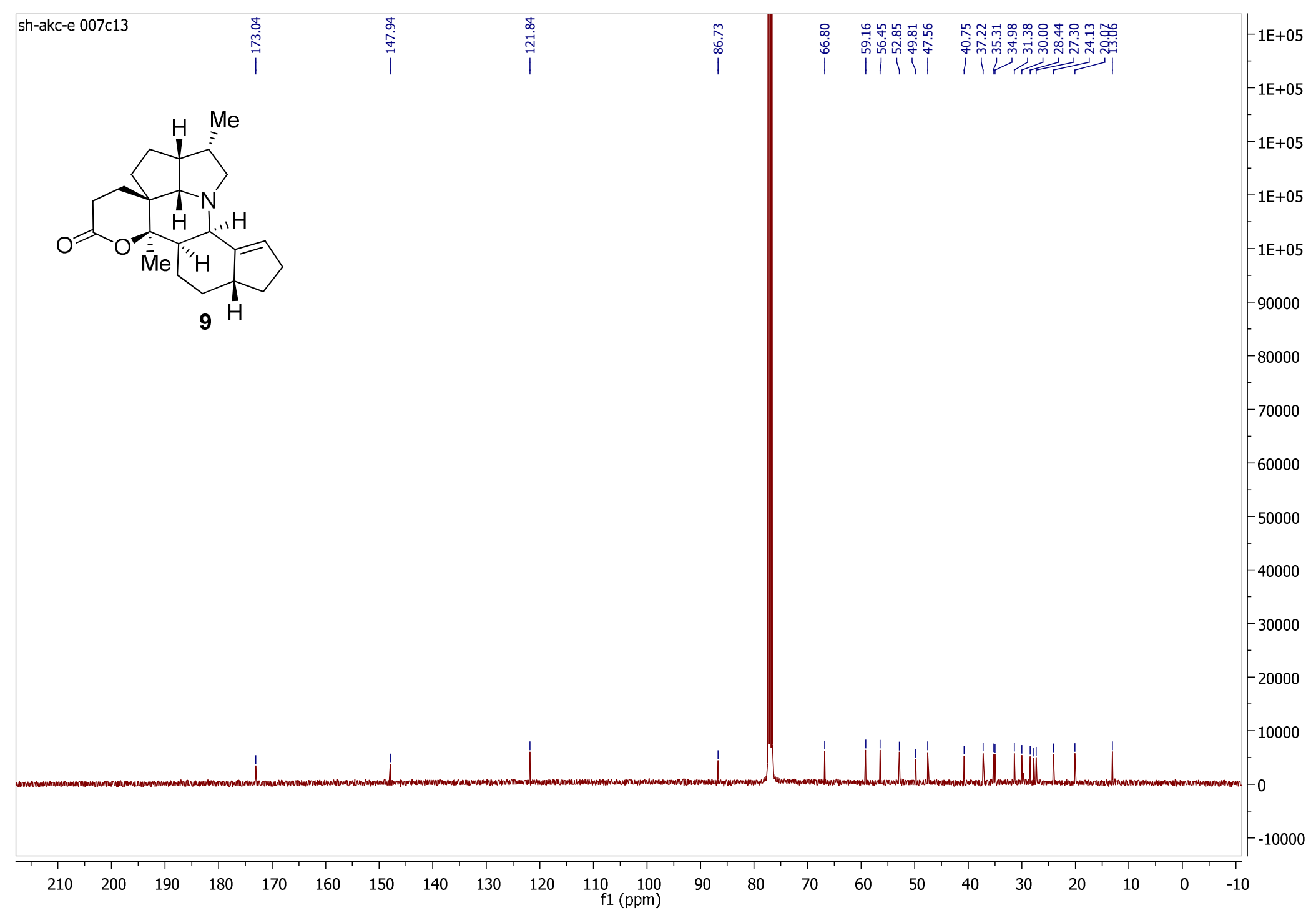

9, $\left({ }^{13} \mathrm{C} \mathrm{NMR}, \mathrm{CDCl}_{3}, 100 \mathrm{MHz}\right)$. For DFT data, see S122. For X-ray data, see S134. 


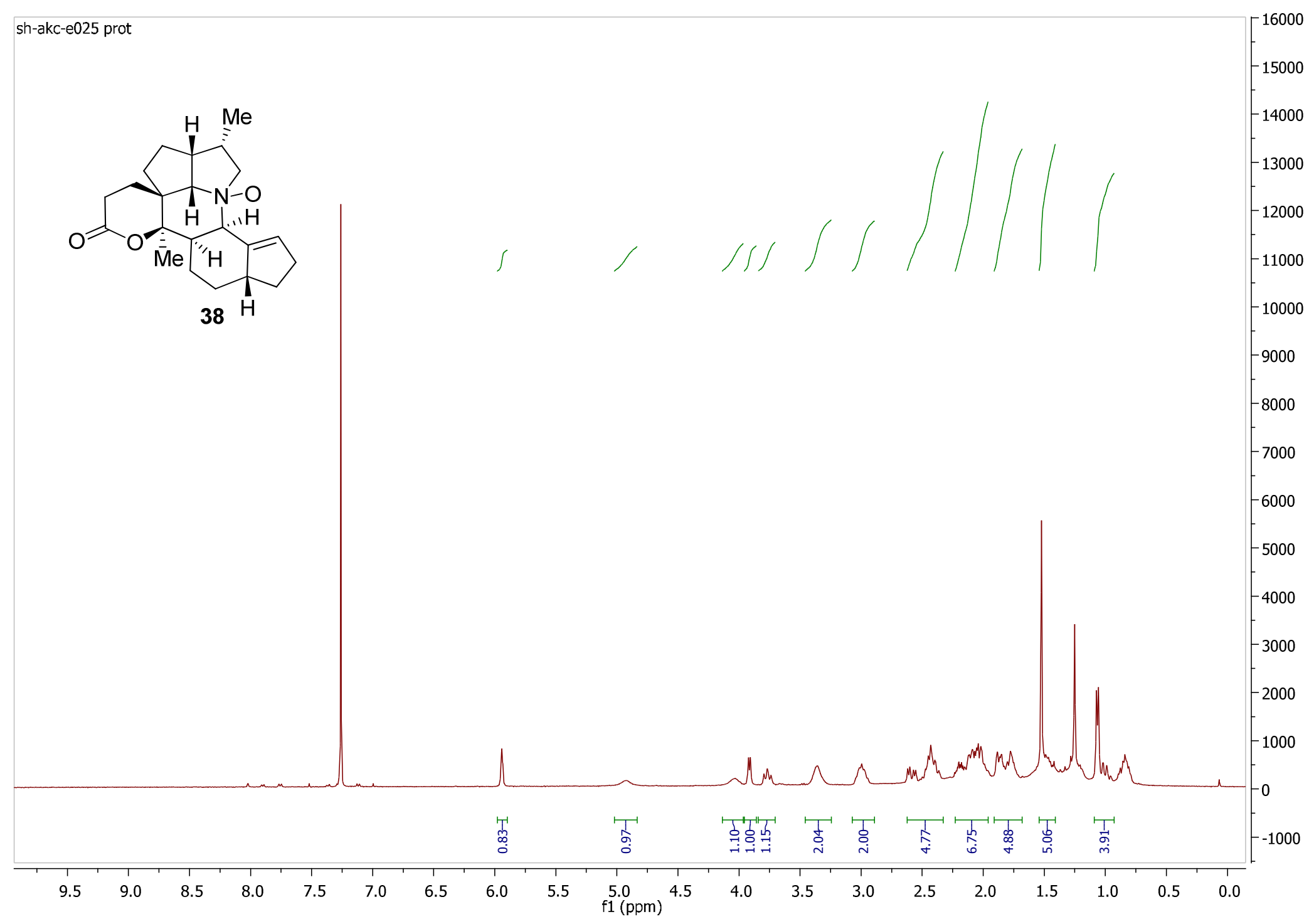

38, $\left({ }^{1} \mathrm{H} N M R, \mathrm{CDCl}_{3}, 400 \mathrm{MHz}\right)$ 


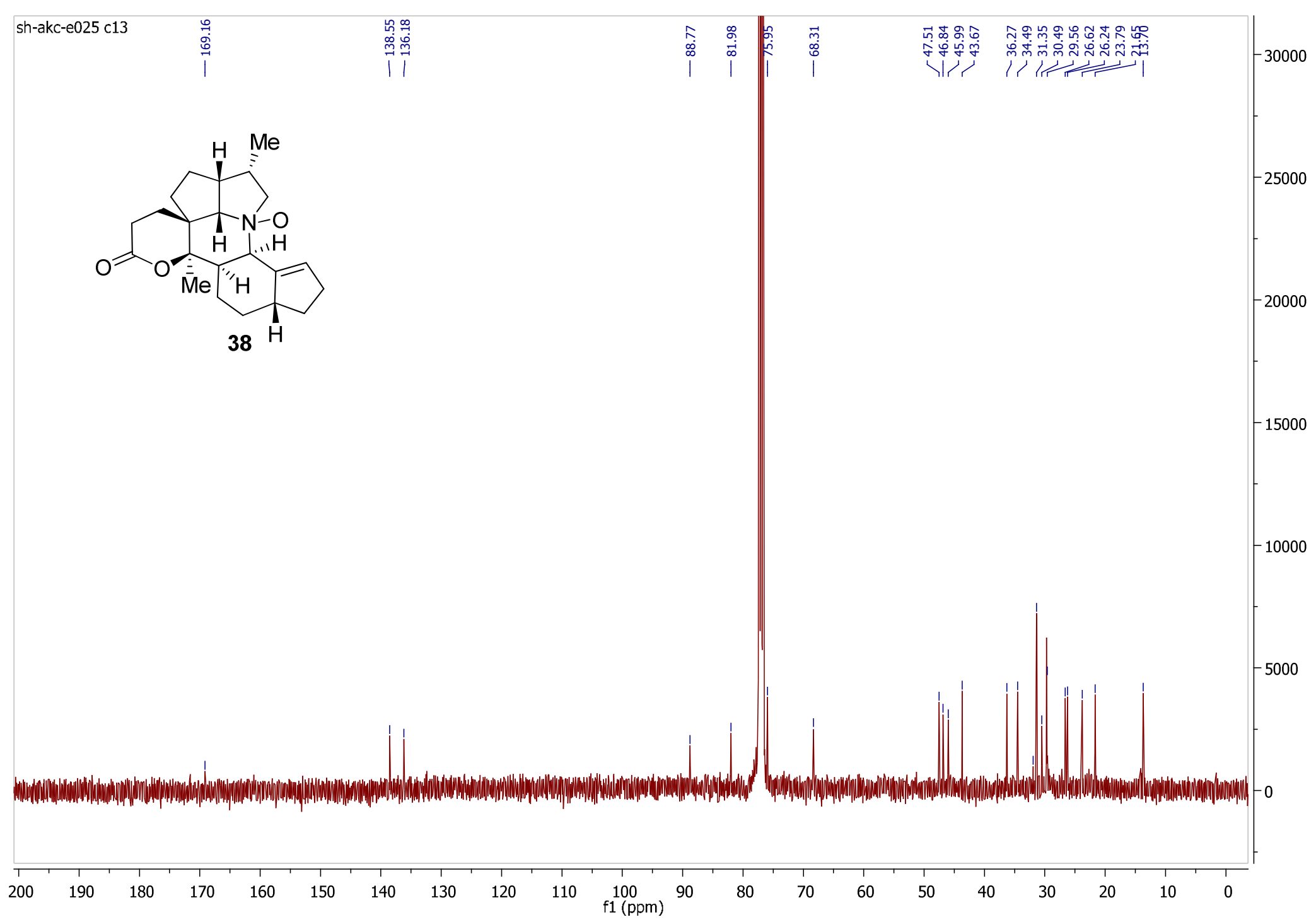

38, $\left({ }^{13} \mathrm{C} \mathrm{NMR}, \mathrm{CDCl}_{3}, 100 \mathrm{MHz}\right)$ 


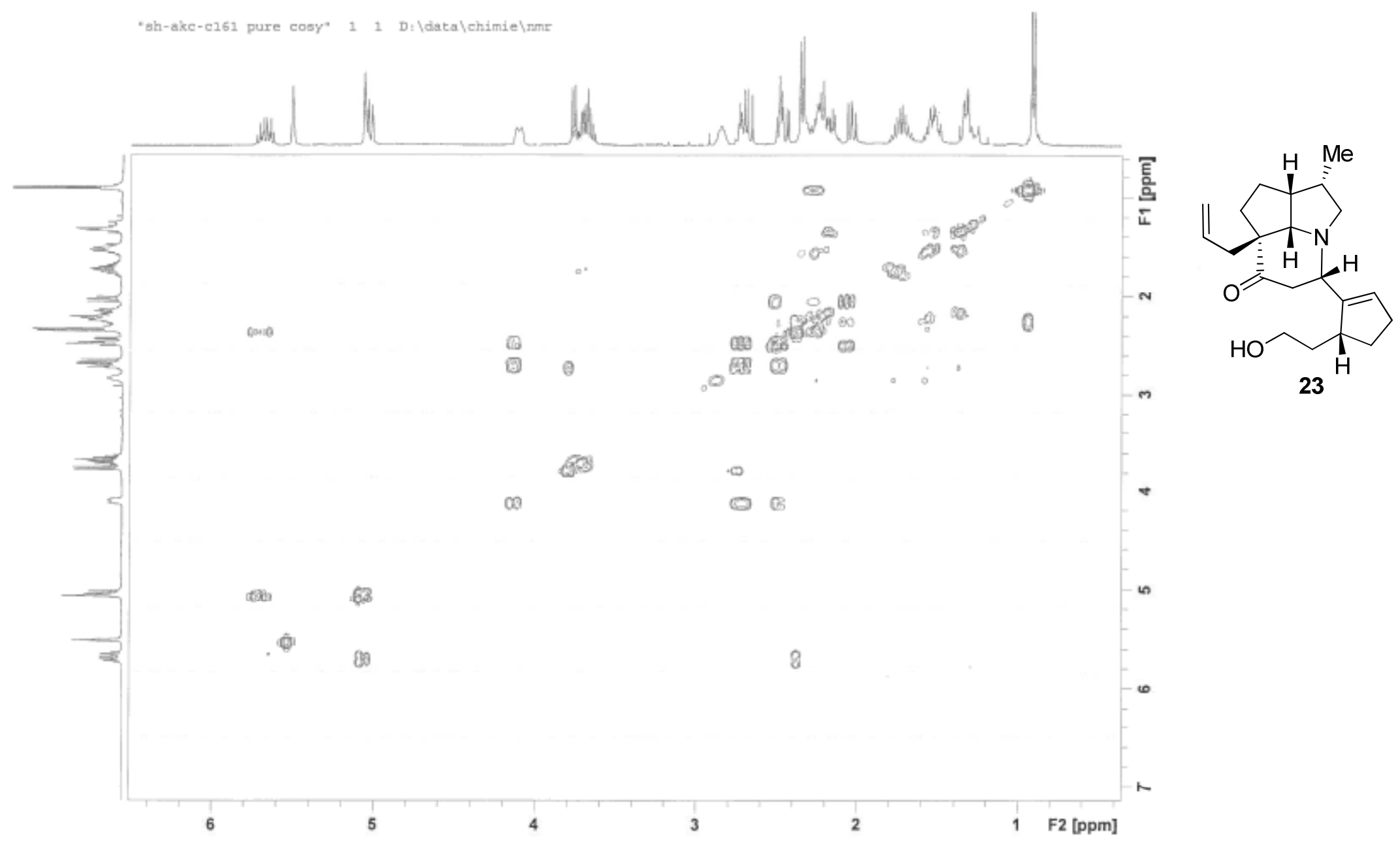




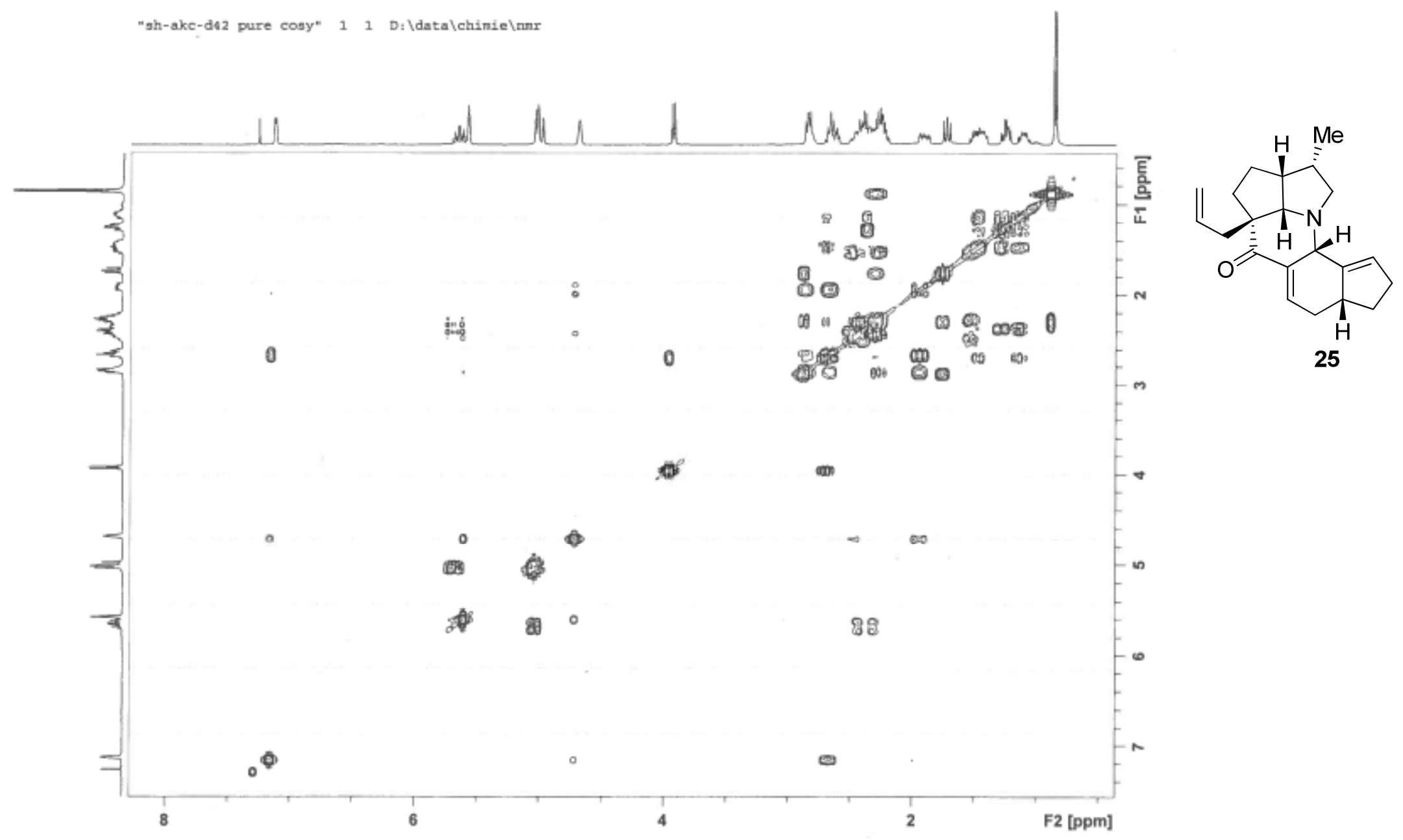




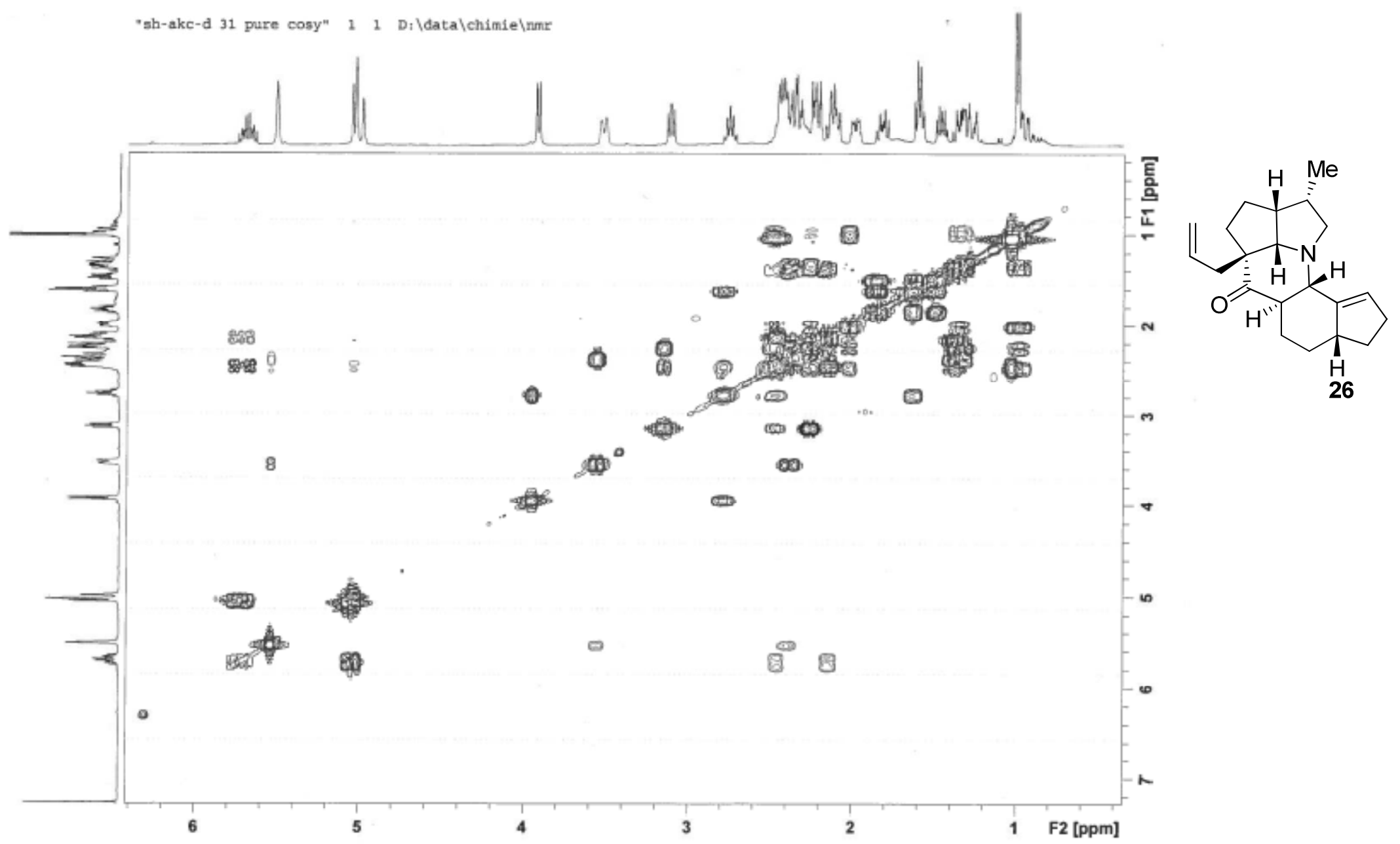




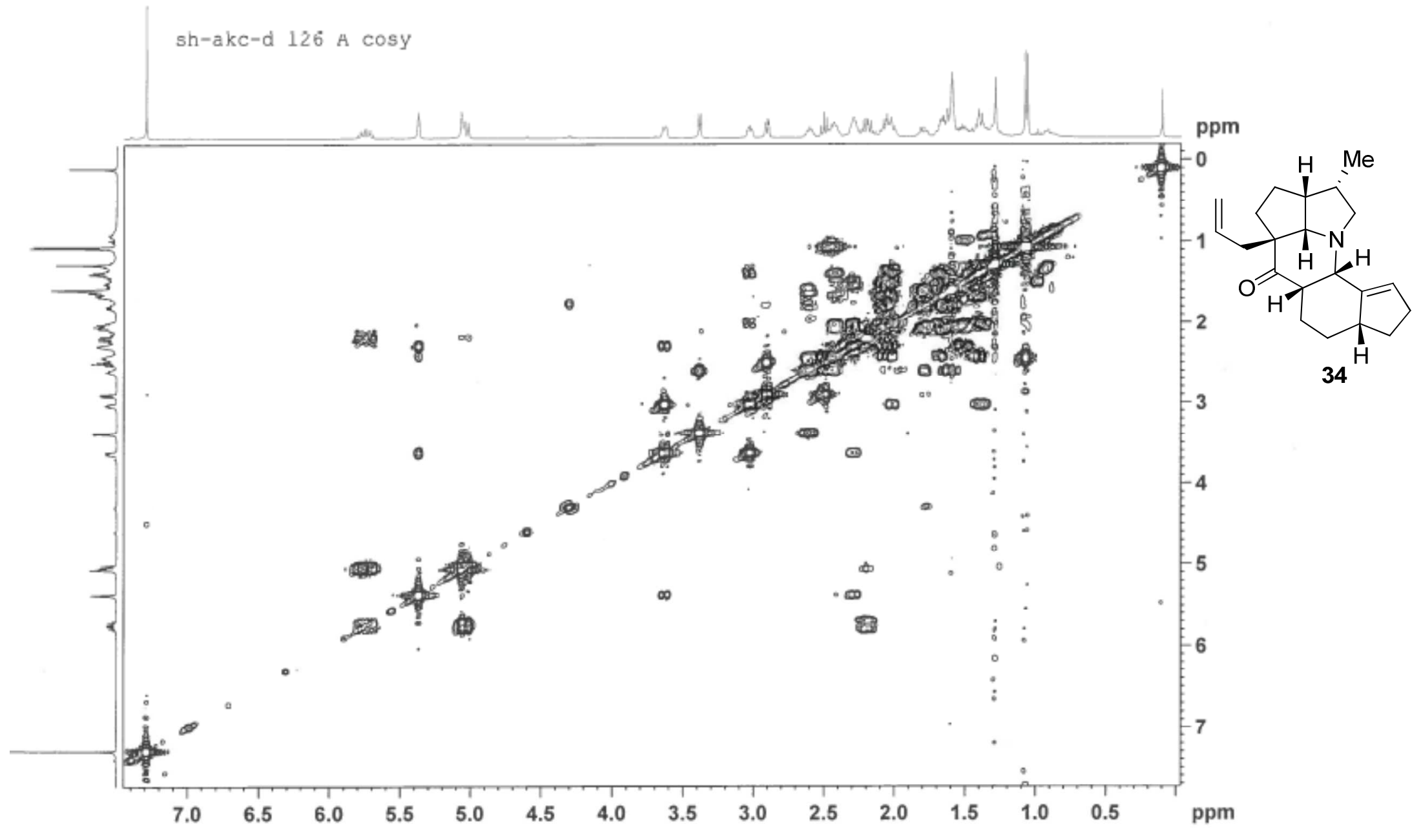




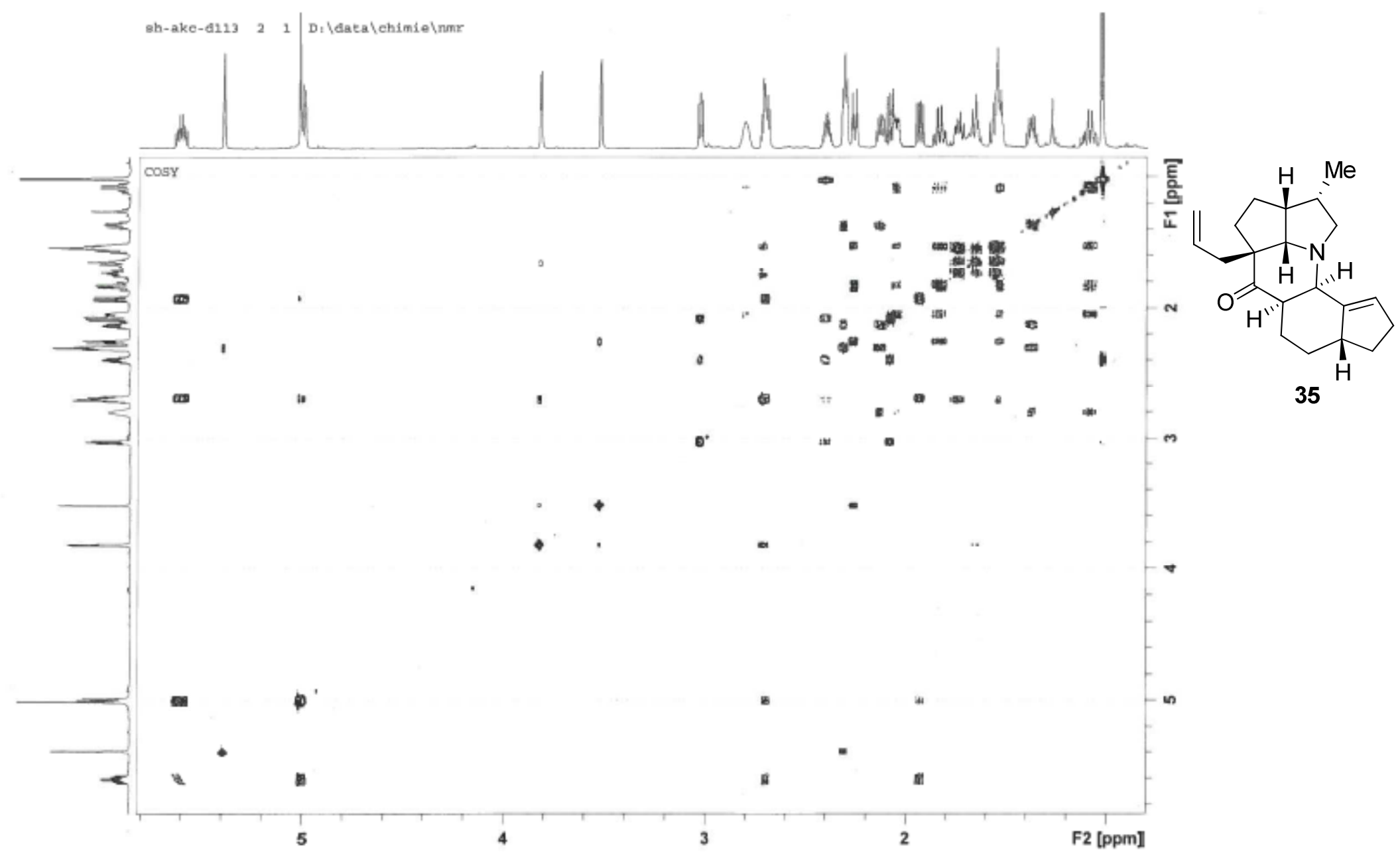




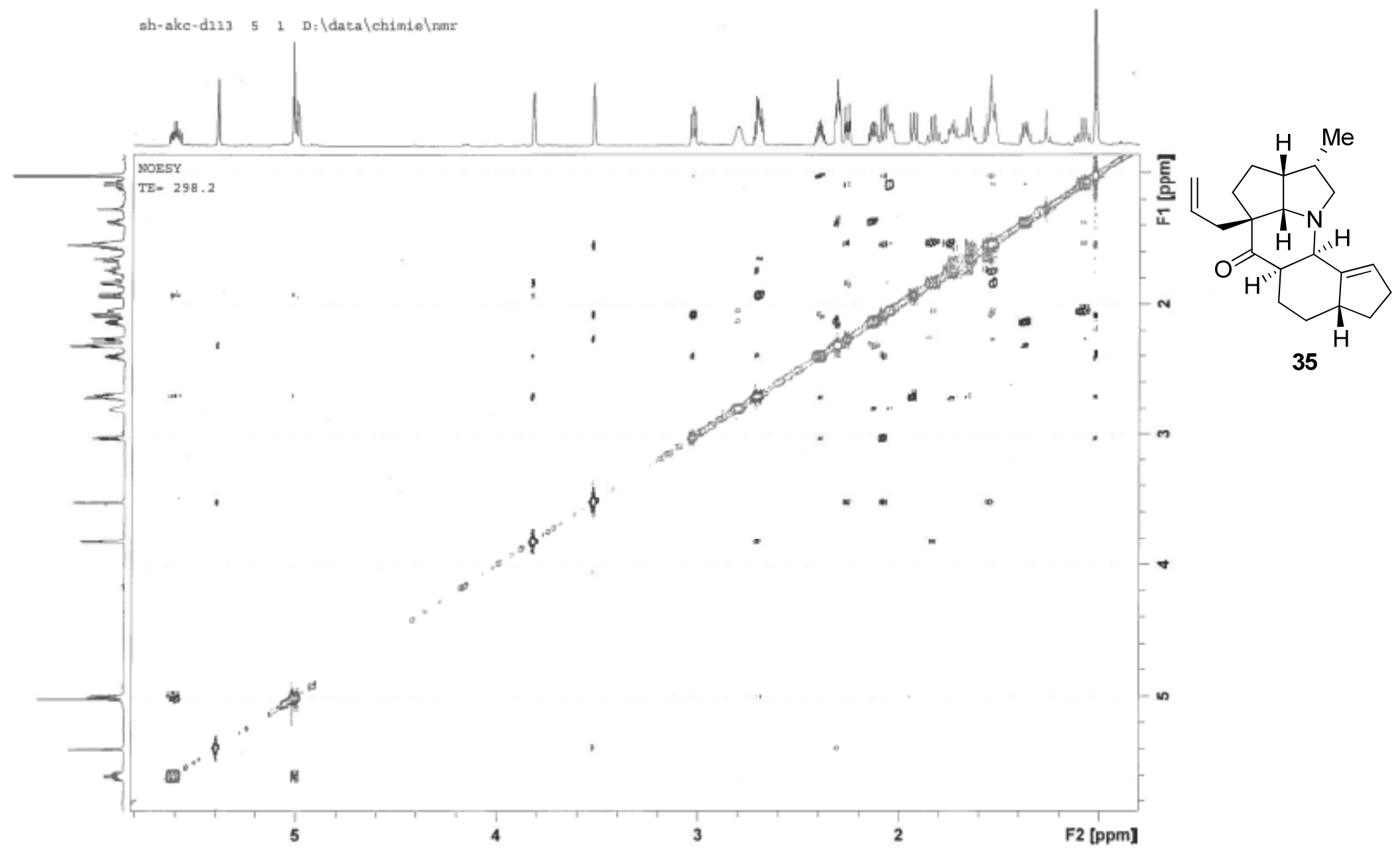




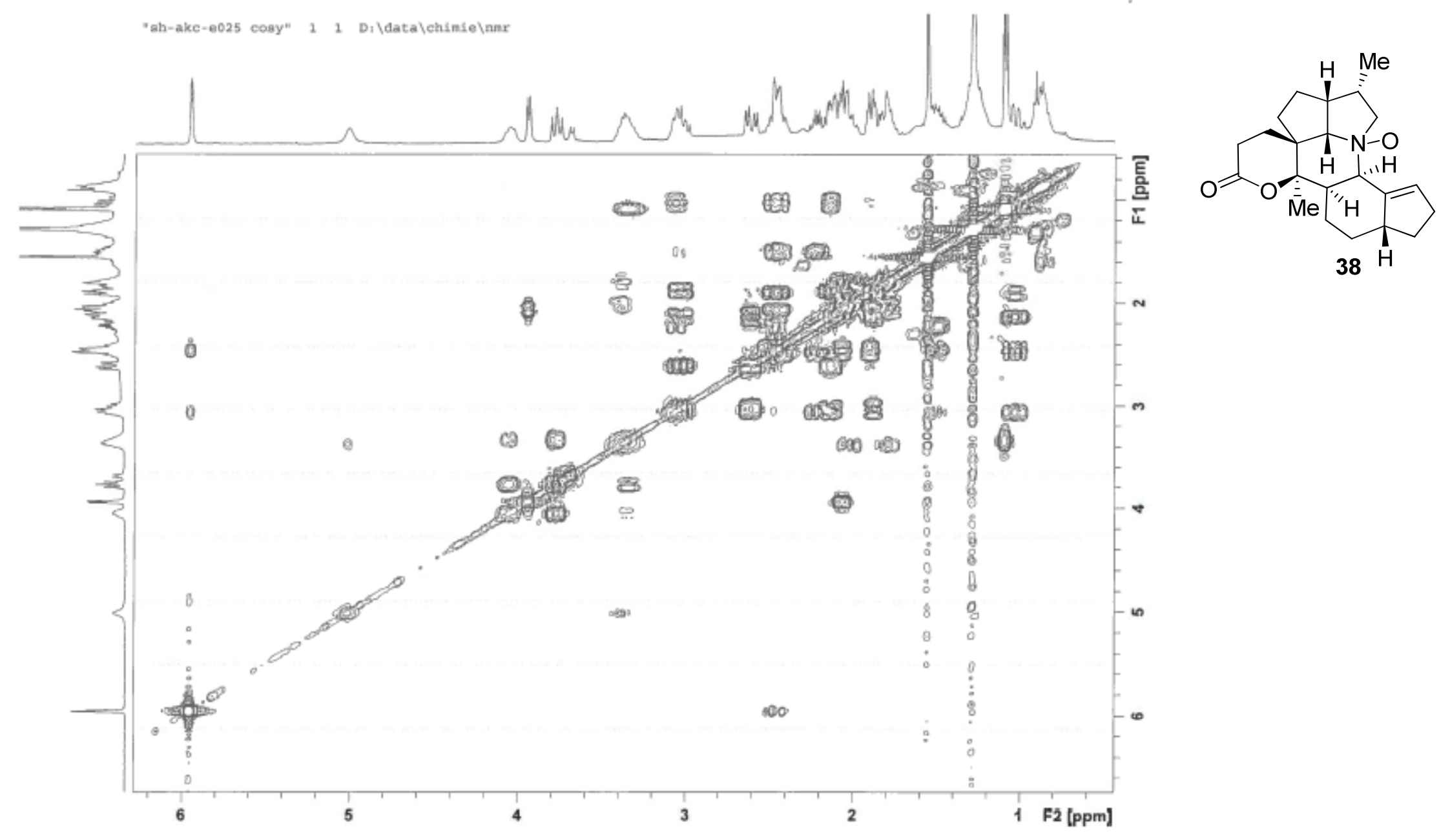




\section{DFT data (Computational Details)}

\subsection{DFT Methods}

All quantum mechanical calculations have been achieved using the Gaussian09 rev D.01 software package. Geometries of all the investigated systems were fully optimized at the spin-restricted density functional theory level using the dispersion-corrected $\omega B$ B7x-D exchange-correlation functional. ${ }^{1}$ The balanced polarized triple-zeta basis set def2-TZVP from Ahlrichs and co-workers ${ }^{2,3}$ have been used for all atoms. Potential energy surface minima found upon optimization were confirmed by frequency calculations and free energies were corrected to account for the zero-point energy. Optimized geometries were verified as minima (i.e. zero imaginary frequencies). The Synchronous Transit-Guided QuasiNewton (STQN) method ${ }^{4,5}$ was used for locating transition structures. These structures were further verified as saddle points by frequency calculations (i.e. one and only one imaginary frequency). The bulk solvent effects have been included through the Integral Equation Formalism version of the Polarizable Continuum Model (IEF-PCM). ${ }^{6}$

Total DFT energy, RMSD at the end of the geometry optimization, number of imaginary frequencies and Cartesian coordinates are given in the next section for all calculated structures.

Gaussian full reference. Gaussian09, Revision D.01, M. J. Frisch, G. W. Trucks, H. B. Schlegel, G. E. Scuseria, M. A. Robb, J. R. Cheeseman, G. Scalmani, V. Barone, B. Mennucci, G. A. Petersson, H. Nakatsuji, M. Caricato, X. Li, H. P. Hratchian, A. F. Izmaylov, J. Bloino, G. Zheng, J. L. Sonnenberg, M. Hada, M. Ehara, K. Toyota, R. Fukuda, J. Hasegawa, M. Ishida, T. Nakajima, Y. Honda, O. Kitao, H. Nakai, T. Vreven, J. A. Montgomery, Jr., J. E. Peralta, F. Ogliaro, M. Bearpark, J. J. Heyd, E. Brothers, K. N. Kudin, V. N. Staroverov, T. Keith, R. Kobayashi, J. Normand, K. Raghavachari, A. Rendell, J. C. Burant, S. S. Iyengar, J. Tomasi, M. Cossi, N. Rega, J. M. Millam, M. Klene, J. E. Knox, J. B. Cross, V. Bakken, C. Adamo, J. Jaramillo, R. Gomperts, R. E. Stratmann, O. Yazyev, A. J. Austin, R. Cammi, C. Pomelli, J. W. Ochterski, R. L. Martin, K. Morokuma, V. G. Zakrzewski, G. A. Voth, P. Salvador, J. J. Dannenberg, S. Dapprich, A. D. Daniels, O. Farkas, J. B. Foresman, J. V. Ortiz, J. Cioslowskiand, D. J. Fox, Gaussian, Inc., Wallingford CT, 2010. 
3.2 Alignment of DFT and XRD structures
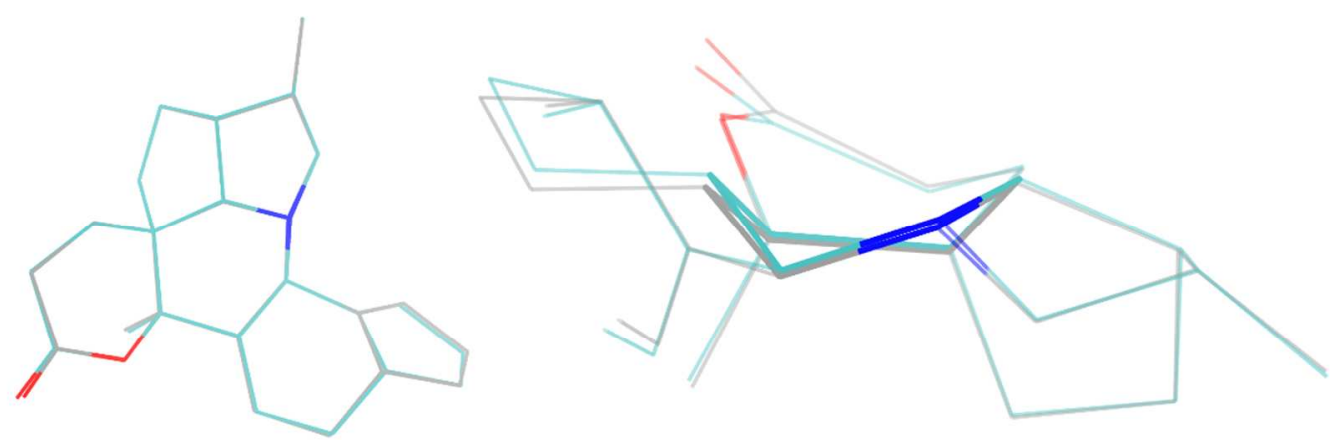

Figure S1. Deoxycalyciphylline B: XRD (cyan) vs DFT (grey). RMSD between the two geometries $=0.11 \AA$

Figure S2. 5-iso-Daphlongamine H: XRD (cyan) vs DFT (grey). RMSD between the two geometries $=0.08 \AA$
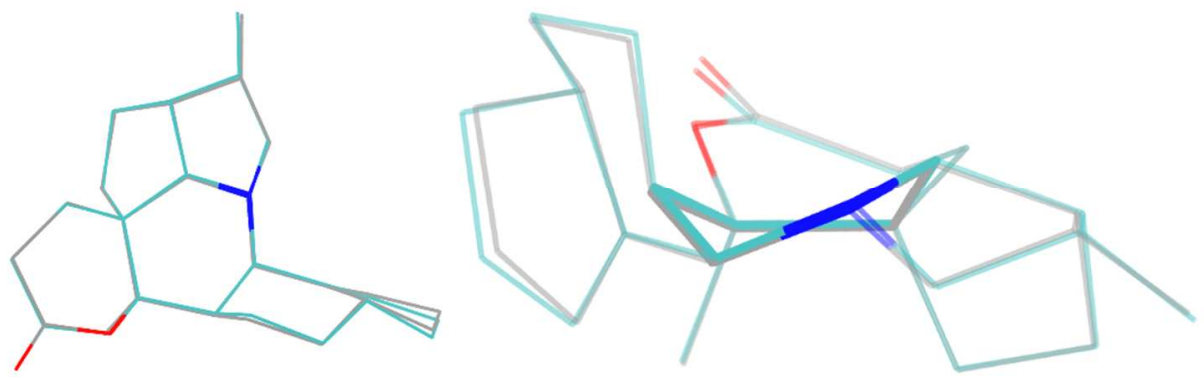

S70 
3.3 $C$ - and $O$-quenching of the Li-enolate
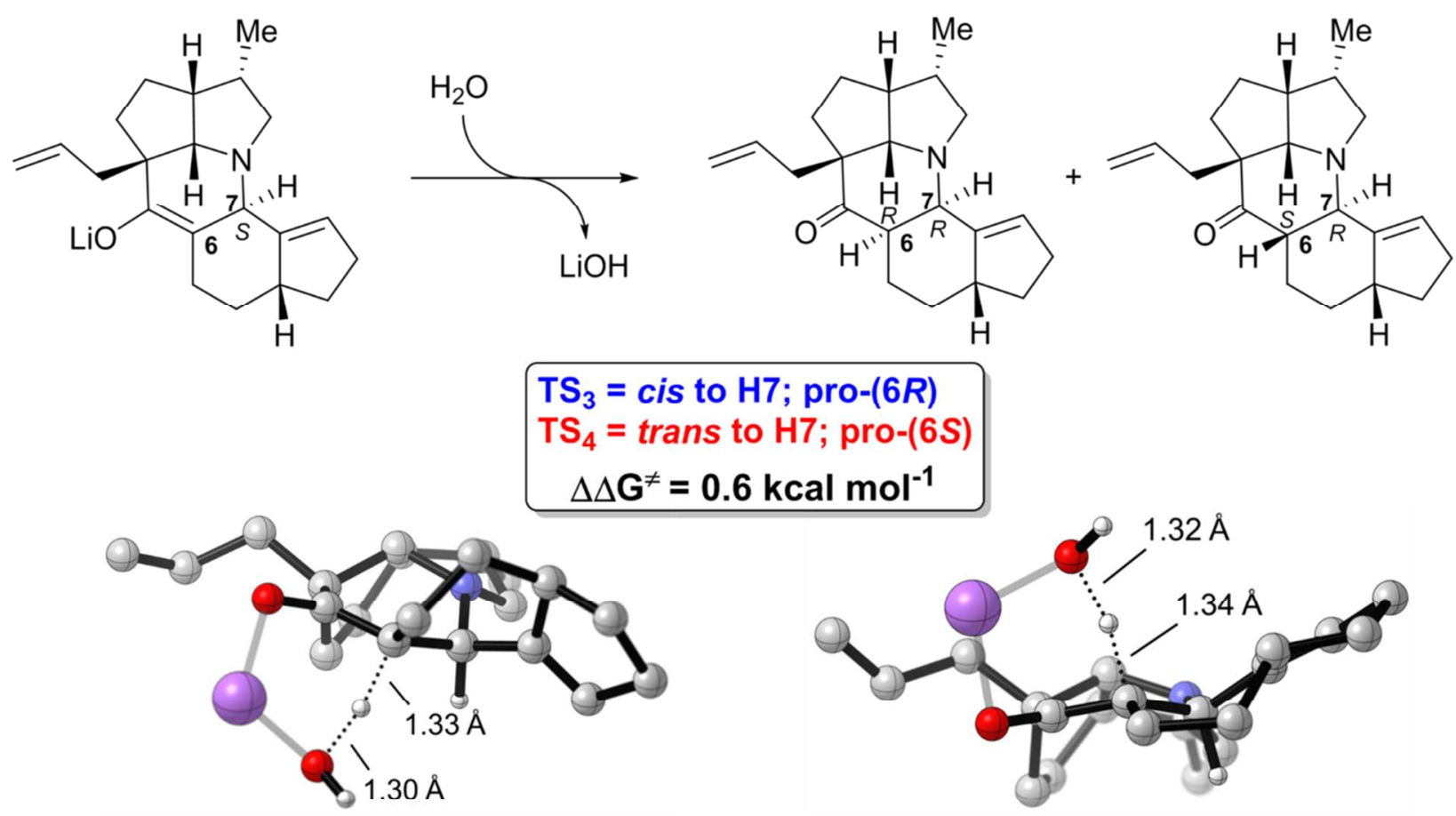

$\mathrm{TS}_{3} \equiv 0 \mathrm{kcal} \mathrm{mol}^{-1}$

$$
\mathrm{TS}_{4}=0.6 \mathrm{kcal} \mathrm{mol}^{-1}
$$

Figure S3. Transition states for the aqueous quenching (IEFPCM) of the Li-enolate to the enol carbon from both faces ( $C$-quenching).

Conventional methods systematically failed to locate the $O$-quenching saddle point, highlighting a relatively flat potential-energy landscape. The $O$-quenching transition state $\mathrm{TS}_{5}$ was therefore located by performing a rigid potential energy surface (PES) scan, varying two internal coordinates (the $\mathrm{H}-\mathrm{O}-\mathrm{Li}$ bend angle and the $\mathrm{O}-\mathrm{H}$ distance, as values labeled in Figure $\mathrm{B}$ ). This procedure showed two minima corresponding to the two bonded state of the water proton: an absolute minimum for the enolate- $\mathrm{Li}_{-} \mathrm{H}_{2} \mathrm{O}$ complex and a local minimum for the LiOH-enol complex. The saddle point connecting these two valleys then represents the transition state for the $O$-quenching and is 
found around $2 \mathrm{kcal} \mathrm{mol}^{-1}$.

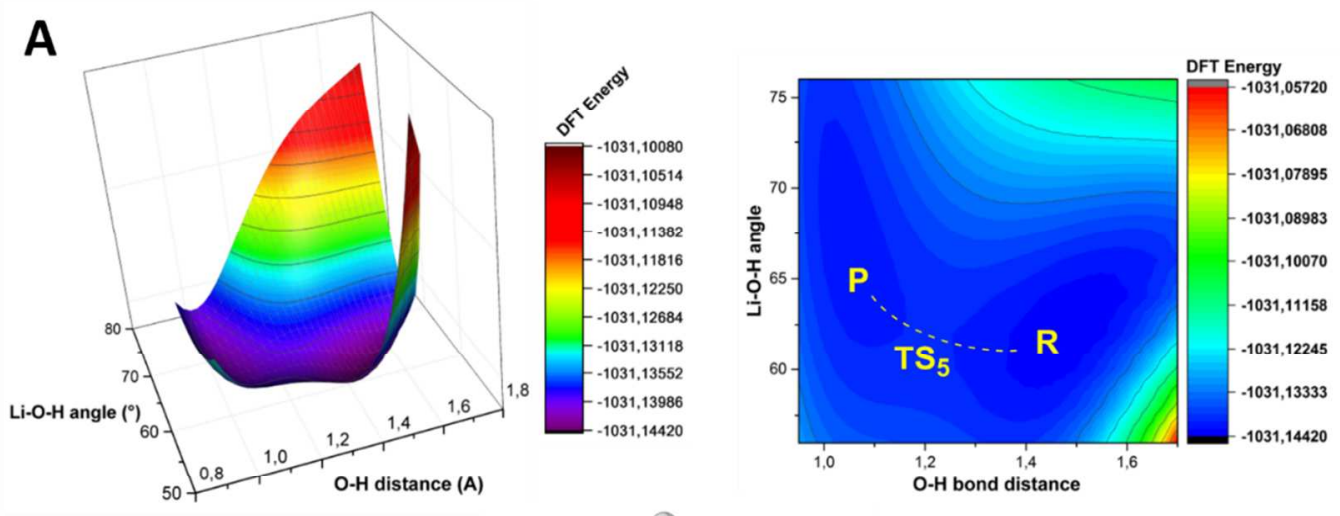

B
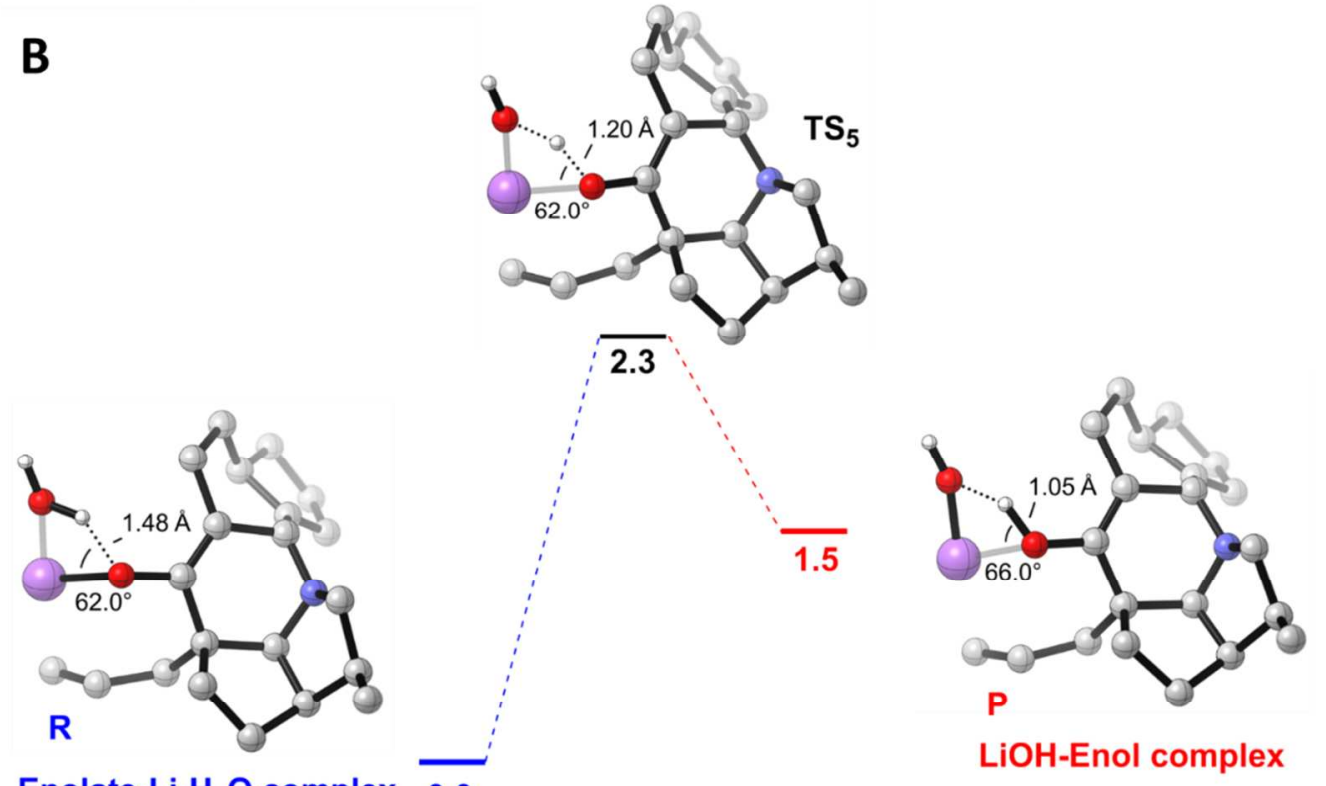

Enolate-Li- $\mathrm{H}_{2} \mathrm{O}$ complex $\overline{\mathbf{0 . 0}}$

Figure S4. PES scan for the proton transfer from water to the Li-enolate oxygen $(A)$ and the corresponding energy profile along the reaction coordinate (B). DFT energy in atomic units. 


\subsection{Geometries and optimization data}

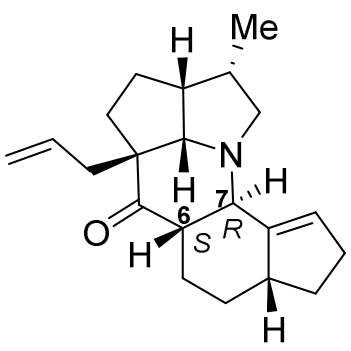

Level of theory: $\omega B$ 97x-D/def2TZVP

Total Energy $=-947.69583343 \mathrm{au}$

RMS Gradient Norm $=0.00000573 \mathrm{au}$

Imaginary Frequencies $=0$

Dipole Moment $=2.23$ Debye

\begin{tabular}{lrrc} 
Symbol & \multicolumn{1}{c}{$\mathbf{X}$} & \multicolumn{1}{c}{$\mathbf{Y}$} & $\mathbf{Z}$ \\
\hline $\mathrm{C}$ & -0.09347 & 2.421945 & 0.455906 \\
$\mathrm{H}$ & 0.096173 & 2.343284 & 1.541457 \\
$\mathrm{H}$ & -1.01917 & 2.992174 & 0.338414 \\
$\mathrm{C}$ & 0.822246 & -1.23519 & 0.804507 \\
$\mathrm{C}$ & -3.0091 & -1.81374 & 1.116906 \\
$\mathrm{H}$ & -3.2474 & -1.17234 & 1.971018 \\
$\mathrm{H}$ & -3.63042 & -2.70593 & 1.215349 \\
$\mathrm{C}$ & 1.770899 & -0.55655 & -0.17443 \\
$\mathrm{C}$ & 1.686184 & 4.265259 & 0.487138 \\
$\mathrm{H}$ & 2.03699 & 3.979005 & 1.480538 \\
$\mathrm{H}$ & 0.950067 & 5.062024 & 0.611155 \\
$\mathrm{H}$ & 2.53437 & 4.678505 & -0.06203 \\
$\mathrm{C}$ & 1.07682 & 0.714046 & -0.77796 \\
$\mathrm{H}$ & 0.943279 & 0.566467 & -1.85617 \\
$\mathrm{C}$ & -1.13106 & 0.185684 & 0.372217 \\
$\mathrm{H}$ & -1.26853 & 0.42388 & 1.441323 \\
$\mathrm{C}$ & 3.052139 & -0.045 & 0.503956 \\
$\mathrm{H}$ & 3.285097 & -0.61472 & 1.401937
\end{tabular}




$\begin{array}{lrrr}\text { H } & 3.892068 & -0.16234 & -0.18551 \\ \mathrm{C} & 1.08339 & 3.082723 & -0.25056 \\ \mathrm{H} & 0.73328 & 3.419109 & -1.23127 \\ \mathrm{C} & -3.1637 & 1.180704 & -0.85447 \\ \mathrm{H} & -2.76189 & 2.158872 & -1.08401 \\ \mathrm{C} & 2.798656 & 1.431556 & 0.765314 \\ \mathrm{H} & 2.188611 & 1.548333 & 1.665774 \\ \mathrm{H} & 3.716344 & 1.999351 & 0.921038 \\ \mathrm{C} & -2.5016 & 0.205116 & -0.24916 \\ \mathrm{C} & -3.33389 & -1.05365 & -0.16008 \\ \mathrm{H} & -3.07074 & -1.69791 & -1.01262 \\ \mathrm{C} & -1.51799 & -2.2075 & 1.147961 \\ \mathrm{H} & -1.15385 & -2.23555 & 2.175267 \\ \mathrm{H} & -1.39437 & -3.21775 & 0.752187 \\ \mathrm{C} & 4.077858 & -3.10125 & -1.25174 \\ \mathrm{H} & 4.605611 & -2.50732 & -1.99074 \\ \mathrm{H} & 4.583958 & -3.98092 & -0.8747 \\ \mathrm{C} & 2.107235 & -1.56661 & -1.30026 \\ \mathrm{H} & 1.183109 & -1.89061 & -1.78902 \\ \mathrm{H} & 2.688671 & -1.03355 & -2.05745 \\ \mathrm{C} & 2.868959 & -2.76547 & -0.83037 \\ \mathrm{H} & 2.381788 & -3.38255 & -0.08024 \\ \mathrm{C} & -4.75444 & -0.51883 & -0.36053 \\ \mathrm{H} & -5.43072 & -1.24762 & -0.80889 \\ \mathrm{H} & -5.16708 & -0.23448 & 0.611979 \\ \mathrm{C} & -4.56194 & 0.746386 & -1.22035 \\ \mathrm{H} & -4.63361 & 0.516266 & -2.28984 \\ \mathrm{H} & -5.31208 & 1.512406 & -1.01451 \\ \mathrm{C} & -0.6173 & -1.26733 & 0.337624 \\ \mathrm{H} & -0.6113 & -1.57083 & -0.7137 \\ \mathrm{C} & 2.019398 & 1.892163 & -0.4703\end{array}$




$\begin{array}{lrrr}\mathrm{H} & 2.714745 & 2.068222 & -1.2944 \\ \mathrm{~N} & -0.19025 & 1.126969 & -0.17882 \\ \mathrm{O} & 1.17788 & -1.68364 & 1.865197\end{array}$

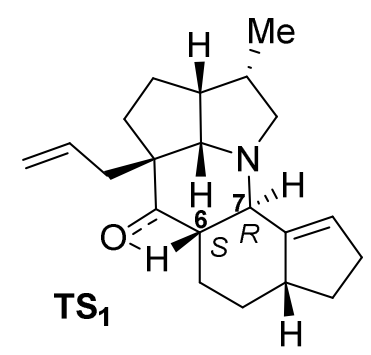

Level of theory: $\omega B 97 x-D /$ def2TZVP

Total Energy $=-947.57659354 \mathrm{au}$

RMS Gradient Norm $=0.00000405$ au

Imaginary Frequencies $=1$

Dipole Moment $=2.74$ Debye

\begin{tabular}{lrrr} 
Symbol & $\mathbf{X}$ & $\mathbf{Y}$ & $\mathbf{Z}$ \\
\hline $\mathrm{C}$ & -0.54268 & 2.245011 & -1.1088 \\
$\mathrm{H}$ & -1.5599 & 2.483008 & -0.80244 \\
$\mathrm{H}$ & -0.45711 & 2.49843 & -2.17074 \\
$\mathrm{C}$ & 0.867658 & -1.51378 & -0.0779 \\
$\mathrm{C}$ & -2.76223 & -2.29853 & 0.50344 \\
$\mathrm{H}$ & -3.50847 & -2.28753 & 1.301929 \\
$\mathrm{H}$ & -2.85204 & -3.26245 & -0.00318 \\
$\mathrm{C}$ & 1.855798 & -0.39947 & -0.16736 \\
$\mathrm{C}$ & 0.111848 & 3.353363 & 1.106516 \\
$\mathrm{H}$ & -0.03271 & 2.469129 & 1.728783 \\
$\mathrm{H}$ & -0.83205 & 3.901817 & 1.090716 \\
$\mathrm{H}$ & 0.84943 & 3.987578 & 1.601601 \\
$\mathrm{C}$ & 1.187473 & 0.729487 & -1.00822 \\
$\mathrm{H}$ & 1.466333 & 0.59541 & -2.05694 \\
$\mathrm{C}$ & -0.95155 & 0.149767 & 0.150874 \\
$\mathrm{H}$ & -0.74092 & 0.642236 & 1.115713
\end{tabular}




\begin{tabular}{|c|c|c|}
\hline 2.120288 & 0.287702 & 1.186108 \\
\hline 2.444773 & 2.501291 & -1.1936 \\
\hline 2.865367 & -0.25059 & 1.771698 \\
\hline 0.537897 & 3.008965 & -0.31821 \\
\hline 0.749506 & 3.95328 & -0.82465 \\
\hline-3.36002 & 0.95622 & 0.46028 \\
\hline-3.13812 & 1.886823 & 0.97022 \\
\hline 2.565283 & 1.701574 & 0.810259 \\
\hline 2.42474 & 2.405091 & 1.629629 \\
\hline 3.633169 & 1.698809 & 0.584996 \\
\hline-2.44959 & 0.104818 & 0.006685 \\
\hline-3.07637 & -1.16726 & -0.50036 \\
\hline-2.65237 & -1.44083 & -1.46926 \\
\hline-1.35111 & -2.14993 & 1.117606 \\
\hline-1.4314 & -1.66118 & 2.096315 \\
\hline-0.89436 & -3.12445 & 1.287268 \\
\hline 5.243341 & -1.47392 & 0.351077 \\
\hline 5.71946 & -0.52822 & 0.112305 \\
\hline 5.823022 & -2.18197 & 0.930023 \\
\hline 3.16102 & -0.82618 & -0.86513 \\
\hline 2.890665 & -1.31916 & -1.8023 \\
\hline 3.732768 & 0.069028 & -1.12299 \\
\hline 4.013623 & -1.75025 & -0.05458 \\
\hline 3.561441 & -2.70555 & 0.19535 \\
\hline-4.56493 & -0.79826 & -0.62539 \\
\hline-4.7878 & -0.5397 & -1.66153 \\
\hline-5.22427 & -1.62199 & -0.34839 \\
\hline-4.76207 & 0.446548 & 0.267352 \\
\hline-5.42397 & 1.183029 & -0.19363 \\
\hline-5.20868 & 0.19529 & 1.235327 \\
\hline-0.47648 & -1.28238 & 0.225721 \\
\hline
\end{tabular}




\begin{tabular}{|c|c|c|c|}
\hline C & 1.76833 & 2.072024 & -0.45427 \\
\hline $\mathrm{N}$ & -0.27717 & 0.822683 & -0.96121 \\
\hline & 1.085816 & -2.70083 & -0.55145 \\
\hline & -0.17785 & -2.39123 & -0.67062 \\
\hline & 1.201778 & 0.319287 & 1.774168 \\
\hline
\end{tabular}

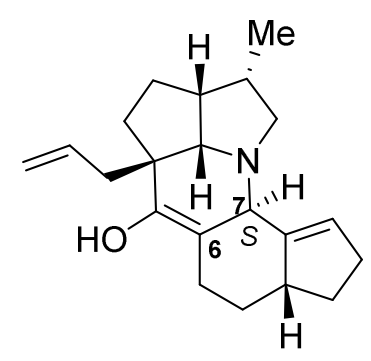

Level of theory: $\omega B 97 x-D /$ def2TZVP

Total Energy $=-947.67486873 \mathrm{au}$

RMS Gradient Norm $=0.00000318$ au

Imaginary Frequencies $=0$

Dipole Moment $=2.01$ Debye

$\begin{array}{lrrr}\text { Symbol } & \mathbf{X} & \mathbf{Y} & \mathbf{Z} \\ \mathrm{C} & 0.169107 & 2.354929 & -0.25748 \\ \text { H } & -0.04761 & 2.379138 & -1.34268 \\ \mathrm{H} & 1.094035 & 2.917631 & -0.10352 \\ \mathrm{C} & -0.51687 & -1.68778 & -0.24398 \\ \mathrm{C} & 2.993963 & -2.10322 & 0.325157 \\ \mathrm{H} & 3.946451 & -2.39755 & -0.1232 \\ \mathrm{H} & 2.820698 & -2.77404 & 1.169116 \\ \mathrm{C} & -1.59027 & -0.66012 & 0.037513 \\ \mathrm{C} & -1.58952 & 4.204236 & -0.0456 \\ \mathrm{H} & -1.88944 & 4.090403 & -1.08871 \\ \mathrm{H} & -0.85336 & 5.009237 & -0.00317 \\ \mathrm{H} & -2.46512 & 4.522924 & 0.523517 \\ \mathrm{C} & -0.96842 & 0.5266 & 0.794954 \\ \text { H } & -0.83251 & 0.261546 & 1.850164\end{array}$




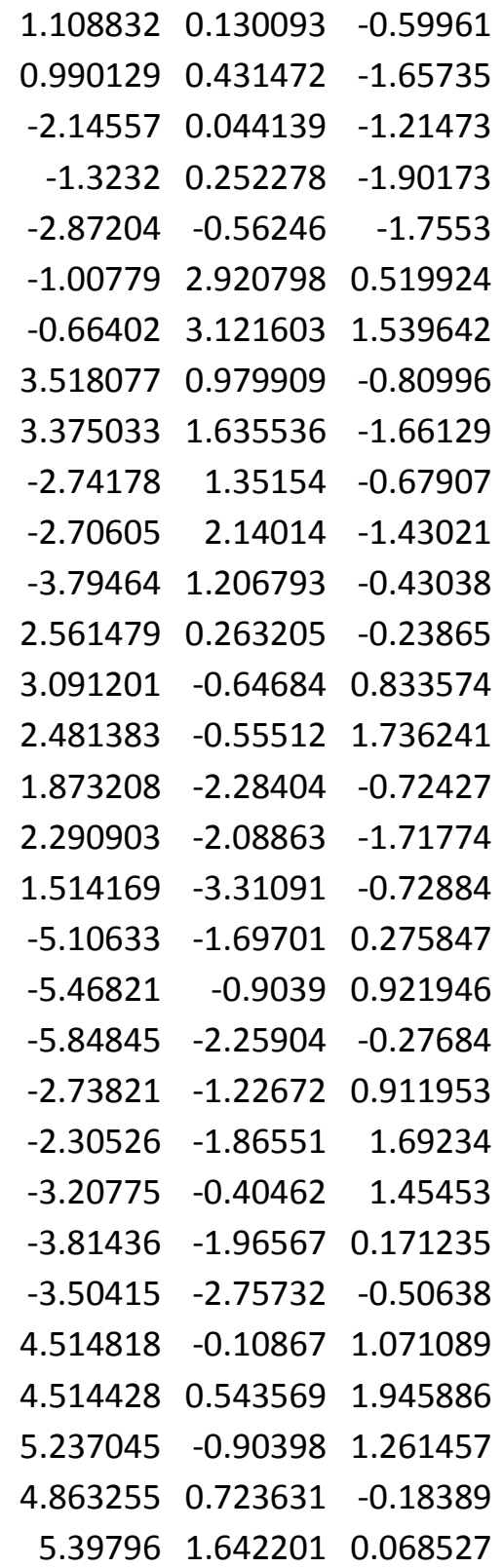




$\begin{array}{lrrr}\text { H } & 5.506605 & 0.171686 & -0.87741 \\ \mathrm{C} & 0.73769 & -1.32997 & -0.49308 \\ \mathrm{C} & -1.94227 & 1.706393 & 0.601794 \\ \mathrm{H} & -2.62077 & 1.815095 & 1.448863 \\ \mathrm{~N} & 0.293974 & 0.998238 & 0.23069 \\ \mathrm{O} & -0.85822 & -3.01624 & -0.2827 \\ \mathrm{H} & -1.57972 & -3.18858 & 0.322895\end{array}$

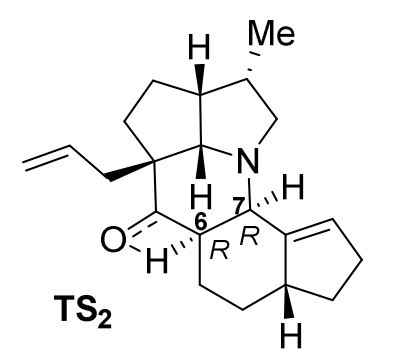

Level of theory: $\omega B$ 97x-D/def2TZVP

Total Energy $=-947.59041915 \mathrm{au}$

RMS Gradient Norm $=0.00000106$ au

Imaginary Frequencies $=1$
Dipole Moment $=2.47$ Debye

\begin{tabular}{lrrr} 
Symbol & $\mathbf{X}$ & \multicolumn{1}{c}{$\mathbf{Y}$} & $\mathbf{Z}$ \\
\hline $\mathrm{C}$ & -0.47095 & 2.589818 & 0.061676 \\
$\mathrm{H}$ & -0.86608 & 3.17605 & 0.895019 \\
$\mathrm{H}$ & -1.21342 & 2.626285 & -0.75284 \\
$\mathrm{C}$ & 0.736017 & -1.10596 & 1.137992 \\
$\mathrm{C}$ & -1.98763 & -2.24256 & -0.25116 \\
$\mathrm{H}$ & -2.72512 & -3.0434 & -0.3575 \\
$\mathrm{H}$ & -1.11393 & -2.55257 & -0.82896 \\
$\mathrm{C}$ & 1.511122 & -0.4522 & 0.041818 \\
$\mathrm{C}$ & 1.677475 & 3.739087 & 0.715123 \\
$\mathrm{H}$ & 1.815159 & 3.047056 & 1.547843 \\
$\mathrm{H}$ & 1.131736 & 4.607939 & 1.087631 \\
$\mathrm{H}$ & 2.658895 & 4.087869 & 0.391215
\end{tabular}




$\begin{array}{lrrr}\text { C } & 0.618573 & 0.643005 & -0.56218 \\ \text { H } & 0.007897 & 0.249296 & -1.39009 \\ \text { C } & -1.24737 & 0.421939 & 1.042015 \\ \text { H } & -1.58696 & 0.94217 & 1.94436 \\ \text { C } & 2.743067 & 0.33284 & 0.524155 \\ \text { H } & 2.507427 & 0.811007 & 1.475803 \\ \text { H } & 3.608893 & -0.30975 & 0.678479 \\ \text { C } & 0.898828 & 3.085375 & -0.42054 \\ \text { H } & 0.768107 & 3.827169 & -1.21064 \\ \text { C } & -3.53609 & 1.000546 & 0.105583 \\ \text { H } & -3.68205 & 1.896039 & 0.698487 \\ \text { C } & 2.952374 & 1.386566 & -0.5633 \\ \text { H } & 3.552127 & 2.225259 & -0.21438 \\ \text { H } & 3.495007 & 0.944685 & -1.40192 \\ \text { C } & -2.45182 & 0.235269 & 0.150867 \\ \text { C } & -2.5619 & -0.92799 & -0.81516 \\ \text { H } & -2.01605 & -0.66911 & -1.73096 \\ \text { C } & -1.57349 & -2.11375 & 1.218739 \\ \text { H } & -2.46319 & -1.97295 & 1.839095 \\ \text { H } & -1.10917 & -3.04287 & 1.552068 \\ \text { C } & 4.06022 & -2.66449 & -1.27507 \\ \text { H } & 4.321988 & -2.06711 & -2.14264 \\ \text { H } & 4.782185 & -3.40382 & -0.95213 \\ \text { C } & 1.868125 & -1.50159 & -1.04127 \\ \text { H } & 0.947949 & -2.02314 & -1.32314 \\ \text { H } & 2.200169 & -0.96269 & -1.93242 \\ \text { C } & 2.909392 & -2.4988 & -0.64211 \\ \text { H } & 0.172262 & -1.58066 & 2.51549 \\ \text { C } & -4.06852 & -0.94713 & -1.12474 \\ \text { H } & -4.29196 & -1.32455 & -2.12328 \\ \text { H } & -4.56302 & -1.60456 & -0.40372\end{array}$




$\begin{array}{lrrr}\mathrm{C} & -4.54324 & 0.49637 & -0.89199 \\ \mathrm{H} & -4.50248 & 1.084947 & -1.81583 \\ \mathrm{H} & -5.57128 & 0.551486 & -0.52887 \\ \mathrm{C} & -0.63815 & -0.91893 & 1.443207 \\ \mathrm{C} & 1.538431 & 1.795915 & -1.02689 \\ \mathrm{H} & 1.518394 & 1.880759 & -2.1131 \\ \mathrm{~N} & -0.18349 & 1.241473 & 0.481195 \\ \mathrm{O} & 1.276147 & -1.88826 & 2.004788 \\ \mathrm{H} & 2.68495 & -3.10508 & 0.229704\end{array}$

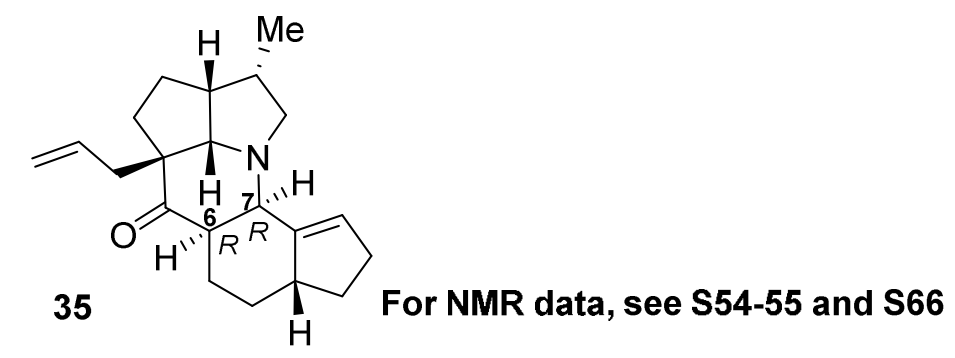
Level of theory: $\omega B$ 97x-D/def2TZVP Total Energy $=-947.70330770 \mathrm{au}$ RMS Gradient Norm $=0.00001106 \mathrm{au}$ Imaginary Frequencies $=0$ Dipole Moment $=3.02$ Debye

\begin{tabular}{lrcc} 
Symbol & $\mathbf{X}$ & $\mathbf{Y}$ & $\mathbf{Z}$ \\
\hline $\mathrm{C}$ & -0.77191 & 2.296183 & -0.10344 \\
$\mathrm{H}$ & -0.78699 & 2.563919 & 0.967498 \\
$\mathrm{H}$ & -1.7668 & 2.523355 & -0.49585 \\
$\mathrm{C}$ & 1.144206 & -1.12513 & 0.977861 \\
$\mathrm{C}$ & -2.10323 & -2.35369 & -0.77913 \\
$\mathrm{H}$ & -2.44868 & -2.99007 & 0.042854 \\
$\mathrm{H}$ & -2.30512 & -2.89465 & -1.70678 \\
$\mathrm{C}$ & 1.895686 & -0.18634 & 0.043429 \\
$\mathrm{C}$ & 0.498583 & 4.500798 & -0.34402 \\
$\mathrm{H}$ & 0.737155 & 4.533431 & 0.721106
\end{tabular}




$\begin{array}{lrrr}\text { H } & -0.41734 & 5.074891 & -0.49718 \\ \text { H } & 1.302307 & 5.004128 & -0.88494 \\ \mathrm{C} & 0.947286 & 0.746536 & -0.76321 \\ \mathrm{H} & 0.923674 & 0.41685 & -1.80871 \\ \mathrm{C} & -1.01107 & 0.006721 & 0.704267 \\ \mathrm{H} & -0.89596 & 0.433252 & 1.716953 \\ \mathrm{C} & 2.832497 & 0.767503 & 0.827594 \\ \mathrm{H} & 3.00749 & 0.414065 & 1.842125 \\ \mathrm{H} & 3.803678 & 0.80969 & 0.327525 \\ \mathrm{C} & 0.327523 & 3.07055 & -0.82546 \\ \mathrm{H} & 0.088497 & 3.085817 & -1.89351 \\ \mathrm{C} & -3.51215 & 0.326101 & 1.06576 \\ \mathrm{H} & -3.44476 & 0.986114 & 1.922774 \\ \mathrm{C} & 2.169738 & 2.138851 & 0.754994 \\ \mathrm{H} & 1.394618 & 2.222614 & 1.522728 \\ \mathrm{H} & 2.873159 & 2.956565 & 0.914003 \\ \mathrm{C} & -2.47111 & -0.18619 & 0.425825 \\ \mathrm{C} & -2.88422 & -1.03912 & -0.74722 \\ \mathrm{H} & -2.66 & -0.48964 & -1.66986 \\ \mathrm{C} & -0.60188 & -2.11747 & -0.62423 \\ \mathrm{H} & -0.06892 & -3.07088 & -0.61321 \\ \mathrm{H} & -0.23549 & -1.55215 & -1.48427 \\ \mathrm{C} & 3.793318 & -3.27382 & -0.5652 \\ \mathrm{H} & 3.111339 & -3.76673 & -1.25031 \\ \mathrm{H} & 4.544113 & -3.89207 & -0.09013 \\ \mathrm{C} & 2.702006 & -1.06622 & -0.94799 \\ \mathrm{H} & 2.021163 & -1.65468 & -1.56688 \\ \mathrm{H} & 3.217038 & -0.37257 & -1.62265 \\ \mathrm{C} & 3.705594 & -1.9774 & -0.31606 \\ \mathrm{H} & 4.398005 & -1.52643 & 0.388158 \\ \mathrm{C} & -4.40539 & -1.18524 & -0.55 \\ & & & \\ & & & \end{array}$




$\begin{array}{lrrr}\mathrm{H} & -4.95682 & -1.15418 & -1.48958 \\ \mathrm{H} & -4.61026 & -2.15324 & -0.0863 \\ \mathrm{C} & -4.81585 & -0.05921 & 0.42144 \\ \mathrm{H} & -5.24687 & 0.798371 & -0.10627 \\ \mathrm{H} & -5.56615 & -0.38695 & 1.143934 \\ \mathrm{C} & -0.31451 & -1.3617 & 0.680087 \\ \mathrm{H} & -0.7038 & -1.95023 & 1.513125 \\ \mathrm{C} & 1.535512 & 2.155383 & -0.63759 \\ \mathrm{H} & 2.306519 & 2.336376 & -1.39139 \\ \mathrm{~N} & -0.43492 & 0.89615 & -0.28586 \\ \mathrm{O} & 1.683224 & -1.63008 & 1.931217\end{array}$

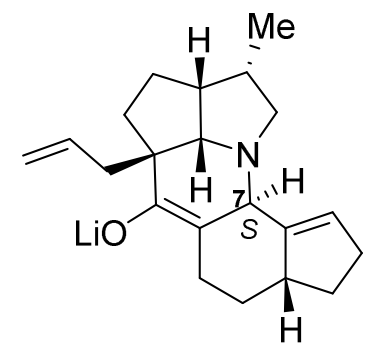

Level of theory: $\omega B$ 97x-D/def2TZVP

Total Energy $=-954.70235804 \mathrm{au}$

RMS Gradient Norm $=0.00000682 \mathrm{au}$

Imaginary Frequencies $=0$

Dipole Moment $=9.30$ Debye

\begin{tabular}{lrrl} 
Symbol & \multicolumn{1}{l}{$\mathbf{Y}$} & $\mathbf{Z}$ \\
\hline $\mathrm{C}$ & -1.23146 & -2.26073 & 0.811845 \\
H & -1.49806 & -1.64377 & 1.686319 \\
H & -0.8182 & -3.20097 & 1.185753 \\
$\mathrm{C}$ & -0.25952 & 1.082021 & 0.991455 \\
$\mathrm{C}$ & 3.254407 & 0.082552 & 1.601086 \\
H & 3.471554 & -0.9104 & 2.009532 \\
H & 4.075846 & 0.732693 & 1.912128 \\
$\mathrm{C}$ & -1.23915 & 0.77679 & -0.13669
\end{tabular}




\begin{tabular}{lrrr} 
C & -3.72958 & -2.73732 & 0.687835 \\
H & -3.96506 & -1.9198 & 1.372824 \\
H & -3.6301 & -3.64776 & 1.282955 \\
H & -4.58028 & -2.87052 & 0.016589 \\
C & -1.02699 & -0.61249 & -0.83036 \\
H & -0.50718 & -0.45408 & -1.77862 \\
C & 0.909848 & -1.09825 & 0.571888 \\
H & 1.176776 & -1.84517 & 1.335481 \\
C & -2.70154 & 0.736867 & 0.347924 \\
H & -2.74046 & 0.248209 & 1.325433 \\
C & -2.44741 & -2.4707 & -0.08278 \\
H & -3.14293 & 1.725511 & 0.471742 \\
H & -2.24033 & -3.34531 & -0.70466 \\
C & 2.448843 & -1.65805 & -1.43758 \\
H & 1.82572 & -2.37788 & -1.95332 \\
C & -3.42432 & -0.11247 & -0.69001 \\
H & -4.39116 & -0.48085 & -0.34702 \\
H & -3.61952 & 0.481368 & -1.58653 \\
C & 2.109861 & -1.00537 & -0.33248 \\
C & 3.188442 & -0.02861 & 0.082828 \\
H & 2.916858 & 0.960729 & -0.31317 \\
$\mathrm{C}$ & 1.910048 & 0.613945 & 2.160278 \\
H & 1.790538 & 0.252874 & 3.190444 \\
H & 1.9449 & 1.704507 & 2.223463 \\
$\mathrm{C}$ & -0.58146 & 4.294046 & -1.30139 \\
H & 0.355121 & 4.144857 & -1.82923 \\
H & -0.87363 & 5.31647 & -1.09265 \\
$\mathrm{C}$ & -1.04953 & 1.836115 & -1.25379 \\
$\mathrm{H}$ & -0.02685 & 1.754357 & -1.63412 \\
$\mathrm{H}$ & -1.70504 & 1.557121 & -2.08796 \\
$\mathrm{C}$ & -1.33933 & 3.266487 & -0.9312 \\
& & & \\
\hline
\end{tabular}




$\begin{array}{lrrr}\mathrm{H} & -2.27006 & 3.47177 & -0.41115 \\ \mathrm{C} & 4.414352 & -0.55595 & -0.66845 \\ \mathrm{H} & 5.139803 & 0.221851 & -0.91125 \\ \mathrm{H} & 4.91939 & -1.30222 & -0.04702 \\ \mathrm{C} & 3.823592 & -1.24927 & -1.91294 \\ \mathrm{H} & 3.758201 & -0.55411 & -2.75878 \\ \mathrm{H} & 4.427247 & -2.09486 & -2.24996 \\ \mathrm{C} & -2.43709 & -1.23312 & -1.02744 \\ \mathrm{C} & 0.750062 & 0.231104 & 1.27425 \\ \mathrm{H} & -2.58521 & -1.57032 & -2.05325 \\ \mathrm{~N} & -0.28449 & -1.60451 & -0.06871 \\ \mathrm{O} & -0.45368 & 2.223365 & 1.598775 \\ \mathrm{Li} & 0.120167 & 3.90159 & 1.296978\end{array}$

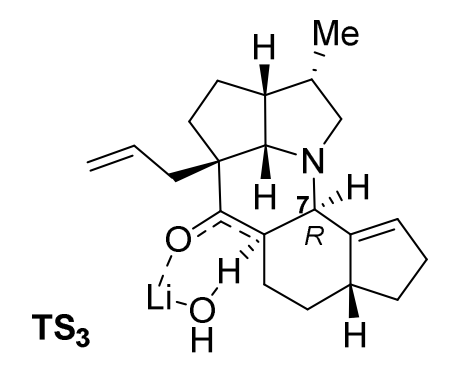

Level of theory: $\omega B$ 97x-D/def2TZVP

Total Energy $=-1031.14070843 \mathrm{au}$

RMS Gradient Norm $=0.00000546$ au

Imaginary Frequencies $=1$

Dipole Moment $=3.42$ Debye

\begin{tabular}{llll} 
Symbol & $\mathbf{X}$ & $\mathbf{Y}$ & $\mathbf{Z}$ \\
\hline $\mathrm{C}$ & -0.50849 & 2.514422 & 0.186339 \\
$\mathrm{H}$ & -0.41582 & 2.499265 & 1.287829 \\
$\mathrm{H}$ & -1.47955 & 2.961269 & -0.04359 \\
$\mathrm{C}$ & 0.813483 & -1.43826 & -0.15293 \\
$\mathrm{C}$ & -2.27367 & -1.49038 & -1.60809 \\
$\mathrm{H}$ & -2.93823 & -2.18152 & -2.1345
\end{tabular}




$\begin{array}{lrrr}\text { H } & -1.52316 & -1.15725 & -2.32994 \\ \text { C } & 1.718304 & -0.21623 & -0.18829 \\ \text { C } & 0.987321 & 4.588739 & 0.291543 \\ \text { H } & 1.208692 & 4.431476 & 1.348785 \\ \text { H } & 0.148087 & 5.284746 & 0.230984 \\ \text { H } & 1.853475 & 5.07001 & -0.16698 \\ \text { C } & 0.973676 & 0.950817 & -0.86046 \\ \text { H } & 0.95627 & 0.805782 & -1.94683 \\ \text { C } & -1.04579 & 0.161656 & 0.46939 \\ \text { H } & -0.88163 & 0.422831 & 1.529825 \\ \text { C } & 2.023529 & 0.393284 & 1.194272 \\ \text { H } & 1.129586 & 0.37179 & 1.817304 \\ \text { C } & 0.653657 & 3.28686 & -0.41521 \\ \text { H } & 0.402117 & 3.519502 & -1.45525 \\ \text { H } & -3.33159 & 0.953048 & 2.065134 \\ \text { C } & -3.49064 & 0.559031 & 1.06785 \\ \text { H } & 2.126233 & 2.509908 & 1.702587 \\ \text { C } & 2.422483 & 1.846925 & 0.889487 \\ \text { H } & 3.505938 & 1.932065 & 0.794862 \\ \text { H } & -3.0205 & 0.527526 & -1.81921 \\ \text { C } & -2.52375 & 0.204547 & 0.231184 \\ \text { C } & -3.074 & -0.28583 & -1.08709 \\ \text { H } & -2.30778 & -2.54384 & 0.2691 \\ \text { C } & -1.56134 & -2.19444 & -0.45303 \\ \text { H } & -1.03579 & -3.08107 & -0.80839 \\ \text { H } & 4.269072 & -2.95818 & -1.31114 \\ \mathrm{C} & 4.675069 & -2.32348 & -0.53103 \\ \text { H } & 5.528705 & -2.69956 & 0.020234 \\ \text { H } & 2.736976 & -1.1332 & -1.8545 \\ \text { C } & 3.003349 & -0.51116 & -0.99946 \\ \text { H } & 3.369434 & 0.437513 & -1.40285\end{array}$




$\begin{array}{lrrr}\text { H } & 4.589171 & -0.54433 & 0.532586 \\ \mathrm{C} & 4.142894 & -1.14131 & -0.25877 \\ \mathrm{H} & -5.21804 & -0.4236 & -1.58122 \\ \mathrm{C} & -4.54494 & -0.56349 & -0.73437 \\ \mathrm{H} & -4.63676 & -1.60344 & -0.4062 \\ \mathrm{H} & -5.26894 & 1.322922 & 0.116343 \\ \mathrm{C} & -4.85371 & 0.366166 & 0.453877 \\ \mathrm{H} & -5.57891 & -0.06303 & 1.148299 \\ \mathrm{H} & 2.505264 & 2.483313 & -1.19551 \\ \mathrm{C} & 1.749041 & 2.217341 & -0.45699 \\ \mathrm{C} & -0.55285 & -1.26462 & 0.226782 \\ \mathrm{H} & 2.79199 & -0.15726 & 1.735054 \\ \mathrm{~N} & -0.39196 & 1.171706 & -0.3626 \\ \mathrm{O} & 1.285644 & -2.56759 & -0.3962 \\ \mathrm{Li} & 0.85183 & -3.87862 & 1.095677 \\ \mathrm{H} & -0.36562 & -1.90551 & 1.382068 \\ \mathrm{O} & -0.08763 & -2.75577 & 2.32319 \\ \mathrm{H} & -0.91646 & -3.02396 & 2.720231\end{array}$

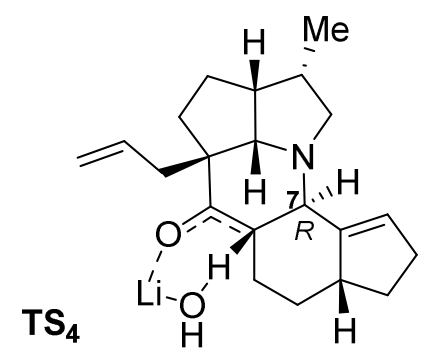

Level of theory: $\omega B 97 x-D /$ def2TZVP

Total Energy $=-1031.13969086 \mathrm{au}$

RMS Gradient Norm $=0.00000253 \mathrm{au}$

Imaginary Frequencies $=1$

Dipole Moment $=3.62$ Debye

\begin{tabular}{llrr} 
Symbol & \multicolumn{1}{l}{ X } & \multicolumn{1}{l}{ Y } \\
\hline C & -0.10412 & 2.612784 & 0.398374 \\
H & 0.166259 & 2.575308 & 1.470491 \\
H & -1.04217 & 3.169584 & 0.324
\end{tabular}




$\begin{array}{lrrr}\text { C } & 0.75297 & -1.32011 & 0.635103 \\ \text { C } & -3.0215 & -1.51868 & 1.389384 \\ \text { H } & -3.19081 & -0.79515 & 2.193759 \\ \text { H } & -3.67264 & -2.37171 & 1.593067 \\ \text { C } & 1.730723 & -0.28923 & 0.10313 \\ \text { C } & 1.583574 & 4.529299 & 0.171684 \\ \text { H } & 1.902126 & 4.405651 & 1.208076 \\ \text { H } & 0.830457 & 5.319614 & 0.153395 \\ \text { H } & 2.443169 & 4.874117 & -0.40668 \\ \text { C } & 1.053378 & 0.835141 & -0.70779 \\ \text { H } & 0.937428 & 0.508338 & -1.74722 \\ \text { C } & -1.13428 & 0.37579 & 0.453669 \\ \text { H } & -1.26407 & 0.719936 & 1.496849 \\ \text { C } & 2.364559 & 0.489858 & 1.268515 \\ \text { H } & 2.585228 & 2.207735 & -1.48434 \\ \text { C } & 1.016872 & 3.245276 & -0.4084 \\ \text { H } & 3.122235 & -0.08208 & 1.803787 \\ \text { H } & 0.613524 & 3.470393 & -1.40059 \\ \text { C } & -3.18853 & 1.312164 & -0.7967 \\ \text { H } & -2.78406 & 2.269091 & -1.1024 \\ \text { C } & 2.912269 & 1.762631 & 0.620479 \\ \text { H } & 2.960718 & 2.584286 & 1.33381 \\ \text { H } & 3.93209 & 1.595432 & 0.271034 \\ \text { C } & 1.988596 & 2.066541 & -0.58331 \\ \text { C } & -3.3716 & -0.85931 & 0.065624 \\ \text { H } & -3.13548 & -1.57335 & -0.737 \\ \text { C } & -1.55146 & -1.97339 & 1.374456 \\ \text { H } & -1.17594 & -2.04497 & 2.400203 \\ \text { H } & -1.50293 & -2.98464 & 0.963975 \\ \text { C } & 4.113139 & -3.05212 & -0.8451 \\ \text { H } & 3.691882 & -3.35279 & -1.79937\end{array}$




$\begin{array}{lrrr}\text { H } & 4.849268 & -3.71137 & -0.40155 \\ \mathrm{C} & 2.77574 & -0.94227 & -0.82865 \\ \mathrm{H} & 2.243595 & -1.40418 & -1.66536 \\ \mathrm{H} & 3.368812 & -0.1302 & -1.2636 \\ \mathrm{C} & 3.733985 & -1.93241 & -0.24462 \\ \mathrm{H} & 4.182637 & -1.67494 & 0.710512 \\ \mathrm{C} & -4.79002 & -0.32093 & -0.14066 \\ \mathrm{H} & -5.48537 & -1.07183 & -0.51803 \\ \mathrm{H} & -5.17527 & 0.041866 & 0.817364 \\ \mathrm{C} & -4.60208 & 0.873229 & -1.09755 \\ \mathrm{H} & -4.70365 & 0.56203 & -2.14417 \\ \mathrm{H} & -5.3373 & 1.663879 & -0.93363 \\ \mathrm{C} & -0.64113 & -1.06399 & 0.539605 \\ \mathrm{C} & -2.52099 & 0.371437 & -0.14017 \\ \mathrm{H} & -0.63437 & -1.6953 & -0.63836 \\ \mathrm{~N} & -0.22801 & 1.298223 & -0.19112 \\ \mathrm{O} & 1.192056 & -2.43087 & 1.002793 \\ \mathrm{Li} & 0.743404 & -3.68649 & -0.61123 \\ \mathrm{O} & -0.46992 & -2.58558 & -1.60266 \\ \mathrm{H} & 1.584063 & 0.740488 & 1.992534 \\ \mathrm{H} & -1.32127 & -2.92211 & -1.88261\end{array}$


Level of theory: $\omega B$ 97x-D/def2TZVP

Total Energy $=-1031.14406428$ au

\begin{tabular}{lrrl} 
Symbol & \multicolumn{1}{l}{ X } & \multicolumn{2}{l}{$Z$} \\
\hline $\mathrm{C}$ & 1.63825 & -2.07039 & -1.14647 \\
$\mathrm{H}$ & 1.773242 & -1.29303 & -1.93166 \\
$\mathrm{H}$ & 1.35906 & -3.00516 & -1.65949 \\
$\mathrm{C}$ & 0.111187 & 1.025871 & -0.70162 \\
$\mathrm{C}$ & -3.23607 & -0.42673 & -1.37884 \\
$\mathrm{H}$ & -3.33029 & -1.37087 & -1.94291 \\
$\mathrm{H}$ & -4.175 & 0.125397 & -1.54007 \\
$\mathrm{C}$ & 1.191671 & 0.733693 & 0.320011 \\
$\mathrm{C}$ & 4.19289 & -2.16725 & -1.1264 \\
$\mathrm{H}$ & 4.283663 & -1.22052 & -1.6817 \\
$\mathrm{H}$ & 4.214366 & -2.98318 & -1.86494 \\
$\mathrm{H}$ & 5.084332 & -2.27231 & -0.4891 \\
$\mathrm{C}$ & 1.240841 & -0.77419 & 0.764166 \\
$\mathrm{H}$ & 0.735798 & -0.87578 & 1.738604 \\
$\mathrm{C}$ & -0.65659 & -1.33467 & -0.65483 \\
$\mathrm{H}$ & -0.8503 & -1.96758 & -1.54276 \\
$\mathrm{C}$ & 2.612298 & 1.020551 & -0.21382 \\
$\mathrm{H}$ & 2.667266 & 0.703753 & -1.26742 \\
$\mathrm{C}$ & 2.909444 & -2.22309 & -0.31111 \\
$\mathrm{H}$ & 2.881633 & 2.086356 & -0.20034 \\
$\mathrm{H}$ & 2.85168 & -3.21073 & 0.172936
\end{tabular}




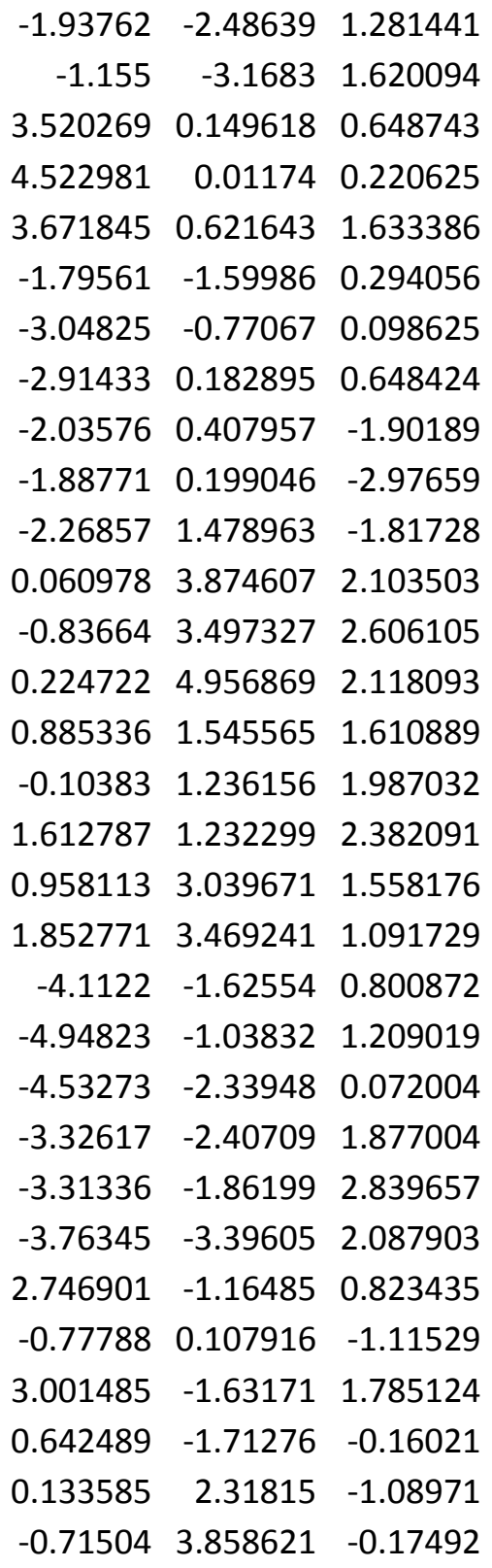




\begin{tabular}{|c|c|c|}
\hline-1.70582 & 3.710161 & -1.58203 \\
\hline-2.24791 & 3.946266 & -2.33085 \\
\hline-1.03693 & 2.945577 & -1.73148 \\
\hline
\end{tabular}

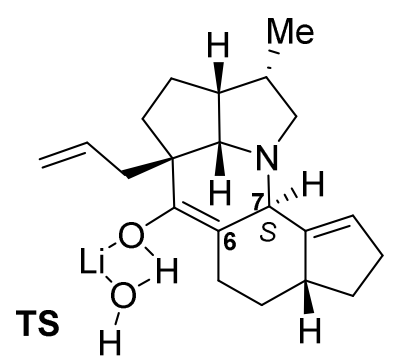

Level of theory: $\omega B 97 x-D / d e f 2 T Z V P$

Total Energy $=-1031.14039603 \mathrm{au}$

\begin{tabular}{lrrr} 
Symbol & $\mathbf{X}$ & $\mathbf{Y}$ & $\mathbf{Z}$ \\
\hline $\mathrm{C}$ & 1.631517 & -2.07599 & -1.14373 \\
$\mathrm{H}$ & 1.769378 & -1.29993 & -1.92971 \\
$\mathrm{H}$ & 1.34983 & -3.01048 & -1.65588 \\
$\mathrm{C}$ & 0.113558 & 1.025386 & -0.70336 \\
$\mathrm{C}$ & -3.23764 & -0.41782 & -1.38114 \\
$\mathrm{H}$ & -3.33436 & -1.3623 & -1.94421 \\
$\mathrm{H}$ & -4.17479 & 0.136966 & -1.54361 \\
$\mathrm{C}$ & 1.192484 & 0.731082 & 0.319311 \\
$\mathrm{C}$ & 4.185838 & -2.18057 & -1.12187 \\
$\mathrm{H}$ & 4.279844 & -1.23474 & -1.67818 \\
$\mathrm{H}$ & 4.205322 & -2.99739 & -1.85948 \\
$\mathrm{H}$ & 5.076541 & -2.28761 & -0.48387 \\
$\mathrm{C}$ & 1.236793 & -0.77644 & 0.765189 \\
$\mathrm{H}$ & 0.730807 & -0.8754 & 1.73941 \\
$\mathrm{C}$ & -0.6614 & -1.33276 & -0.65442 \\
$\mathrm{H}$ & -0.85645 & -1.96608 & -1.54177
\end{tabular}




$\begin{array}{lrrr}\text { C } & 2.614322 & 1.013033 & -0.21391 \\ \text { H } & 2.669018 & 0.694885 & -1.26711 \\ \text { C } & 2.901697 & -2.2316 & -0.30736 \\ \text { H } & 2.886879 & 2.078031 & -0.20145 \\ \text { H } & 2.840622 & -3.21852 & 0.177751 \\ \text { C } & -1.94718 & -2.47842 & 1.282299 \\ \text { H } & -1.16685 & -3.16231 & 1.622229 \\ \text { C } & 3.519086 & 0.140322 & 0.650226 \\ \text { H } & 4.521654 & -0.00108 & 0.222922 \\ \text { H } & 3.671449 & 0.612992 & 1.634439 \\ \text { C } & -1.80184 & -1.59343 & 0.294013 \\ \text { C } & -3.05183 & -0.76067 & 0.096829 \\ \text { H } & -2.91538 & 0.193102 & 0.645647 \\ \text { C } & -2.03446 & 0.412634 & -1.90434 \\ \text { H } & -1.88635 & 0.202067 & -2.97871 \\ \text { H } & -2.26408 & 1.484435 & -1.82108 \\ \text { C } & 0.070155 & 3.877411 & 2.098535 \\ \text { H } & -0.82894 & 3.503419 & 2.600971 \\ \text { H } & 0.237169 & 4.959188 & 2.112019 \\ \text { C } & 0.887769 & 1.545329 & 1.609076 \\ \text { H } & -0.10258 & 1.239343 & 1.984917 \\ \text { H } & 1.613763 & 1.230726 & 2.381107 \\ \text { C } & 0.965109 & 3.039148 & 1.554735 \\ \text { H } & 1.86137 & 3.465479 & 1.088394 \\ \text { C } & -4.11883 & -1.61152 & 0.799336 \\ \text { H } & -4.95334 & -1.02132 & 1.206275 \\ \text { H } & -4.54105 & -2.325 & 0.070993 \\ \text { C } & -3.33588 & -2.39424 & 1.87686 \\ \text { H } & -3.32204 & -1.8481 & 2.838909 \\ \text { H } & -3.77629 & -3.38163 & 2.088581 \\ \mathrm{C} & 2.741622 & -1.1716 & 0.825885\end{array}$




\begin{tabular}{|c|c|c|c|}
\hline C & -0.77802 & 0.109665 & -1.11658 \\
\hline $\mathrm{H}$ & 2.994161 & -1.63814 & 1.788265 \\
\hline$N$ & 0.636201 & -1.71423 & -0.15853 \\
\hline U & 0.140128 & 2.317154 & -1.09288 \\
\hline & -0.70442 & 3.861217 & -0.18038 \\
\hline Q & -1.69473 & 3.71418 & -1.58797 \\
\hline $\mathrm{H}$ & -2.23561 & 3.951085 & -2.33741 \\
\hline & -0.81026 & 2.8299 & -1.61619 \\
\hline
\end{tabular}

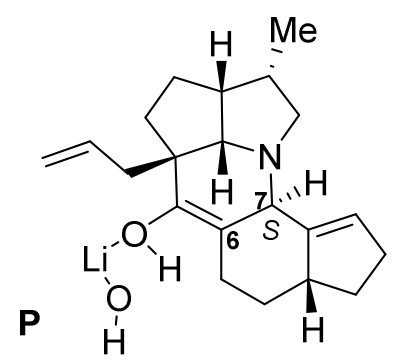

Level of theory: $\omega B$ 97x-D/def2TZVP

Total Energy $=-1031.14171934 \mathrm{au}$

\begin{tabular}{lrrr} 
Symbol & \multicolumn{1}{l}{ X } & \multicolumn{2}{l}{$Z$} \\
\hline $\mathrm{C}$ & 1.627481 & -2.07876 & -1.1424 \\
$\mathrm{H}$ & 1.766943 & -1.30329 & -1.92868 \\
$\mathrm{H}$ & 1.344287 & -3.01298 & -1.65422 \\
$\mathrm{C}$ & 0.11489 & 1.025494 & -0.70384 \\
$\mathrm{C}$ & -3.23866 & -0.41208 & -1.38196 \\
$\mathrm{H}$ & -3.33689 & -1.35664 & -1.94464 \\
$\mathrm{H}$ & -4.17479 & 0.144297 & -1.54494 \\
$\mathrm{C}$ & 1.192997 & 0.729734 & 0.319269 \\
$\mathrm{C}$ & 4.181606 & -2.18785 & -1.11976 \\
$\mathrm{H}$ & 4.277448 & -1.24244 & -1.67645 \\
$\mathrm{H}$ & 4.199857 & -3.00503 & -1.85699 \\
$\mathrm{H}$ & 5.071934 & -2.29619 & -0.48145
\end{tabular}




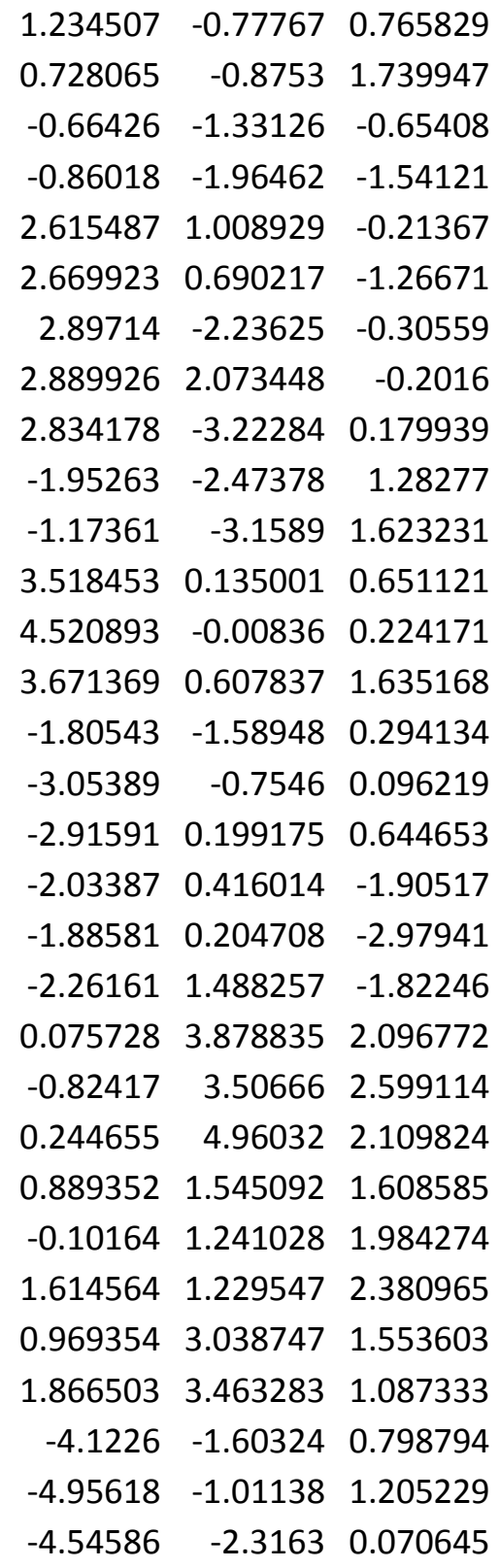




$\begin{array}{lrrr}\mathrm{C} & -3.34134 & -2.38688 & 1.876892 \\ \mathrm{H} & -3.32682 & -1.84033 & 2.838703 \\ \mathrm{H} & -3.78357 & -3.37339 & 2.088923 \\ \mathrm{C} & 2.738616 & -1.17546 & 0.827137 \\ \mathrm{C} & -0.77819 & 0.11117 & -1.11692 \\ \mathrm{H} & 2.99005 & -1.64203 & 1.789796 \\ \mathrm{~N} & 0.632522 & -1.7148 & -0.15765 \\ \mathrm{O} & 0.14386 & 2.317039 & -1.09393 \\ \mathrm{Li} & -0.69821 & 3.863001 & -0.18236 \\ \mathrm{O} & -1.68837 & 3.717093 & -1.59017 \\ \mathrm{H} & -2.22862 & 3.954623 & -2.33988 \\ \mathrm{H} & -0.68035 & 2.717863 & -1.6063\end{array}$

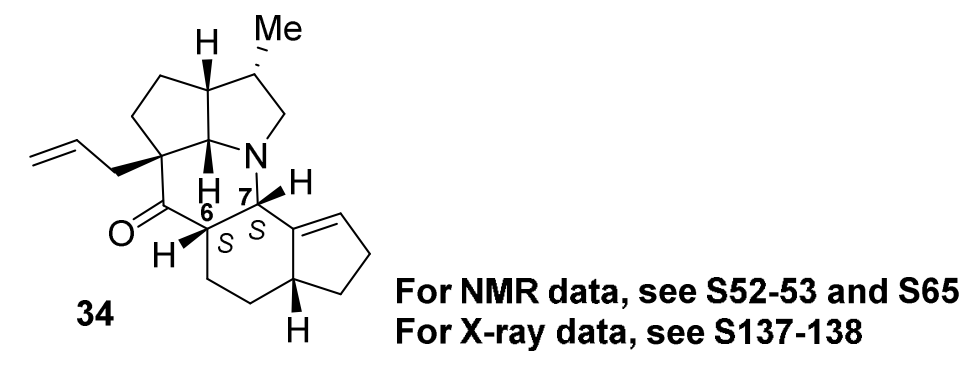
Level of theory: $\omega B 97 x-D / d e f 2 T Z V P$ Total Energy $=-947.70653980 \mathrm{au}$ RMS Gradient Norm $=0.00000615$ au Imaginary Frequencies $=0$
Dipole Moment $=2.86$ Debye

\begin{tabular}{llll} 
Symbol & $\mathbf{X}$ & $\mathbf{Y}$ & $\mathbf{Z}$ \\
\hline $\mathrm{C}$ & -0.15872 & 2.441195 & -1.18503 \\
H & -1.07522 & 2.917902 & -0.83421 \\
$\mathrm{H}$ & -0.13705 & 2.525627 & -2.28688 \\
$\mathrm{C}$ & 0.355501 & -1.40883 & 0.111456 \\
$\mathrm{C}$ & -2.86904 & -2.10157 & -0.11391 \\
H & -2.50863 & -2.16351 & 0.915881 \\
H & -3.63576 & -2.8702 & -0.24075 \\
C & 1.587554 & -0.52453 & -0.03616
\end{tabular}




$\begin{array}{lrrr}\mathrm{C} & 0.819856 & 3.656176 & 0.802502 \\ \mathrm{H} & 0.436618 & 2.905491 & 1.496761 \\ \mathrm{H} & 0.070964 & 4.445164 & 0.711792 \\ \mathrm{H} & 1.712334 & 4.100911 & 1.244654 \\ \mathrm{C} & 1.266569 & 0.630672 & -0.99152 \\ \mathrm{H} & 1.44643 & 0.363714 & -2.04805 \\ \mathrm{C} & -1.14691 & 0.156653 & -1.12279 \\ \mathrm{H} & -1.48347 & 0.326916 & -2.16247 \\ \mathrm{C} & 1.967905 & 0.21058 & 1.256518 \\ \mathrm{H} & 1.054294 & 0.529148 & 1.760506 \\ \mathrm{H} & 2.526703 & -0.42052 & 1.945437 \\ \mathrm{C} & 1.108228 & 3.047932 & -0.56424 \\ \mathrm{H} & 1.491455 & 3.83874 & -1.21149 \\ \mathrm{C} & -2.46182 & 1.020905 & 0.877812 \\ \mathrm{H} & -1.71636 & 1.719238 & 1.234264 \\ \mathrm{C} & 2.763363 & 1.423751 & 0.770033 \\ \mathrm{H} & 2.792918 & 2.219651 & 1.511711 \\ \mathrm{H} & 3.800367 & 1.132918 & 0.588832 \\ \mathrm{C} & -2.33099 & 0.278372 & -0.21261 \\ \mathrm{C} & -3.45646 & -0.71068 & -0.36048 \\ \mathrm{H} & -3.8769 & -0.6919 & -1.37271 \\ \mathrm{C} & -1.71567 & -2.36403 & -1.07835 \\ \mathrm{H} & -1.26588 & -3.33621 & -0.87029 \\ \mathrm{H} & -2.11608 & -2.41078 & -2.09664 \\ \mathrm{C} & 4.573805 & -2.43045 & 0.730667 \\ \mathrm{H} & 5.303728 & -1.71763 & 0.360717 \\ \mathrm{H} & 4.928765 & -3.18148 & 1.425092 \\ \mathrm{C} & 2.751292 & -1.37354 & -0.60258 \\ \mathrm{H} & 2.395976 & -1.8805 & -1.50751 \\ \mathrm{H} & 3.54857 & -0.69815 & -0.92479 \\ \mathrm{C} & 3.306309 & -2.3831 & 0.351499\end{array}$




$\begin{array}{lrrr}\text { H } & 2.599991 & -3.10275 & 0.753542 \\ \mathrm{C} & -4.48875 & -0.23232 & 0.67764 \\ \mathrm{H} & -5.28249 & 0.322816 & 0.175535 \\ \mathrm{H} & -4.95969 & -1.06742 & 1.198186 \\ \mathrm{C} & -3.72177 & 0.70547 & 1.633417 \\ \mathrm{H} & -4.29798 & 1.597314 & 1.890589 \\ \mathrm{H} & -3.47666 & 0.212518 & 2.579776 \\ \mathrm{C} & -0.6132 & -1.30515 & -1.0565 \\ \mathrm{H} & 0.009405 & -1.4504 & -1.9466 \\ \mathrm{C} & 2.11348 & 1.849998 & -0.56558 \\ \mathrm{H} & 2.890167 & 2.049128 & -1.30336 \\ \mathrm{~N} & -0.08914 & 1.067562 & -0.75597 \\ \mathrm{O} & 0.193028 & -2.15171 & 1.046678\end{array}$

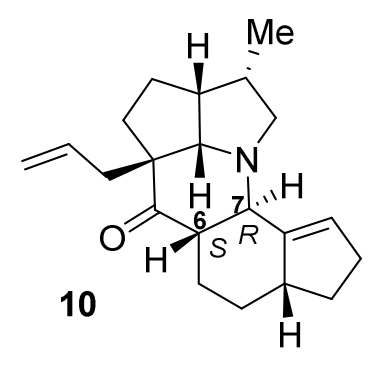

Level of theory: $\omega B$ 97x-D/def2TZVP

Total Energy $=-947.69583343 \mathrm{au}$

RMS Gradient Norm $=0.00000573$ au

Imaginary Frequencies $=0$

Dipole Moment $=2.23$ Debye

\begin{tabular}{lrrl} 
Symbol & \multicolumn{1}{l}{$\mathbf{X}$} & $\mathbf{Y}$ & $\mathbf{Z}$ \\
\hline $\mathrm{C}$ & -0.09347 & 2.421945 & 0.455906 \\
$\mathrm{H}$ & 0.096173 & 2.343284 & 1.541457 \\
$\mathrm{H}$ & -1.01917 & 2.992174 & 0.338414 \\
$\mathrm{C}$ & 0.822246 & -1.23519 & 0.804507 \\
$\mathrm{C}$ & -3.0091 & -1.81374 & 1.116906 \\
$\mathrm{H}$ & -3.2474 & -1.17234 & 1.971018 \\
$\mathrm{H}$ & -3.63042 & -2.70593 & 1.215349
\end{tabular}




$\begin{array}{lrrr}\text { C } & 1.770899 & -0.55655 & -0.17443 \\ \mathrm{C} & 1.686184 & 4.265259 & 0.487138 \\ \mathrm{H} & 2.03699 & 3.979005 & 1.480538 \\ \mathrm{H} & 0.950067 & 5.062024 & 0.611155 \\ \mathrm{H} & 2.53437 & 4.678505 & -0.06203 \\ \mathrm{C} & 1.07682 & 0.714046 & -0.77796 \\ \mathrm{H} & 0.943279 & 0.566467 & -1.85617 \\ \mathrm{C} & -1.13106 & 0.185684 & 0.372217 \\ \mathrm{H} & -1.26853 & 0.42388 & 1.441323 \\ \mathrm{C} & 3.052139 & -0.045 & 0.503956 \\ \mathrm{H} & 3.285097 & -0.61472 & 1.401937 \\ \mathrm{H} & 3.892068 & -0.16234 & -0.18551 \\ \mathrm{C} & 1.08339 & 3.082723 & -0.25056 \\ \mathrm{H} & 0.73328 & 3.419109 & -1.23127 \\ \mathrm{C} & -3.1637 & 1.180704 & -0.85447 \\ \mathrm{H} & -2.76189 & 2.158872 & -1.08401 \\ \mathrm{C} & 2.798656 & 1.431556 & 0.765314 \\ \mathrm{H} & 2.188611 & 1.548333 & 1.665774 \\ \mathrm{H} & 3.716344 & 1.999351 & 0.921038 \\ \mathrm{C} & -2.5016 & 0.205116 & -0.24916 \\ \mathrm{C} & -3.33389 & -1.05365 & -0.16008 \\ \mathrm{H} & -3.07074 & -1.69791 & -1.01262 \\ \mathrm{C} & -1.51799 & -2.2075 & 1.147961 \\ \mathrm{H} & -1.15385 & -2.23555 & 2.175267 \\ \mathrm{H} & -1.39437 & -3.21775 & 0.752187 \\ \mathrm{C} & 4.077858 & -3.10125 & -1.25174 \\ \mathrm{H} & 4.605611 & -2.50732 & -1.99074 \\ \mathrm{H} & 4.583958 & -3.98092 & -0.8747 \\ \mathrm{C} & 2.107235 & -1.56661 & -1.30026 \\ \mathrm{H} & 1.183109 & -1.89061 & -1.78902 \\ \mathrm{H} & 2.688671 & -1.03355 & -2.05745\end{array}$




$\begin{array}{llll}\mathrm{C} & 2.868959 & -2.76547 & -0.83037 \\ \mathrm{H} & 2.381788 & -3.38255 & -0.08024 \\ \mathrm{C} & -4.75444 & -0.51883 & -0.36053 \\ \mathrm{H} & -5.43072 & -1.24762 & -0.80889 \\ \mathrm{H} & -5.16708 & -0.23448 & 0.611979 \\ \mathrm{C} & -4.56194 & 0.746386 & -1.22035 \\ \mathrm{H} & -4.63361 & 0.516266 & -2.28984 \\ \mathrm{H} & -5.31208 & 1.512406 & -1.01451 \\ \mathrm{C} & -0.6173 & -1.26733 & 0.337624 \\ \mathrm{H} & -0.6113 & -1.57083 & -0.7137 \\ \mathrm{C} & 2.019398 & 1.892163 & -0.4703 \\ \mathrm{H} & 2.714745 & 2.068222 & -1.2944 \\ \mathrm{~N} & -0.19025 & 1.126969 & -0.17882 \\ \mathrm{O} & 1.17788 & -1.68364 & 1.865197\end{array}$

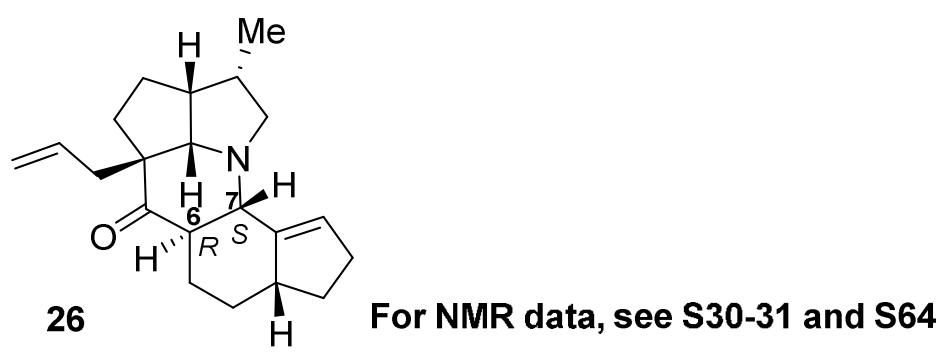
Level of theory: $\omega B$ 97x-D/def2TZVP
Total Energy $=-947.70504240 \mathrm{au}$
RMS Gradient Norm $=0.00000578 \mathrm{au}$
Imaginary Frequencies $=0$
Dipole Moment $=2.86$ Debye

\begin{tabular}{lrll} 
Symbol & \multicolumn{1}{l}{$\mathbf{X}$} & $\mathbf{Y}$ & $\mathbf{Z}$ \\
\hline $\mathrm{C}$ & -0.39618 & 2.237348 & -1.03387 \\
$\mathrm{H}$ & -1.31842 & 2.694269 & -0.68327 \\
$\mathrm{H}$ & -0.4512 & 2.177441 & -2.13517 \\
$\mathrm{C}$ & 0.796634 & -1.70127 & 0.000362 \\
$\mathrm{C}$ & -3.04996 & -2.19944 & 0.311825 \\
$\mathrm{H}$ & -3.15783 & -1.98348 & 1.380696
\end{tabular}




\begin{tabular}{lrrr} 
H & -3.69307 & -3.05542 & 0.093129 \\
$\mathrm{C}$ & 1.784958 & -0.54222 & -0.06946 \\
$\mathrm{C}$ & 0.596931 & 3.763471 & 0.709792 \\
$\mathrm{H}$ & 0.234678 & 3.08796 & 1.488042 \\
$\mathrm{H}$ & -0.16324 & 4.53038 & 0.548134 \\
$\mathrm{H}$ & 1.494431 & 4.261516 & 1.080219 \\
$\mathrm{C}$ & 1.17357 & 0.606979 & -0.87348 \\
$\mathrm{H}$ & 1.200836 & 0.393919 & -1.95804 \\
$\mathrm{C}$ & -1.11042 & -0.13581 & -0.64562 \\
$\mathrm{H}$ & -1.11827 & -0.45828 & -1.70698 \\
$\mathrm{C}$ & 2.040789 & 0.165683 & 1.277991 \\
$\mathrm{H}$ & 1.100962 & 0.286435 & 1.81566 \\
$\mathrm{H}$ & 2.731693 & -0.3904 & 1.910407 \\
$\mathrm{C}$ & 0.859242 & 3.004763 & -0.58408 \\
$\mathrm{H}$ & 1.147362 & 3.725313 & -1.35111 \\
$\mathrm{C}$ & -3.03362 & 1.096 & 0.526611 \\
$\mathrm{H}$ & -2.48107 & 1.93397 & 0.930248 \\
$\mathrm{C}$ & 2.591347 & 1.540653 & 0.865449 \\
$\mathrm{H}$ & 2.406539 & 2.289983 & 1.631922 \\
$\mathrm{H}$ & 3.674415 & 1.476219 & 0.748822 \\
$\mathrm{C}$ & -2.52231 & 0.140642 & -0.23911 \\
$\mathrm{C}$ & -3.51353 & -0.97117 & -0.48171 \\
$\mathrm{H}$ & -3.54847 & -1.24382 & -1.54188 \\
$\mathrm{C}$ & -1.59424 & -2.54617 & 0.006643 \\
$\mathrm{H}$ & -1.25848 & -3.37534 & 0.628809 \\
$\mathrm{H}$ & -1.50232 & -2.8898 & -1.02838 \\
$\mathrm{C}$ & 5.250216 & -1.42989 & 0.445296 \\
$\mathrm{H}$ & 5.681756 & -0.50527 & 0.07482 \\
$\mathrm{H}$ & 5.879337 & -2.04716 & 1.074417 \\
$\mathrm{C}$ & 3.099878 & -0.9925 & -0.74127 \\
$\mathrm{H}$ & 2.838739 & -1.58653 & -1.62136 \\
& & & \\
\hline
\end{tabular}




$\begin{array}{lrrr}\text { H } & 3.634389 & -0.1105 & -1.10175 \\ \mathrm{C} & 4.015135 & -1.79154 & 0.13258 \\ \mathrm{H} & 3.611891 & -2.72247 & 0.516053 \\ \mathrm{C} & -4.84874 & -0.3647 & -0.0158 \\ \mathrm{H} & -5.41299 & -0.01354 & -0.88076 \\ \mathrm{H} & -5.4722 & -1.09454 & 0.502533 \\ \mathrm{C} & -4.47258 & 0.834947 & 0.877874 \\ \mathrm{H} & -5.11557 & 1.700111 & 0.701281 \\ \mathrm{H} & -4.56359 & 0.597278 & 1.943047 \\ \mathrm{C} & -0.66787 & -1.35147 & 0.212941 \\ \mathrm{H} & -0.74788 & -1.00845 & 1.251869 \\ \mathrm{C} & 1.944625 & 1.884123 & -0.50107 \\ \mathrm{H} & 2.726451 & 2.094138 & -1.22995 \\ \mathrm{~N} & -0.17242 & 0.93327 & -0.44433 \\ \mathrm{O} & 1.148087 & -2.84843 & -0.13284\end{array}$

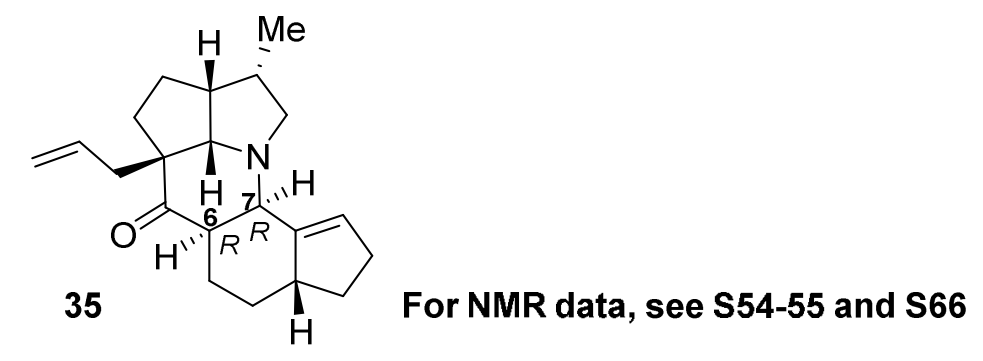
Level of theory: $\omega B$ 97x-D/def2TZVP/IEFPCM(THF)
Total Energy $=-947.70973506 \mathrm{au}$
RMS Gradient Norm $=0.00000501 \mathrm{au}$
Imaginary Frequencies $=0$
Dipole Moment $=3.97$ Debye

\begin{tabular}{lrrl} 
Symbol & \multicolumn{1}{l}{ X } & \multicolumn{1}{l}{$\mathbf{Z}$} \\
\hline $\mathrm{C}$ & -0.75286 & 2.306896 & -0.08273 \\
$\mathrm{H}$ & -0.7472 & 2.568542 & 0.989312 \\
$\mathrm{H}$ & -1.75038 & 2.549238 & -0.45881 \\
$\mathrm{C}$ & 1.134258 & -1.1317 & 0.976161 \\
$\mathrm{C}$ & -2.11706 & -2.34972 & -0.77304
\end{tabular}




$\begin{array}{lrrr}\text { H } & -2.46275 & -2.98022 & 0.05331 \\ \text { H } & -2.32092 & -2.89477 & -1.69757 \\ \text { C } & 1.890408 & -0.19753 & 0.046361 \\ \text { C } & 0.535809 & 4.49923 & -0.3396 \\ \text { H } & 0.790966 & 4.528627 & 0.721881 \\ \text { H } & -0.37634 & 5.082682 & -0.47989 \\ \text { H } & 1.336788 & 4.992789 & -0.89334 \\ \mathrm{C} & 0.945301 & 0.740789 & -0.75928 \\ \text { H } & 0.917536 & 0.406411 & -1.80247 \\ \mathrm{C} & -1.01741 & 0.013544 & 0.706225 \\ \mathrm{H} & -0.9042 & 0.437106 & 1.719844 \\ \mathrm{C} & 2.841622 & 0.751717 & 0.819654 \\ \mathrm{H} & 3.022094 & 0.406447 & 1.836159 \\ \mathrm{H} & 3.808701 & 0.781293 & 0.31202 \\ \mathrm{C} & 0.344423 & 3.070587 & -0.81823 \\ \mathrm{H} & 0.093253 & 3.087208 & -1.88323 \\ \mathrm{C} & -3.51813 & 0.344718 & 1.058805 \\ \mathrm{H} & -3.44921 & 1.008669 & 1.912843 \\ \mathrm{C} & 2.192763 & 2.129269 & 0.745592 \\ \mathrm{H} & 1.427552 & 2.22794 & 1.521276 \\ \mathrm{H} & 2.907804 & 2.939211 & 0.890464 \\ \mathrm{C} & -2.47784 & -0.1761 & 0.423484 \\ \mathrm{C} & -2.89261 & -1.03228 & -0.74726 \\ \mathrm{H} & -2.66529 & -0.48817 & -1.67227 \\ \mathrm{C} & -0.61506 & -2.11531 & -0.62303 \\ \mathrm{H} & -0.08313 & -3.06908 & -0.61343 \\ \mathrm{H} & -0.25094 & -1.55043 & -1.48394 \\ \mathrm{C} & 3.784826 & -3.2825 & -0.54003 \\ \mathrm{H} & 3.062535 & -3.79871 & -1.16418 \\ \mathrm{H} & 4.563005 & -3.88375 & -0.08651 \\ \mathrm{C} & 2.682284 & -1.0848 & -0.95403 \\ & & & \\ & & & \end{array}$




$\begin{array}{lrrr}\text { H } & 1.992273 & -1.68671 & -1.54854 \\ \mathrm{H} & 3.172315 & -0.39177 & -1.64675 \\ \mathrm{C} & 3.714649 & -1.97517 & -0.33973 \\ \mathrm{H} & 4.452107 & -1.49768 & 0.298994 \\ \mathrm{C} & -4.4149 & -1.16957 & -0.55326 \\ \mathrm{H} & -4.96344 & -1.13924 & -1.49449 \\ \mathrm{H} & -4.62671 & -2.13417 & -0.08528 \\ \mathrm{C} & -4.82201 & -0.03588 & 0.411067 \\ \mathrm{H} & -5.24384 & 0.821619 & -0.12424 \\ \mathrm{H} & -5.57808 & -0.35424 & 1.131756 \\ \mathrm{C} & -0.32541 & -1.35784 & 0.681641 \\ \mathrm{H} & -0.72006 & -1.94561 & 1.51288 \\ \mathrm{C} & 1.54617 & 2.144944 & -0.64158 \\ \mathrm{H} & 2.311312 & 2.317665 & -1.40243 \\ \mathrm{~N} & -0.43559 & 0.903036 & -0.28025 \\ \mathrm{O} & 1.672242 & -1.65276 & 1.926909\end{array}$

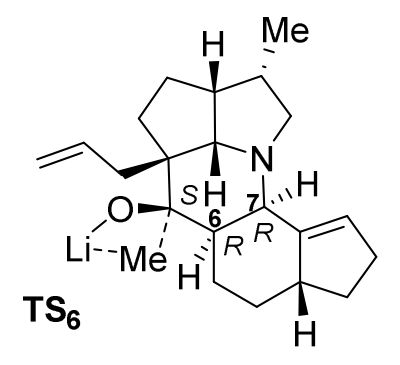

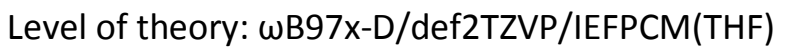
Total Energy $=-995.15381777 \mathrm{au}$ RMS Gradient Norm $=0.00000463$ au Imaginary Frequencies $=1$ Dipole Moment $=2.72$ Debye

\begin{tabular}{llll} 
Symbol & $\mathbf{X}$ & $\mathbf{Y}$ & $\mathbf{Z}$ \\
\hline $\mathrm{C}$ & -0.67704 & 2.350021 & 0.041235 \\
$\mathrm{H}$ & -0.49595 & 2.567624 & 1.109303 \\
$\mathrm{H}$ & -1.69027 & 2.697418 & -0.18246 \\
$\mathrm{C}$ & 0.938233 & -1.32913 & 0.786801
\end{tabular}




\begin{tabular}{lrrr}
$\mathrm{C}$ & -2.41198 & -2.29723 & -0.86417 \\
$\mathrm{H}$ & -2.82776 & -2.85194 & -0.01553 \\
$\mathrm{H}$ & -2.62224 & -2.88159 & -1.76361 \\
$\mathrm{C}$ & 1.720185 & -0.35646 & -0.10733 \\
$\mathrm{C}$ & 0.743214 & 4.43659 & -0.39328 \\
$\mathrm{H}$ & 1.135328 & 4.443886 & 0.625742 \\
$\mathrm{H}$ & -0.1278 & 5.094709 & -0.41953 \\
$\mathrm{H}$ & 1.503854 & 4.863154 & -1.05036 \\
$\mathrm{C}$ & 0.781488 & 0.652742 & -0.83597 \\
$\mathrm{H}$ & 0.637431 & 0.299672 & -1.86502 \\
$\mathrm{C}$ & -1.22342 & 0.024864 & 0.622197 \\
$\mathrm{H}$ & -1.1839 & 0.399344 & 1.649368 \\
$\mathrm{C}$ & 2.789246 & 0.510281 & 0.603014 \\
$\mathrm{H}$ & 2.920085 & 0.210223 & 1.640612 \\
$\mathrm{H}$ & 3.747198 & 0.375818 & 0.097863 \\
$\mathrm{C}$ & 0.371921 & 3.03088 & -0.83258 \\
$\mathrm{H}$ & -0.02134 & 3.075483 & -1.85302 \\
$\mathrm{C}$ & -3.70703 & 0.537992 & 0.787863 \\
$\mathrm{H}$ & -3.6383 & 1.210547 & 1.635546 \\
$\mathrm{C}$ & 2.33808 & 1.963271 & 0.468634 \\
$\mathrm{H}$ & 1.722056 & 2.24767 & 1.322619 \\
$\mathrm{H}$ & 3.180293 & 2.655947 & 0.437754 \\
$\mathrm{C}$ & -2.67158 & -0.07608 & 0.230027 \\
$\mathrm{C}$ & -3.08633 & -0.93025 & -0.93996 \\
$\mathrm{H}$ & -2.75896 & -0.44266 & -1.86734 \\
$\mathrm{C}$ & -0.90623 & -2.14442 & -0.67934 \\
$\mathrm{H}$ & -0.42468 & -3.12263 & -0.62894 \\
$\mathrm{H}$ & -0.49884 & -1.63062 & -1.5541 \\
$\mathrm{C}$ & 4.826169 & -1.81738 & -1.27505 \\
$\mathrm{H}$ & 5.057653 & -0.99885 & -1.94968 \\
$\mathrm{H}$ & 5.649336 & -2.456 & -0.97754 \\
& & & \\
\hline & &
\end{tabular}




$\begin{array}{lrrr}\mathrm{C} & 2.411053 & -1.197 & -1.23134 \\ \mathrm{H} & 1.664808 & -1.84693 & -1.69546 \\ \mathrm{H} & 2.728042 & -0.48402 & -1.99824 \\ \mathrm{C} & 3.592051 & -2.02717 & -0.83754 \\ \mathrm{H} & 3.395058 & -2.84875 & -0.1599 \\ \mathrm{C} & -4.62339 & -0.94241 & -0.84133 \\ \mathrm{H} & -5.10883 & -0.89745 & -1.81626 \\ \mathrm{H} & -4.93999 & -1.87359 & -0.36438 \\ \mathrm{C} & -4.99604 & 0.247617 & 0.067073 \\ \mathrm{H} & -5.31279 & 1.119425 & -0.51586 \\ \mathrm{H} & -5.81974 & 0.011927 & 0.744506 \\ \mathrm{C} & -0.56997 & -1.35755 & 0.607134 \\ \mathrm{H} & -0.97221 & -1.92467 & 1.44884 \\ \mathrm{C} & 1.507259 & 2.004192 & -0.81724 \\ \mathrm{H} & 2.164198 & 2.112872 & -1.68347 \\ \mathrm{~N} & -0.52521 & 0.943499 & -0.25386 \\ \mathrm{O} & 1.498572 & -2.37097 & 1.190244 \\ \mathrm{Li} & 1.5935 & -1.88342 & 2.979472 \\ \mathrm{C} & 0.802092 & 0.030785 & 2.981562 \\ \mathrm{H} & 1.626169 & 0.225583 & 3.689804 \\ \mathrm{H} & 0.627246 & 0.96547 & 2.455523 \\ \mathrm{H} & -0.09567 & -0.18848 & 3.57751\end{array}$

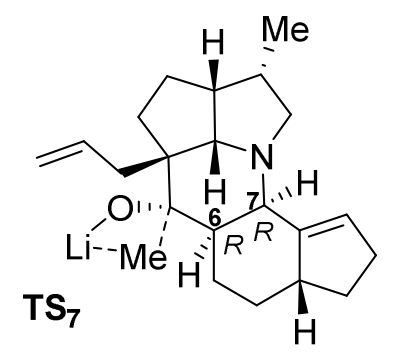

Level of theory: $\omega B 97 x-D /$ def2TZVP/IEFPCM(THF)

Total Energy $=-995.14927407 \mathrm{au}$

RMS Gradient Norm $=0.00000431 \mathrm{au}$

Imaginary Frequencies $=1$

Dipole Moment $=2.46$ Debye 


\begin{tabular}{lrrr} 
Symbol & \multicolumn{1}{l}{ X } & \multicolumn{1}{l}{$Z$} \\
\hline $\mathrm{C}$ & -0.98662 & 2.239195 & 0.010483 \\
$\mathrm{H}$ & -0.81378 & 2.457252 & 1.080826 \\
$\mathrm{H}$ & -2.04322 & 2.434153 & -0.18863 \\
$\mathrm{C}$ & 1.067904 & -1.31661 & 0.52752 \\
$\mathrm{C}$ & -2.48342 & -2.15445 & -1.03695 \\
$\mathrm{H}$ & -2.7375 & -2.9166 & -0.29083 \\
$\mathrm{H}$ & -2.83727 & -2.5272 & -2.00159 \\
$\mathrm{C}$ & 1.71923 & -0.00675 & 0.036029 \\
$\mathrm{C}$ & 0.022359 & 4.545714 & -0.47702 \\
$\mathrm{H}$ & 0.320779 & 4.6867 & 0.563235 \\
$\mathrm{H}$ & -0.94833 & 5.028086 & -0.60841 \\
$\mathrm{H}$ & 0.742874 & 5.069721 & -1.10823 \\
$\mathrm{C}$ & 0.748172 & 0.808877 & -0.83965 \\
$\mathrm{H}$ & 0.74249 & 0.419683 & -1.86393 \\
$\mathrm{C}$ & -1.08098 & -0.12826 & 0.621929 \\
$\mathrm{H}$ & -0.80807 & 0.143464 & 1.656196 \\
$\mathrm{C}$ & 1.939567 & 0.973757 & 1.216883 \\
$\mathrm{H}$ & 1.096542 & 0.925904 & 1.906414 \\
$\mathrm{H}$ & 2.83122 & 0.740677 & 1.792198 \\
$\mathrm{C}$ & -0.05798 & 3.076776 & -0.85205 \\
$\mathrm{H}$ & -0.41565 & 3.007984 & -1.88458 \\
$\mathrm{C}$ & -3.4736 & 0.149483 & 1.442315 \\
$\mathrm{H}$ & -3.24078 & 0.635651 & 2.38284 \\
$\mathrm{C}$ & 2.013802 & 2.360515 & 0.563288 \\
$\mathrm{H}$ & 1.601192 & 3.120398 & 1.226742 \\
$\mathrm{H}$ & 3.050249 & 2.643744 & 0.378024 \\
$\mathrm{C}$ & -2.5722 & -0.24504 & 0.553773 \\
$\mathrm{C}$ & -3.20137 & -0.85904 & -0.66733 \\
$\mathrm{H}$ & -3.09749 & -0.15807 & -1.50447 \\
$\mathrm{C}$ & -0.96378 & -1.99078 & -1.07145 \\
& & & \\
& & &
\end{tabular}




$\begin{array}{lrrr}\text { H } & -0.50778 & -2.95111 & -1.29162 \\ \text { H } & -0.68054 & -1.31047 & -1.87673 \\ \text { C } & 4.508662 & -1.23874 & 1.07617 \\ \text { H } & 3.799979 & -1.14722 & 1.887793 \\ \text { H } & 5.473116 & -1.67754 & 1.302785 \\ \text { C } & 3.028713 & -0.17507 & -0.75287 \\ \text { H } & 2.803233 & -0.67944 & -1.69106 \\ \text { H } & 3.373661 & 0.822965 & -1.04694 \\ \text { C } & 4.233062 & -0.85193 & -0.16445 \\ \text { H } & 5.014055 & -0.99883 & -0.90809 \\ \text { C } & -4.67934 & -1.0133 & -0.25875 \\ \text { H } & -5.36749 & -0.78853 & -1.07357 \\ \text { H } & -4.85864 & -2.04929 & 0.039699 \\ \text { C } & -4.87978 & -0.08938 & 0.96141 \\ \text { H } & -5.35108 & 0.858869 & 0.680611 \\ \text { H } & -5.5198 & -0.53931 & 1.723514 \\ \text { C } & -0.43561 & -1.48632 & 0.274216 \\ \text { H } & -0.75243 & -2.1988 & 1.040024 \\ \text { C } & 1.240356 & 2.266448 & -0.77537 \\ \text { H } & 1.893126 & 2.511539 & -1.61328 \\ \text { N } & -0.62979 & 0.874052 & -0.3246 \\ \text { O } & 1.526572 & -1.85058 & 1.559796 \\ \text { Li } & 2.582739 & -3.09721 & 0.620342 \\ \text { C } & 1.923691 & -2.91945 & -1.3347 \\ \text { H } & 1.451114 & -3.91132 & -1.38008 \\ \text { H } & 1.465909 & -2.30182 & -2.10568 \\ \text { H } & 2.982929 & -3.04728 & -1.61087\end{array}$




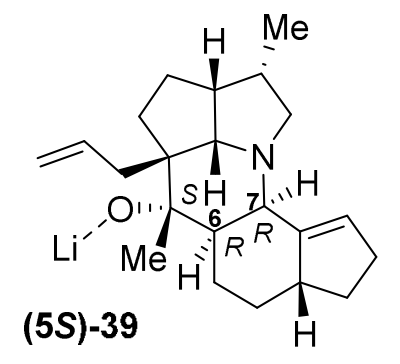

Level of theory: $\omega B 97 x-D /$ def2TZVP/IEFPCM(THF)

Total Energy $=-995.21630160 \mathrm{au}$

RMS Gradient Norm $=0.00009250 \mathrm{au}$

Imaginary Frequencies $=0$

Dipole Moment $=7.82$ Debye

\begin{tabular}{|c|c|c|c|}
\hline Symbol & $x$ & $\mathbf{Y}$ & Z \\
\hline $\mathrm{C}$ & -0.83133 & 2.371145 & -0.08939 \\
\hline $\mathrm{H}$ & -0.6806 & 2.628889 & 0.976606 \\
\hline $\mathrm{H}$ & -1.86257 & 2.645995 & -0.33309 \\
\hline C & 1.054947 & -0.9155 & 1.117546 \\
\hline C & -2.15725 & -2.43174 & -0.68085 \\
\hline $\mathrm{H}$ & -2.51211 & -2.97459 & 0.203124 \\
\hline $\mathrm{H}$ & -2.31677 & -3.08607 & -1.5425 \\
\hline C & 1.775123 & -0.16768 & -0.08111 \\
\hline C & 0.449575 & 4.538476 & -0.53975 \\
\hline $\mathrm{H}$ & 0.816091 & 4.589272 & 0.487951 \\
\hline $\mathrm{H}$ & -0.46197 & 5.137366 & -0.59941 \\
\hline $\mathrm{H}$ & 1.197099 & 5.003403 & -1.18604 \\
\hline C & 0.768228 & 0.751427 & -0.86285 \\
\hline $\mathrm{H}$ & 0.670599 & 0.35529 & -1.88122 \\
\hline C & -1.19315 & 0.071982 & 0.630258 \\
\hline $\mathrm{H}$ & -1.15166 & 0.533937 & 1.625173 \\
\hline C & 2.845095 & 0.822264 & 0.445722 \\
\hline $\mathrm{H}$ & 3.194579 & 0.544787 & 1.43864 \\
\hline $\mathrm{H}$ & 3.707078 & 0.825291 & -0.22855 \\
\hline C & 0.185555 & 3.101834 & -0.95662 \\
\hline $\mathrm{H}$ & -0.18455 & 3.097721 & -1.9869 \\
\hline
\end{tabular}




$\begin{array}{lrrr}\mathrm{C} & -3.71821 & 0.375338 & 0.796189 \\ \mathrm{H} & -3.71123 & 1.114168 & 1.590128 \\ \mathrm{C} & 2.205673 & 2.20151 & 0.402353 \\ \mathrm{H} & 1.542519 & 2.320685 & 1.26206 \\ \mathrm{H} & 2.933776 & 3.015335 & 0.415191 \\ \mathrm{C} & -2.6302 & -0.1753 & 0.271851 \\ \mathrm{C} & -2.97005 & -1.1479 & -0.82921 \\ \mathrm{H} & -2.70517 & -0.69194 & -1.79193 \\ \mathrm{C} & -0.67118 & -2.11617 & -0.51735 \\ \mathrm{H} & -0.10478 & -3.04657 & -0.44122 \\ \mathrm{H} & -0.33002 & -1.61212 & -1.42464 \\ \mathrm{C} & 4.04904 & -3.00574 & -1.17906 \\ \mathrm{H} & 3.36707 & -3.61824 & -1.76074 \\ \mathrm{H} & 5.009452 & -3.44061 & -0.92995 \\ \mathrm{C} & 2.434395 & -1.09191 & -1.13906 \\ \mathrm{H} & 1.726842 & -1.83383 & -1.50981 \\ \mathrm{H} & 2.664791 & -0.4447 & -1.99574 \\ \mathrm{C} & 3.716404 & -1.78534 & -0.78306 \\ \mathrm{H} & 4.434004 & -1.22006 & -0.1951 \\ \mathrm{C} & -4.49667 & -1.30607 & -0.70349 \\ \mathrm{H} & -4.99482 & -1.38875 & -1.66986 \\ \mathrm{H} & -4.71218 & -2.22296 & -0.14836 \\ \mathrm{C} & -4.97832 & -0.09061 & 0.115388 \\ \mathrm{H} & -5.38046 & 0.698538 & -0.52997 \\ \mathrm{H} & -5.77364 & -0.35153 & 0.817507 \\ \mathrm{C} & -0.41753 & -1.24052 & 0.717101 \\ \mathrm{H} & -0.83028 & -1.78088 & 1.577862 \\ \mathrm{C} & 1.385011 & 2.158235 & -0.88656 \\ \mathrm{H} & 2.050607 & 2.284867 & -1.74498 \\ \mathrm{~N} & -0.58542 & 0.969351 & -0.33453 \\ \mathrm{O} & 1.02946 & -0.12056 & 2.228025\end{array}$




$\begin{array}{lrrr}\text { C } & 1.766494 & -2.22705 & 1.483643 \\ \text { H } & 2.798934 & -2.02202 & 1.773912 \\ \text { H } & 1.250521 & -2.65098 & 2.348557 \\ \text { H } & 1.78529 & -2.97411 & 0.689857 \\ \text { Li } & 0.998627 & 0.86649 & 3.606902\end{array}$

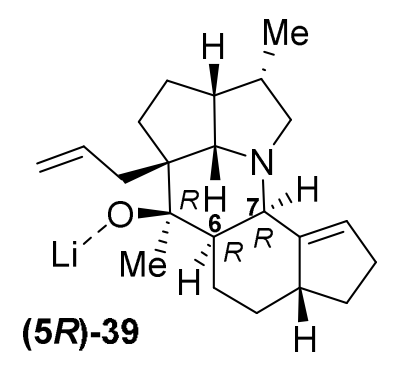

Level of theory: $\omega B$ 97x-D/def2TZVP/IEFPCM(THF)

Total Energy $=-995.21964154 \mathrm{au}$

RMS Gradient Norm $=0.00000464$ au

Imaginary Frequencies $=0$

Dipole Moment $=6.85$ Debye

\begin{tabular}{lrrl} 
Symbol & \multicolumn{1}{l}{$\mathbf{X}$} & $\mathrm{Z}$ \\
\hline $\mathrm{C}$ & -0.95546 & 2.400504 & 0.088172 \\
$\mathrm{H}$ & -0.76417 & 2.649301 & 1.149825 \\
$\mathrm{H}$ & -2.01017 & 2.625761 & -0.09911 \\
$\mathrm{C}$ & 1.108228 & -0.89995 & 1.101937 \\
$\mathrm{C}$ & -2.03807 & -2.4824 & -0.69544 \\
$\mathrm{H}$ & -2.36891 & -3.05528 & 0.178861 \\
$\mathrm{H}$ & -2.1568 & -3.13186 & -1.56748 \\
$\mathrm{C}$ & 1.748915 & -0.00686 & -0.04511 \\
$\mathrm{C}$ & 0.19146 & 4.636055 & -0.40424 \\
$\mathrm{H}$ & 0.605879 & 4.700131 & 0.604108 \\
$\mathrm{H}$ & -0.75069 & 5.188377 & -0.41289 \\
$\mathrm{H}$ & 0.880846 & 5.141417 & -1.08386 \\
$\mathrm{C}$ & 0.68269 & 0.870283 & -0.79045 \\
$\mathrm{H}$ & 0.569104 & 0.473863 & -1.80741 \\
$\mathrm{C}$ & -1.23101 & 0.040516 & 0.693971
\end{tabular}




\begin{tabular}{lrrr} 
H & -1.27183 & 0.463241 & 1.708423 \\
$\mathrm{C}$ & 2.795478 & 1.033252 & 0.448127 \\
$\mathrm{H}$ & 3.222298 & 0.791786 & 1.42011 \\
$\mathrm{H}$ & 3.631605 & 1.057164 & -0.25748 \\
$\mathrm{C}$ & -0.02299 & 3.191283 & -0.82129 \\
$\mathrm{H}$ & -0.44865 & 3.176568 & -1.82958 \\
$\mathrm{C}$ & -3.76911 & 0.205168 & 0.811825 \\
$\mathrm{H}$ & -3.81336 & 0.921334 & 1.624961 \\
$\mathrm{C}$ & 2.115659 & 2.395553 & 0.410441 \\
$\mathrm{H}$ & 1.50768 & 2.549857 & 1.304992 \\
$\mathrm{H}$ & 2.827508 & 3.221178 & 0.35958 \\
$\mathrm{C}$ & -2.64579 & -0.27584 & 0.293569 \\
$\mathrm{C}$ & -2.91611 & -1.24075 & -0.83311 \\
$\mathrm{H}$ & -2.6608 & -0.75325 & -1.78327 \\
$\mathrm{C}$ & -0.57675 & -2.07939 & -0.52042 \\
$\mathrm{H}$ & 0.063058 & -2.96065 & -0.45194 \\
$\mathrm{H}$ & -0.27425 & -1.53147 & -1.41604 \\
$\mathrm{C}$ & 4.14491 & -2.72179 & -1.24205 \\
$\mathrm{H}$ & 3.566145 & -3.26584 & -1.9821 \\
$\mathrm{H}$ & 5.10784 & -3.13545 & -0.96635 \\
$\mathrm{C}$ & 2.433843 & -0.89263 & -1.10966 \\
$\mathrm{H}$ & 1.745898 & -1.63068 & -1.51743 \\
$\mathrm{H}$ & 2.691777 & -0.22411 & -1.94202 \\
$\mathrm{C}$ & 3.700819 & -1.58408 & -0.71247 \\
$\mathrm{H}$ & 4.328504 & -1.07636 & 0.01437 \\
$\mathrm{C}$ & -4.43428 & -1.4799 & -0.73661 \\
$\mathrm{H}$ & -4.91145 & -1.5675 & -1.71307 \\
$\mathrm{H}$ & -4.61039 & -2.41835 & -0.20416 \\
$\mathrm{C}$ & -4.99164 & -0.30886 & 0.098574 \\
$\mathrm{H}$ & -5.42238 & 0.472986 & -0.53703 \\
$\mathrm{H}$ & -5.78395 & -0.62475 & 0.781061 \\
& & & \\
\hline
\end{tabular}




$\begin{array}{lrrr}\mathrm{C} & -0.3732 & -1.22584 & 0.735212 \\ \mathrm{H} & -0.74049 & -1.8267 & 1.575473 \\ \mathrm{C} & 1.225461 & 2.30802 & -0.82984 \\ \mathrm{H} & 1.832893 & 2.475576 & -1.72308 \\ \mathrm{~N} & -0.65149 & 1.020476 & -0.20307 \\ \mathrm{O} & 1.807859 & -2.05306 & 1.286734 \\ \mathrm{C} & 1.078102 & -0.13155 & 2.443887 \\ \mathrm{H} & 0.444047 & -0.67459 & 3.147988 \\ \mathrm{H} & 2.083014 & -0.10477 & 2.863467 \\ \mathrm{H} & 0.706646 & 0.892426 & 2.376434 \\ \mathrm{Li} & 2.88138 & -3.34769 & 1.000702\end{array}$

Level of theory: $\omega B$ 97x-D/def2TZVP/IEFPCM(THF)

Total Energy $=-47.44575744 \mathrm{au}$

MeLi RMS Gradient Norm $=0.00004118$ au

Imaginary Frequencies $=0$

Dipole Moment $=7.53$ Debye

\begin{tabular}{lrrl} 
Symbol & \multicolumn{1}{l}{ X } & \multicolumn{1}{l}{$\mathbf{Z}$} \\
\hline Li & -1.68691 & 0.000811 & -0.00013 \\
C & 0.41769 & 0.00046 & $-6.9 \mathrm{E}-05$ \\
H & 0.849467 & -0.56219 & -0.84254 \\
H & 0.856426 & 1.008512 & -0.06362 \\
H & 0.8487 & -0.45151 & 0.906958
\end{tabular}




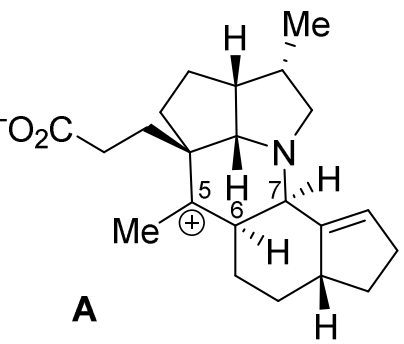

Level of theory: $\omega B 97 x-D /$ def2TZVP

Total Energy $=-1062.17950605 \mathrm{au}$

RMS Gradient Norm $=0.00000849$ au

Imaginary Frequencies $=0$

Dipole Moment $=18.78$ Debye

\begin{tabular}{lrrr} 
Symbol & \multicolumn{1}{l}{ X } & \multicolumn{1}{l}{ Z } \\
\hline C & -0.1423 & 2.633271 & 0.813318 \\
H & 0.308432 & 2.373572 & 1.782349 \\
H & -0.97299 & 3.316636 & 0.994679 \\
C & -0.11128 & -1.24381 & 0.32854 \\
C & -3.50331 & -1.22778 & 1.570692 \\
H & -3.9007 & -0.34467 & 2.080877 \\
H & -4.14204 & -2.06375 & 1.859918 \\
C & 1.060801 & -0.53299 & -0.10298 \\
C & 1.804749 & 4.258445 & 0.43161 \\
H & 2.28509 & 3.933781 & 1.354714 \\
H & 1.234931 & 5.162268 & 0.655089 \\
H & 2.589309 & 4.525167 & -0.27758 \\
C & 0.387578 & 0.913831 & -0.73477 \\
H & 0.029258 & 0.616823 & -1.72047 \\
C & -1.61533 & 0.642566 & 0.621014 \\
H & -2.09345 & 1.175145 & 1.447801 \\
C & 1.98515 & 0.100817 & 0.95149 \\
H & 1.416642 & 0.426882 & 1.822097 \\
H & 2.720595 & -0.628 & 1.29301 \\
C & 0.895248 & 3.191173 & -0.14897 \\
H & 0.344144 & 3.631609 & -0.98595
\end{tabular}




\begin{tabular}{lrrr} 
C & -3.17824 & 0.814297 & -1.37825 \\
H & -2.74006 & 1.687883 & -1.84318 \\
C & 2.634606 & 1.291536 & 0.254937 \\
H & 3.014609 & 2.006573 & 0.982333 \\
H & 3.491966 & 0.932221 & -0.31509 \\
C & -2.69996 & 0.197905 & -0.30611 \\
C & -3.54737 & -0.99514 & 0.070024 \\
H & -3.14222 & -1.88656 & -0.4212 \\
C & -2.0629 & -1.51204 & 2.041815 \\
H & -2.00347 & -1.33684 & 3.116006 \\
H & -1.84367 & -2.5712 & 1.90803 \\
$\mathrm{C}$ & 1.829403 & -1.16206 & -1.27269 \\
H & 1.120956 & -1.44958 & -2.05372 \\
H & 2.486507 & -0.41269 & -1.71651 \\
$\mathrm{C}$ & 2.742529 & -2.31852 & -0.86834 \\
$\mathrm{C}$ & -4.87867 & -0.65804 & -0.60863 \\
$\mathrm{H}$ & -5.47056 & -1.53997 & -0.85341 \\
$\mathrm{H}$ & -5.47357 & -0.02385 & 0.054955 \\
$\mathrm{C}$ & -4.45289 & 0.157184 & -1.847 \\
$\mathrm{H}$ & -4.26642 & -0.49481 & -2.70776 \\
$\mathrm{H}$ & -5.20644 & 0.881509 & -2.1583 \\
$\mathrm{C}$ & -0.95094 & -0.67117 & 1.372115 \\
$\mathrm{C}$ & 1.570219 & 1.906814 & -0.67766 \\
$\mathrm{H}$ & 1.987138 & 2.077041 & -1.66866 \\
$\mathrm{~N}$ & -0.61 & 1.45896 & 0.091995 \\
$\mathrm{C}$ & -0.59364 & -2.38596 & -0.47954 \\
$\mathrm{H}$ & -1.00921 & -1.98486 & -1.41452 \\
$\mathrm{H}$ & 0.232969 & -3.03473 & -0.76176 \\
$\mathrm{H}$ & -1.36851 & -2.96435 & 0.011071 \\
$\mathrm{H}$ & 2.264554 & -3.00988 & -0.17 \\
$\mathrm{H}$ & -0.33387 & -0.20927 & 2.136411 \\
& & & \\
\hline
\end{tabular}




$\begin{array}{lrrr}C & 4.073505 & -1.80057 & -0.22605 \\ \mathrm{O} & 4.405729 & -2.34343 & 0.837899 \\ \mathrm{O} & 4.621554 & -0.87211 & -0.85483 \\ \mathrm{H} & 3.01008 & -2.87913 & -1.76713\end{array}$

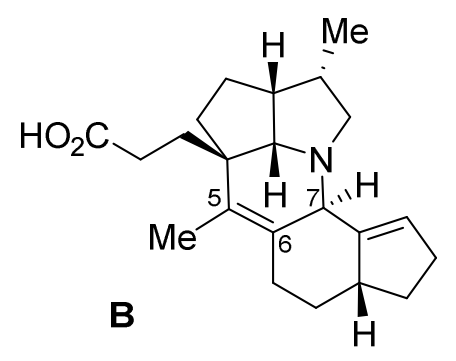

Level of theory: $\omega B$ 97x-D/def2TZVP

Total Energy $=-1062.27261983 \mathrm{au}$

RMS Gradient Norm $=0.00000346$ au

Imaginary Frequencies $=0$

Dipole Moment $=1.67$ Debye

\begin{tabular}{lrrl} 
Symbol & \multicolumn{1}{l}{$\mathbf{X}$} & $\mathrm{Z}$ \\
\hline $\mathrm{C}$ & -1.33747 & 2.393678 & 0.311247 \\
$\mathrm{H}$ & -1.10044 & 2.461709 & 1.390687 \\
$\mathrm{H}$ & -2.39932 & 2.63179 & 0.201085 \\
$\mathrm{C}$ & 0.640498 & -1.24133 & 0.148712 \\
$\mathrm{C}$ & -2.58068 & -2.72506 & -0.30033 \\
$\mathrm{H}$ & -3.37852 & -3.32732 & 0.142069 \\
$\mathrm{H}$ & -2.17617 & -3.29633 & -1.13849 \\
$\mathrm{C}$ & 1.2944 & 0.100536 & -0.16638 \\
$\mathrm{C}$ & -0.27144 & 4.715053 & 0.108948 \\
$\mathrm{H}$ & 0.098523 & 4.683402 & 1.135213 \\
$\mathrm{H}$ & -1.22817 & 5.24091 & 0.122324 \\
$\mathrm{H}$ & 0.426996 & 5.311368 & -0.48161 \\
$\mathrm{C}$ & 0.284623 & 1.052368 & -0.83889 \\
$\mathrm{H}$ & 0.190307 & 0.793771 & -1.90029 \\
$\mathrm{C}$ & -1.49351 & -0.02254 & 0.588133 \\
$\mathrm{H}$ & -1.44971 & 0.264048 & 1.656619
\end{tabular}




$\begin{array}{lrrr}C & 1.649914 & 0.907232 & 1.097926 \\ \mathrm{H} & 0.83436 & 0.799499 & 1.815857 \\ \mathrm{H} & 2.551957 & 0.553558 & 1.597961 \\ \mathrm{C} & -0.4342 & 3.325151 & -0.47999 \\ \mathrm{H} & -0.86198 & 3.4271 & -1.48245 \\ \mathrm{C} & -4.04619 & 0.032096 & 0.84415 \\ \mathrm{H} & -4.10163 & 0.701462 & 1.694917 \\ \mathrm{C} & 1.742113 & 2.36465 & 0.626506 \\ \mathrm{H} & 1.448168 & 3.052591 & 1.419309 \\ \mathrm{H} & 2.768494 & 2.624056 & 0.361383 \\ \mathrm{C} & -2.92306 & -0.35244 & 0.256432 \\ \mathrm{C} & -3.15849 & -1.38487 & -0.80883 \\ \mathrm{H} & -2.62947 & -1.10538 & -1.7237 \\ \mathrm{C} & -1.47975 & -2.50634 & 0.759282 \\ \mathrm{H} & -1.9621 & -2.35265 & 1.73052 \\ \mathrm{H} & -0.87802 & -3.40582 & 0.856177 \\ \mathrm{C} & 2.489185 & 0.001127 & -1.16395 \\ \mathrm{H} & 2.350326 & -0.85713 & -1.8222 \\ \mathrm{H} & 2.434522 & 0.869611 & -1.82116 \\ \mathrm{C} & 3.934801 & 0.003164 & -0.63913 \\ \mathrm{C} & -4.68435 & -1.33601 & -1.01328 \\ \mathrm{H} & -4.91353 & -0.73914 & -1.89717 \\ \mathrm{H} & -5.11585 & -2.32533 & -1.17216 \\ \mathrm{C} & -5.25455 & -0.63322 & 0.240451 \\ \mathrm{H} & -6.048 & 0.073695 & -0.01288 \\ \mathrm{H} & -5.69079 & -1.34488 & 0.949495 \\ \mathrm{C} & -0.65799 & -1.28082 & 0.454575 \\ \mathrm{C} & 0.835232 & 2.477047 & -0.62612 \\ \mathrm{H} & 1.417182 & 2.82189 & -1.48144 \\ \mathrm{~N} & -1.03315 & 1.082703 & -0.21792 \\ \mathrm{C} & 1.517466 & -2.45945 & 0.180135\end{array}$




$\begin{array}{lrrr}\mathrm{H} & 2.037688 & -2.63096 & -0.76168 \\ \mathrm{H} & 2.283213 & -2.34234 & 0.951488 \\ \mathrm{H} & 0.965767 & -3.36498 & 0.41355 \\ \mathrm{H} & 4.582654 & 0.226163 & -1.489 \\ \mathrm{H} & 4.082326 & 0.787525 & 0.100277 \\ \mathrm{C} & 4.44517 & -1.30341 & -0.09778 \\ \mathrm{O} & 4.673824 & -2.27994 & -0.75748 \\ \mathrm{O} & 4.655417 & -1.27333 & 1.232169 \\ \mathrm{H} & 4.988864 & -2.14436 & 1.482345\end{array}$

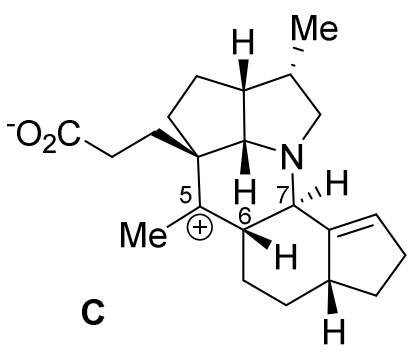

Level of theory: $\omega B 97 x-D /$ def2TZVP

Total Energy $=-1062.17703423 \mathrm{au}$

RMS Gradient Norm $=0.00000468 \mathrm{au}$

Imaginary Frequencies $=0$

Dipole Moment $=16.15$ Debye

\begin{tabular}{lrrr} 
Symbol & X & \multicolumn{1}{l}{ Y } \\
\hline C & -0.17004 & 2.300412 & 0.345661 \\
H & -0.09884 & 2.275118 & 1.45098 \\
H & -1.00077 & 2.96094 & 0.087045 \\
C & 0.015189 & -1.42114 & 1.124601 \\
C & -3.3891 & -1.85568 & -0.69355 \\
H & -4.38605 & -2.0659 & -0.30169 \\
H & -3.23893 & -2.51979 & -1.54641 \\
C & 1.210446 & -0.72814 & 0.723548 \\
C & 1.774829 & 3.957595 & 0.382411 \\
H & 1.871559 & 3.850276 & 1.464735 \\
H & 1.160594 & 4.840434 & 0.19458
\end{tabular}




$\begin{array}{lrrr}\text { H } & 2.768096 & 4.148085 & -0.02665 \\ \text { C } & 0.939142 & 0.315653 & -0.39453 \\ \text { H } & 1.004172 & -0.14788 & -1.37617 \\ \text { C } & -1.39145 & 0.212046 & 0.453284 \\ \text { H } & -1.37858 & 0.410797 & 1.5468 \\ \text { C } & 1.989937 & 0.016865 & 1.819395 \\ \text { H } & 1.276027 & 0.462074 & 2.520415 \\ \text { H } & 2.646533 & -0.63976 & 2.391213 \\ \text { C } & 1.155365 & 2.728829 & -0.25828 \\ \text { H } & 0.971276 & 2.949589 & -1.31318 \\ \text { C } & -3.71882 & 1.223205 & 0.509552 \\ \text { H } & -3.57781 & 1.838542 & 1.390257 \\ \text { C } & 2.758732 & 1.113711 & 1.074905 \\ \text { H } & 2.898948 & 1.986522 & 1.711287 \\ \text { H } & 3.747824 & 0.757845 & 0.790639 \\ \text { C } & -2.78824 & 0.45009 & -0.03119 \\ \text { C } & -3.31619 & -0.38311 & -1.16479 \\ \text { H } & -2.6497 & -0.32327 & -2.02813 \\ \text { C } & -2.35259 & -2.17128 & 0.403263 \\ \text { H } & -2.77959 & -1.95697 & 1.386778 \\ \text { H } & -2.10168 & -3.23356 & 0.39062 \\ \text { C } & 1.950171 & -2.0578 & 0.179142 \\ \text { H } & 1.89241 & -2.83762 & 0.943891 \\ \text { H } & 1.435954 & -2.39029 & -0.72324 \\ \text { C } & 3.411444 & -1.82436 & -0.19651 \\ \text { C } & -4.67058 & 0.283012 & -1.47274 \\ \text { H } & -4.55564 & 0.953723 & -2.32441 \\ \text { H } & -5.44106 & -0.44255 & -1.7348 \\ \text { C } & -5.03019 & 1.107978 & -0.21672 \\ \text { H } & -5.45837 & 2.079025 & -0.47187 \\ \text { H } & -5.76655 & 0.60265 & 0.416749\end{array}$




$\begin{array}{lrrr}\mathrm{C} & -1.10461 & -1.33249 & 0.205439 \\ \mathrm{C} & 1.987394 & 1.432077 & -0.22529 \\ \mathrm{H} & 2.692362 & 1.41558 & -1.05315 \\ \mathrm{~N} & -0.37798 & 0.975001 & -0.20859 \\ \mathrm{C} & -0.12035 & -2.10132 & 2.425456 \\ \mathrm{H} & 0.780894 & -2.04114 & 3.027816 \\ \mathrm{H} & -0.95339 & -1.64533 & 2.968459 \\ \mathrm{H} & -0.40506 & -3.14443 & 2.265253 \\ \mathrm{H} & -0.75168 & -1.38343 & -0.82666 \\ \mathrm{H} & 3.825387 & -2.81712 & -0.39811 \\ \mathrm{H} & 3.988249 & -1.39963 & 0.623593 \\ \mathrm{C} & 3.575191 & -0.97904 & -1.4971 \\ \mathrm{O} & 2.710171 & -1.19424 & -2.37035 \\ \mathrm{O} & 4.531728 & -0.19204 & -1.49993\end{array}$

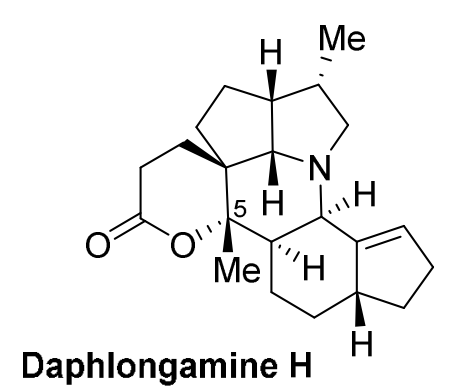

Daphlongamine $\mathrm{H}$
Level of theory: $\omega B 97 x-D /$ def2TZVP

Total Energy $=-1062.28382689 \mathrm{au}$ RMS Gradient Norm $=0.00000357 \mathrm{au}$

Imaginary Frequencies $=0$

Dipole Moment $=4.76$ Debye

\begin{tabular}{llll} 
Symbol & $\mathbf{X}$ & $\mathbf{Y}$ & $\mathbf{Z}$ \\
\hline $\mathrm{C}$ & 1.499684 & 2.121069 & 0.595981 \\
$\mathrm{H}$ & 1.965973 & 2.127646 & -0.39573 \\
$\mathrm{H}$ & 2.312337 & 2.188269 & 1.323017 \\
$\mathrm{C}$ & 0.527379 & 3.300642 & 0.709089 \\
$\mathrm{H}$ & 0.426894 & 3.569818 & 1.765407
\end{tabular}




$\begin{array}{lrrr}\text { C } & -0.79741 & 2.669445 & 0.285528 \\ \text { H } & -1.64798 & 3.238413 & 0.666377 \\ \text { C } & -0.97768 & 2.361158 & -1.21538 \\ \text { H } & -1.58128 & 3.120472 & -1.71144 \\ \text { H } & -0.00965 & 2.360461 & -1.72092 \\ \text { C } & -1.61942 & 0.956384 & -1.31156 \\ \text { H } & -1.05909 & 0.302138 & -1.97606 \\ \text { H } & -2.62233 & 1.012677 & -1.73561 \\ \text { C } & -1.68662 & 0.414313 & 0.14664 \\ \text { C } & -0.70382 & 1.286907 & 0.920201 \\ \text { H } & -0.9584 & 1.312678 & 1.9846 \\ \text { C } & 0.966959 & 4.536174 & -0.05778 \\ \text { H } & 1.939408 & 4.887579 & 0.293082 \\ \text { H } & 0.253127 & 5.352385 & 0.069125 \\ \text { H } & 1.056208 & 4.329136 & -1.12641 \\ \text { C } & -1.33665 & -1.07135 & 0.335826 \\ \text { C } & 0.138427 & -1.38272 & -0.07744 \\ \text { H } & 0.069751 & -1.8321 & -1.07087 \\ \text { C } & 0.81239 & -2.42113 & 0.838118 \\ \text { H } & 0.774436 & -2.07844 & 1.87402 \\ \text { H } & 0.242923 & -3.351 & 0.787037 \\ \text { C } & 2.274481 & -2.69885 & 0.51314 \\ \text { H } & 2.378183 & -3.10203 & -0.50021 \\ \text { H } & 2.665314 & -3.45111 & 1.203315 \\ \text { C } & 3.065708 & -1.39729 & 0.620439 \\ \text { H } & 2.973647 & -1.02837 & 1.6467 \\ \text { C } & 4.538443 & -1.45972 & 0.174831 \\ \text { H } & 5.150063 & -0.85501 & 0.845496 \\ \text { H } & 4.932523 & -2.47641 & 0.211065 \\ \text { C } & 4.579085 & -0.86237 & -1.24868 \\ \text { H } & 5.416366 & -0.17433 & -1.38595\end{array}$




$\begin{array}{lrrr}\text { H } & 4.687234 & -1.63536 & -2.01643 \\ \mathrm{C} & 3.243547 & -0.188 & -1.37892 \\ \mathrm{H} & 2.961376 & 0.393828 & -2.24915 \\ \mathrm{C} & 2.435529 & -0.45058 & -0.35979 \\ \mathrm{C} & 0.981699 & -0.09672 & -0.26814 \\ \mathrm{H} & 0.737942 & 0.323488 & -1.25182 \\ \mathrm{C} & -3.11982 & 0.637712 & 0.639495 \\ \mathrm{H} & -3.16363 & 0.583871 & 1.726595 \\ \mathrm{H} & -3.44167 & 1.645852 & 0.374819 \\ \mathrm{C} & -4.07393 & -0.38534 & 0.004632 \\ \mathrm{H} & -4.77808 & -0.77587 & 0.741367 \\ \mathrm{H} & -4.69281 & 0.071962 & -0.76694 \\ \mathrm{C} & -3.47236 & -1.60931 & -0.67566 \\ \mathrm{C} & -1.65556 & -1.53651 & 1.760354 \\ \mathrm{H} & -1.44264 & -2.59599 & 1.868948 \\ \mathrm{H} & -2.70824 & -1.40163 & 1.996464 \\ \mathrm{H} & -1.06741 & -0.98538 & 2.494005 \\ \mathrm{~N} & 0.715071 & 0.889709 & 0.780615 \\ \mathrm{O} & -2.16114 & -1.8626 & -0.55522 \\ \mathrm{O} & -4.15093 & -2.34354 & -1.3365\end{array}$

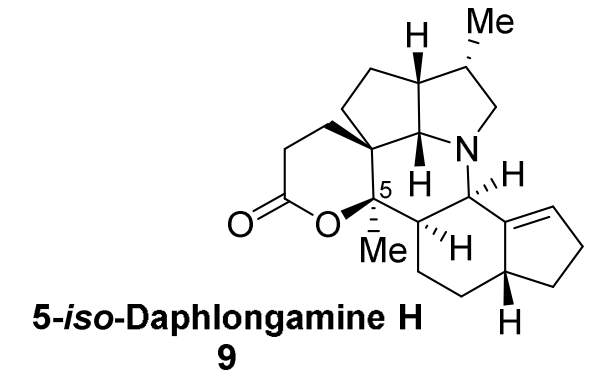

9
For NMR data, see S58-59

For X-ray data, see S133-134
Level of theory: $\omega B$ 97x-D/def2TZVP Total Energy $=-1062.29811167 \mathrm{au}$ RMS Gradient Norm $=0.00000621 \mathrm{au}$ Imaginary Frequencies $=0$ Dipole Moment $=4.95$ Debye 


\begin{tabular}{lrrr} 
Symbol & \multicolumn{1}{l}{ X } & \multicolumn{1}{l}{ Z } \\
\hline C & -1.37892 & 2.197853 & 0.087734 \\
H & -1.21761 & 2.441071 & 1.155713 \\
H & -2.45781 & 2.217899 & -0.08399 \\
C & -0.61871 & 3.174641 & -0.79603 \\
H & -0.98968 & 3.045348 & -1.81775 \\
C & 0.798182 & 2.585777 & -0.76025 \\
H & 1.371743 & 2.910248 & -1.62909 \\
C & 1.601476 & 2.837669 & 0.538015 \\
H & 2.578346 & 3.258918 & 0.299654 \\
H & 1.113795 & 3.555868 & 1.196427 \\
C & 1.742891 & 1.477554 & 1.229892 \\
H & 0.924936 & 1.345964 & 1.93877 \\
H & 2.671828 & 1.392064 & 1.796294 \\
C & 1.638656 & 0.436758 & 0.096061 \\
C & 0.54469 & 1.066485 & -0.784 \\
H & 0.603498 & 0.664511 & -1.80113 \\
C & -0.76858 & 4.63774 & -0.41988 \\
H & -1.80568 & 4.958339 & -0.53529 \\
H & -0.1519 & 5.271774 & -1.0599 \\
H & -0.48242 & 4.826786 & 0.61612 \\
C & 1.281147 & -1.01343 & 0.583724 \\
C & -0.19742 & -1.37606 & 0.31258 \\
H & -0.45624 & -2.16313 & 1.027784 \\
C & -0.51087 & -1.94116 & -1.07984 \\
H & -0.32712 & -1.19284 & -1.85322 \\
H & 0.153876 & -2.77814 & -1.28341 \\
C & -1.96452 & -2.40792 & -1.15288 \\
H & -2.10677 & -3.22935 & -0.4413 \\
H & -2.17834 & -2.81144 & -2.14554 \\
C & -2.94632 & -1.28927 & -0.80744 \\
& & & \\
& & &
\end{tabular}




$\begin{array}{lrrr}\text { H } & -2.92877 & -0.55653 & -1.62346 \\ \mathrm{C} & -4.38737 & -1.74329 & -0.50496 \\ \mathrm{H} & -5.04957 & -1.64693 & -1.36528 \\ \mathrm{H} & -4.37466 & -2.79938 & -0.22454 \\ \mathrm{C} & -4.85239 & -0.90124 & 0.701983 \\ \mathrm{H} & -5.48209 & -0.05987 & 0.392789 \\ \mathrm{H} & -5.44188 & -1.48378 & 1.413167 \\ \mathrm{C} & -3.55919 & -0.39844 & 1.284505 \\ \mathrm{H} & -3.4941 & 0.105013 & 2.242263 \\ \mathrm{C} & -2.53748 & -0.59147 & 0.463294 \\ \mathrm{C} & -1.10608 & -0.18214 & 0.632791 \\ \mathrm{H} & -0.95597 & 0.120627 & 1.68494 \\ \mathrm{C} & 2.952172 & 0.418148 & -0.70217 \\ \mathrm{H} & 2.74553 & 0.13948 & -1.739 \\ \mathrm{H} & 3.38778 & 1.417172 & -0.73054 \\ \mathrm{C} & 3.950797 & -0.57281 & -0.12827 \\ \mathrm{H} & 4.898213 & -0.56842 & -0.66341 \\ \mathrm{H} & 4.171377 & -0.35023 & 0.919012 \\ \mathrm{C} & 3.374272 & -1.95897 & -0.23801 \\ \mathrm{C} & 1.567023 & -1.24083 & 2.071276 \\ \mathrm{H} & 1.466804 & -2.30528 & 2.281553 \\ \mathrm{H} & 0.86211 & -0.70173 & 2.701869 \\ \mathrm{H} & 2.572523 & -0.93005 & 2.353078 \\ \mathrm{~N} & -0.81347 & 0.914753 & -0.2665 \\ \mathrm{O} & 2.038249 & -2.02973 & -0.11907 \\ \mathrm{O} & 4.003225 & -2.94413 & -0.49666\end{array}$




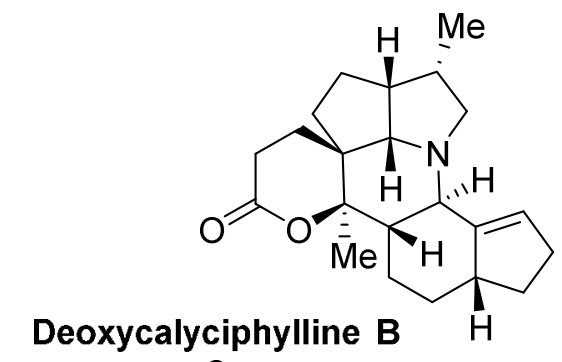

2
Level of theory: $\omega B 97 x-D / d e f 2 T Z V P$

Total Energy $=-1062.29194439 \mathrm{au}$

RMS Gradient Norm $=0.00001171 \mathrm{au}$

Imaginary Frequencies $=0$

Dipole Moment $=4.80$ Debye

For X-ray data, see S131-132

\begin{tabular}{lrrr} 
Symbol & \multicolumn{1}{l}{ X } & \multicolumn{1}{l}{ Z } \\
\hline C & -1.04338 & 2.467195 & 0.030867 \\
H & -0.91143 & 2.591974 & 1.123192 \\
H & -2.09512 & 2.662035 & -0.18966 \\
C & -0.09492 & 3.38741 & -0.7219 \\
H & -0.40774 & 3.387934 & -1.77076 \\
C & 1.21936 & 2.59726 & -0.64927 \\
H & 1.897513 & 2.902078 & -1.44688 \\
C & 1.94649 & 2.633975 & 0.717473 \\
H & 2.985903 & 2.93329 & 0.58175 \\
H & 1.508901 & 3.360836 & 1.400991 \\
C & 1.853182 & 1.219123 & 1.300691 \\
H & 0.977535 & 1.149002 & 1.94652 \\
H & 2.720507 & 0.9622 & 1.911587 \\
C & 1.693886 & 0.294341 & 0.078504 \\
C & 0.760069 & 1.136053 & -0.82048 \\
H & 0.839224 & 0.800262 & -1.85947 \\
C & -0.06433 & 4.825478 & -0.2362 \\
H & -1.03479 & 5.301329 & -0.38912 \\
H & 0.680433 & 5.407385 & -0.78275 \\
H & 0.169242 & 4.895912 & 0.827482
\end{tabular}




$\begin{array}{lrrr}\text { C } & 1.116872 & -1.13185 & 0.408005 \\ \text { C } & -0.34134 & -1.21688 & -0.05747 \\ \text { H } & -0.29611 & -1.19924 & -1.15076 \\ \text { C } & -1.10632 & -2.47383 & 0.349373 \\ \text { H } & -1.33811 & -2.42034 & 1.416949 \\ \text { H } & -0.48605 & -3.35741 & 0.199309 \\ \text { C } & -2.41598 & -2.60209 & -0.45358 \\ \text { H } & -3.14815 & -3.14875 & 0.145577 \\ \text { H } & -2.24229 & -3.1943 & -1.35469 \\ \text { C } & -3.00156 & -1.22639 & -0.84784 \\ \text { H } & -2.60409 & -0.94745 & -1.827 \\ \text { C } & -4.5385 & -1.12178 & -0.83586 \\ \text { H } & -5.02135 & -2.09336 & -0.9487 \\ \text { H } & -4.86727 & -0.50031 & -1.66992 \\ \text { C } & -4.9038 & -0.42734 & 0.495312 \\ \text { H } & -5.6978 & 0.311864 & 0.367827 \\ \text { H } & -5.26234 & -1.138 & 1.24738 \\ \text { C } & -3.60054 & 0.181757 & 0.935886 \\ \text { H } & -3.51303 & 0.830833 & 1.799769 \\ \text { C } & -2.58206 & -0.22432 & 0.191703 \\ \text { C } & -1.11484 & 0.033821 & 0.374412 \\ \text { H } & -0.94368 & 0.215511 & 1.452567 \\ \text { C } & 3.05111 & 0.160845 & -0.63451 \\ \text { H } & 2.889683 & 0.017436 & -1.70615 \\ \text { H } & 3.622854 & 1.082963 & -0.52876 \\ \text { C } & 3.856748 & -1.01668 & -0.11024 \\ \text { H } & 4.833611 & -1.09644 & -0.58287 \\ \text { H } & 4.025956 & -0.93234 & 0.966317 \\ \text { C } & 3.104123 & -2.28682 & -0.40662 \\ \text { C } & 1.268523 & -1.53441 & 1.874868 \\ \text { H } & 0.60663 & -0.95839 & 2.519365\end{array}$




$\begin{array}{lrrr}\mathrm{H} & 2.28735 & -1.39086 & 2.232188 \\ \mathrm{H} & 1.017648 & -2.58806 & 1.984576 \\ \mathrm{~N} & -0.65193 & 1.148554 & -0.41742 \\ \mathrm{O} & 1.766198 & -2.17212 & -0.37075 \\ \mathrm{O} & 3.607946 & -3.31997 & -0.74084\end{array}$

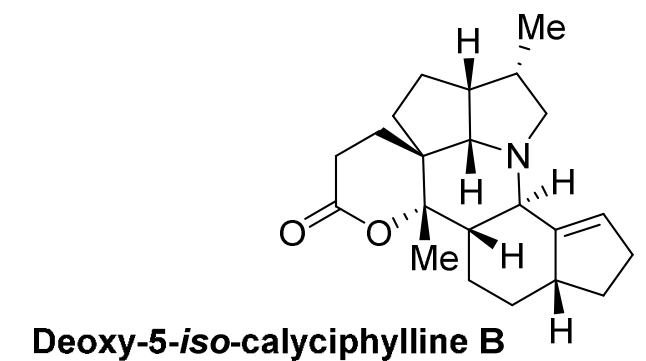

Deoxy-5-iso-calyciphylline B
Level of theory: $\omega B$ 97x-D/def2TZVP Total Energy $=-1062.27684131 \mathrm{au}$ RMS Gradient Norm $=0.00001377 \mathrm{au}$

Imaginary Frequencies $=0$

Dipole Moment $=4.43$ Debye

\begin{tabular}{lrrr} 
Symbol & X & \multicolumn{1}{l}{ Z } \\
\hline C & 1.032543 & 2.461889 & 0.036947 \\
H & 1.048045 & 2.439974 & -1.0673 \\
H & 2.039866 & 2.727653 & 0.363555 \\
C & -0.02349 & 3.44889 & 0.51308 \\
H & 0.161734 & 3.655672 & 1.572322 \\
C & -1.28964 & 2.592408 & 0.43875 \\
H & -2.08802 & 3.013939 & 1.051301 \\
C & -1.80424 & 2.272922 & -0.98715 \\
H & -2.81735 & 2.654057 & -1.1158 \\
H & -1.19851 & 2.75454 & -1.75507 \\
C & -1.76413 & 0.740632 & -1.15725 \\
H & -0.91689 & 0.436738 & -1.76334 \\
H & -2.64711 & 0.377762 & -1.68266 \\
C & -1.6841 & 0.19347 & 0.291506 \\
C & -0.81138 & 1.241459 & 0.977272
\end{tabular}




$\begin{array}{lrrr}\text { H } & -0.89392 & 1.178046 & 2.068303 \\ \mathrm{C} & -0.04284 & 4.770836 & -0.23324 \\ \mathrm{H} & 0.908002 & 5.294315 & -0.11566 \\ \mathrm{H} & -0.83055 & 5.423509 & 0.14801 \\ \mathrm{H} & -0.21088 & 4.631237 & -1.30271 \\ \mathrm{C} & -1.10274 & -1.22431 & 0.472948 \\ \mathrm{C} & 0.437494 & -1.23748 & 0.38937 \\ \mathrm{H} & 0.750154 & -1.22411 & 1.436833 \\ \mathrm{C} & 1.062055 & -2.47548 & -0.24956 \\ \mathrm{H} & 0.943158 & -2.40671 & -1.33285 \\ \mathrm{H} & 0.521612 & -3.37269 & 0.052172 \\ \mathrm{C} & 2.558868 & -2.59737 & 0.125546 \\ \mathrm{H} & 3.1225 & -2.92783 & -0.75014 \\ \mathrm{H} & 2.695292 & -3.36356 & 0.892391 \\ \mathrm{C} & 3.156136 & -1.26602 & 0.625215 \\ \mathrm{H} & 2.912346 & -1.15007 & 1.684469 \\ \mathrm{C} & 4.671893 & -1.09593 & 0.408646 \\ \mathrm{H} & 5.178519 & -2.05342 & 0.279707 \\ \mathrm{H} & 5.114018 & -0.61435 & 1.28164 \\ \mathrm{C} & 4.825394 & -0.17562 & -0.82241 \\ \mathrm{H} & 5.59424 & 0.585758 & -0.67205 \\ \mathrm{H} & 5.114204 & -0.73063 & -1.721 \\ \mathrm{C} & 3.450744 & 0.407392 & -0.99447 \\ \mathrm{H} & 3.222368 & 1.157039 & -1.74325 \\ \mathrm{C} & 2.55629 & -0.15205 & -0.1915 \\ \mathrm{C} & 1.057827 & 0.029414 & -0.19262 \\ \mathrm{H} & 0.773223 & 0.12987 & -1.25088 \\ \mathrm{C} & -3.12502 & 0.135995 & 0.80059 \\ \mathrm{H} & -3.14974 & 0.018729 & 1.883937 \\ \mathrm{H} & -3.64441 & 1.069141 & 0.58043 \\ \mathrm{C} & -3.85106 & -1.03259 & 0.10121 \\ & & & \\ & & \end{array}$




$\begin{array}{lrrr}\mathrm{H} & -4.40011 & -1.64375 & 0.820075 \\ \mathrm{H} & -4.60201 & -0.67181 & -0.5998 \\ \mathrm{C} & -3.01231 & -2.01511 & -0.72151 \\ \mathrm{C} & -1.44355 & -1.86768 & 1.826244 \\ \mathrm{H} & -2.49707 & -2.11417 & 1.936912 \\ \mathrm{H} & -1.15502 & -1.20826 & 2.645819 \\ \mathrm{H} & -0.87967 & -2.79634 & 1.922082 \\ \mathrm{~N} & 0.618358 & 1.176984 & 0.582002 \\ \mathrm{O} & -1.67832 & -2.05515 & -0.55497 \\ \mathrm{O} & -3.52481 & -2.75025 & -1.51593\end{array}$




\section{X-ray data}

General information. X-ray crystallographic data were collected from a single crystal sample, which was mounted on a loop fiber. Data were collected using a Bruker Venture diffractometer equipped with a Photon 100 CMOS Detector, a Helios MX optics and a Kappa goniometer. The crystal-to-detector distance was $4.0 \mathrm{~cm}$, and the data collection was carried out in 1024 x 1024 pixel mode.

Single crystals were kept at $100 \mathrm{~K}$ during data collection. Using Olex $2,{ }^{7}$ the structure was solved with the SIR2011 structure solution program ${ }^{8}$ using Direct Methods and refined with the XL refinement package ${ }^{9}$ using Least Squares minimization.

A table summarizing the data for each crystal is given below, together with ORTEP plots. 
Table S1. Crystal data and structure refinement for 2.

Identification code

Empirical formula

Formula weight

Temperature

Wavelength

Crystal system

Space group

Unit cell dimensions

Volume

Z

Density (calculated)

Absorption coefficient

$\mathrm{F}(000)$

Crystal size

Theta range for data collection
Compound 2

$\mathrm{C}_{22} \mathrm{H}_{31} \mathrm{~N} \mathrm{O}_{2}$

341.48

$150 \mathrm{~K}$

$1.34139 \AA$

Orthorhombic

P212121

$a=7.2033(2) \AA ; \alpha=90^{\circ}$.

$b=8.5737(2) \AA ; \beta=90^{\circ}$.

$c=29.5112(9) \AA ; \gamma=90^{\circ}$.

1822.58(9) $\AA^{3}$

4

$1.244 \mathrm{Mg} / \mathrm{m}^{3}$

$0.393 \mathrm{~mm}^{-1}$

744

$0.10 \times 0.10 \times 0.08 \mathrm{~mm}^{3}$

2.605 to $60.623^{\circ}$.
Index ranges

Reflections collected

Independent reflections

Completeness to theta $=53.594^{\circ}$

Absorption correction

equivalents

Max. and min. transmission

Refinement method

on $\mathrm{F}^{2}$

Data / restraints / parameters

Goodness-of-fit on $\mathrm{F}^{2}$

Final $R$ indices [ $1>2$ sigma(I)]

$\mathrm{R}$ indices (all data)

Absolute structure parameter

Extinction coefficient

Largest diff. peak and hole $-8<=\mathrm{h}<=9,-11<=\mathrm{k}<=11,-$

$34<=1<=38$

31099

$4151[\mathrm{R}$ (int) $=0.023]$

$99.0 \%$

Semi-empirical from

0.7519 and 0.7016

Full-matrix least-squares

4151 / 6 / 238

1.055

$\mathrm{R} 1=0.0332, \mathrm{wR} 2=0.0871$

$R 1=0.0339, w R 2=0.0876$

$0.01(4)$

n/a

0.264 and -0.154 e. $\AA^{-3}$ 


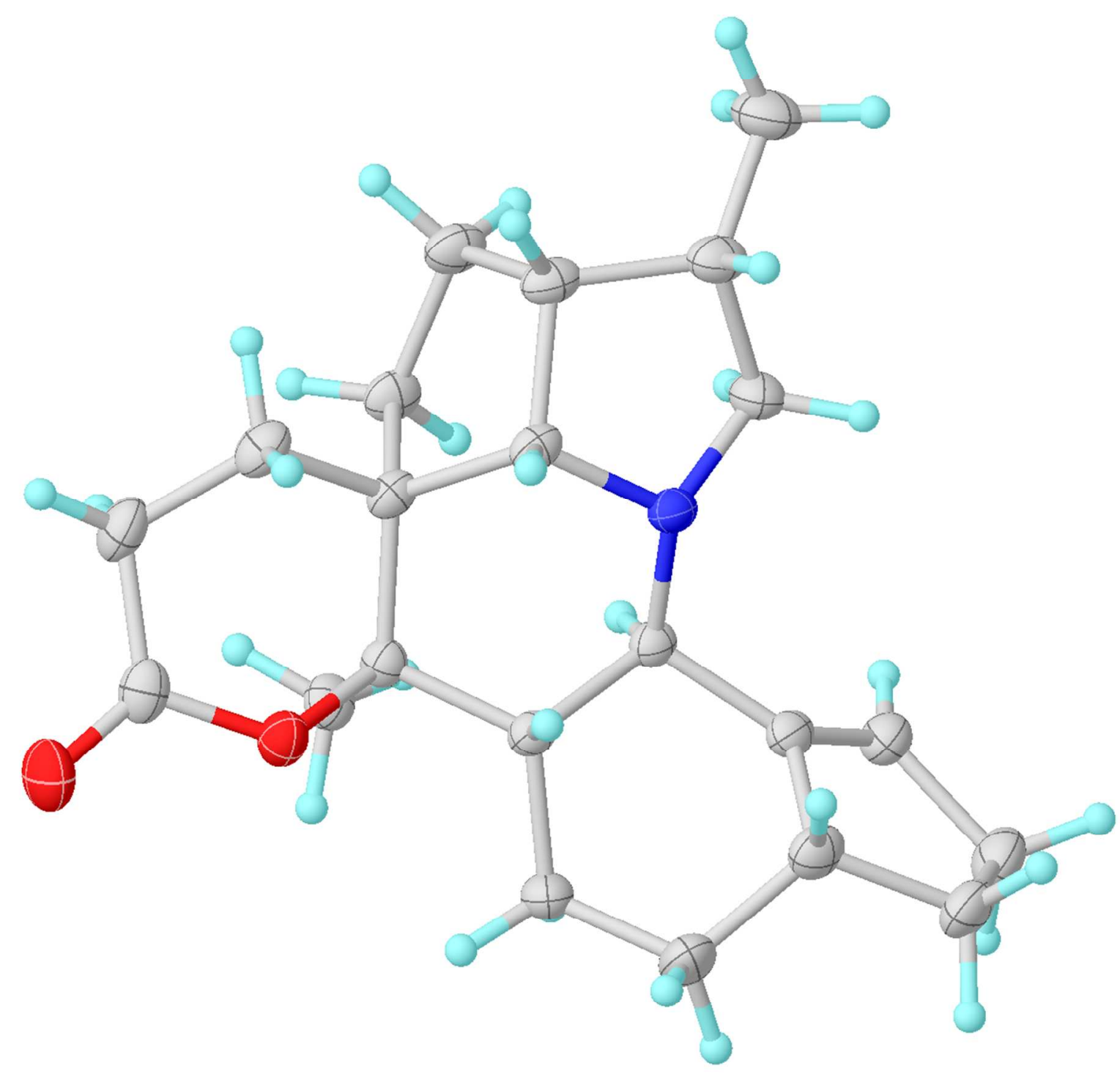

Figure S5. X-ray structure of compound 2 (CCDC1437873). Thermal ellipsoids drawn at the 50\% probability level. For DFT data, see S125. 
Table S2. Crystal data and structure refinement for $\mathbf{9}$.

$\begin{array}{ll}\text { Identification code } & \text { compound_9 } \\ \text { Empirical formula } & \mathrm{C}_{22} \mathrm{H}_{31} \mathrm{~N} \mathrm{O}_{2} \\ \text { Formula weight } & 341.48 \\ \text { Temperature } & 105 \mathrm{~K} \\ \text { Wavelength } & 1.34139 \AA \\ \text { Crystal system } & \text { Orthorhombic } \\ \text { Space group } & {\mathrm{P} 2{ }_{1} 2{ }_{1} 2_{1}} \\ \text { Unit cell dimensions } & \mathrm{a}=7.2339(2) \AA ; \alpha=90^{\circ} . \\ & \mathrm{b}=13.8782(5) \AA ; \beta=90^{\circ} . \\ & \mathrm{c}=17.7530(6) \AA ; \gamma=90^{\circ} . \\ \text { Volume } & 1782.29(10) \AA^{3} \\ \text { Z } & 4 \\ \text { Density (calculated) } & 1.273 \mathrm{Mg} / \mathrm{m}^{3} \\ \text { Absorption coefficient } & 0.401 \mathrm{~mm}^{-1} \\ \text { F(000) } & 744 \\ \text { Crystal size } & 0.24 \times 0.2 \times 0.12 \mathrm{~mm}^{3}\end{array}$

Theta range for data collection Index ranges

Reflections collected

Independent reflections

Completeness to theta $=53.594^{\circ}$

Absorption correction

equivalents

Max. and min. transmission

Refinement method

Data / restraints / parameters

Goodness-of-fit on $\mathrm{F}^{2}$

Final $R$ indices [ $>$ 2sigma( $(\mathrm{l})$ ]

$R$ indices (all data)

Absolute structure parameter

Extinction coefficient

Largest diff. peak and hole
3.517 to $60.715^{\circ}$.

$-9<=\mathrm{h}<=8,-18<=\mathrm{k}<=18,-$

$$
23<=1<=23
$$

50358

$4088[R($ int $)=0.0356]$

$99.7 \%$

Semi-empirical from

0.7519 and 0.6932

Full-matrix least-squares on $\mathrm{F}^{2}$ 4088 / 0 / 228

1.181

$\mathrm{R} 1=0.0375, \mathrm{wR} 2=0.0968$

$R 1=0.0377, w R 2=0.0970$

$0.00(4)$

$\mathrm{n} / \mathrm{a}$

0.428 and -0.435 e. $\AA^{-3}$ 


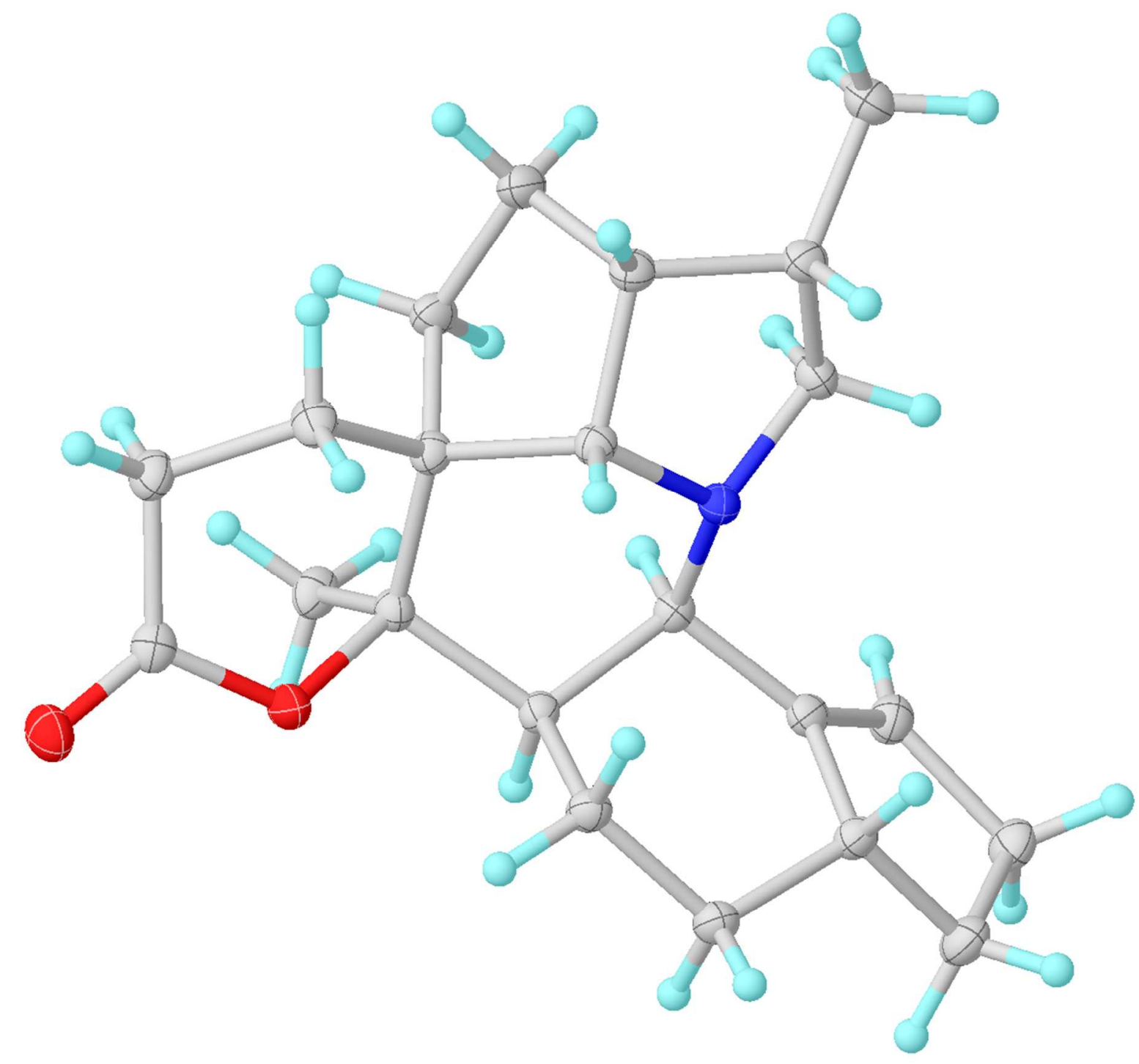

Figure S6. X-ray structure of compound 9 (CCDC1437876). Thermal ellipsoids drawn at the $50 \%$ probability level. For NMR data, see S58-59. For DFT data, see S122. 
Table S3. Crystal data and structure refinement for 25.

Identification code

Empirical formula

Formula weight

Temperature

Wavelength

Crystal system

Space group

Unit cell dimensions

Volume

Z

Density (calculated)

Absorption coefficient $\mathrm{F}(000)$

Crystal size compound_25

$\mathrm{C}_{21} \mathrm{H}_{27} \mathrm{NO}$

309.43

$110 \mathrm{~K}$

$1.34139 \AA$

Orthorhombic

P212121

$a=5.9978(4) \AA ; \alpha=90^{\circ}$.

$b=10.9505(7) \AA ; \beta=90^{\circ}$.

$c=26.0564(18) \AA ; \gamma=90^{\circ}$.

$1711.4(2) \AA^{3}$

4

$1.201 \mathrm{Mg} / \mathrm{m}^{3}$

$0.359 \mathrm{~mm}^{-1}$

672

$0.10 \times 0.06 \times 0.02 \mathrm{~mm}^{3}$
Theta range for data collection

Index ranges

Reflections collected

Independent reflections

Completeness to theta $=53.594^{\circ}$

Absorption correction

Max. and min. transmission

Refinement method

Data / restraints / parameters

Goodness-of-fit on $\mathrm{F}^{2}$

Final $R$ indices [ $1>2$ sigma $(I)]$

$R$ indices (all data)

Absolute structure parameter

Extinction coefficient

Largest diff. peak and hole
2.951 to $60.654^{\circ}$.

$-7<=\mathrm{h}<=7,-14<=\mathrm{k}<=13,-$

$33<=1<=33$

30847

$3928[R($ int $)=0.078]$

$100.0 \%$

Semi-empirical from equivalents 0.7519 and 0.5914

Full-matrix least-squares on $\mathrm{F}^{2}$ 3928 / 0 / 209

1.062

$\mathrm{R} 1=0.0430, w R 2=0.1031$

$R 1=0.0494, w R 2=0.1071$

0.04(16)

n/a

0.306 and -0.148 e. $\AA^{-3}$ 


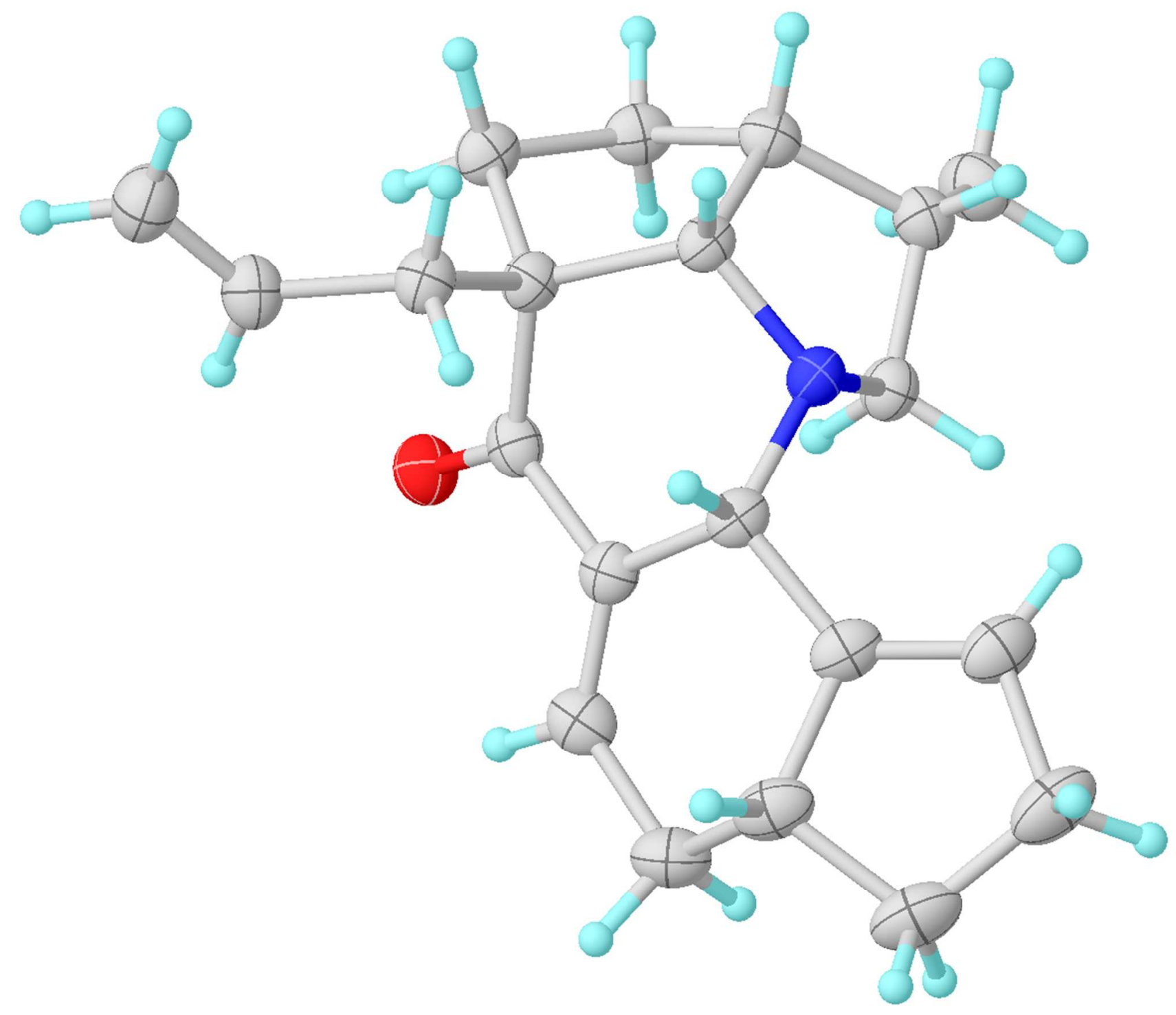

Figure S7. X-ray structure of compound 25 (CCDC1469468). Thermal ellipsoids drawn at the 50\% probability level. For NMR data, see S28-29. 
Table S4. Crystal data and structure refinement for $\mathbf{3 4 .}$

Identification code

Empirical formula

Formula weight

Temperature

Wavelength

Crystal system

Space group

Unit cell dimensions

Volume

Z

Density (calculated)

Absorption coefficient

$\mathrm{F}(000)$

Crystal size compound_34

$\mathrm{C}_{21} \mathrm{H}_{29} \mathrm{NO}$

311.45

$100 \mathrm{~K}$

$1.34139 \AA$

Orthorhombic

$\mathrm{P} 2{ }_{1} 2_{1} 2_{1}$

$a=5.5592(14) \AA ; \alpha=90^{\circ}$.

$b=11.717(3) \AA \AA$

$c=26.483(7) \AA ; \gamma=90^{\circ}$.

1725.1(8) $\AA^{3}$

4

$1.199 \mathrm{Mg} / \mathrm{m}^{3}$

$0.356 \mathrm{~mm}^{-1}$

680

$0.12 \times 0.02 \times 0.005 \mathrm{~mm}^{3}$
Theta range for data collection

Index ranges

Reflections collected

Independent reflections

Completeness to theta $=53.594^{\circ}$

Absorption correction

equivalents

Max. and min. transmission

Refinement method

Data / restraints / parameters

Goodness-of-fit on $\mathrm{F}^{2}$

Final $R$ indices [ $1>2$ sigma(I)]

$R$ indices (all data)

Absolute structure parameter

Extinction coefficient

Largest diff. peak and hole
2.903 to $57.058^{\circ}$

$-6<=\mathrm{h}<=6,-14<=\mathrm{k}<=14,-$

$32<=\mid<=33$

32676

$3525[\mathrm{R}$ (int) $=0.2757]$

$100.0 \%$

Semi-empirical from

0.7512 and 0.4789

Full-matrix least-squares on $\mathrm{F}^{2}$

3525 / 0 / 209

0.993

$\mathrm{R} 1=0.0688, w R 2=0.1306$

$R 1=0.1597, w R 2=0.1660$

$-1.1(7)$

$\mathrm{n} / \mathrm{a}$

0.215 and -0.221 e. $\AA^{-3}$ 


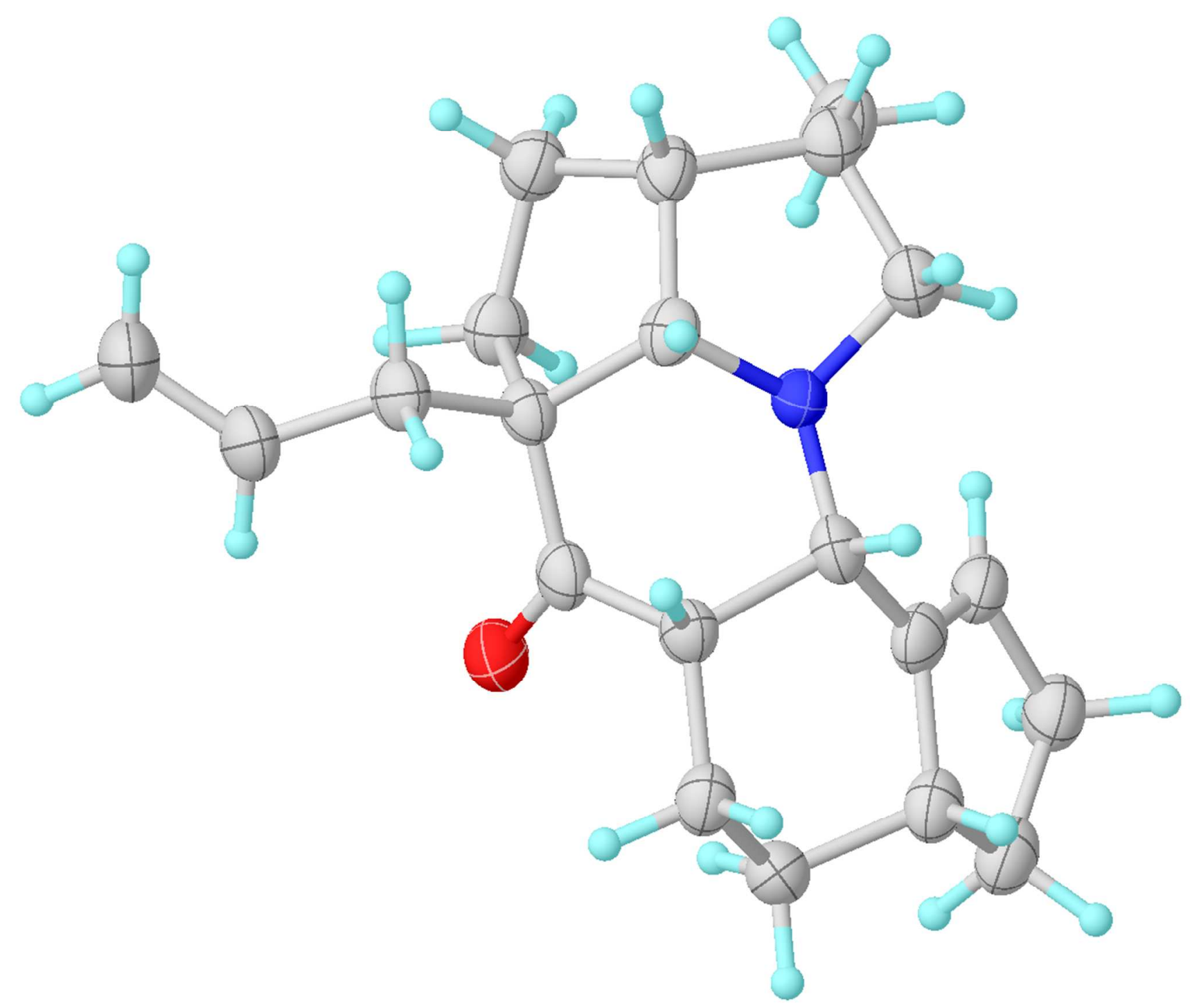

Figure S8. X-ray structure of compound 34 (CCDC1469469). Thermal ellipsoids drawn at the 50\% probability level. For NMR data, see S52-53 and S65. 
Table S5. Crystal data and structure refinement for 36_Mel.

Identification code

Empirical formula

Formula weight

Temperature

Wavelength

Crystal system

Space group

Unit cell dimensions

Volume

Z

Density (calculated)

Absorption coefficient

$\mathrm{F}(000)$

Crystal size compound_36

C23 H36 I N O

469.43

$100 \mathrm{~K}$

$1.54178 \AA$

Orthorhombic

$\mathrm{P} 2{ }_{1} 2{ }_{1} 2_{1}$

$a=10.8965(5) \AA ; \alpha=90^{\circ}$.

$b=11.6817(6) \AA ; \beta=90^{\circ}$.

$c=16.4110(8) \AA ; \gamma=90^{\circ}$.

2088.95(18) $\AA^{3}$

4

$1.493 \mathrm{Mg} / \mathrm{m}^{3}$

$12.115 \mathrm{~mm}^{-1}$

968

$0.22 \times 0.2 \times 0.06 \mathrm{~mm}^{3}$
Theta range for data collection

Index ranges

Reflections collected

Independent reflections

Completeness to theta $=67.679^{\circ}$

Absorption correction

equivalents

Max. and min. transmission

Refinement method

Data / restraints / parameters

Goodness-of-fit on F2

Final $R$ indices [ $1>2 \operatorname{sigma}(I)]$

$R$ indices (all data)

Absolute structure parameter

Extinction coefficient

Largest diff. peak and hole
4.646 to $72.207^{\circ}$.

$-13<=\mathrm{h}<=13,-14<=\mathrm{k}<=14$,

$20<=1<=20$

85832

$4122[\mathrm{R}$ (int) $=0.0466]$

$99.9 \%$

Semi-empirical from

0.7535 and 0.3436

Full-matrix least-squares on $\mathrm{F}^{2}$

4122 / 0 / 239

1.106

$\mathrm{R} 1=0.0188, w R 2=0.0505$

$R 1=0.0188, w R 2=0.0505$

$-0.003(2)$

$\mathrm{n} / \mathrm{a}$

0.443 and -0.734 e. $\AA^{-3}$ 


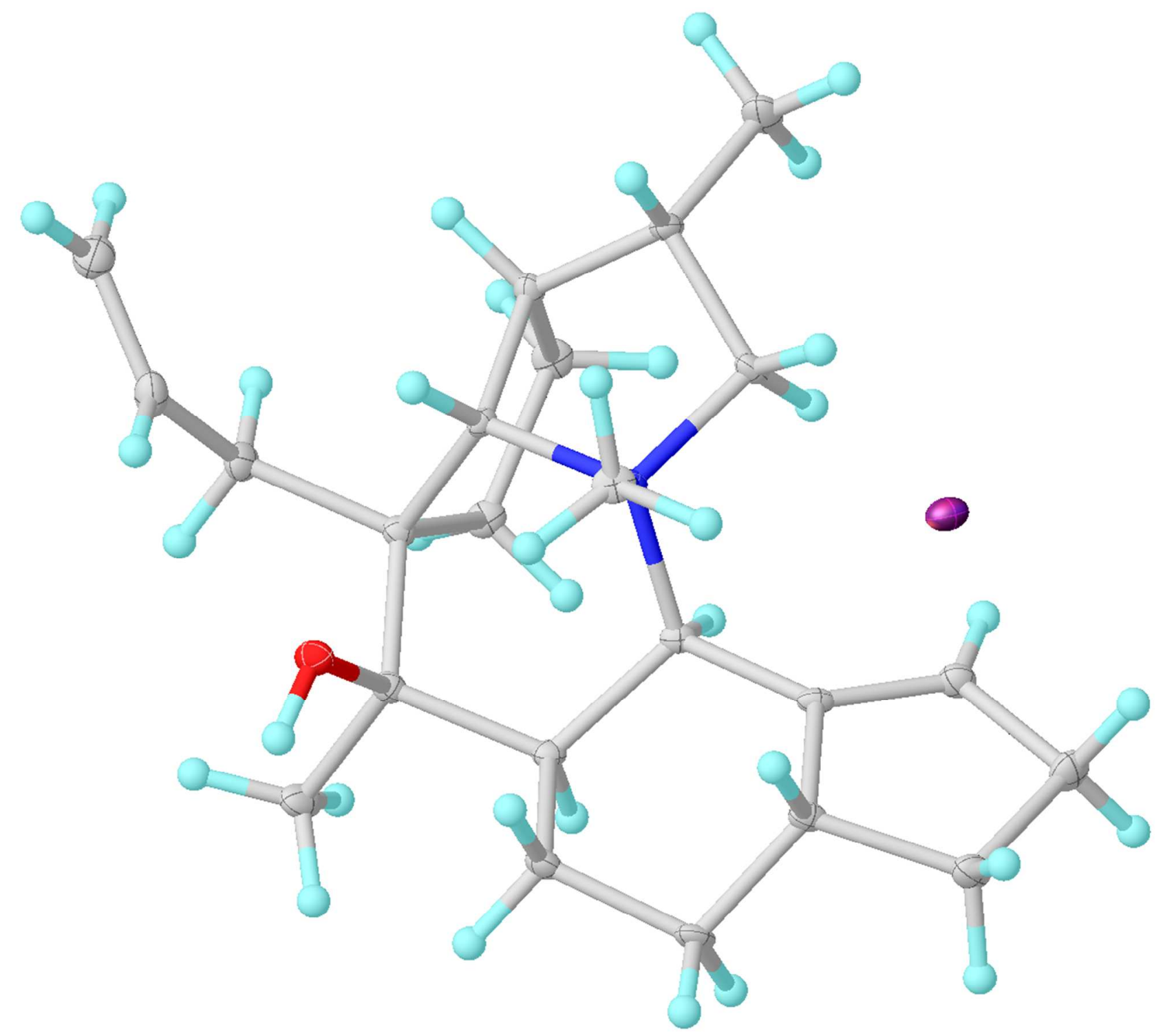

Figure S9. X-ray structure of the methiodide of compound 36 (CCDC1437875). Thermal ellipsoids drawn at the $50 \%$ probability level. 


\section{REFERENCES}

1. Chai, J.-D.; Head-Gordon, M. Phys. Chem. Chem. Phys. 2008, 10, 6615.

2. Weigend, F.; Ahlrichs, R. Phys. Chem. Chem. Phys. 2005, 7, 3297.

3. Weigend F., Phys. Chem. Chem. Phys. 2006, 8, 1057.

4. Peng, C.; Schlegel, Bernhard, H. Israel Journal of Chemistry. 1993, pp 449-454.

5. Peng, C.; Ayala, P. Y.; Schlegel, H. B.; Frisch, M. J. J. Comput. Chem. 1996, 17 (1), 49.

6. Tomasi, J.; Mennucci, B.; Cammi, R. Chem. Rev. 2005, 105, 2999.

7. Dolomanov, O.V.; Bourhis, L.J.; Gildea, R.J; Howard, J.A.K.; Puschmann, H. J. Appl. Cryst. 2009, 42, 339341.

8. Burla, M.C.; Caliandro, R.; Camalli, M.; Carrozzini, B.; Cascarano, G.L.; De Caro, L.; Giacovazzo, C.; Polidori, G.; Siliqi, D.; Spagna, R. J. Appl. cryst. 2007, 40, 609-613.

9. Sheldrick, G.M. Acta cryst. 2008, A64, 112-122. 